\title{
Organizational Identification: \\ Exploring the use of Training as an Employee Identification Marker in SMEs
}

\author{
Adetola Adekunle
}

A doctoral thesis submitted to the University of Gloucestershire in accordance with the requirements of the degree of Doctor of Philosophy in the School of Business and Technology 
Organizational identification $(\mathrm{OI})$ is a fundamental organizational behavioural concept in business that influences employee belongingness with the organization, this study identified two key categories of factors (employee and organizational) that influence the employee OI process within SME- known as OI markers.

The first category- employer situational-context markers are factors influencing employees' OI process that employees do not have implementation control over but only experience and react to, such as vertical communication. The second categoryemployee situational-gap markers however include factors such as employees' sense of in-company worth which are often cognitive and affective notions employees can control within SMEs. More specifically, emphasis was laid on ascertaining what role(s), training interventions, as a strategic human resource development (HRD) tool, play in influencing the employee OI process and thus the Ol statuses of employees in SMEs within the UK.

A conceptual OI framework was developed to facilitate the investigation using Dervin (1999) and Weick's (1993) sense-making theories. The interpretative phenomenological analysis (IPA) method of analysis was employed for this exploratory study, using data from fifteen SME employees' semi-structured interviews within the retail, health and social care, information and communication technology, financial and food industry of the UK economy.

Training interventions emerged as a dual-role OI marker, with the research outcome proposition that employee Ol statuses in SMEs occur as a vector component with both magnitude and directional attributes facilitated by employer situational-context and employee situational-gap markers. Specifically initiated and facilitated training interventions playing the roles of employer situational-context and employee situational-gap OI markers respectively.

The identified vector quality of OI statuses further enhances the body of OI literature concerned with the non-static behaviour of employees Ol status, as these can 
fluctuate between the two extremes of employee organizational disidentification or identification (EOD or EOI).

An Ol framework is proposed with which SMEs organizations could justifiably adjust their policies and procedures to imbue employees with a stronger sense of belongingness or $\mathrm{Ol}$ status within their establishments.

\section{Keywords:}

Organizational Identification, Employees, OI Markers, SMEs, Training and Sensemaking 


\section{DECLARATION}

I declare that the work in this thesis was carried out in accordance with the regulations of the University of Gloucestershire and is original except where indicated by specific reference in the text. No part of the thesis has been submitted as part of any other academic award. The thesis has not been presented to any other education institution in the United Kingdom or overseas.

Any views expressed in the thesis are those of the author and in no way represent those of the University.

Signed

Date $.7^{\text {th }}$ September, $2019 \ldots \ldots \ldots$

doi: $10.46289 /$ YHGT8523 
No amount of words is enough to express my gratitude to all who were instrumental to the completion of this work.

To my Supervisors Dr Robin Bown and Dr Philippa Ward: TO say you went beyond the call of duty to ensure I completed this work is an understatement, your encouragement, patience and always-present support helped me persevere to this end, despite the odds. I am forever in your debt.

To my Parents Pharm \& Mrs Olasupo and Olusike Adekunle: thank you for your prayers and unconditional love - this is for you both. We did it!

To my Adebola: thank you for being the best sister indeed and my pillar of strength, from which I sought solace on my wavy journey through my life's darkest tunnel.

To friends and family that cheered me on: both young and old; tall and small; and too many to name individually, thank you for the care.

To my respondents: without you this would not be possible- thank you for opening my eyes to the heights research can explore.

Finally, to my God source: my completion of this thesis proves time and time again that I am chosen, not forsaken, I am who you say I am;

You are for me, not against me, I am who you say I am;

You set me free, I'm a child of God

Yes, I am, and I know you love me! 


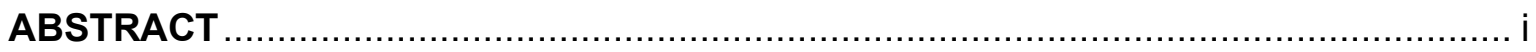

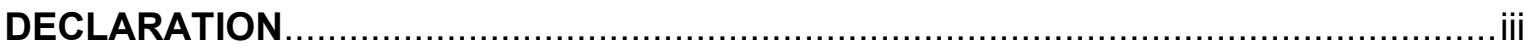

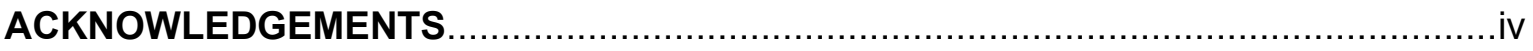

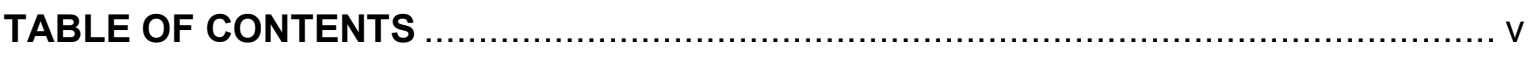

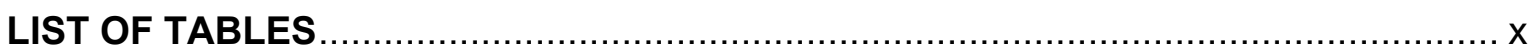

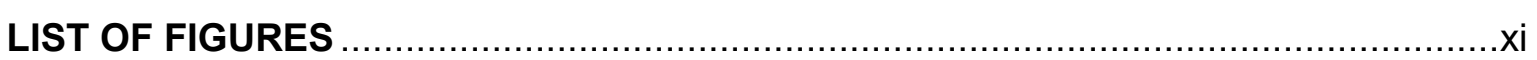

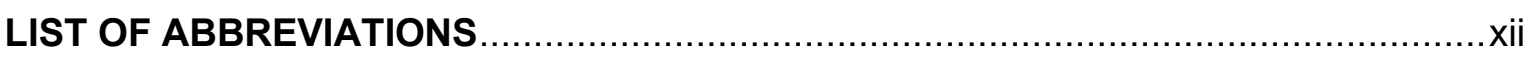

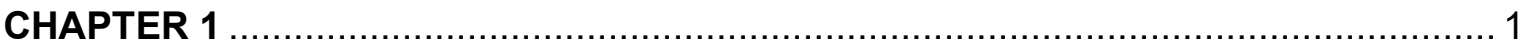

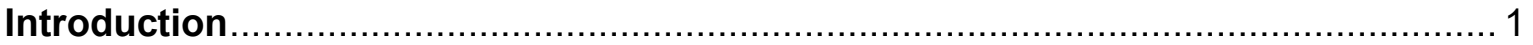

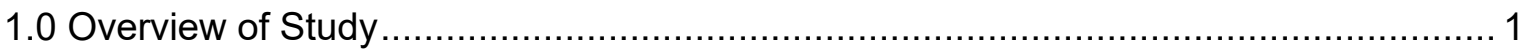

1.1 Employee Organizational Fit and Human Resource Management Strategy ............... 4

1.2 Rationale and Development of the Research Topic ............................................. 7

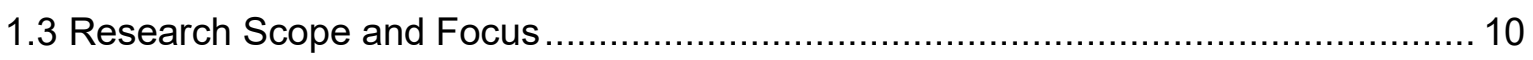

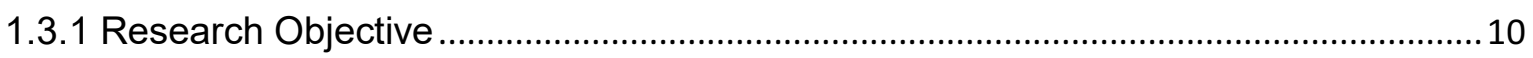

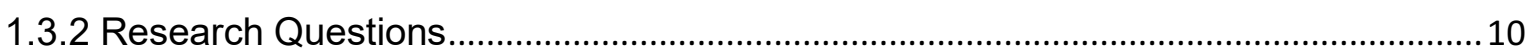

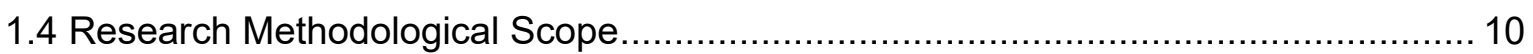

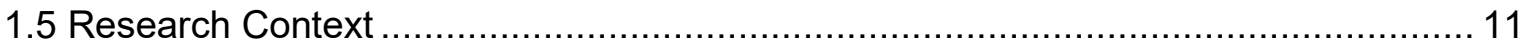

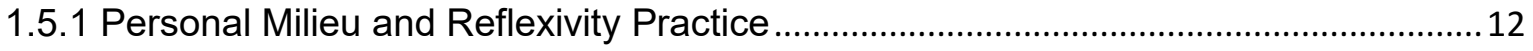

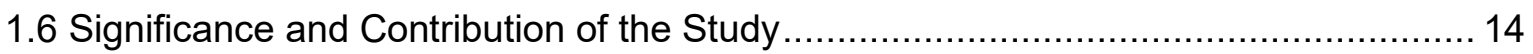

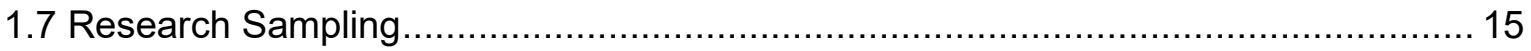

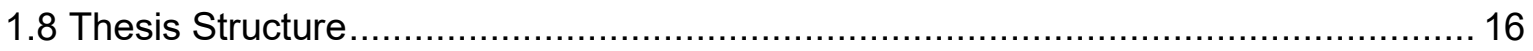

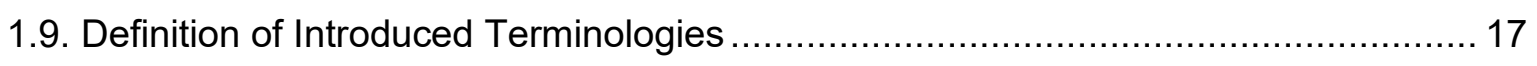

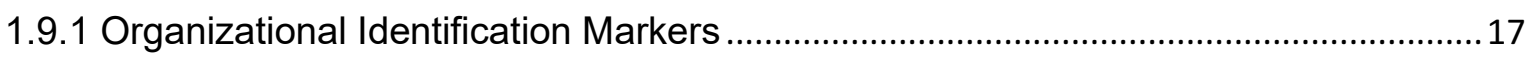

1.9.2 Employer Situational-Context OI Markers …............................................................. 17

1.9.3 Employee Situational-Gap OI Markers...................................................................... 17

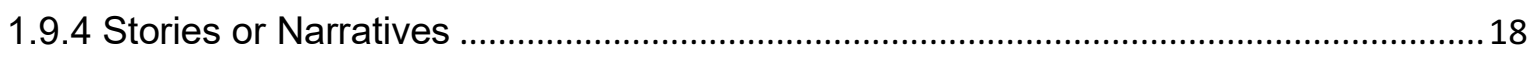

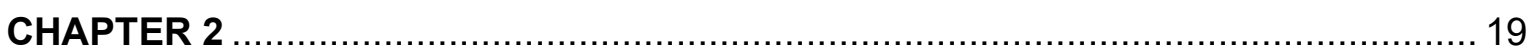

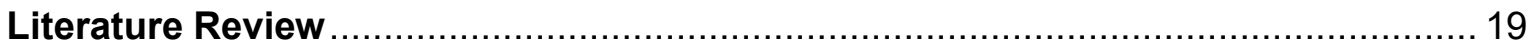

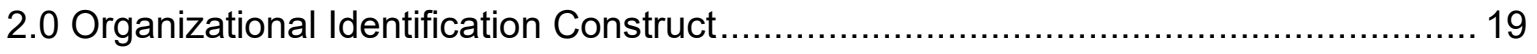

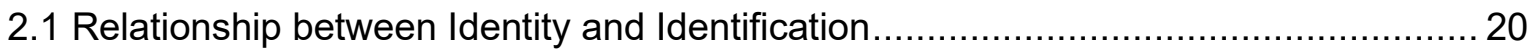

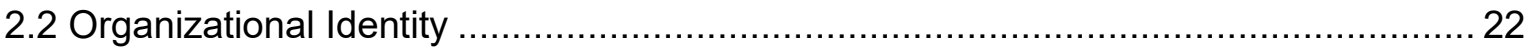

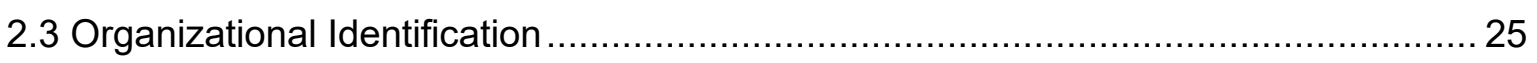

2.4 Key Organizational Identification Schools of Thought ......................................... 26

2.5 The Then and Now Evolution of Organizational Identification ................................ 29 
2.5.1 Organizational Disidentification, Ambivalent Identification and Neutral Identification

2.5.2 Organizational Identification as a Social Identity Construct........................................... 33

2.5.3 Organizational Identification as a Multi-Factorial Construct.......................................... 36

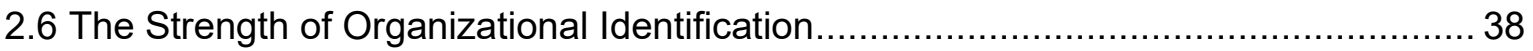

2.7 Organizational Identification Process: Control or Regulation? ................................. 39

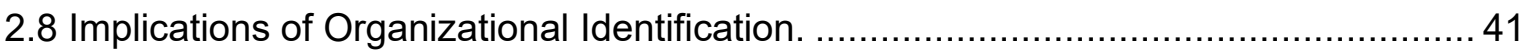

2.9 Organizational Impacts of Organizational Identification .............................................. 42

2.9.1 Organizational Identification and Organizational Commitment ..................................... 43

2.9.2 Organizational Identification, Perceived External Prestige, Organizational and Corporate Identity; and Organizational Culture ........................................................ 45

2.9.3 Organizational Identification and Communication ...................................................... 49

2.9.4 Organizational Identification, Organizational Citizenship Behaviours and Employee Unlearning

2.9.5 Organizational Identification, Power Structures, Organizational Leadership and Motivation

2.9.6 Organizational Identification, Intra-organizational Interactions and Job Satisfaction 56

2.9.7 Organizational Identification, Employee Performance Management and Organizational Practices .57

2.10 Filling the Organizational Identification Literature Gap ........................................ 59

2.10.1 Organizational Identification and Human Resource Management Practices............60

2.10.2 Understanding Employee Training in Learning Organizations...................................61

2.10.3 Small and Medium-Sized Enterprises and Business Studies ...................................68

2.10.4 Benefits of Employee Training to Small and Medium-Sized Enterprises ................. 70

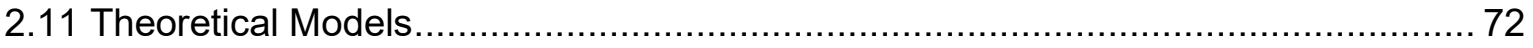

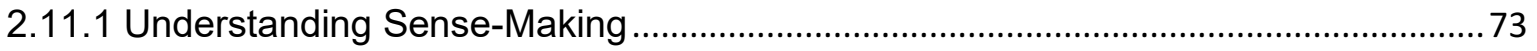

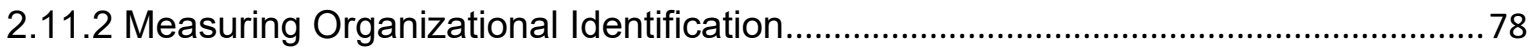

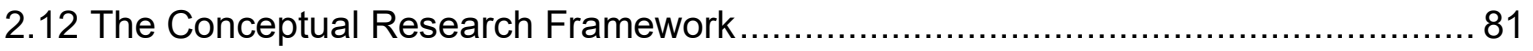

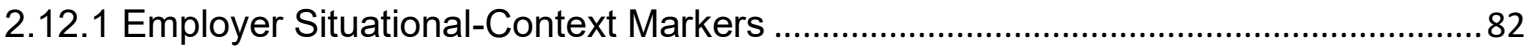

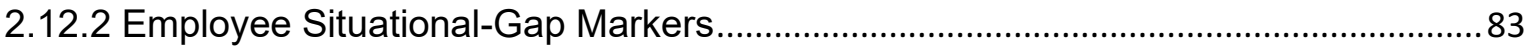

2.12.3 Employee Organizational Identification and Disidentification..................................... 83

2.12.1 Research Questions and the Conceptual Research Framework...............................83

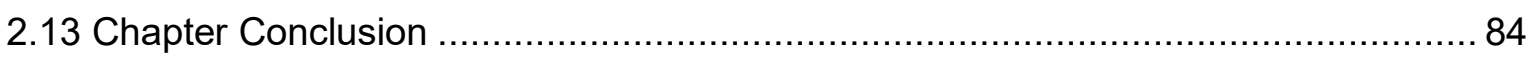

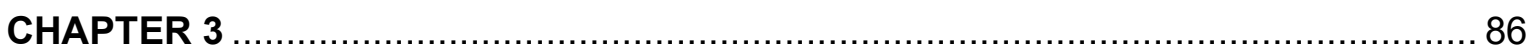

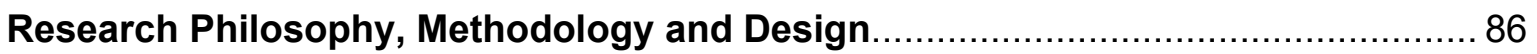

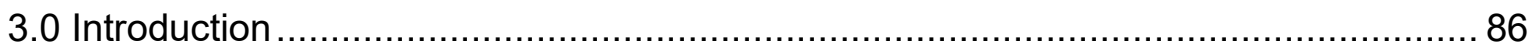

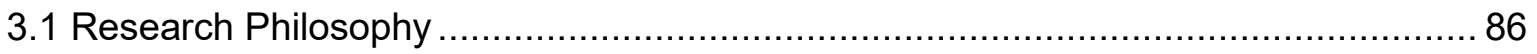

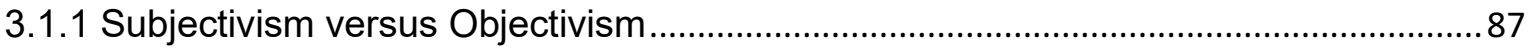




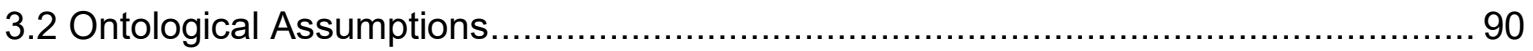

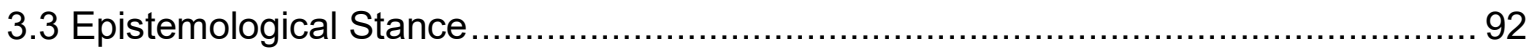

3.4 The Interpretative Phenomenological Analysis Approach..................................... 95

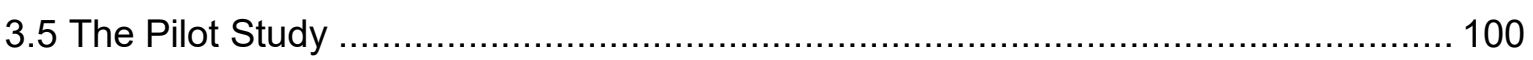

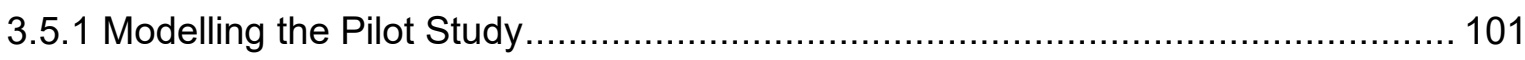

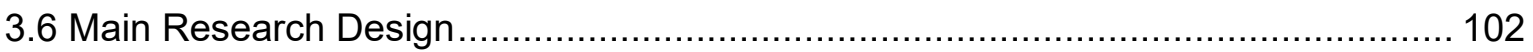

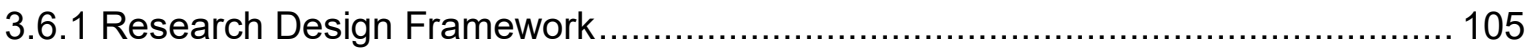

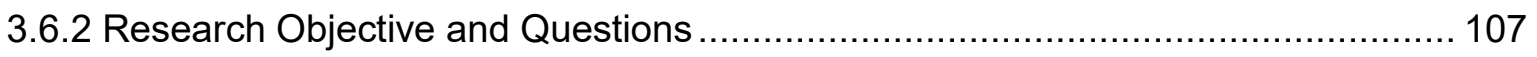

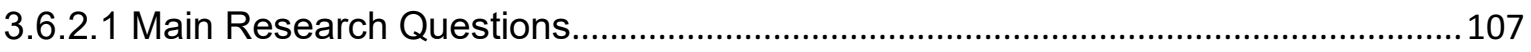

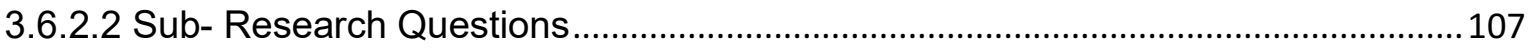

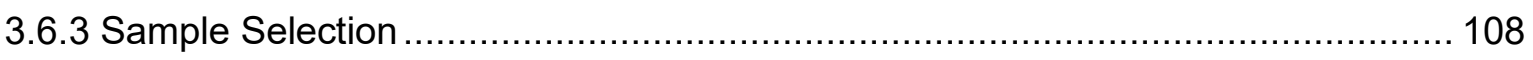

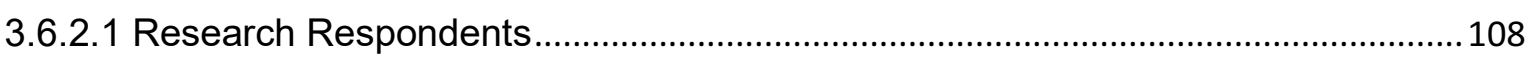

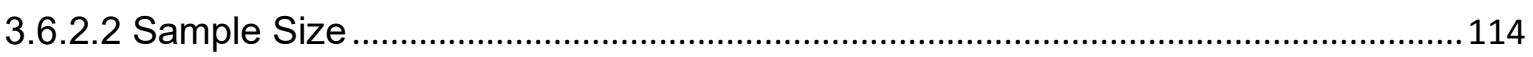

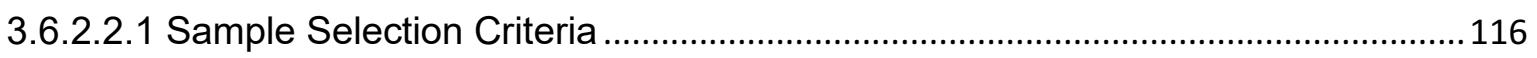

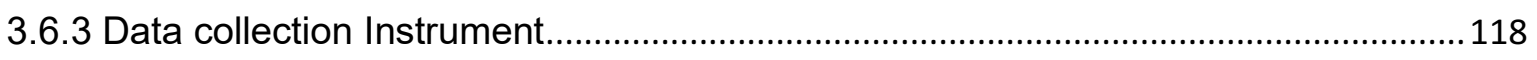

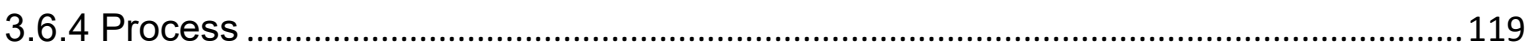

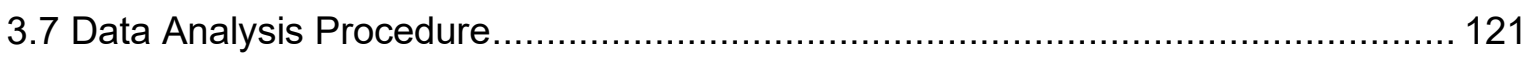

3.7.1 Quality and Methodological Rigour of Data Analysis ..............................................125

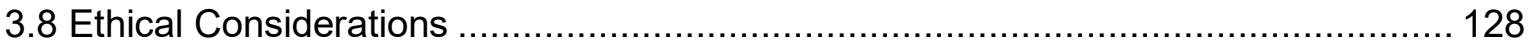

3.9 Research Governance and Organization .......................................................... 130

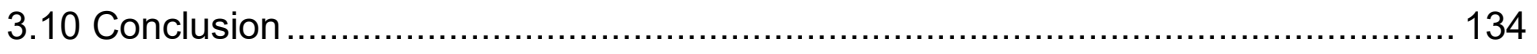

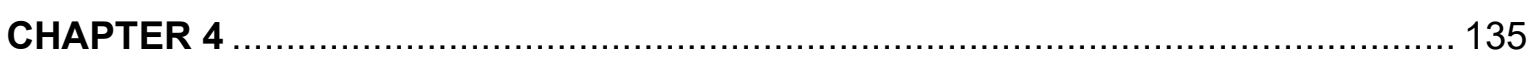

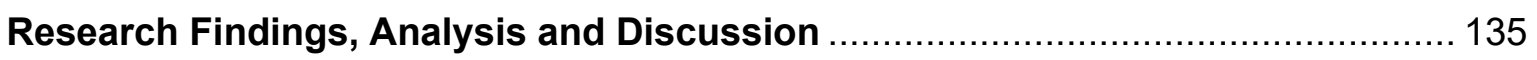

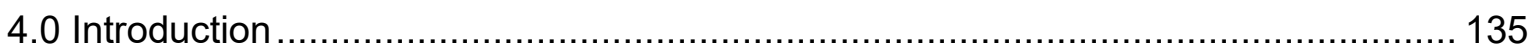

4.1 Presentation and Analysis of Pilot Study Findings............................................... 136

4.1.1 Further Pilot Analysis: Employees' Perception versus Organisational Identification

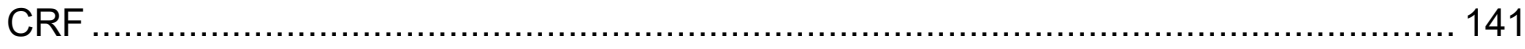

4.1.2 Force or Coercion versus Organizational Control ...................................................143

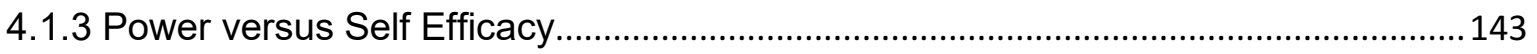

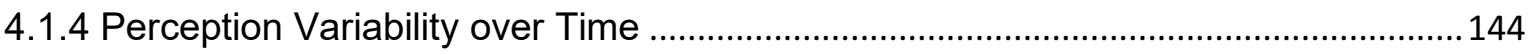

4.1.5 A Sacrificial Opportunity Cost and Perseverance ....................................................... 144

4.1.6 Receptive Attitude and Personal and Professional Fulfilment.................................... 145

4.2 Respondents in the Main Research Study ....................................................... 145

4.3 Descriptive Overview of Respondents' Stories and Narratives ............................ 147

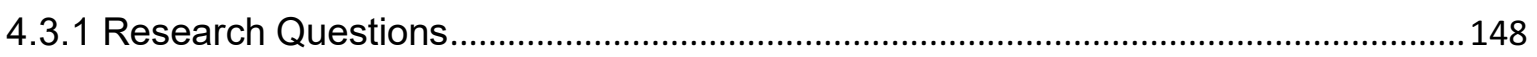

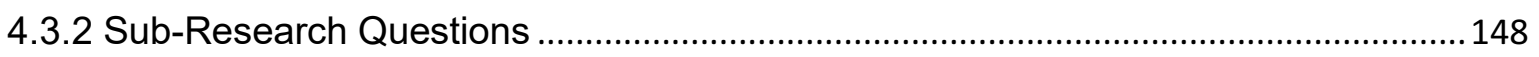

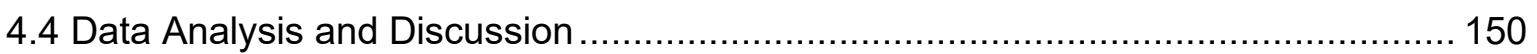




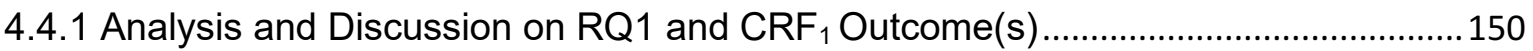

4.4.1.1 Willingness to Contribute More to the Organization ................................................. 151

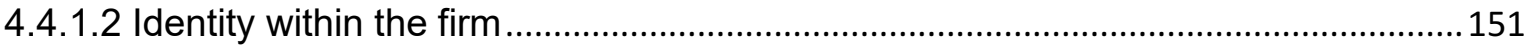

4.4.1.3 Career Aspiration Alignment with Company Objectives .......................................... 154

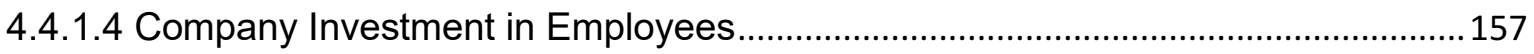

4.4.1.5 Tangible Benefits Derived from the Company ...................................................... 161

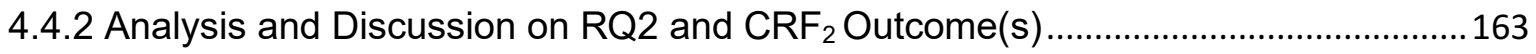

4.4.2.1 Organizational Identification: Employer Situational-Context Markers .....................165

4.4.2.1.1 Vertical Communication and Clarity of Purpose .................................................... 165

4.4.2.1.2 Ethical Management and Leadership Practices ......................................................168

4.4.2.1.3 Company Image or Identity and Culture................................................................ 175

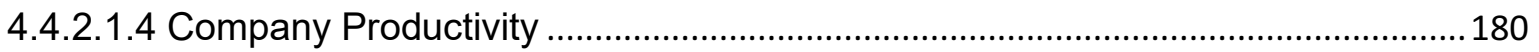

4.4.2.2 Organizational Identification: Employee Situational-Gap Markers ..........................182

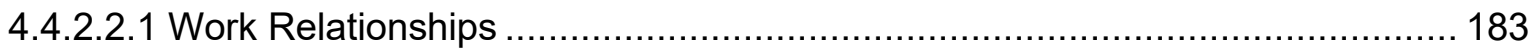

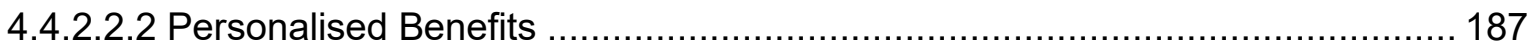

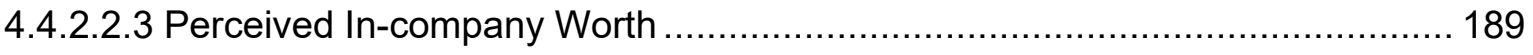

4.4.3 Analysis and Discussion on RQ3 and $\mathrm{CRF}_{3}$ Outcomes ......................................190

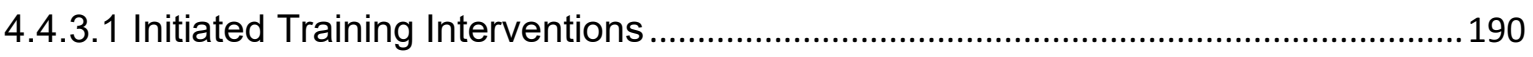

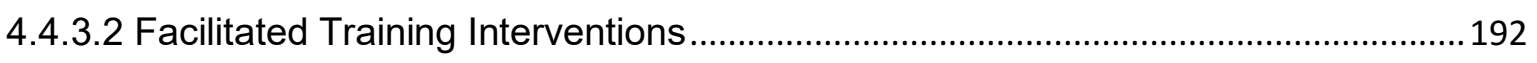

4.5 Sectoral Differences in Employee OI Outcomes................................................. 194

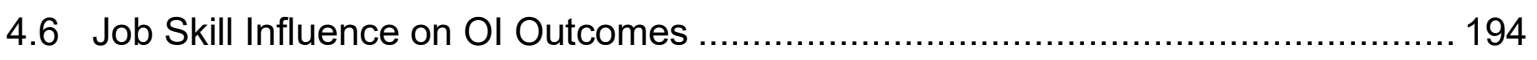

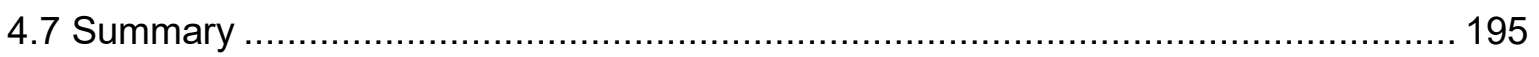

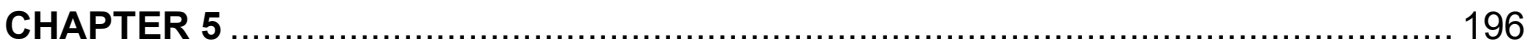

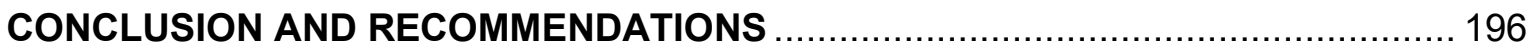

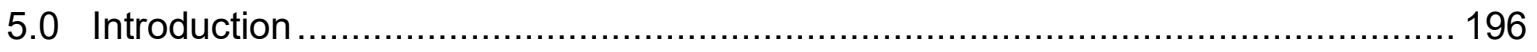

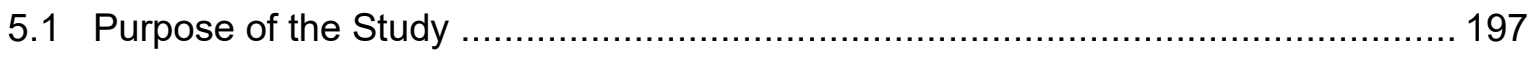

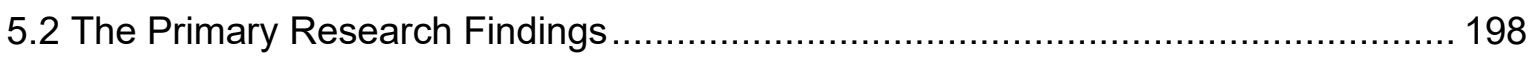

5.3 Revised Conceptual Research Framework .................................................... 202

5.4 Organizational Identification Employer Situational-context Markers ......................... 204

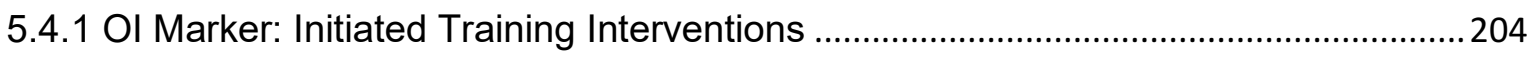

5.4.2 Ol Marker: Vertical Communication and Clarity of Organizational Purpose .............205

5.4.3 OI Marker: Ethical Management and Leadership ........................................................2. 206

5.4.4 OI Marker: Company Image/Identity and Culture ........................................................20

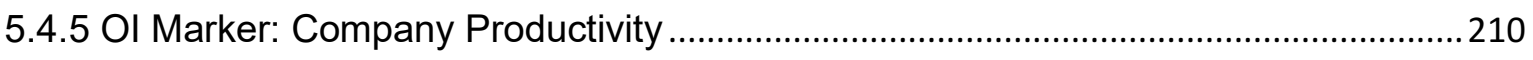

5.5 Organizational Identification Employee Situational-gap Markers ........................... 210

5.5.1 OI Marker: Facilitated Training Interventions .............................................................210 


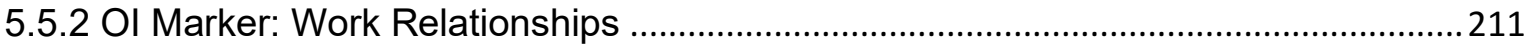

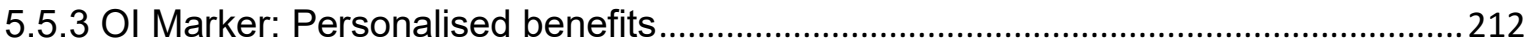

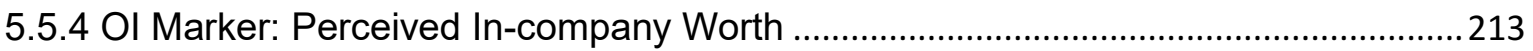

5.6 Key Research Contributions to Academic Knowledge ...................................... 213

5.7 Research Policy-Practice Implications for Small Business Organizations............... 221

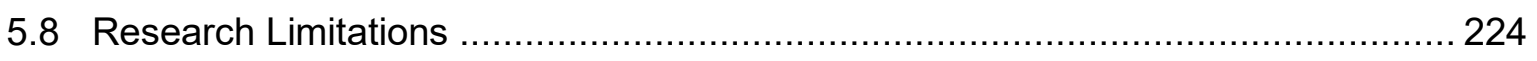

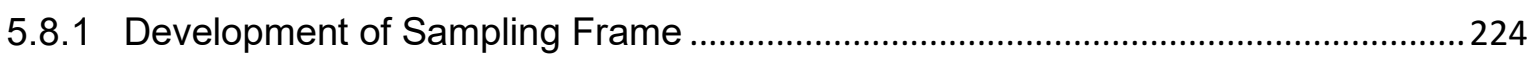

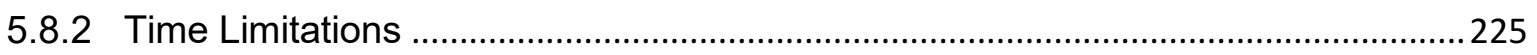

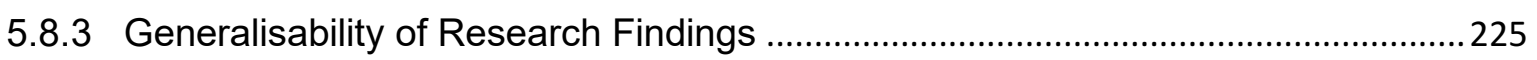

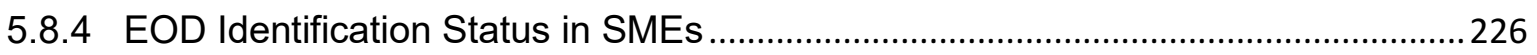

5.9 Literature Publication Emanating from the Research Thesis ............................... 227

5.10 Future Areas of Organizational Identification Research................................... 227

5.10.1 Exploration of the Owner-manager OI Perspectives on SMEs ................................227

5.10.2 Introducing a longitudinal time study perspective to the OI research context

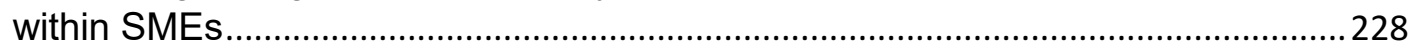

5.10.3 Exploration of the expression of Organizational Sacrifice relative to OI research in

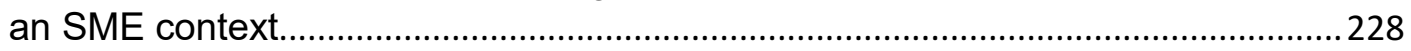

5.10.4 Exploration of the Magnitude attribute of Ol construct as a vector function in

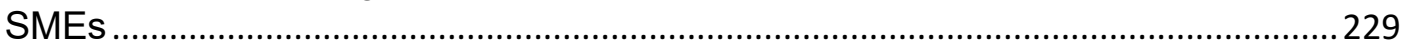

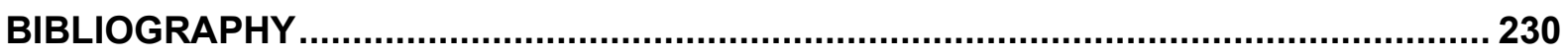

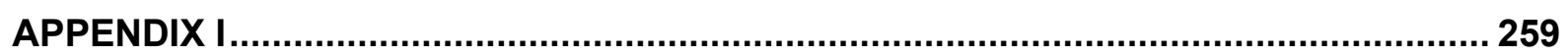




\section{LIST OF TABLES}

Table 1 - Demographic Distribution of the Pilot Study Respondents. .................... 102

Table 2 - Demographic Distribution of Research Respondents ........................... 117

Table 3 - Comparison of highlighted employee OI markers in SMEs, pre- and postresearch interview sessions 


\section{LIST OF FIGURES}

Figure 1 - Diagrammatic Representation of Weick's Sense-making Theory adapted from Weick (1993)

Figure 2 - Diagrammatic Representation of Dervin's Sense-making Theory adapted from Snowden (2008)... 76

Figure 3 - Podnar, Golob \& Jancic, (2011) OI Measuring Scale. 80

Figure 4 - Organizational Identification Conceptual Research Framework. 82

Figure 5 - Sub-sections within the Conceptual Research Framework 84

Figure 7 - Research Design Framework 106

Figure 6 - Pilot Study Emergent Themes 142

Figure 8 - Descriptive Overview of Respondents Stories and Narratives. 149

Figure 9 - Revised Conceptual Organizational Identification Research Framework 203 


\section{LIST OF ABBREVIATIONS}

CRF

DBEIS

DBIS

EL

EOD

EOI

EU

HRM/D

IPA

MA

OB

OI

PEP

PGJ

POF

RDF

RQ

SME

SRQ
Conceptual Research Framework

Department for Business, Energy, Innovation and Strategy

Department for Business Innovation and Skills

Ethical Leadership

Employee Organizational Disidentification

Employee Organizational Identification

European Union

Human Resource Management and Development

Interpretative Phenomenological Analysis

Mael and Ashforth-OI Scale

Organizational Behaviour

Organizational Identification

Perceived External Prestige

Podnar, Golob and Jancic-OI Scale

Parent/Older-Sibling Figure

Research Design Framework

Research Question

Small and Medium-sized Enterprises

Sub-Research Question 


\section{CHAPTER 1}

\section{Introduction}

"...I am one of those who think like Nobel, that humanity will draw more good than evil from new discoveries...\&... Find out what's really out there. I never said to be like me, I say be like you and make a difference..." (Marie Curie \& Marilyn Mason)

\subsection{Overview of Study}

In contemporary commerce, the dynamics of business operations are constantly changing. Many changes are often associated with the influence that the use of information and communication technology exerts on organizational activities. Despite the ever-evolving nature of the business environment, however, employees are still considered to be at the heart of most businesses' attempts to compete effectively in the market.

The importance of employees is often considered to be particularly true for small businesses, who due to limitations - such as limited funding, staff numbers, as well as geographical coverage relative to their larger businesses counterpart - are often at a disadvantage (Lee \& McGuiggan, 2008; Valadez, 2011). Hence, most small businesses' competitive advantage indices are a mirror reflection of the skills and expertise of their employees, rather than any other resource within the organizations (Valadez, 2011; Oriaku, 2012)

Furthermore, statistical evidence from business population estimates in 2017, by the Department for Business, Energy and Industrial strategy (DBEIS, formerly known as the Department for Business Innovation and Skills) shows that small businesses account for over ninety-nine percent of the overall population of employees in every main industry sector in the United Kingdom economy. The researcher's first-hand experience of working as an employee in several small businesses across two continents also resonates with the notion that employees often play a major role in the success (or non-success) stories of such businesses. This contributes 
significantly to the choice of situating this research within the small and mediumsized enterprises (SMEs) context.

This study aimed at broadening and adding value to the existing knowledge and literature on the psychological concept of employee organizational identification (EOI) in SMEs, as organizational identification is generally understood to be a strong determinant of employees' belongingness and commitment to an organization (Boros, 2008; Riketta, 2005). This research focused on employees' stories and from these stories an interpretation of their perceived understanding of organizational identification is developed. The study further explored the concept of organizational identification by identifying the factors, referred to as markers in this study, which facilitate the process and formation of employees' OI status in SMEs within the UK.

The study in particular explored the use of training interventions as a human resource developmental tool, based on the narrated experiences of the SME employees. It sought to investigate how (if possible) organizations could tailor their training activities to develop employees' organizational identification, rather than foster, or fail to dispel, organizational dis-identification, the development of the former and the minimisation of the latter are crucial to achieving organizational goals (Mael \& Ashforth,1989).

To facilitate this exploration, a conceptual framework was designed that drew on the sense-making theories of Dervin (1999) and Weick(1993), as well as the organizational identification measuring scales of Mael and Ashforth (1992) and that of Podnar, Golob and Jancic (2011). The framework proposed strived to identify the factors impacting the formation of either EOI or dis-identification (EOD).

Having employees with EOI in companies often translates to alignment of an employee's personal objectives to those of the organization. This is in contrast to EOD, whose effect, if developed by employees, may be detrimental to obtaining organizational objectives. Both statuses (EOI and EOD) have variants that are detailed in various organizational identification related literatures (see for instance: Dukerich, Kramer \& Parks,1998; Elsbach \& Bhattacharya, 2001; Kreiner \& Ashforth, 2004; Dukerich, Kramer \& Parks, 1998; Boros, 2008; Podnar, Golob \& Jancic, 2011), and whilst EOI and EOD may appear binary, each is composed of a variety of 
statuses that when considered collectively can be broadly seen as demonstrating alignment (EOI ) or separation (EOD) of the employee and the organization.

The study also explored the relevance of the group identification component introduced by Ol literatures, such as the work of Podnar, Golob and Jancic (2011), and its impact on the employee $\mathrm{OI}$ process and formation. This consideration served to add to the interpretation of the Ol construct from an individualistic lens.

Situated with the SME context, the study data collated was from participants working across various economic sectors of the UK, such as the retail, health and social care, information and communication technology, financial, as well as food industry domains. One of the rationales for the choice of participants from these sectors is the furthering of the academic literature on OI within these sectors, such as that of Kim, Chang and Jae-Ko (2010); Farrell and Oczkowski (2012) and Tsui and Ngo (2015), and to capture the significance of training interventions in these sectors.

Another rationale rests on the reliance of these sectors on their workforces and it could be suggested that given this, employees are also anchors to the successes of their companies. Such successes include improved sales and productivity, better customer satisfaction figures and overall growth of the businesses (Lee \& McGuiggan, 2008; Valadez, 2011; Ferdous \& Polonsky, 2014). However, prior to this research, there was still a significant paucity of literature across the sectors researched here in comparison to more popularly considered areas such as engineering and the health sciences (Carlin, End \& Mullins, 2010; Bacaksiz, Tuna \& Seren, 2017).

In addition to the reasons above, the economic significance of the sectors examined cannot be overlooked. The retail service industry alone contributes a dominant percentage share of approximately $34 \%$ to the UK SMEs' financial turnover ratio; information and communication technology about 6\%; the food industry and health and social care services contributing $3.5 \%$ on average each. Cumulatively, the researched industries contribute about $50 \%$ of total UK SME turnover (DBEIS, 2017). With SMEs accounting for at least $99.5 \%$ of the businesses in every main industrial sector of the nation, these sectors' importance to the economic development of the nation cannot be overstated (DBEIS, 2017). 
The literature reviewed also highlighted the relationship that exists between organizational identification and other organizational concepts. Such concepts include organizational commitment; job satisfaction; employee performance and leadership (Mael \& Ashforth, 1992; Carmeli \& Freund, 2002; Boros \& Curseu, 2005; He \& Brown, 2013; Riketta, 2005; van Knippenberg \& Sleebos, 2006; Boros, 2008). Others include perceived external prestige; organizational citizenship behaviour; leadership; favourable work experiences; job crafting/employee creativity; employee turnover; communication (Freund, 2006; Meyer, Allen \& Smith., 1993; Branscombe \& Wann, 1994; Brett, Cron \& Slocum 1995; Becker et al., 1996; Meyer and Allen, 1997; Ellemers et al., 1999; Bergami \& Bagozzi, 2000; Podsakoff et al. 2000; Ciftcioglu, 2010); and corporate social responsibility (Humphrey, 2012; Peters et al., 2012). As such, $\mathrm{OI}$ could be seen as arguably connected to a wide range of other organizational concerns that are seen to be instrumental to the success and continued development of businesses.

\subsection{Employee Organizational Fit and Human Resource Management Strategy}

Emphasising the significance of employees in organizations, as iterated in the previous section 1.0, Muller-Carmen, Croucher and Leigh (2008) highlighted their shared belief in a people management strategy as an important tool in generating favourable organizational outcomes. This people management strategy is synonymous with what is more commonly known as 'human resource management' (HRM), which is a framework or methods organizations use to manage their people resources, in a way that supports long-term business goals and outcomes (CIPD, 2019). That is, HRM constitutes an organization's way of making efficient use of its existing human resource (employees) to achieve its organizational objectives.

According to Muller-Carmen, Croucher and Leigh (2008), the people management strategy philosophy, which essentially hinges on the good treatment of employees principle to produce better organizational results, arose as a converging solution to the general notion that only HR practitioners essentially value and respect human resources in comparison to other designated line managers in organizations. In addition, the principle stresses the importance of choices made by organizational management to own and believe in the decisions made - for instance in relation to what kind of training is done, the pay system employed, as well as the kind of 
people-friendly policies created within the business (Gratton et al., 1999; MullerCarmen, Croucher \& Leigh, 2008). At the heart of this people management strategy is the notion that human resources within a business essentially contribute to the maintenance of the core competencies of the business. This is particularly evident in terms of expertise generation, wherein skilled individuals are constantly being recruited and trained, as well as providing the crucial competitive advantage for the organizations in which they work (Mayo, 2008).

Thus, this opinion above closely links to the historical evolution of what is now contemporarily known as the professional practice of human resource management and development (HRM/D), embraced by practitioners and academics alike since the 1980s and originating from the UK and United States(US) (Wilson, 2005; Collings \& Wood, 2009). This is also in agreement with the more recent strategic practice perspective within HRM of Becker and Huselid (2006), which could be suggested to be an essential element of management practice directly linked to the improvement of the overall level of organizational performance. That is, it is imperative for organizations to strategically align their HRM/D practices with their organizational goals and objectives to gain improved organizational outcomes.

Furthermore, in support of the people management strategy, Boydell (1971), in his work on identification of training needs as an HRM/D tool, asserted that training interventions could rationally occur in three key areas within the organization, namely individual, occupational and organizational development. According to Boydell, individual development is usually broad and addresses people management areas in the workplace such as skill development, interpersonal skills and career development.

Occupational development, on the other hand, emphasises the need to integrate cross-functional workers, through group building related activities. Organizational development, however, focuses on the entire organization and according to Robbins (1993) is defined as "A collection of planned change interventions, built on humanistic-democratic values, that seek to improve organizational effectiveness and employee- well-being." That is, organizational development provides viable ways and options to improve organizational effectiveness and employee happiness through planning, organising and implementing employee developmental activities. Thus, 
such activities or interventions, which are often HRD interventions like training, if aptly implemented can contribute wholesomely to the well-being and effectiveness of the organizational workforce.

Noe (1999) defined training as "a planned effort by a company to facilitate employees' learning of job-related competencies"; similarly, Roy and Raymond's (2008) definition as "a medium for developing human resources in business establishments, to motivate employees and improve their on the job performance" has been linked to job satisfaction, which is an element of employee well-being.

In their 2008 study on training effectiveness relative to employee attitudes in the workplace, Sahinidis and Bouris (2008) found that employees derive motivation and job satisfaction from training. Thus, it is observed that training has a positive impact on the daily activities undertaken by employees in organizations. Therefore, ensuring the satisfaction of employees in their respective job roles, through training activities employed within organizations, is an important duty or role business organizations need to constantly ensure they implement or improve on.

A criticism of HRM/D professional practice is, however, raised with questions around the credibility of HRM/D practice tools, such as training, in contributing to general organizational success. That is, is there a quantifiable effect of these HRM/D tools in improving organizational performance? Inquiry in this respect has given rise to two varying schools of thought, namely the universalistic and contingent approaches of HRM (Delery \& Doty 1996; Becker \& Huselid, 1998).

The universalistic approach of "best practices" stresses the significance of certain HRM practices, such as training, evidentially outperforming other HRM related practices in improving organizational performance; while the contingent approach of "best fit" advocates for context dependent HRM practices. Thus, encouraging the strategic fit of varying HRM practices deployed within organizations (Huselid,1995; Becker \& Huselid, 1998).

Gratton et al. (1999), using a content approach, discerned levels of this strategic fit 
varied from offering a weak to a strong link. In this same study, they measured this strategic fit as the alignment between the set of strategic objectives and people policies that in turn create and support the individual behaviour and the competences that have the potential of becoming a source of competitive advantage for the organization. Consequently, while a strong link between individual objectives and business goals implies that the business objectives are translated into clear individual objectives, a weak linkage, on the other hand, means no clear mechanism of communicating business strategy to individual employees. That is, weak links result in situations where there are no means through which individual tasks and behaviours are discussed. This could then result in circumstances where employees do not identify with the organizational objectives set (Gratton et al.,1999).

\subsection{Rationale and Development of the Research Topic}

Studies on identities at the organizational level started gathering much interest in the 1980s after Albert and Whetten's (1985) work on identity and identification studies. They highlighted the aspects of collective (group) and selves (individual) and their implications on organizational identity and identification in relation to practice.

Organizational identification has since been developed and understood to be impactful in understanding a range of organizational strategies such as change management (Ravasi \& Phillips, 2011), decision-making (Riantoputra, 2010), internal conflicts (Humphreys \& Brown, 2002), communication (Fombrun, 1996), issue interpretation and response (Dutton \& Dukerich, 1991; Gioia \& Thomas, 1996), and as also salient in the theorisation of legitimacy issues (He \& Baruch, 2010; Sillince \& Brown, 2009).

Thus, from the employee organizational fit discussion presented above, it can be argued that appropriately deploying training as a strategic fit for business objectives and individual objectives, as opined by Gratton et al. (1999), could be key to managing a workforce with EOI statuses in organizations. That is, training as an HRM/D tool in organizations can act as a catalyst in ensuring employees' ownselfobjectives strategically fit with set business objectives.

This assertion is also supported by Hekman et al.'s (2009) argument that 
organizational identification improves employees' performance quality on the job, such as reducing their resistance to information technology. Furthermore, Albert, Ashforth and Dutton (2000), explained that organizational identity and organizational identification processes that are "root constructs in organizational phenomena" essentially form the bedrock on which many observable employee organizational behaviours crucial to every organization are built. In agreement with this notion, $\mathrm{He}$ and Brown (2013), in their literature review on the subject of organizational identity and organizational identification, also stressed that the last two decades have witnessed the significant use of organizational identity and organizational identification constructs as theoretical lenses, informing organization and management research. Thus, these ideas should occupy centre stage positions in research efforts to better understand organizations and their interactions with their environment.

He and Brown (2013) furthermore mentioned the possible relationship that exists between organizational identification and global issues, such as climate change, economic crisis and public spending cuts, making $\mathrm{Ol}$ a topical issue of interest with considerable room to enhance the scope of previous research literature on the subject. That is, essentially these linkages relate to the possibility of employees' OI status within an organization determining their performance levels relative to how they reason, implement or act on these topical issues. For example, employees' OI status (which is their level of belongingness to their organization) may determine whether or not they continue to work positively with the organization to achieve its objectives irrespective of funding setbacks due to public sector cuts.

Both researchers further highlighted that such expansion of Ol study scope could include: mapping the relationship between the different approaches to Ol study by various scholars that actively try to understand and explain the behaviour of individuals, groups and organizations; secondly, understanding the role of $\mathrm{Ol}$ in motivating employees to engage in both organizational in-role and extra-role behaviours and performance. Other plausible research areas could include study of the influence of leadership on $\mathrm{OI}$ and hence employee performance; the relationship between $\mathrm{Ol}$ and change management; and also, how $\mathrm{Ol}$ is developed within an organization. 
The paucity of literature on addressing some of the possible areas of further study about OI was further highlighted by Peters et al. in their 2012 organizational identification and strategy study, stressing that there are still significant areas of OI research that have not been explored and given the evidential assertion that $\mathrm{OI}$ underpins a range of salient organizational outcomes, it is an important area of management research, that needs to be further explored; especially in relation to how organizations that aspire to develop Ol among their employees can accomplish this.

Thus, following from this knowledge, Peters et al. (2012) used the ASPIRe (Actualizing Social and Personal Identity Resources) model (Eggins et al., 2008; Fiol, Pratt, \& O'Connor, 2009; Jans, Postmes, \& Van der Zee, 2011) to test the feasibility of building $\mathrm{Ol}$ among senior military health services personnel via bespoke workshop activities structured to use subgroup identities as a basis for OI development within the employees.

The results of their study were mostly in alignment with the predicted outcome, such as participants reporting an increased level OI due to the workshop undertaken and the participants consequently being more supportive of the organization's strategy. The workshop intervention had been a developmental tool and was the main instrument for determining the $\mathrm{OI}$ of the research participants. One of the limitations evident from the Peters et al. (2012) study was, however, the near homogeneity of research participants in relation to demographic characteristics such as gender (mostly men), race and their designated roles/position within the organization.

Thus, expanding on Peters et al. (2012) recommendations, the main purpose of this thesis was to explore how training as a strategic human resource management tool can influence the employee Ol process within SMEs. As a human resource management tool, training has been seen to substantially contribute to the improvement of overall employee job satisfaction, motivation, as well as improved organizational performance (Becker \& Huselid, 1998; Sahinidis \& Bouris, 2008). Hence, training can potentially be used as a strategic tool with the viable possibility to influence the determinants of the employee Ol process (Peters et al., 2012). 
Also, furthering He and Brown's Ol literature review studies in 2013 and Podnar, Golob and Jancic's (2011) work, this research specifically explored the possibility of identifying OI markers or markers that impact employees' OI processes, with a particular interest in examining how organization training interventions could help develop employees' EOI statuses.

\subsection{Research Scope and Focus}

This study aimed at exploring the concept of organizational identification within small businesses (also called SMEs) by identifying the underlying determinants of employees' Ol statuses within the small business context. It particularly considered and explored how training, as a strategic HRD tool, could potentially influence individual employees' OI processes.

\subsubsection{Research Objective}

The main research objective was to explore the use of training interventions as an employee organizational identification maker within SMEs. To address this primary objective, three research questions were developed:

\subsubsection{Research Questions}

1) What does employee organizational identification mean within SMEs?

2) What are the underlying markers of employee organizational identification in SMEs?

3) How do training interventions influence the employee identification process in SMEs?

\subsection{Research Methodological Scope}

As organizational identification is construed as a psychological construct within the extant literature, the narrated stories and experiences of employees in small businesses were the primary data generated and analysed within the research. This is in the form of textual data and thus aligned with a qualitative methodological approach, which defines and elucidates a contextual understanding of behaviour, values, and beliefs of the people under study (Murphy et al., 1998).

Also, in support of the qualitative methodology chosen, I wanted to understand and 
interpret the perceptions of the research participants, which aligned with the rationale provided by Bryman and Bell (2003) that a qualitative methodology provides quality data due to the subjective involvement of individuals under study in their natural environment. Thus, a researcher interacts with the primary actors to gain better understanding of the subject matter (Denzin \& Lincoln, 2003, p. 13).

The interpretation of the participants' perceptions on the research topic is framed by my previous organizational experiences. This is related to the assertion by Smith, Flowers and Larkin (2009, p. 57) that qualitative research interviews are essentially "a conversation with a purpose" and here that purpose is negotiated between the researcher (and my experiences) and the research participants (and their experiences). As such, the Interpretative Phenomenological Analysis (IPA) method was employed in data generation and interpretation within this ideographically focused research.

Another rationale for the application of IPA is the ability of the method to facilitate an in-depth understanding of lived experiences, framing the played-out perceptions of the employees under study (Singh, 2016) relative to the subject matter under discussion. Also, though widely used in psychological studies of lived experiences of research subjects (Smith, Flowers \& Larkin, 2009), IPA is now increasingly being employed as a newer methodology within qualitative research studies in business and management.

\subsection{Research Context}

The choice of small businesses as the research context was primarily because despite the perceived importance of employee organizational identification to any organization, the focus of much academic literature has been on multinational companies (MNCs). The rationale for this prior literature focus on large businesses, as suggested by Valadez (2011), may be due to the relatively larger access to capital funds to fund research projects, as well as the larger number of employees at their disposal to act as participants. This oversight, however, puts SMEs at a disadvantage, since by virtue of their size and lower funding capacity they are often limited in the options available to them to continuously develop their human asset base (Oriaku, 2012; Fox, 2013).

However, given the aggregate population density of small businesses in existence 
globally, and their significant importance to the sustenance of economies relative to their MNC counterparts (Valadez, 2011), there is need to focus on the development of human capital assets (employees) in such organizations. Also, as SMEs are often considered to be the largest employer of human capital in most economies, using strategic tools like training within such organizations, who tend to have less sophisticated management and HR departmental structures, can often have a positive impact, as SMES can often be potentially better positioned to tailor training activities to their employees (Lee \& McGuiggan, 2008; Yess, 2012).

Evidence from DBEIS (2017) also suggests that currently in the UK, SMEs, who individually could have financial turnover as high as $£ 50$ million, employ an estimated total of 16.1 million people, account for $99.3 \%$ of all private sector businesses, $60 \%$ of private sector employment, with a combined private sector turnover of $£ 1.9$ trillion. Also, in terms of innovation, a significant proportion of small businesses have been said to engage in innovative activities, which are a key driver to organizational growth, as well as healthy competition within the varying industries of an economy (McAllister, 2016). Such innovative activities give rise to the increasing proliferation of start-up businesses, with a fairly significant proportion of these still in existence after the first two (2) years in operation and often growing to become established businesses within the economy.

\subsubsection{Personal Milieu and Reflexivity Practice}

Reflexivity practice for researchers is important in helping to balance objectivity and subjectivity. According to Delbridge and Edwards (2012, p.15), "reflexivity is not just about accumulated experiences. The mode of reflexivity indicates how different actors view their social world in terms of specific personal projects which are, in turn, (re-)formed over time". Thus, we often reflect on ways in which bias may be present in our research practices, and that demonstrates our awareness of this, which helps us rationally seek out precautions we have taken to minimise influence of these biases, in our eventual research outcomes (Jones, Nast \& Roberts, 1997). Therefore, I provide a summarised personal reflective account of my research journey in this sub-section 1.5.1, as well as providing further illumination for some methodological choices made in my study. 
As a qualitative researcher, a key rationale for the use of IPA approach in my research resonates with my decision to make some research methodological choices based on my 'own lived work experiences' as a small business employee spanning a considerable number of years in various SMEs across two continents. I recognised the need for organizations to be able to effectively tailor their training activities, to strategically fit with the objectives of the business (Gratton et al., 1999) and thus produce employees with credible OI status.

My research journey started with an intent to situate my research within the small business research context as well as explore the significance of employee training receptiveness to organizational performance. However, this intent evolved over the course of the earlier two years of my study into the exploration of the Ol literature from where I was able to identify a research gap as to how employee training could help influence this OB construct.

The two elements of SMEs as a site of research and the use of training as a probable organizational interventional or developmental tool for employees are arguably the only elements that remained significantly constant throughout the evolution of my research to completion of my thesis. I also chose to focus on small businesses as the site of my primary research based on my own lived work experiences in similar businesses across different continents, which highlighted the role misalignment of organizational objectives and individual employees' career expectations plays in encouraging the bleed-out of highly competent staff members. This is often apparent in the organizational activities or in-activity the organizations engage in or avoid respectively.

Interpretation of my 'own lived work experiences' in small businesses also suggested that employees in these businesses often tend to be most committed to their companies, with statistical performance optimal irrespective of the remuneration, when they derive satisfaction from their work environment consisting of welcoming colleagues and motivating company leadership. This satisfaction, when it persists, begins to shape engaged employees with a sense of belongingness to the organizations, who feel they essentially belong with the organization rather than just 
being nominal workers in the organizations, hence substantiating the need for developing the underlying notion of organizational identification or, if the reverse is the case, dis-identification.

Fast forward to about midpoint in my research, I happened upon organizational identification, which is the key organizational behavioural construct I have explored in my thesis, while reviewing various $\mathrm{OB}$ literatures to understand how to ensure originality in my doctoral thesis.

Since then I have lived and breathed everything OI using primarily the theoretical understanding of sense-making and interpretative phenomenological analysis as guides in the generation and analysis of my data set via interviews sessions. Thus, the development of my thesis title: "Organizational Identification: Exploring the use of Training as an Employee Identification Marker in SMEs ". With the thesis title now developed, my focused intent was on exploring the role training as an HRM tool could play in determining the Ol status of employees in SMEs.

However, while considering possible eventualities of training for employees' OI process I was well aware that there may be other factors evidently present that could be responsible for narrated employee Ol statuses. Thus, I incorporated a number of other contributory factors in my initial conceptual framework based on evidence from previous literature studies, such as horizontal and vertical communication, organizational culture, motivation and employee unlearning.

Due to the nature of the OI concept as a psychological construct, the interviews were fairly lengthy and these I manually transcribed before analysing the data set from which the conclusions of the research emanated.

\subsection{Significance and Contribution of the Study}

In line with the Interpretative Phenomenological Approach(IPA) methodological stance this research employed, which is fundamentally interpretative in nature, the theoretical background for the study hinged on the application of a hybrid framework based on the sense-making theories from both Karl Weick's and Brenda Dervin's sense-making models to primarily shape employees' OI process within organizations. 
The rationale for adopting both Weick(1993) and Dervin's 1999 sense-making models is that each helped in capturing differing sense-making perspectives, which when combined gave a holistic view of the process of sense-making and its application within an organization. Thus, the derived framework helped facilitate understanding of the participants' experiences and actions, within the defined organizational environment. From this understanding, meaningful assumptions were then interpreted, relative to the research participants' perceptions of their respective Ol processes, alongside training interventions deployed in the workplace.

The final revised conceptual framework proposed in the thesis showcased the possible relationship that exists between various parameters active within a typical employee organizational identification process within their organization. While the initial framework is further explained in the literature review chapter, the framework indicates that 'employer situational-context markers' and 'employee situational-gap markers' [Emphasis Laid] are the two main categories that shape the employee OI process within small business organizations.

Furthermore, the framework suggests that the efficacy of training interventions as a strategic HRD tool can significantly influence the on-going interactions between these factors within the employee OI process. The framework also assumes that the result of any typical employee OI process within small businesses can logically produce only two possible outcomes: 'employee identification' or employee disidentification' (EOI or EOD respectively) relative to the purpose and business goals of the organization, which essentially aligns with Gratton et al.'s (1999) opinion on the strategic fit of human resource management and developmental practices such as training.

A final review of the conceptual framework, detailed in the concluding chapter, shows some convergence between factors identified from Ol extant literature and study participants' experiences, as well as the inclusion of some emerging themes, evident within the context of the study sample employed during the process of this research.

\subsection{Research Sampling}

Within the context of UK SMEs, fifteen participants across a diverse range of economic sectors are interviewed, using a semi-structured format to capture interviewees' perceptions of their organizational identification from their experiential 
stories or narratives.

The approach underpinning the participant selection is purposive and data analysis originates from the Interpretative Phenomenological Analysis developed by Adam Smith in 1998. Using this idiographic methodological perspective and procedural steps, my interpretative analysis systematically construes the interviewees' stories and narratives to gain an in-depth understanding to the OI construct under study and make meaningful assumptions.

\subsection{Thesis Structure}

The structural flow of the thesis is briefly described within this section.

Chapter one defines the introductory scope, rationale and background settings from which the ideas for the research originated. It also highlights the primary aim, objectives and questions the research attempted to address.

Chapter two explores related literatures around organizational concepts such as organizational identification and training. The chapter also explains the theoretical background on which the research is built, as well as elaborating the initial conceptual framework.

Chapter three explores the methodological perspective adopted in the research. Sections covered include the epistemological and ontological background of the research; the phenomenological perspective employed; the research design; as well as limitations and ethical considerations employed.

Chapter four focuses on presenting the research findings, as found from the collated primary textual data, using the thematic techniques prescribed by the principles of the IPA method employed. The material is structured around the research aim, objectives and questions, expressed as sections of the conceptual research framework. The chapter progresses to provide more analysis and discussion of findings adding an additional layer of analysis, by rationally interpreting the underlying meanings for the two Ol markers categories, as evident within the conceptual research framework. 
The research concludes in chapter five with a summary of the findings and how they address the research aim and objectives. This chapter highlights the thesis' contribution to knowledge in terms of the two Ol categories of employer situationalcontext and employee situational-gap markers within SMEs, within the limits of the research participants' stories. Chapter five concludes with a revised construction of the initial research conceptual framework; it also highlights areas for future OI research and draws policy implications from the research findings.

\subsection{Definition of Introduced Terminologies}

Some terms have been used in this thesis to delineate certain conceptual meanings within the confines of this research. Thus, to provide the necessary clarity relative to the research context and OI subject understanding, each of these terms is explained below.

\subsubsection{Organizational Identification Markers}

This term, as used within this research, relates to the factors highlighted by employees as influencing or determining their $\mathrm{Ol}$ statuses within their organizations. That is, they are the factors categorised as either employer situational-context or employee situational -gap OI factors as indicated within the conceptual framework and have been used contextually in various sections of the thesis narrative to fit standard grammatical structures.

\subsubsection{Employer Situational-Context OI Markers}

As used within this research, such markers refer to organizational factors that influence an employee's Ol process within a defined organization. They relate to factors such as vertical and horizontal communication, as well as leadership and organizational culture that employees experience and react to. It is assumed that they are external factors to the individual employee and that the employee is unable to exert any control over these factors. That is, employees are only participating actors relevant to the implementation of the concepts within their small businesses.

\subsubsection{Employee Situational-Gap OI Markers}

These factors refer to organizational identification determinants that are factors the employee within small businesses can regulate or control, as they are often cognitive 
elements situated within the mental framework of the individuals or concrete activities they are able to make a participatory choice on. That is, they are able to choose whether or not to participate in these physical activities. For example, an employee's career aspiration is dependent on the individual and each employee has their own uniqueness relative to this aspiration. Another example could be training activities facilitated by small businesses.

\subsubsection{Stories or Narratives}

Within the context of this research, the terms stories and narratives, as used in relation to employees, connote the recounted and lived experiences of the research participants shared with the researcher during the pre-, during and post-interview sessions. They are the main primary data that are analysed to understand and explain the participants' perception of the Ol construct. 


\section{CHAPTER 2}

\section{Literature Review}

".The qualitative researcher's perspective is perhaps a paradoxical one...although a researcher's knowledge is always based on his or her positionality... it is to be acutely tuned-in to the experiences and meaning systems of others to in dwell and... we have an appreciation for the fluidity and multilayered complexity of human experience....at the same time aware of how one's own biases and preconceptions may be influencing what one is trying to understand...".

(Mullings, 1999 and Maykut \& Morehouse, 1994 both cited in Dwyer \& Buckle, 2009, pp. 55-60)

\subsection{Organizational Identification Construct}

Over the past couple of decades, a considerable amount of research has been dedicated to exploring the often-complex relationship between employees and their employing organizations. Stinglhamber et al. (2015) noted varying academic viewpoints of this complex relationship across various sectoral disciplines including business, Information and Communication Technology sciences as well as the health sciences.

One such viewpoint is from an organizational behaviour (OB) perspective and it relates to the psychological relationship between the employee and the employing organization and has thus been conceptualised in terms of organizational identification (OI) (Bacaksiz, Tuna \& Seren, 2017). Such Ol studies' outcomes indicate that OI does have a considerable effect on aspects of employees' behaviour such as their performance or output (Frone \& Finn, 2004; Riketta, 2005; Carmeli et al., 2007; Walumbwa et al., 2008).

This chapter focuses on reviewing and analysing the various research in the past four decades that has been instrumental in the evolution of what the understanding of OI process is as we know it today within organizational behaviour literatures. The chapter also explores relevant extant literature that tries to substantiate possible 
evidence indicating the plausibility of using training as a human resource management and developmental tool to influence employees' Ol behaviours.

\subsection{Relationship between Identity and Identification}

The main thrust in this study is to understand the meaning of OI from an SME perspective. However, the similarity and interconnectedness between the two constructs of Identity and Identification necessitates the need to understand what both terms mean individually (Ashforth, Harrison \& Corley, 2008).

Defining identification in 1987, Cheney and Tompkins stated it is the appropriation of identity, either by the individual or collective in question or by others. It is essentially the development and maintenance of sameness against outside elements within a specified individual or a group; that is, possession or imbibing of certain attributes and characteristics that distinguish you as a unique individual or association with a group. Such uniqueness or differences displayed represent the individual's or group's identity, while identification relates to the process of developing the identity. Identification is a process (often gradual) and its root or nature is continually evolving to mirror the current psychological state of its possessor, be it individuals or groups of people (Cheney \& Tompkins,1987; Garrety, 2008)

Identity and identification are central to the questions of 'who am I?' and 'what is my role in this world?' (Albert, Ashforth \& Dutton, 2000). Thus, an understanding of identity (often synonymously referred to as self-identity when related to an individual as opposed to an identity developed as a group) is imperative. This is important, as today's growing body of academic research on practices of HRM and employee identity studies in organizations seem to have grown attentive to employees' activities of defining self-identity as being considerably associated with desirable outcomes such as better work performance, organizational citizenship behaviour and the retention of workforces (Mael \& Ashforth, 1995; Abrams, Ando \& Hinkle, 1998; Dukerich, Golden \& Shortell, 2002; Alvesson \& Willmott, 2002; Karreman \& Alvesson, 2004). For example, regulating the self-identity of employees can serve as a form of internal and more effective mechanism in comparison to external stimuli which often ineffectively control the internal elements of the individual such as "the hopes, fears, and aspirations" of employees (Alvesson \& Willmott, 2002). 
This aspect of organizational control itself is not however devoid of criticisms by ethical business scholars, with a key argument being that of accusations of cultural engineering whereby employees' emotions, beliefs and values are altered (Willmott, 2003) and they can become increasingly anxious, burnt-out and exhibit falsified behaviours or inauthentic play-acting (Casey, 1995; Collinson, 2003; Hochschild, 2003; Kunda, 1992; Sennett, 1998). Supporting the use of organizational control mechanisms, however, it has been argued that employees cannot be easily influenced, they can and do resist attempts at manipulation by constructing and maintenance of robust opposing identities (Ackroyd \& Thompson, 1999; Collinson, 1994; Ezzamel et al., 2001; Thomas \& Davies, 2005), and that employees can sometimes even use these attempts at manipulating them to their own benefit. (Robertson \& Swan, 2003; Rosenthal, 2004).

This research study, however, explores the viewpoint of Garrety (2008), on the control-self dynamic within organizations, which rather than pursuing the scholarly argument further, considers a more beneficial endeavour would be to explore the usage or non-usage, as the case may be, of these organizational control mechanisms, specifically "What values and models of the self are implicit or explicit in ... control, and how can these be mobilised to sharpen our analyses?" (Garrety, 2008, p. 93).

Identity has over time manifested in three different contexts within scholarly literature: socially as social identity theory, self-categorisation theory; structurally as structural identity or identity control theory; and from an organizational perspective as organizational identity and corporate identity (Hatch \& Schultz, 1997; Podnar, Golob \& Jancic, 2011).

Social identity is "the part of the individual's self-concept which derives from his knowledge of his membership of a social group (or groups) together with the value and emotional significance attached to that membership" (Tajfel, 1981, quoted in Ashforth, Harrison \& Corley, 2008).

Identity theory refers to the idea that people attach different meanings and significance to the various roles that they play in "highly differentiated societies" (Ashforth et al., 2008). Thus, asserting that identity can become fractured or 
fragmented, making individuals exhibit different identities, given different contexts; i.e. the theory explores roles, such as one's occupation and group association.

Corporate identity is distinct from organizational identity in that it is presumably considered to be more concerned with the visual superficial and graphic identity an organization tries to create and hinges mainly on the role and function of leadership within the organization. This visual representation of the company is often from external parties such as clients and customers (Hatch \& Schultz, 1997). Organizational identity is however construed to be more concerned with employees' intra-organizational relationships within their respective organizations (Hatch \& Schultz, 1997), that is, how employees perceive their organizations. Famously defined in 1985 by Albert and Whetten, as the "enduring characteristics of an organization that contribute to the distinctiveness and uniqueness of an organization", organizational identity is widely referred to within organizational psychology and management literatures.

\subsection{Organizational Identity}

According to Aust (2004), organizational identity is established through communicated values to internal and external stakeholders (Aust, 2004). Organizations establish and communicate an identity in order to "control...how the organization is commonly represented" (Cheney \& Christensen, 2001). Also, organizational identity is about 'self-referential meaning', that is, 'an entity's attempts to define itself' (Corley, et al., 2006, p. 87), thus implicating questions such as 'who are we?' and 'who do we want to become?'.

Other organizational studies scholars also argued that organizational identity has become an increasingly important area of inquiry (Brown, 2006; Corley et al., 2006), especially in informing management relationships with employees in organizations (Cheney, 1991). Also, Park (2014) explores the ideology of identity gap in the context of workplaces which is often complicated with the continuous dynamism needed in an attempt to distinguish between individualised identities and an individual's identity within an organization. That is, one's individual identity without and within an organizational context. 
In comparison, this resonates fairly with Albert, Ashforth and Dutton's (2000) belief that organizations should know who or what they are, what they are or are not in relation to other entities and what the relationship is between themselves and others in order for one organization to interact effectively with other organizations in the long run: "identities situate the organization, group and person". That is, who or what organizations see themselves as, which is what often drives their activities as a business, is what their identity is. Thus, for employees to form an identification with their companies, these companies need to have a known or apparent and substantive or worthy identities with which their employees can identify. The act of this identification with their respective companies is what the term employee identification refers to, also known as belongingness with the business.

The organizational activities mentioned above are often expressed in terms of set company values, goals, mission statements, both the visible and buried elements of their company culture and visions, thus providing a themed platform for structured communication with employees in fulfilling these set targets. Hence, the more an employee can identify with those communicated values, goals and objectives, the more organizational identification there is. Organizations may therefore be able to increase the chances of organizational identification by conveying and repeating set goals and values that employees not only identify with, but are constrained by, when they make decisions.

Some authors have however disagreed that an identity is enduring but instead concluded it is ever-changing and responsive to its environment in modern organizations (Whetten, 2006). Despite differing understanding around the term (Whetten, 2006), most scholars still agree it is a concept worth talking about. Ultimately, there is an emergent consensus that it is 'because identity is problematic and yet so critical to defining organizational behaviour...that the dynamics of identity need to be better understood' (Albert, Ashforth, \& Dutton, 2000).

In their original formulation of the concept, Albert and Whetten (1985) argued that an organization's identity was constituted by a set of claims regarding what was central, distinctive and enduring about it. However, they did not indicate the criteria for specifying these claims (for example, what constitutes 'centrality'), but did recognise explicitly a number of complicating factors which mean that organizations may be 
characterised by multiple identities and that identities claims are often political acts, can be ambiguous, complementary, unrelated and contradictory.

Unsurprisingly, therefore, the use of the concept over subsequent decades has not been consistent, leading Whetten (2006, p. 220) to complain that 'the concept of organizational identity is suffering an identity crisis' and Pratt (2003, p. 162) to assert that as a concept identity 'is often overused and under specified'. Recognition of these issues has led to multiple attempts to re-define the concept, for example, as 'the theory that members of an organization have about who they are' (Stimpert, Gustafson, \& Sarason, 1998, p. 87) and more recently as 'the combinative construal of firm culture, history, structure, characteristics, status and reputation' (Martin, Johnson, \& French, 2011, p. 576).

Another perspective holds that organizational identity is a figurative notion that suggests similarities between individual and collective identities (Cornelissen 2002), with most scholars preferring to regard it as a phenomenon referring to psychological and social realities with antecedents and consequences for other social processes and organizational outcomes (Haslam, Postmes, \& Ellemers, 2003, p. 359). Also, as stated earlier in this section 2.2, another considerable debate centres on how to differentiate organization identity from cognate terms such as corporate image and reputation (images projected to external audiences), construed external image or perceived external prestige (how insiders believe outsiders view the organization), and particularly organizational culture (Fombrun, 1996; Fombrun \& Shanley, 1990; Gioia, Schultz, \& Corley, 2000; Hatch \& Schultz, 1997). Attempts to address these and other related issues have resulted in a multiplicity of perspectives, with varying ontological and epistemological assumptions and methodological preferences.

Despite these definitional variations, the identity field can still be said to be "riven with uncertainties and often fractious disagreements" (Podnar, Golob \& Jancic, 2011). Thus, this research hopes, by exploring the factors that determine the employees' identification status, to contribute to academic knowledge as to what employees consider to be characteristics of organizational identities they may like to identify with. 


\subsection{Organizational Identification}

Organizational identification is an offshoot of the social identity perspective, which holds the view that individual persons classify themselves and others into social categories, in order to form an identity or exhibit a personality within their immediate social environment (Stinglhamber et al., 2015; Ashforth \& Mael, 1989).

Therein, the individual defines him or herself in terms of the social environment in which they are a member, that is the organization in a business context. This is fairly in line with assertions by various scholars that the organization is one of the most fundamental foci of identification for an individual (Aronson, Blanton \& Cooper 1995; Ashforth \& Mael, 1989). Essentially this suggests that individual employees would most likely self-associate themselves or define themselves and who they are in reference to their respective business organizations.

Defined by Reade (2001) in Podnar, Golob \& Jancic (2011), organizational identification is also said to be the process of psychological bonding between an individual and his or her work organization, which is an important goal in corporate marketing (Balmer, 2009). According to Pratt (1998), OI is an alignment of individual and organizational values. It refers to the perception of oneness with and belongingness to the organization (Ashforth \& Mael, 1989). It has also been defined as the propensity of a member of an organization to identify with that organization and largely depends on how the individual perceives the organization, including its prestige, the degree of communication and support it offers them, and how it presents its corporate brand identity, as well as on the individual's self-perception and his/her own goals and aspirations (Aronson, Blanton \& Cooper, 1995; Ashforth \& Mael, 1989).

Organizational identification is an important field of research with several studies showing that there exists a strong correlational relationship between identification and commitment to the organization itself (Tompkins, 2005; Boros, 2008). These include commitment being the main component that could potentially ensure employees unapologetically stay with their organizations (Tompkins, 2005). These statuses, if exhibited, often produce more engaged and productive employees. 
Furthermore, $\mathrm{OI}$ is known to be synonymous to an individual's view and classification of self in terms of organizational membership (Rousseau, 1998). Social identity theory has also combined the cognitive elements of Ol described above with its affective and evaluative components. For example, emotional attachment, feelings of pride, and other positive emotions that are derived from organizational membership have been incorporated in the operationalisation of OI (Bergami \& Bagozzi, 2000).

\subsection{Key Organizational Identification Schools of Thought}

Though the introduction of the organizational identification notion dates back about three decades, the review of literature dating back about half a century highlights two major Ol schools of thought by Ashforth and Mael (1989) and Dutton, Dukerich and Harquail (1994).

Ashforth and Mael's school of thought exploring the concept of OI in 1989, discusses the interrelatedness and significance of the social identity theory in understanding the OI process. In their OI literature, they refined the concept of identification, differentiating among its cognitive, behavioural and emotional aspects, as well as distinguishing between identification itself and its antecedents or consequences. That is, what causes the occurrence of $\mathrm{Ol}$ and what are the resulting outcomes that may or may not follow once the concept takes root within the individual? Thus, they defined $\mathrm{Ol}$ as the perception of unity with or belonging to a social aggregate. Meaning $\mathrm{Ol}$ is a form or means of self-categorisation within an organization in the context of this research. Explaining this further, as an organizational psychological construct, organizational identification is sometimes not viewed in absolute terms but more as a progressional attribute and an outcome of a cognitive self-categorisation process (Ashforth \& Mael, 1989; Boros, 2008).

Thus, Ashforth and Mael(1989) proposed four conceptual assumptions in an attempt to define and understand the OI process as stated below:

- "Identification is a perceptual-cognitive concept, not necessarily associated with specific behaviours or emotional states...

- Group identification means experiencing at a personal level the group's successes or failures... 
- Identification is different from internalisation. While internalization means incorporating the group's attitudes or values as guiding principles of one's own behaviour, identification means referring to self in terms of a social category. Thus, accepting a social category as a definition of self does not imply also accepting the group's values and attitudes.

Moreover, identification is specific to each organization; internalization and commitment might not be, because several organizations may share common goals and values. Commitment might arise because that particular organization is a vehicle for one's own career goals. This leads to the fact that leaving that organization for another one, where these goals can better be fulfilled, is a possibility at all times. Identification with an organization, however, means one cannot leave it without some kind of 'psychic loss'...

- Group identification is similar to identification with an individual, in the sense that one defines oneself in terms of that social referent..."

Summarising these assumptions, Ashforth and Mael(1989) meant that identification is a function of the individual's mental perception and not necessarily contextually dependent; that group identification can also have an individualised experiential component, inherent in the relevant individual and relative to the reference group's actions or inactions; also, that an individual's identity without the reference group does not necessarily have to be the same or conform to the group's unique identity but identification is more about willingly acknowledging association with the reference; and finally, their fourth assumption asserts that identification is about feeling a sense of belonging with an external entity. Such an entity can be anything ranging from other individual(s) to group(s) or organization(s).

Thus, based on the understanding of the itemised assumptions above, the organizational identification scale (OIS), popularly also known as the Mael scale, was proposed in 1992. This scale was employed for the widely known meta-analysis work of Riketta on OI in 2005. Riketta used the OIS questionnaire in comparison to other OI measurement tools, such as the OI questionnaire and attitudinal organizational 
commitment scales, to explore the correlational relationship that exists between $\mathrm{OI}$ figures and a wide range of work-related attitudes and behaviours such as performance.

One important outcome of the Riketta 2005 study was the result suggesting that individuals with more organizational identification are less likely to leave the organization in which they currently work, i.e. "less intent to leave". Thus, OI was proving to be a good predictor for employee turnover rates within the organization. That is, employees with less organizational identification are likely to be more willing to leave the organization and vice-versa. Riketta's study also establishes the significant differences between $\mathrm{OI}$ and the organizational commitment construct.

Nevertheless, Bergami and Bagozzi (2000) argued that despite Mael and Ashforth's (1992) scale measuring overall OI, it could inadvertently target more than an individual's awareness of their own membership in the organization to also include the potential causes, effects and correlates of the Ol process. That is, the Mael scale has the inherent flaw that it could also be measuring the impacts of other related organizational psychological constructs other than $\mathrm{OI}$ and unknowingly attribute the results as being a causative effect of $\mathrm{OI}$.

A second school of thought deriving from the Dutton et. al. (1994) Ol studies, however, suggested that OI occurs when the following two assumptions hold:

- An individual's organizational identification is more important than alternative identities, and

- The individual's self-concept has many of the same attributes he or she believes characterise the organization as a social group.

That is, they believed that an individual would often have a stable identity once they acquire an identification status. Secondly, rather than Mael and Ashforth's assertion on the possibility of differing identities with or without a reference entity, Dutton and Dukerich (1991). believed that an individual's identity with or without reference to an external entity is more or less likely to be one and the same (Dutton, Dukerich \& Harquail, 1994; Mael \& Ashforth, 1992). 
Thus, defining organizational identification as "the degree to which a member defines himself or herself by the same attributes that he or she believes define the organization". Dutton et. al. (1994) asserted that strong Ol only occurs when members of an organization consciously mimic the characteristics of the organization in their self-concept; the self-concept being "the totality of self-descriptions and selfevaluations subjectively available to an individual" (Hogg \& Abrams, 1988).

Furthermore, Dutton et al. (1994) proposed three means of acting on the idea of identification. Firstly, by directly assessing it, through scale-based measures. Secondly, by tasking organizational members to evaluate a given set of pre-set identities and indicate the relative degree to which these identities accurately describe them as individuals, either by ranking each identity or ranking them in hierarchy. Or thirdly, by directly assessing the level of overlap between the characteristics by which an individual describes him or herself and the characteristics that best describe the organization.

\subsection{The Then and Now Evolution of Organizational Identification}

Research from various OI literature has suggested that $\mathrm{Ol}$ is the bedrock for many observable organizational outcomes (Haslam, 2001; van Dick, 2001). However, the persistent question arising in the Ol field of study is often centred around how exactly organizational identification can be increased in organizations to deliver these associated positive organizational results (Peters et al., 2012).

To date, several alternatives have been offered to address this issue, as will be discussed in further sections of this chapter 2. For example, one solution recommended is that organizational leaders should encourage common in-group identities (Gaertner et al.,1993), or that organizational activities should be structured in ways that make shared identities important and contingent to successful business outcomes (van Dick et al., 2005) or that communiques from these organizational leaders should define their organizations in a positive, unique and stable manner (Albert \& Whetten, 1985).

Building on the OI work of both 20th century authors, with Ashforth and Mael's work focusing mostly on the act of idea of self-categorisation and that of Dutton et. al. (1994) on social categorisation, both works echo the social grouping categorisation 
perspective on $\mathrm{Ol}$ and this will be the perspective adopted by this research in undertaking the study analysis.

In terms of knowledge development in the field in the last decade, however, the conceptualisation and operationalisation of $\mathrm{OI}$ has undergone significant changes leading to the development of three further $\mathrm{OI}$ schools of thought. The first challenges the initial definition of $\mathrm{Ol}$ in positive terms, as an affirmative relation between the individual and the organization, introducing concepts such as disidentification (Dukerich et al., 1998; Elsbach \& Bhattacharya, 2001; Kreiner \& Ashforth, 2004).

Developed from Dutton, Dukerich, and Harquail's (1994) idea about OI, it is defined as the degree to which a person defines him or herself as not having the same attributes that define the organization. The dimensions of $\mathrm{Ol}$ were obtained and with the assumption of $\mathrm{Ol}$ through the high-low superposition of attributes between the self and the organization (Dukerich et al., 1998; Ellemers et al., 2002; Kreiner and Ashforth, 2004). The three concepts introduced were: disidentification, ambivalent identification, and neutral identification.

Disidentification occurs when individuals do not identify with the organization, ambivalent identification when the employee has both identification and disidentification simultaneously within the organization, and neutral identification when there is explicit absence of both identification and disidentification with the organization (Kreiner \& Ashforth, 2004).

\subsubsection{Organizational Disidentification, Ambivalent Identification and Neutral} Identification

Organizational disidentification is defined as "a self-perception based on a cognitive separation between one's identity and one's perception of the identity of an organization, and a negative relational categorization of oneself and the organization" (Elsbach \& Bhattacharya, 2001). A disidentified member maintains "a sense of selfdistinctiveness through perceptions and feelings of disconnection" (Elsbach \& Bhattacharya, 2001). In other words, disidentification is a self-categorisation in the form of cognitive dissociation from the group or organization. Regarding the dynamic triggers of social categorisation, disidentification is related to the predominance of 
one's needs for distinctiveness over one's need for inclusiveness within the group or organization.

According to Elsbach and Bhattacharya (2001), the antecedents of disidentification include: the perception that one's personal values conflict with the values of the organization; perception that an organization's reputation might affect one's social identity; perception that the members of the organization are "all the same"; and perceptions of the organization that are based on a lack of personal experience with the organization or its members.

Consequences of organizational disidentification in general include: counterorganizational action, organizational criticism, and disidentified members tending to rebel and resist organizational initiatives and goals just because they have been proposed by the organization. These members may also generate a presumptive distrust among other members of the organization and over time valid criticisms may be raised that would likely be given little or no attention (Dukerich, Kramer \& McLean-Parks, 1998).

Neutral identification, on the other hand, is when a person neither connects nor separates his or her identity from the organization, or, at the extreme, does not even have an opinion or knowledge about the organization (Dukerich, Kramer \& Parks 1998; Kreiner \& Ashforth, 2004;). It is also a state of "non-existent or broken identifications" (Pratt, 1998). While an individual with an ambivalent identification combines attributes of both disidentification and identification harmonisingly.

Ambivalent identification is a dual state of both identification and disidentification with an organization. It can take the form of identifying with certain dimensions or traits of the organization's identity, or of simultaneous identification and disidentification with the same traits (Kreiner \& Ashforth, 2004). Extant literatures identified certain triggers of ambivalent identification such as incompatibility of the organization's identity and the negative image of the organization (Kreiner \& Ashforth, 2004; Elsbach \& Bhattacharya, 2001).

Also, based on previous studies such as Ellemers, Spears, \& Doosje (1997) that suggested the superiority of subjective conditions, that is, high initial levels of 
identification over lower levels, it was proposed that the emergence of ambivalent identification in situations of affected organizational image or prestige occurs mainly in those who have an increased need for identification. In the case of low markers, the same objective situation will induce a subjective reaction of breaking up with the organization, either in the form of disidentification or neutral identification.

Ambivalent identification, as argued by Festinger (1957) and Heider (1958) (both cited in Boros, 2008), is however said to be an 'unsafe state of mind', as it is a state of mind that cannot be endured for a long time, which means that eventually one will try to find a strategy or a definite identification type to fit into or come to terms with. Thus, both authors, similarly to cognitive dissonance theories, predict a change of attitude that might solve the cognitive conflict. Self-affirmation theory (Steele, 1988) also argues that if dissonance is aroused by threats to one's general sense of self integrity, then people can effectively respond to these threats in ways not documented by the early dissonance researchers - that is, by affirming some other valued aspect of the self-concept not necessarily related to the threat.

For example, compensating one's behaviour with another expressed behaviour. That is, rather than admitting a change in their own usual behaviour associated to their identification status shift, employees may try to implement other behaviours to seemingly cover up their behavioural change. For instance, an employee justifying that: although I'm often late to work, I complete my scheduled daily duties before leaving the work premises.

More recent studies, however, argue that these compensation and self-affirmation behaviours do not necessarily occur sequentially but could also occur concurrently (Aronson, Blanton, \& Cooper, 1995). As an offshoot of ambivalent identification, individuals can be motivated not only to make excuses for their behaviour, but given certain circumstances, may incorporate these excuses into their self-image. What appears is an interaction between the multiple paths of self-affirmation, in which individuals not only change their attitudes towards the dissonant-arousing object (in this case the organizational objectives), but also change relevant attitudes towards themselves as well within the context of the organization. For example, blaming their poor performance at work on management action they are thus never interested in putting themselves forward for developmental opportunities. 
This interaction can explain why, in time, ambivalent identification can turn into disidentification, and how individuals who initially rejected disidentification get to feel at ease with it by changing their very need for identification with the organization. In this way, changes will occur both in attitude (one decides to change the manner of relating to the organization, by defining oneself through opposite attributes from those used to define the organization), and in the self-concept, through a decrease in one's general need for identification (which would give the final rationalisation as to why they disidentify with the organization). This state is what is inherently meant by employee disidentification, identified within the conceptual framework included later in this chapter.

Thompson and Holmes (1996), however, suggest that the ambivalent state of identification can still be sustained for a limited period, worthy of mentioning, but simultaneously recognise that it is a transient state of identification.

\subsubsection{Organizational Identification as a Social Identity Construct}

Another school of thought on OI explores further the Social Identity theory research and opposes the definition of organizational identification by Ashforth and Mael (1989) as an exclusively mental concept as compared to Tajfel's initial definition of social identity which also comprises an emotional and an evaluative side (Ellemers, Kortekaas \& Ouwerkerk, 1999; Bergami \& Bagozzi, 2000).

According to the primary definition proposed by Tajfel (1978, p.63), social identity is "that part of an individual's self-concept which derives from his knowledge of his membership of a social group(s) together with the value and emotional significance attached to that membership".

Based on Tajfel's definition, Ellemers, Kortekaas, and Ouwerkerk (1999) proposed that three components contribute to one's social identity: "a cognitive component (a mental awareness of one's membership in a social group - self-categorisation), an evaluative component (a positive or negative value connotation attached to this group membership - group self-esteem), and an emotional component (a sense of emotional involvement with the group - affective commitment)". 
The aforementioned authors first distinguished cognitive awareness of one's group membership per se (self-categorisation) from the extent to which one feels emotionally involved with the group in question (affective commitment). This distinction is based on empirical evidence proving that people who belong to the same social group may show differential responses, depending on the extent to which they feel affectively committed to that group ( Branscombe \& Wann,1994; Doosje, Ellemers, \& Spears, 1995; Ellemers \& Van Rijswijk, 1997). While Ellemers et al. (1999) maintained the term 'group commitment' to refer to the emotional attachment towards a social group and used their own measure of this construct, other researchers (Bergami \& Bagozzi, 2000) preferred the term 'affective commitment' to describe emotional attachment to the organization. These authors used affective commitment in Meyer and Allen's (1996) terms (i.e. as identification with, involvement in, or emotional attachment to the organization) and used the affective commitment scale to assess the emotional side of organizational identification (Bergami \& Bagozzi, 2000).

Ellemers, Kortekaas, and Ouwerkerk (1999) differentiated the extent to which people feel emotionally involved with their group (affective commitment) from the value connotation of that particular group membership (group self-esteem). In summary, the Ellemers, Kortekaas and Ouwerkerk (1999) conceptual analysis implies that selfcategorisation (the cognitive component) as well as affective commitment to a specific group (the emotional component) can be distinguished from group selfesteem derived from the value connotation of that particular group membership (the evaluative component).

In addition to arguing for conceptual distinctions among the components of social identification, the authors suggested that these identifiable components are empirically distinct and differentially affected by relative status and size of the group and the basis of group formation: relative group status affects mainly the evaluative component of social identity (group self-esteem), while relative ingroup size affects mainly the cognitive component or self-categorisation aspect of ingroup identification. A consequence of these differential effects is that if it is assumed that low group status negatively affects only the evaluative component of identification, while the level of affective commitment (the emotional component) can remain unchanged, it 
becomes clear that it is the combination of a threat to group self-esteem and strong affective commitment which should elicit attempts to depict the ingroup in a positive way.

Furthermore, the authors suggested that cognitive organizational identification is expected to indirectly affect citizenship behaviours within the organization through affective commitment and group self-esteem. In an attempt to shed more light on the concept of organizational identity, Ellemers, Spears and Doosje (2000) stressed the importance of keeping separate the notion of identification as a cognitive state of self-categorisation from the process of comparing personal attributes with organizational attributes. This will help differentiate the process of identification from its originating organizational identity.

A more recent theoretical review from Ellemers, De Gilder and Haslam (2004) advances the three psychological processes that ultimately lead to the three dimensions of organizational identity, as defined by Ellemers, Kortekaas and Ouwerkerk (1999). They build on the work of Tajfel (1978) and Tajfel and Turner (1979) (both cited in Humphrey 2012, pp. 251-255), who specified three psychological processes that underlie these group-based interactions: social categorisation which is cognitive in nature and social comparison and social identification which are affective and evaluative components respectively.

Social categorisation refers to the fact that people organise social information by categorising individuals into groups, which enables them to focus on the collective properties that are relevant for that particular situation or context. Meanwhile, social comparison is the process by which a social categorisation is invested with meaning. By this process, people determine which features or behavioural norms help to define the group in a particular situation, so as to distinguish it as much as possible from other relevant comparison groups. Thus, what defines members of the group may vary from one situation to another, depending on the situational context and the ways in which group members are distinct from others in that context.

Social identification, on the other hand, is the process by which information about groups is related to the self. It refers to the inclination of a particular individual to 
perceive themselves as an ambassador of a particular group that is the organization to which they belong, which makes the individual perceive characteristic group features as self-descriptive and leads him or her to adopt distinctive group norms as guidelines for his or her own behaviour that is identifying with the organization. Social identification is often used to refer to a feeling of affective commitment to the group which is the emotional component rather than the possibility to distinguish between members of different social categories (the cognitive component) (Ellemers, Kortekaas \& Ouwerkerk, 1999).

Furthering on this, Ellemers et al. (2004) emphasise that the cognitive tool of social categorisation and the evaluative feature of social comparison processes can influence a person's involvement with a particular group and their sense of identification with that group.

Thus, while Mael and Ashforth's (1989) definition of OI stops at the primary process of self-categorisation (and portrays the evaluative and emotional dimensions as consequences), Ellemers et al. (2004) encompass in the same construct all three aspects of identification regardless of their chronological occurrence. In other words, while Mael and Ashforth (1989) were more concerned with the sequence in which the identification process occurs, Ellemers et al. (2004) focused on the conceptual integration of the varying elements of the process.

\subsubsection{Organizational Identification as a Multi-Factorial Construct}

Although the Ol approaches discussed above appear logical, Peters et al. (2012) highlighted some significant inadequacies in them. Firstly, they seemingly paid minimal attention to the important fact that organizations as entities are not the sole sources of social identities available to organizational employees. Meaning that employees in organizations may sometimes define themselves in terms of less abstract, lower-level identities with intra-organizational groups such as a work unit or department, rather than with the organization holistically, with whom their existing relationship may be unstable, problematic or uncertain (Ashforth, Harrison, \& Corley, 2008; van Knippenberg \& van Schie, 2000). An attempt was made by Gaertner, Dovidio \& Bachman (1996) to address this limitation by introducing the notion of dual identity. However, Jetten, O'Brien and Trindall (2002) contest this by highlighting that 
alternative identities could actually create significant obstacles to organizational identification.

A second limitation identified is that more often than not solutions provided for improving or increasing organizational identification are often politically skewed or challenging. This is because they are often expressed from the perspective of organizational leaders, which often pays minimal or no attention to the differing viewpoints and aspirations of the employees, who are themselves the actors of organizational identification (Haslam, 2001; Tyler \& Blader, 2000). Thus, these perspectives are seen to be flawed, since leader-centric strategies likely to be proposed to build organizational identification in such companies are more likely to be met by employees with resistance or cynicism. This is often because such strategies may be seen to be contra-indicative to employees' alternative valued identities (Brehm, 1966; Kelly \& Kelly, 1991 both cited in Peters et. al. 2012).

This thus raises another question about the efficacy of proposed strategies for organizational identification strengthening in companies, and their potential outcomes. Hence, a third more recent viewpoint on organizational identification emphasises the significance of predicting subsequent behaviour of individuals, which changes the focus of $\mathrm{OI}$ to a multi-level analysis, starting from the work-group, moving up to the organization and finally the occupation of the individuals (van Knippenberg \& van Schie, 2000; Riketta \& van Dick, 2005; van Knippenberg \& Sleebos, 2006).

This research thesis has placed much emphasis on this third school of thought, viewing $\mathrm{OI}$ as a multi-level psychological construct which is expressed by the individuals across different hierarchy levels in an organization and bounded by the organizational boundary itself. This is in alignment with the primary aim of the research, which is to explore the influence organizational interventions (with emphasis on training activities) have on employee OI.

Borrowing ideas from other organizational identification schools of thought, however, this research also explores ideas from such as social identity theory research (that suggests identification and disidentification are negatively correlated, but not exact 
opposite concepts). Explaining this further, the major difference between the two is that disidentification involves either extreme and/or simplified perceptions of the relationship between one's identity and the identity of an organization (Ellemers, Kortekaas \& Ouwerkerk, 1999; Abrams \& Hogg, 1999). In agreement with this, Ellemers, Kortekaas \& Ouwerkerk (1999) further opined that disidentification, understood as cognitive distancing of a social identity from a group identity, is due to the perception that one is distant from the group prototype or norm. Thus, it is assumed that disidentification with a group might be a defence mechanism used by low-markers to pre-empt rejection from the group, or by those who want to integrate themselves with a more desirable group.

However, Elsbach and Bhattacharya's (2001) study did not investigate the mechanisms that produce disidentification, but considered this as an area for further research. Thus, this research sets out to discover some of the elements that may most closely mean or relate to these idealised mechanisms as factors that influence an employee to exhibit employee organizational disidentification status within their organization.

\subsection{The Strength of Organizational Identification}

As earlier discussed in sections 2.1 and 2.2 (pp. 20 \& 22 respectively), Albert, Ashforth and Dutton (2000) construed identity and identification as "root constructs in organizational phenomena" and as concepts that form the bedrock on which many observable organizational behaviours are built. Also, several other extant literatures emphasise that individuals do not just casually identify with social groups as an instrument for self-enhancement, only to ditch these groups when they no longer derive this from them. Rather, individuals continue to identify with these groups even through the bad times, when the negative image (or the low status) of the group actually affects their need for self-enhancement (Turner et al., 1984; Branscombe \& Wann, 1999; Ellemers, Spears, \& Doosje, 1997).

This does not occur because of objective conditions only (for example, low group permeability represents the impossibility of leaving the group due to such as power distribution in the organization, fear of unemployment, etc.), but also in combination with other subjective factors such as initial levels of identification (Doosje, Ellemers, 
\& Spears, 1995). Different combinations of these two types of factors result in varying levels of organizational identification.

\subsection{Organizational Identification Process: Control or Regulation?}

It is said that the strength of an employee's identification with an organization often guides or constrains that employee's decision making and that Ol could act as a form of organizational control and this happens when "a decision maker identifies with an organization and desires to choose the alternative which best promotes the perceived interests of that organization" (Cheney \& Tompkins, 1987).

For example, Cheney (1983) states that organizational policies actually affect and shape the development of identification "in terms of what is communicated to the employee". As employees they can only identify with those things that they are aware of, and many of the things they are aware of and how they understand those things are communicated to them by the organization in predetermined ways.

It is also right to mention that companies using organizational identification could steer behaviour by influencing which problems and alternatives are seen and by postulating biased choices that appear most crucial to organizational success (Kassing, 1997). Organizations can choose to emphasise particular problems and alternatives through communicated goals and values, causing employees to identify mainly with those communicated goals and values. This then limits their choices and constrains their decision making in a way that is positively aligned with the organization's goals and values. Thus, aware of the benefits to themselves, the management in such organizations intentionally foster OI among their employees by "identity regulation" and as a sort of organizational control.

Issues of control are found in most activities implemented at most levels of organizational life (Larson \& Tompkins, 2005). Organizations can exercise simple control (direct, authoritative), technological control, and bureaucratic control (through rules and rationality). The most powerful forms of control in an organization may be those that are the least obvious or "that are 'fully unobtrusive' that 'control the cognitive premises underlying action"' (Perrow, 1979, quoted in Larson \& Tompkins, 2005). 
Barker (1993) calls the control described above "concertive" control which he argues largely develops from self-managing teams who base decisions on a set of shared values and high-level coordination by the team members themselves. Concertive control, even though employee directed, actually increases the total amount of control in an organizational system because each worker is watching and correcting others (Tompkins, 2005), rather than one manager watching and directing the behaviour of many.

At the extreme of organizations demonstrating control is, however, the attempt to regulate employee identity and identification. Alvesson and Willmott (2001) explored how employee identities are regulated within organizations, so that their self-images, work processes, outcomes and products line up with management goals and objectives.

Identity regulation is the "intentional effects of social practices upon processes of identity construction and reconstruction" (Alvesson \& Willmott, 2001). The authors suggest that when an organization and its rules and procedures, particularly in training and promotion activities, become "a significant source of identification for individuals" the organizational identity is then at the core of that individual's "selfidentity formation process and simultaneously acting as a subtle control mechanism (Alvesson \& Willmott, 2001). The conscious effort, either by the organization or the individual, to align self-image with organizational goals is organizational identification, and OI can bound an employee's decision making in a way that keeps it "compatible with affirming such identification" (Tompkins \& Cheney, 1985).

Organizations can manage OI by managing how individuals form personal values and identities, and how those values cause them to approach relationships inside and outside of work (Pratt, 2000). Organizations can do this by "creating a need for meaning via sensebreaking" (Pratt, 2000) by causing people to question their 'old' values against the new, better values and incentives offered by their organization.

So, influencing employees' Ol process could immensely benefit the company because it makes for more satisfied employees who stay longer and work harder. Identity regulation by organizations can be seen in their efforts to manage organizational culture through communicated values in mission and vision 
statements. Organizations can also create a vacuum and then a perceived need among employees for goals and values provided by the organization through sensebreaking and dream-building (Pratt, 2000).

Finally, organizations can attempt to shape the values and identities of the workforce through self-help programmes selected and instituted by the organization in the workplace, although controlling exactly how these programmes are interpreted and applied can be difficult (Carlone \& Larson, 2006). Examples of such self-help programmes include HRM activities such as trainings organised by the companies and geared towards legal compliance requirements as well as employee professional development when needed.

\subsection{Implications of Organizational Identification.}

OI has been associated with a number of positive organizational outcomes, such as work attitudes and behaviours including motivation, increased job performance and satisfaction, individual decision making, and employee interaction and retention (Cheney, 1983; Scott, Corman \& Cheney, 1998; Ciftcioglu, 2010; Haslam, 2001; van Dick, 2001). Some of these outcomes, such as good employee satisfaction and retention figures, have implications for productivity, efficiency, effectiveness and ultimately profit within the organization. This supports the notion that organizational identification is so significant that employees who possess strong identification may even set aside their own self constructed moral standards for the sake of the organization, which showcases a sacrificial behaviour.

Furthermore, organizational identification is mostly bench-marked as an individual's view and classification of self in terms of organizational membership (Rousseau, 1998). Social identity theory has combined the cognitive elements of organizational identification described above with affective and evaluative components. For example, emotional attachment, feelings of pride, and other positive emotions that are derived from organizational membership have been incorporated in the operationalisation of organizational identification (Ashforth, Harrison \& Corley, 2008).

According to Ashforth, Harrison and Corley (2008), there are four major reasons why organizational identification is important. Firstly, its significance to the concept of self- 
identity, which is one way in which people come to define themselves and make sense of their place in the world as well as appropriately traverse their worlds.

Secondly, the essential need exhibited by humans as a longing to identify or have a sense of belonging with a larger group which OI seems to efficiently satisfy. The third reason being Ol's strong correlation to a number of important positive organizational outcomes, including employee satisfaction, performance and retention. In addition, despite the recent studies that had explored the potentially negative outcomes of $\mathrm{OI}$, including reduced creativity and resistance to change, some new insights into the nature of the relationship between $\mathrm{OI}$ and other organizational behaviours such as leadership, perceptions of justice and the meaning of work are becoming more evident (Ashforth, Harrison \& Corley, 2008).

Also important is the link between company policies and rules, communicated mission, values and strategy, and organization members' attitudes and the strength of an employee's identification with the company (Cheney, 1983). This notion opened the field of organizational identification to studies and questions about organizational control of employees through efforts to increase or improve organizational identification.

\subsection{Organizational Impacts of Organizational Identification}

In organizational identification extant literatures, research narratives have proposed that identification is the bedrock on which employees form attitudes and behaviours towards organizations (Albert, Ashforth, \& Dutton, 2000; van Knippenberg \& van Schie, 2000). This is further supported by a growing number of mostly quantitative studies within the service sector evidencing that organizational identification is positively interrelated with organizational commitment, organizational citizenship behaviour in organizations, job satisfaction, perceived organizational prestige external to the firm, employee performance and laissez-faire leadership and management styles and the employee-employer emotional bond in organizations. That is, the more organizational identification with the organization is portrayed by its employees, the more these associated factors are evident in the company.

However, organizational identification is said to be negatively correlated with employees' turnover intentions (Bomber \& Iyler, 2002; van Dick et al., 2004; Riketta, 
2005; Mignonac, Herrbach \& Guerrero, 2006; Ciftcioglu, 2010; Humphrey, 2012; Xenikou, 2014). That is, the more the organizational identification, the less employees feel the need to leave the business establishments (Santhanam, Kamalanabhan \& Dyaram, 2014). Literature on some aspects of this causal relationship will be explored further within this sub-section 2.9 .

Empirical evidence to date is mostly based on findings supporting the notion that high level of $\mathrm{Ol}$ has a direct positive correlation to increased organizational commitment, job satisfaction and higher employee retention figures. Thus, exploring the dynamics of fluctuating employee Ol levels due to organizational training activities, which is the thrust for this research, will help contribute considerably to address the paucity of literature regarding $\mathrm{OI}$ as an organizational behaviour construct in organizations.

\subsubsection{Organizational Identification and Organizational Commitment}

Organizational commitment and organizational identification have been significantly linked as having a correlated relationship (Ciftcioglu, 2010; Riketta, 2005). However despite the statistically evident similarities between organizational identification and organizational commitment from the above studies, especially in terms of affective commitment, these perspectives have largely developed independently from each other. Scholars who have attempted to compare these two fields of research have also questioned the validity of affective commitment in Ol studies (Becker et al., 1996).

So far, the distinction between these two constructs has been supported at both the theoretical and the empirical level ((Bergami \& Bagozzi, 2000; Boros \& Curseu, 2005; Branscombe \& Wann, 1994). These scholars however agree that despite their correlated relationship, differences exist between these two concepts.

In general terms, organizational commitment is defined as "a stabilizing or obliging force that gives direction to behaviors" (restricts freedom, binds the person to a course of action) within the organization" (Ciftcioglu, 2010). Other meanings of organizational commitment include an "exchange based relationship between individual and working organization" (Hrebiniak \& Alutto, 1972 cited in Ciftcioglu, 2010). Wherein if this exchange process works effectively, a commitment is 
established between the two exchange parties (Blau, 1989; Becker, 1960, cited in Ciftcioglu, 2010).

In psychological researches linking OI and organizational commitment, commitment is said be an "internalization of organizational goals and values, willingness to invest effort in the organization and sense of belongingness manifested as a wish to stay" (Steers, 1977; Porter, et al., 1974; Mowday, et al., 1979, cited in Ciftcioglu, 2010) or "a psychological state that characterizes the employee's relationship with the organization and has implications for the decision to continue membership in the organization" (Meyer \& Allen, 1997).

Convincing arguments have been raised suggesting that commitment binds an individual to an organization and that commitment offers a stable psychological structure that helps maintain employees' interactions and identification with their organizations, especially in deciding whether to remain there (Meyer et al., 1993; Meyer \& Allen, 1997).

In addition, many studies have reported a positive strong relationship between the two constructs (Brett, Cron \& Slocum, 1995). However, despite the attention organizational identification and organizational commitment have received, there is still considerable disagreement regarding how they are related (Becker et al., 1996). First, little attention has been given to assessing the direction of causality between these two constructs and the potential role of one concept in the development of the other. Second, not enough emphasis has been laid on explaining why both organizational identification and organizational commitment have common antecedents and outcomes. Filling these important gaps, Boros (2008) argued that organizational identification which is a psychological cognition-based construct is a determinant of organizational commitment as well.

A summation of other studies also indicates that employee's identification with the organization fosters emotional commitment to the organization (Mael \& Ashforth, 1992; Meyer \& Allen, 1997; Riketta, 2005; van Knippenberg \& Sleebos, 2006; Boros, 2008); that is, individuals who identify with their organization remain in the organization because they "want" to stay. 


\subsubsection{Organizational Identification, Perceived External Prestige, Organizational and}

\section{Corporate Identity; and Organizational Culture}

Developing literature, mostly within the marketing service literature, has increasingly been examining the causative relationship between organizational identification and perceived external prestige. Drawing from the organizational commitment and perceived external prestige literature suggesting that employee's positive prestige perception influences employee organizational commitment to initiate organizational effectiveness (Carmeli \& Freund, 2002; Freund, 2006), March and Simon (1958 cited in Ciftcioglu, 2010) iterate that prestige perception develops an emotional bond with organization and individuals.

Ellemers et al. (1999) and Bergami and Bagozzi (2000) also examined organizational commitment and organizational identification in a perceived external prestige relation model using Tajfel's (1982) three-dimensional identification construct. The empirical findings of Bergami and Bagozzi (2000) showed that "perceived organizational prestige has positive effects on commitment by organizational identification mediating effect". Similarly, to the above, factors such as attractiveness, distinctiveness, prestige, and construed external image have been traditionally construed to be major antecedents of employee Ol (Dukerich, et al. 2002; Dutton, et al., 1994). The general rationale supporting these relationships is that employees are more likely to identify with an 'attractive organization' which has good perceived external prestige. This is because it helps to enhance employees' own self-image (Podnar, Golob \& Jancic, 2011; Farrell \& Oczkowski, 2012).

This perceived external prestige could also be argued to be a component of the organizational and corporate identity employees feel their organizations possess and this identity is often a reflection of the organizational culture of these companies (Podnar, Golob \& Jancic, 2011). Thus, organizational identity is another key element highlighted in extant OI literature and explained in the sections 2.1 and 2.2 (pp. 2024 ), which acts as an employee OI marker in business organizations.

Previous research showed that employees' perception of the status and identity of the organization influences employee organizational identification. That is, when an organization is perceived to be more attractive, employees are more likely to develop 
stronger identification. The construed organizational attractiveness is sometimes contextualised as synonymous with the emerging corporate social responsibility agenda of organizations (Rodrigo \& Arenas, 2008; Rupp, et al. 2006; Vlachos, Theotokis, \& Panagopoulos, 2010) Therefore, this impacts on employees' organizational identification since corporate social responsibility and ethical practices debates have in recent times occupied a central position in most organizational strategic management practices.

However, despite the growing research on how corporate social responsibility and ethics affect employee attitudes and behaviours, especially relative to employee organizational identification, this is still an under researched area (Brammer, Millington, \& Rayton, 2007; Hansen et al., 2011; Humphreys \& Brown, 2008; Peterson, 2004; Preuss, Haunschild, \& Matten, 2009). Thus, it can be deemed as an area of organizational identification where future research work can be conducted, especially regarding the role identification may play in how corporate social responsibility affects employees' attitudes and behaviours in the workplace.

Another identity component worthy of mentioning in the attempt to map the precursors of $\mathrm{Ol}$ in organizations is the corporate culture perspective, which is of particular relevance, as argued by Podnar, Golob and Jancic (2011), because there generally seems to be a misconception of lumping all forms of identification (corporate culture inclusive) in organization behavioural theory studies under the umbrella term of "organizational identification". Extant corporate marketing studies emphasise their differences.

One such study distinguishes corporate identification as different from that of members of an organization who typically affirm their identification by highlighting their shared commonalities or attributes with other members but more reflective of members who identify with the observed culture of the company or corporation itself and focused on the organization's perceived image or branding (Balmer, 2008; see section 2.2, pp. 22-24). Likewise, Siegel and Sisaye (1997) argued that OI is a coherent function of both an organization's corporate identity and an employee's selfidentity. 
Explaining this further, Bligh and Carsten 2005 (cited in Podnar, Golob \& Jancic, 2011) suggested that individuals often view and construct their membership of organizations to which they belong in a dual manner. Firstly, by forming a cognitive bond with the organization as an entity and secondly exhibiting corporate identification traits by absorbing and enduring favourable images of such organizations which can also be mediated by clear and genuine communication and explanation of the organization as a brand to such organizations' members, that is, the employees.

Interestingly, though most OI studies typically assume that undertaking these actions often results in positive identification with the organizations, other scholars have argued that it could also have a counter-productive effect. For example, Pratt (1998) argues that some employees may actually want to retain an independent sense of self, thus they draw a distinction between their own self-identity and that of the organization. If this scenario occurs it will often result in the employee developing non-identification that is disidentification status according to Elsbach and Bhattacharya (2001) instead of identification.

Thus, perceived external prestige, often construed in terms of corporate identity or image and organizational culture of the company, can be said to be a significant element that determines how and in what form an employee develops identification within an organization. That is, existing literatures identify an organization's corporate culture as an OI Marker. This is mediated by the clarity and genuineness of communication from such organizations to the employee(s) in question.

Explaining the relevance of organizational culture in more detail, a substantive academic body of research has examined the nature and popularity of the organizational culture concept (Hofstede et al., 1990; Lindbo \& Shultz, 1998; Rousseau, 1990; Schein, 1990; Witmer, 1997). Other scholars recognise the relative existence of one form of culture or the other in organizations, despite the varying breadth of meaning it relatively connotes (Frost et al., 1991; Martin, 1992; Meyerson \& Martin, 1987; Schein, 1990). 
Organizational culture is said to be learnt by individuals in a group over a period of time, and such learning is simultaneously a cognitive, behavioural and affective process (Schein, 1990). Organizational culture is also said to be made up of both formal and informal norms that define employee beliefs, attitudes and conduct (Kerr \& Slocum, 1987).

Previous studies have linked organizational culture to observable organizational behaviours members' exhibit. Examples include newcomers' socialisation experiences within the organization as well as shared ideological norms increasing identification behaviours exhibited among employees. Specific research by Gibson and Papa (2000) concluded that because newcomers often strongly identify with the goals, values and objectives of the organization, they are indirectly subject to organizational control mechanisms which can be either concertive, or unobtrusive in nature. However, Glaser, Zamanou and Hacker, in their 1987 study, argued that for researchers to affirmatively answer organizational culture questions as to whether as a concept it can be managed or measured, it is necessary to have a definitive and agreed definition of what organizational culture is.

Other outcomes of organizational culture include the relationship with employee retention (Sheridan, 1992), person-organization fit (O'Reilly, Chatman, \& Caldwell, 1991), productivity (Kopelman, Brief, \& Guzzo, 1990), decision making (Gamble \& Gibson, 1999); thus signposting that organizational culture is ubiquitous.

Finally, as noted earlier in the chapter, Tompkins and Cheney (1985) suggested that if concertive control is developed and maintained through the process of identification, organizations may be able to strategically improve their overall performance and productivity. Thereby, an 'idealistic culture' develops which can then become the primary mechanism through which the organization exercises unobtrusive control. That is, the organization attempts at indirectly influencing the $\mathrm{Ol}$ process and behaviour of its employees, via the set beliefs, values and information communicated through an organization's culture (Bullis \& Tompkins, 1989).

Hence, organizational culture could be a strong predictor of employee $\mathrm{OI}$ in business organizations. 


\subsubsection{Organizational Identification and Communication}

Communication within the organization is said to be a strong predictor of organizational identification within companies (Bartels et al., 2010). This relationship between intra-organizational communication and identification has been described in a variety of ways. These include: the hierarchical position of the sender in relation to the recipient; direction of communication; level of abstraction; the communication function; content; and the form of the communication (Bartels et al., 2010; Dennis, 1974; Postmes, 2003; Redding, 1973; Smidts, Pruyn \& van Riel., 2001).

In relation to the content and form of intra-organizational communication, there are several frequently used dimensions of the communication climate, including the adequacy of the information being transferred, transparency and genuineness, participation in decision making and top management support (Guzley, 1992; Putti et al., 1990; Trombetta \& Rogers, 1988; Wallace, 1995). According to Bartels et al. (2010, p. 212), a communication climate is "...the collective communication components of the work environment, such as the perceived accessibility of management to employees and the reliability of information circulated within the organization".

Dennis (1974, cited in Bartels et al., 2010) also defined this climate as "a subjective experienced quality of the internal environment of an organization: the concept embraces a general cluster of inferred predispositions, identifiable through reports of members' perceptions of messages and message-related events occurring in the organization". This communication climate positively relates to identification at various levels of an organization. It has also been found to have a positive connection with organizational identification (Bartels et al., 2006; Smidts et al., 2001).

Relative to communication direction, communication climate is defined based on two intra-organizational hierarchical directions: vertical and horizontal communication (Bartels et al.,2010; Postmes et al., 2001). Defining both types of communication in relation to identification within organizations, Bartels et al. (2010) said that vertical communication is that which travels up and down the hierarchy, while horizontal communication refers to that between colleagues on an equal hierarchical level. An example of vertical communication would be between top management and the work 
floor and vice versa, while horizontal communication would be between two colleagues on the same paygrade level within a business.

Vertical and horizontal communication are said to have different impacts on higherorder and lower-order identities. Higher-order identity is associated with vertical communication, by increasing employees' identification with a larger organization or an entire organization (Ashforth \& Mael, 1989). However, lower-order identity is associated with horizontal communication, which relates to employees' identification with groups, such as specific company units and increasing attachment and cohesion within such groups (Levine \& Moreland, 1990).Nonetheless, Postmes et al. (2001) argued that both vertical and horizontal communication are positively linked to organizational commitment. Bartels et al. (2007) also emphasised that identification with a specific organizational level (work group, department) could be predicted best from the perception of its apparent communication climate, on that organizational level.

The Bartels et al. (2010) study indicates that vertical communication is a stronger predictor than horizontal communication of commitment which is likened to organizational identification. Vertical communication helps to define an organization's identity, that is, to convey what the company stands for (Dutton et al., 1994). Examples of such vertical communication include information about an organization's strategy or objectives and updating employees of organizational activities and developments which could help the employees in determining their position or fit within the organization. These could be in terms top-down or bottom-up communication (Downs \& Adrian, 2004). Top-down examples include such as "adequate information provision", "support of top management" and "reliability of top management" or "bottom-up communication sent from work floor to management level such as employees' opportunities to participate in decision-making..." (Bartels et al., 2010).

Benefits of vertical communication include the ability to minimise uncertainties about the organization's bearings to better employees' understanding of what the company stands for. This is fostered by clear information on the organization's set mission, goals and achievements which enables employees to identify organization 
characteristics (Dutton et al., 1994). Another advantage is that it enables the organization to inform its employees about the company's unique attributes which distinguish it from its competitors (Ashforth \& Mael, 1989; Cheney, 1983; Postmes et al., 2001; Postmes, 2003), hence creating a climate that fosters employees' organizational identification.

Horizontal communication, on the other hand, is often task-related and informal (Postmes, 2003). Task-related communication involves exchange of tasks information, such as for fine-tuning of in-company activities, while informal communication may entail non-work-related conversations between employees, both of which may influence employees' sense of identification and belongingness with the organization (Postmes et al., 2001). Thus, vertical and horizontal communication can be said to be strong predictors or markers of $\mathrm{OI}$ in organizations

\subsubsection{Organizational Identification, Organizational Citizenship Behaviours and Employee Unlearning}

According to Katz (cited in Humphrey, 2012), there are three essential behavioural types required for organizations to function effectively. The first stipulates that 'people had to be persuaded to join and remain with the organization'. Secondly, the people 'had to dependably perform their assigned tasks' and lastly, there should be 'innovative and spontaneous behavior' in achieving work objectives that exceeds the confines of their formal job role demands. Bateman and Organ (1983, cited in Humphrey, 2012) termed such spontaneous acts "citizenship" behaviours and from there Organizational citizenship behaviour research developed, mostly within the marketing and service literature.

Previously defined as "individual behavior that is discretionary, not directly or explicitly recognized by the formal reward system, and that in the aggregate promotes the effective functioning of the organization" (Organ, 1988, p. 4, cited in Humphrey, 2012), organizational citizenship behaviour is not a mandatory requirement for organizational employees, neither are they rewarded, rather the individuals choose to perform such acts on their own. 
More recently however, organizational citizenship behaviours have been linked as antecedents of organizational identification in organizations. Thus, a more current definition evolved as the exhibition of seven (7) common dimensions: helping behaviour, sportsmanship, organizational loyalty, organizational compliance, individual initiative, civic virtue and self-development' (Podsakoff et al., 2000). These elements collectively help define the understanding of employee unlearning in defining organizational identification in the literature and some evidential research also ascertains the causal relationship between both terms mediated by transformational leadership styles (Humphrey, 2012). Employee unlearning is primarily the understanding that for employees to be open to form a bond with their current organizations, they would go through a process of unlearning past ideas or experiences, and form new ones. Only then can they be effective in attaining good and positive performances as well as outcomes. Thus, the process of employee unlearning has become a strong indicator of employee identification in organizations.

\subsubsection{Organizational Identification, Power Structures, Organizational Leadership and Motivation}

Fairly recent studies on organizational identification have recorded outcomes evidencing the influence of '...dynamic, interactional, and interpersonal factors in enhancing employee organizational identification, such as including leadership factors...' Leaders have been said to be able to shape their followers' identities (Ellemers, De Gilder, \& Haslam, 2004; Avolio, Walumbwa, \& Weber, 2009), as well as organizational identification. As employees' immediate leaders play an important role in their day to day work lives within an organization, these leaders' behaviours may shape how employees view their relationship with their work organization. That is, leadership behaviours acting as power structures can act as a motivational or demotivational factor for employees within an organization.

Despite the increasing number of studies examining the impact of leadership on employee OI, this body of literature is still fairly confined and has many unaddressed issues. For instance, there is no clarity provided as to how individual differences or situational factors may affect the impact of leadership on employee Ol. An attempt is however made to fairly address the question within this research. 
Also, employees with an intrinsic need for affiliation, such as those with a stronger need for Ol, according to Kreiner and Ashforth (2004) as well as Wiesenfeld et al. (2001), can develop stronger OI regardless of leadership behaviours. Similarly, those who are more receptive to social influence(s) such as agreeableness and submissiveness are likely to have their Ol development more easily influenced by power structures and general leadership practices within their organizations.

Within the SME context of this research, the need for the right leadership style(s) is particularly essential in small businesses as the outcomes of the right leadership often lead to sustained competitive advantage(s), encourage economic growth, expand social mobility as well as extending employment opportunities to individuals (Valdiserri \& Wilson 2010). These outcomes resonate with the perceived roles of leaders in small businesses in setting their organizational vision and mission as well as co-ordinating employees' activities towards the fulfilment of set organizational goals (Chowdhary \& Saraswat, 2004). Without robust leadership, small businesses would have trouble surviving in highly competitive markets and could eventually close down or their growth might be severely impeded.

Furthermore, effective leadership skill is said to be essential in facilitating employees' ability to improve organizational successes through improved profitability (Appelbaum \& Valero, 2007). In line with this notion, Bradley and Cowdery (2004) also stated that in respect to small businesses, the leader is fully responsible for the organization's successes or failures. In support of the above, Beaver (2003) also noted that poor or ineffective management, leadership, capitalisation, business planning, industry knowledge and lack of a realistic vision, all of which are responsibilities of leaders or managers in organizations, often lead to business failures. However, guided by a clearly defined vision provided by leaders, employees would often commit to that vision and consequently contribute to the achievement of overall organizational goals, objectives, productivity and profitability.

Furthermore, Gordon and Yukl (2004) advocate for more research on leadership skills, especially relevant within turbulence-ridden and unstable small business environments. As regards facilitating achievement of organizational goals and objectives, small business leadership must take the reins of the organization securely 
and facilitate development of employee trust and confidence. This would often also mean more longevity for the business.

The relationships between certain leadership styles, profitability and organizational successes have also been explored in literature. Thus, providing leaders, especially in small businesses, with the wealth of knowledge required to become robust leaders, as well as how a better understanding of such leadership styles could affect organizational productivity and successes.

For instance, employee organizational identification has been found to be positively related to various leadership styles, including transformational leadership (Liu, Zhu, \& Yang, 2010; Carmeli et al., 2011) and ethical leadership (Walumbwa et al., 2011). According to OI literature, however, the impact of leadership on OI, may be conditional. For instance, Epitropaki and Martin (2005) suggest that transformational leadership's positive impact on employee OI could depend on employees' affective experience(s) within an organization. Meanwhile, evidence also suggests that the impact of transformational leadership on employee $\mathrm{Ol}$ is often stronger for employees with more frequent negative affective experience(s) or less frequent positive affective experience(s) within an organization (Epitropaki \& Martin, 2005).

Also, power structures in organizations as characterised by the leadership are often a function of elements such as personalised consideration (attending employees personalised needs); intellectual stimulation (conveying the significance of job meanings to employees, encouraging them to challenge their assumptions and limitations to take risks and consulting with followers' before making decisions); inspirational motivation (simply put, inspiring the best from the employees to achieve set goals); and creating an influence relationship (that is, acting as role model(s), standardised ethical practices and helping employees develop a sense of self-worth and confidence) (Bass \& Avolio, 1994).

This situation is more commonly known as leader-member exchange based on social exchange theory (Blau, 1964) that promotes that the higher the relationship quality between managers and their employees in organizations, the more likely the employee is to feel obliged to the manager and thus the organization (Coyle-Shapiro 
\& Conway, 2004; Gouldner, 1960, both cited in Farrell \& Oczkowski, 2012). This is because managers of small businesses are also often the owners of the business, with a dual owner-manager role. Thus, employees in positive exchange relationships with their owner-managers often feel more valued by the organization, and a part of its 'most valuable group' and vice-versa, whereas a recent study suggests that employees with weaker levels of leader-member exchange levels tend to consequently identify weakly with their organization (Tangirala et al., 2007).

Transformational leadership is also said to be potent in influencing employees' behaviour and performances in organizations, as it positively enhances employees' organizational identification (Kark, et al., 2003; Liu, et al., 2010) as well as boosting job performance through employee identification with the work unit (Walumbwa et al., 2008). This is similar to Liu et al.'s (2010) assertion that transformational leadership can foster followers' voice-behaviour because it enhances their organizational identification.

Another form of leadership, known as ethical leadership, is also known to be a strong determinant of employees' behaviour in organizations. Consisting of two main dimensions: 'moral person' and 'moral manager', ethical leadership (EL) (Brown, Trevino \& Harrison, 2005; Brown \& Trevino, 2006; Brown \& Mitchell, 2010), on one hand, explains leadership attributes that demonstrate 'normatively appropriate conduct through personal actions and interpersonal relationships' and promote 'such conduct to followers through two-way communication, reinforcement, and decision making' (Brown et al., 2005, p. 120). The 'moral person' dimension voices that ethical leaders have strong moral characteristics and traits, such as honesty, trustworthiness, approachableness, care and fairness, while the 'moral manager' dimension suggests that ethical leaders set and communicate ethical standards to their subordinates and invariably enforce those standards.

According to Walumbwa, et al. (2011), ethical leadership behaviours are most likely able to promote follower(employee) organizational identification because ethical leaders often succeed in improving employee cooperation, organizational trust, feelings of mutual respect and self-esteem within an organization. This is also related to the assertion that employee organizational identification serves as an important 
psychological mechanism in exploring the relevance of ethical leadership on employee task performance (Walumbwa, et al., 2011). A criticism of these studies however is that most research exploring the influence of leadership styles on employee $\mathrm{OI}$ is still being conducted with reference to limited styles of leadership, especially transformational leadership (He \& Brown, 2013).

\subsubsection{Organizational Identification, Intra-organizational Interactions and Job Satisfaction}

Another antecedent of organizational identification is job satisfaction (Berger et al., 2006; van Dick et al., 2004). Hackman and Oldham's (1980) generalistic view referred to job satisfaction as the degree or extent to which an employee experiences happiness in his or her job. This is similar to the Locke (1969, p. 316, cited in He \& Brown, 2013) definition as "the pleasurable emotional state resulting from the appraisal of one's job as achieving or facilitating the achievement of one's job values".

However, this state of happiness or job satisfaction is often a result of a combination of different factors including job remuneration, relationships with colleagues and superiors at work, the type of work an employee is engaged in as well as the autonomy or independence an employee enjoys in their work practices within the organization. Brief (1998) categorised these component elements into two main categories, namely objective job circumstances and individuals' interpretations. This notion thus also contributes relevantly to the development of the conceptual research framework and the division of the Ol markers into the two main identified categories.

Objective job circumstances are situations in which the employees find themselves, but over which they do not have jurisdictive control, while the individualised interpretations category reflects their individualised belief system(s) and perspective(s) relative to organizational activities implemented in their relevant organizations. These are known as employer situational-context markers and employee situational-gap markers categories respectively.

For example, within the employee situational-gap markers category, employees in organizations can develop their $\mathrm{Ol}$ statuses by constructing how they perceive the 
organizations they work in through activities or practices such as satisfaction with their relationships with other employees (Alvesson, 2000). That is, how potent and satisfying are their intra-organizational interactions, which also indirectly reiterates the importance of an employee's identification with the organization's corporate culture (Balmer, 2008). This is termed as group identification.

Due to the differences within these Ol marker categories, employees may experience variant feelings over time. That is, an employee may be fairly satisfied with certain aspects of their job and simultaneously not with other aspects; thus, following this analogy, job satisfaction could be understood through the aggregate lenses of all the different aspects of their job. However, most literatures regard the concept of job satisfaction as a generalisable overarching attitude an employee displays towards their job. This suggests that employees that identify themselves highly with their organizations often tend to be considerably more self-confident about being a member of the organization, and their respective job roles within the organization and vice-versa. That is, they often have a strong feeling or sense of self-worth even in unfavourable circumstances such as low pay or reduction in salaries. This may also be attributed to their perception that such unfavourable occurrences are necessary for implementing and achieving set organizational goals and objectives.

In support of the above argument, highly identified employees would often see their respective job elements in a positive light when they feel these characteristics are consistent with the set organizational goals and identity portrayed. Causatively, if employees identify themselves with organizational attributes they consider favourable attributes; they will feel mostly satisfied and motivated in their respective job roles (Harris \& de Chernatony, 2001; Wheeler et al., 2006).

\subsubsection{Organizational Identification, Employee Performance Management and Organizational Practices}

In addition to the earlier discussed markers of employee organizational identification in businesses, some other organizational practices and strategic activities often employed by management to improve their overall organizational performance have also been highlighted in OI literature. These activities are often tailored to meet the developmental needs of the employees in such organizations and are discussed 
within this sub-section. Firth et al. (2004) also stated that an associated benefit of implementing and encouraging these practices is the advantage of minimising the cost of recruitment, training and cost of efforts invested in retraining new employees.

One of such organizational practices is Job Crafting, which is an employee behavioural concept regarded as positively and directly impacting on employee performances on the job (Berg et al., 2010; Wrzesniewski \& Dutton, 2001). Job crafting as a behavioural change construct, when well-planned and implemented, is said to often increase employee skills, motivation and integration of personalised goals with the overall job goals (Berg et al., 2010; Wrzesniewski \& Dutton, 2001). These highlighted changes can then result in better work efficiency by ensuring set performance indicators are met as well as an increase in on-the-job motivation (Tims, et al., 2012; Bacaksiz, Tuna \& Seren, 2017). Also, linked closely to increasing job satisfaction, motivation and performance, job crafting reduces work stress, making work-life for employees more meaningful and generally facilitating a healthier working environment (Berg et al., 2013; Grant, 2007; Rosso et al., 2010).

Other advantages job crafting creates, according to Crum and Langer (2006), include encouraging positive changes in employees' thoughts, relations and contextually all work is. Generally, it therefore creates positive behavioural shifts in employees' attitudes to their work and thus helps maintain a weighted and balanced viewpoint between management expectations about a given job role and employees' individual desires, without much hindrance from their immediate working environment and availability of resources (Tims \& Bakker, 2010). This job crafting attribute is often expressed as an element of an employee's organizational identification process, as a form of job creativity, since crafting enables employees to practically express their work perceptions as well as focus on satisfying elements of their job roles, rather than engaging in a myriad of duties that may not necessarily be satisfying (Oettingen et al., 2001).

Employee turnover issues can often be a major source of worry in organizations, especially in small businesses in which the source of their competitive advantage is often a function of and reliant on their employees' performance and expertise (Santhanam, Kamalanabhan \& Dyaram, 2014). Hence businesses tend to engage in 
organizational activities and practices focused on keeping the turnover level as low as possible. As a proxy for an actual employee turnover index, employee turnover intention(s) is often observed as behaviours or attitudes at work. Thus, the Ol status of employees within the organization is often a precursor of observed behaviours or attitudes in relation to their intent to leave or not to leave the company (Ashforth \& Mael, 1989; Santhanam, Kamalanabhan \& Dyaram, 2014).

According to Santhanam, Kamalanabhan and Dyaram (2014), organizational identification plays a mediating role between human resource management and developmental practices such as recruitment, selection, training, career growth opportunities, performance appraisal and compensation and employee turnover intention. They suggested that if and when managers carefully design, plan and implement these human resource management and developmental practices, facilitated by employees' organizational identification status, positive outcomes such as reduced turnover rates or intention to leave the business and improved performances tend to occur.

\subsection{Filling the Organizational Identification Literature Gap}

There are evidently diverse uses for organizational identification research in business management. These include usage in situations of change management in organizations, such as structural changes like mergers and acquisitions. This is because individuals might fear a threat to the stability and identity of the company when a merger occurs. Another potential use would be in situations when organizations are continuously revamping their psychological contract with their employees to stay afloat in today's unsettling and continuously changing business economic climate.

Despite the various studies on $\mathrm{OI}$ related to such as commitment, job satisfaction and employee retention, there still remains limited evidence to show the actual extent to which training activities influence Ol statuses within organizations and more specifically small businesses. Researchers have not clearly established the relationship between employee training and OI, in particular, whether or not employee training has any significant impact either directly or indirectly as an identity regulator within an organization. 
Empirical evidence to date is mostly based on findings supporting the notion that high level of organizational identification has a direct positive correlation to increased organizational commitment, job satisfaction and higher employee retention figures (Frone \& Finn, 2004; Riketta, 2005; Carmeli, Gilat \& Waldman., 2007; Walumbwa et al., 2008). Thus, exploring the dynamics of employee Ol levels relative to perception of training programmes participated in within small businesses will help this research contribute considerably to the extant literature on the OI subject being studied.

\subsubsection{Organizational Identification and Human Resource Management Practices.}

Organizations engage in various HRM practices to equip their employees with the required knowledge, skills and attitudes. When these practices lead to improvements that are predominant in an employee population, this will be sufficient to influence organizational performance (Bowen \& Ostroff, 2004; Kozlowski \& Klein, 2000)

One such HRM practice is training which is a tool widely known and used in organizational practice and discussed in research literatures (Waterson et al., 1999; Wood et al., 2004). Employee training enhances employee knowledge specific to an organization which can then be applied to their day to day job roles in the workplace (Appelbaum et al., 2000; Pfeffer, 1994; Way, 2002).

Linking the significance of training as an HRM practice to the employee organizational identification process is a primary aim of this research. This is mostly apt in contemporary management practices, as HR activities seem to have grown attentive to employees' activities of defining self-identity; for example, Alvesson and Willmott (2002) suggest that regulating the self-identity of employees through HRM activities including wellbeing management, developmental trainings and team building practices can serve as an internal and more effective control compared to external stimuli which insufficiently control the inside "hopes, fears, and aspirations" of employees.

The argument about self-identify in workplaces is also elaborated in the perspective of multiple self-identities (Scott \& Lane, 2000; George \& Chattopadhyay, 2005; Hsu \& Hannan, 2005; Ashforth et al., 2008). For example, a person performing different roles for different categories of people within and outside the organization, such as a 
husband who is a sub-ordinate and manager within an organization, will often exhibit different identities for the different roles and these various self-identities may conflict with each other.

Employees may need to integrate these multiple and possibly ambivalent identities into a united and functional unit in order to be more productive by avoiding conflicting identities. The integration of these multiple identities is often referred to as organizational identification that has been consistently suggested as a positive job attitude (Ashforth et al., 2008), especially when the consensus occurs between an employee and the collective self.

Another relevance of $\mathrm{HR}$ practices is the assertion that HR management and developmental practices such as organizational training interventions when employed in organizations can have a number of positive outcomes such as improved employee skill(s) and performance(s), thus, resultantly improving an organization's holistic performance (Santhanam, Kamalanabhan \& Dyaram, 2014). Organizational identification plays a mediating role between human resource management and developmental practices such as training.

\subsubsection{Understanding Employee Training in Learning Organizations}

According to Noe (1999), "training refers to a planned effort by a company to facilitate employees learning of job-related competencies". This includes the knowledge and skills that are essential for successful job performance. The aim of training is for employees to master the knowledge and skills emphasised in training programmes and to apply them to their daily job roles.

Employee training as a topic has many facets. Typically, two major types of training exist: off the job and on the job training. Off the job training is usually carried out in locations outside the workplace and is often associated with high cost of implementation because it is usually contracted out to professional consulting firms (Hackett, 1997).

On the job training, on the other hand, is training that involves acquisition of job skills and is conducted within the workplace (Hackett, 1997). This has its own disadvantages such as finding the right time to conduct it and ability to monitor and 
evaluate its effectiveness. Despite these negative aspects, however, it possesses some unique advantages which include: cost effectiveness; access to immediate feedback for evaluation purposes within business organizations; continuous productivity of employees while undergoing training as no time is lost on the job; and employees are actually learning by doing (Jacobs \& Jones, 1995).

This research focuses on exploring the significance of both types of training activities on Ol processes of SME employees, as despite their respective drawbacks, they both have the potential to improve significantly the performance of employees within organizations which summatively adds up to overall organizational performance.

Almost all employees undergo one form of training or another during their careers. It has been identified as a key HRM practice that aids and contributes to gain organizational competitive advantage (Schuler \& MacMillan, 1984). Employee training is often expected to improve employee knowledge specific to the organization and this can then be practically applied to their day to day job roles in the workplace (Appelbaum et al., 2000; Pfeffer, 1994; Way, 2002).

According to Scullion and Collings (2006), employee on the job training and development is a crucial aspect of the human resource management unit of every organization, particularly as it affects the strategic positioning of business organizations in the highly competitive economy existing in the present day. Also, employee training is among the most widely known HRM practices in both research literature and organizational practices (Waterson et al., 1999; Wood et al., 2004). While some other HRM practices such as recruitment, employee remuneration and appraisal can play a role in affecting organizational performance (Birdi et al., 2008), in this study they are viewed and weighted to be of less significance to the subject matter.

Other definitions of training include viewing training as "a medium for developing human resources in business establishments, to motivate employees and improve their on the job performance" (Roy \& Raymond, 2008), which is linked to job satisfaction (Sahinidis \& Bouris, 2008). Training has also been identified as a key Human Resource Management (HRM) practice that aids and contributes to gain organizational competitive advantage (Schuler \& MacMillan, 1984). Employee 
training is also said to enhance employee knowledge specific to the organization and this can then be practically applied to their day to day job roles in the workplace (Appelbaum et al., 2000; Pfeffer, 1994; Way, 2002).

Thus, bolstering employees' job satisfaction through training is an important organizational lever. The HRM/D profession has however been scrutinised on the credibility of its contribution to organizational successes, thus developing the universalist and contingent schools of thought (Delery \& Doty 1996; Becker \& Huselid 1998). While the universalist approach hinges on "best practices", the contingent advocates "best fit" HRM/HRD practices (Huselid, 1995; Becker \& Huselid, 1998).

Some scholars have nevertheless argued that training does not necessarily exhibit the desired effect. Many organizations have good training plans, but these are often not specific about what the training will achieve. They use an "umbrella approach" rather than tailoring it to accomplish a specified goal. Hence training has been turned into a socialization tool and norm rather than an avenue to actually improve employee skill and knowledge (Barton \& Delbridge, 2001).

Furthermore, Morrow (2001) argued that the benefits of training are often not instantaneously perceptible and can be delayed, also that effective training is often costly and requires professional capability which is not easily come by.

It is essential, however, to note that despite these arguments training is still a universally acceptable tool used by business organizations to upgrade employee skills and gain new knowledge in a bid to improve the overall performance of employees and thus the organization (Mathieu et al., 1992). Nonetheless, this in itself is not a criterion to ascertain the effectiveness of training, especially in mitigating operational risk within an organization. Therefore, in ensuring that training meets its set goal, effective delivery methods and evaluation techniques need to be employed to analyze results and justify training expenses.

Organizational performance levels have been said to be influenced positively by implementing relevant HRM practices such as training. Becker and Huselid (2006) asserted that studying strategic HRM both as an essential element of managerial 
practice and from an academic perspective has gained considerable attention in the past 30 years. This understanding is based on the theoretical assumption developed by Barney (1998) about the relevance of the Resource Based View of the firm. Based on the idea that businesses' organizational performance can be improved to enable them to sustainably compete globally, the resource-based view rationale advocates for organizations to develop their strategic resources to gain a sustainable competitive advantage (Barney, 1998; Denton \& Denton, 1999; Power \& Waddell, 2004; and Senge, 1990).

In support of this claim, Lado and Wilson (1994) argue that HRM practices help with the development of specific knowledge and embedding it in an organization's culture and hence its employees, creating a potent and sustainable competitive advantage. Similarly, HRM practices are said to aid the development of employees' knowledge, skills as well as their attitudes and behaviours. These improved attributes when predominant in an employee population are said to have a significant positive influence on organizational performance (Bowen \& Ostroff, 2004; Kozlowski \& Klein, 2000). Such influential HRM practices include training, recruitment, employee remuneration and appraisals (Birdi et al., 2008). According to Scullion and Collings (2006), moreover, employee training and development are of crucial importance in terms of their impact on the strategic positioning of such businesses in their often highly competitive market niche. Training is also generally a key and well-known function of HR (Wood et al., 2004).

Despite the apparent relevance of these strategic HRM practices to improving organizational performances, such interventions have been criticised for providing little or no specificity on the length of time duration required to experience or observe the benefits of their implementation in terms of improved performance. It is argued further that such undefined time-frames may invariably conceal some interventionoutcome interactions (Kozlowski \& Klein, 2000).

With respect to such HRM practices, this research focused on employee training (or learning as its sometimes interchangeably called) as the HRM intervention to be explored relative to the subject matter of employee organizational identification development within small businesses, as most employees undergo one form of 
training or another during their careers. Training has also been identified as a key HRM practice that enables firms to gain competitive advantage if employees apply the specific knowledge learnt in their day to day job duties in the workplace (Appelbaum et al., 2000; Pfeffer, 1994; Way, 2002). A primary aim of training in organizations is often to help employees to master the knowledge and skills they currently possess, gained from training attended, or those they hope to develop. It is when the knowledge gained is applied to their daily job roles that it is impactful. Roy and Raymond (2008) went on further to iterate that training is a medium for developing and motivating employees in organizations, thus, ensuring positive job performance(s).

Some scholars have however questioned the predictability of training outcomes, arguing that outcomes can be unpredictable, such as in cases where organizations have good training plans but with no assurances of the outcomes expected. Thus, they may often use an "umbrella approach" rather than tailoring training to accomplish set goals (Adekunle, 2011), which suggests that training has been turned into socialisation tool rather than a developmental tool to help improve employee skill and knowledge (Barton \& Delbridge, 2001). Also, training benefits are often not instantaneous, can be delayed, costly and sometimes require professional capability which is not easily come (Morrow, 2001), especially by small businesses.

According to lqbal et al. (2013), few studies have explored the relationship between employees' expectations and those of their employers after implementing training interventions as a mediating factor or considered possible consequences that could arise when the expectations of both parties are not met. Tensions may also arise from time to time in an attempt to fulfil the psychological contracts between them. Also, changing environmental factors (often out of the control of employees and employers) may impact on the achievement of these expectations, as uncertainties may arise from the market. These changes may consequently affect the employee and employer behaviour in the context of contractual relations (Top, 2013).

With employers' expectations often evidently recognised through observable performance improvement and productivity acting as a return on training investments, employees often tend to be a major source of competitive advantage for 
businesses, especially smaller companies (Yamnill \& McLean, 2001). There is powerful agreement that while acquisition of information, abilities, behaviours, and mind-set occurs through training, training is of little worth if the new characteristics are not generalised to the job setting and are not sustained over time (Kozlowski \& Salas, 1997). Hence if no noticeable organizational dividend is observable, training is deemed to be ineffective (Yamnill \& McLean, 2001). According to Swanson (1995), for human resource development to become a vital organizational function, performance is the key. Therefore, if organizations understand that training really creates positive differences in organizational and employee efficiency, they should also realise how to support and implement training within their organization.

Additionally, organizations often spend a lot of financial funds on training their employees to meet their organizational objectives, as employers believe training has a strong correlation with superior performance. Thus, employers often have huge expectations from their employees post-training, to compensate for the amounts spent to train them. Moreover, Kuchinke (1995) argued that training is not just an organizational tool but can also be an internal behaviour result. Thus, both employees' and employers' expectations are important when considering training efficacy in businesses.

In terms of expectations, lqbal et al (2013) asserted that employers tend to emphasise the impact of training on performance rather than as a career developmental tool for employees, which again causes major dissonance between employer-employee post training expectations. However, if handled strategically, training can be a motivational tool to contribute to improved organizational performance. Employees may then see career expansion opportunities as deriving from such as career development programmes, and effective training can also have a positive impact on employee retention figures (Kim, 2010).

Despite the contrasting arguments on training, as an HRM tool it is still a universally accepted and used by all types of businesses (including small businesses) to improve their organizational performance by directly improving their employee performance (Mathieu et al., 1992). However, it cannot be assumed that this criterion 
alone is sufficient to ensure that training will impact on all organizational behavioural concepts such as the OI topic being studied in this research.

Organizations engaging in training activities are often termed learning organizations. Senge's (1990) model of a learning organization is a key work in the understanding of what a learning organization is, signposting to the significance of learning disciplines; small business sustainability; and the role of small business leadership in creating the sustainable learning culture in organizations.

The learning disciplines proposed by Senge (1990) include systems thinking which emphasises the interdependency of the various disciplines and encourages organizational members to view the entire system as a holistic unit. That is, employees see the relatedness they all share within their organizations. The second discipline, known as personal mastery, explores the organizational members' responsibilities to deepen and clarify their own personal vision, focus their energies, develop patience as well as try to see reality objectively, so as to achieve personal fulfilment.

The third principle, mental models, relates to the rooted beliefs, assumptions and generalisation of values that influence how learning organizational employees understand the world and take action (Senge, 1990). Explaining this further, a mental model is said to help us understand by critically reflecting on our knowledge of things. Critical reflection emphasises the need to makes sense of things by questioning situations and information rather than accepting them at face value (Brookfield, 2000 and Mezirow, 1997). The fourth principle is building shared vision. This encourages the creation of a genuine vision that members want to identify with and commit to, aided by "Learningful" conversations. A final principle is team learning which is said to begin with dialogue where team members partake in an authentic "thinking together" by carefully sharing with each other their personal assumptions as a team.

Thus, since the subject of $\mathrm{Ol}$ under scrutiny in this research is a psychological construct, Senge's mental model ideology is borrowed to understand what $\mathrm{Ol}$ is as a concept within small businesses, as learning organizations in their own right. To 
understand this, the research employed a combination of sense-making theories that is discussed further in the section 2.11 (pp. 72-78).

\subsubsection{Small and Medium-Sized Enterprises and Business Studies}

For the purpose of this research, the definition of SMEs is derived from a combination of both the DBIS (2015) and European Union (EU) 2003 definitions of an SME as "an entity engaged in an economic activity, irrespective of its legal form...enterprises which employ fewer than 250 persons; have an annual turnover not exceeding EUR 50 million and/or an annual balance sheet total not exceeding EUR 43 million". The choice of this definition is based on its specific nature and its applicability to the chosen study sample and UK statistics.

SMEs, or small businesses as they are sometimes called, are vastly numerous and operate in diverse economic sectors (Valadez, 2011), thus leading to controversy regarding a generalised definition (Fox, 2013). Nevertheless, SMEs often have some characteristics in common which have over time been used as criteria to differentiate them. These characteristics include: smaller employee numerical strength, lower financial turnover, lower assets, younger firm age and less complex management structures relative to their larger business counterparts (Lee \& McGuiggan, 2008).

Generally, business studies have highlighted SMEs to demonstrate various positive organizational attributes (David, 2013). Such attributes include employment creation. Prior to publication of the Bolton report in 1971, small businesses were not considered economically significant sites for detailed research studies. Curran and Blackburn (2001) stated that the prolific and complex people interaction attributes within small businesses made it quite complicated and almost impossible to quantitatively analyse data from these businesses, thus limiting the available research methodology to qualitative- based techniques and attracting little research funding. Post report publication, however, research interest in SME began to blossom, with Birch's (1979) study being a forerunner.

Furthermore, towards the end of the twentieth century, national economies of the world, including the UK, began to praise the role of SMEs for their non-substitutable activities in boosting financial earnings of economies and contributing significantly to national GDP growth through related job creation. Atkinson \& Storey (1994) add that 
UK government policy toward the end of the twentieth century was heavily influenced by the perception that small firms could contribute to alleviating the growing unemployment problem and wider economic issues by creating employment. Also, Valadez (2011) emphasised the economic significance of choosing SMEs (which in the UK could have turnover as high as $£ 50$ million) as a study niche by highlighting the relevance of their aggregate population density and financial contribution in sustaining economies on a cumulate scale globally relative to their larger businesses counterpart.

Nevertheless, in 1994, Storey cautiously argued that the effect of small businesses on employment growth in national economies may be inflated, as while SMEs contribute significantly and consistently to net job creation, larger firms also contribute significantly to employment figures during certain periods of rapid growth and expansion. A DBEIS (2017) statistical survey data release however substantiates Atkinson and Storey's (1994) assertion, with small businesses estimated to employ about $60 \%$ of all private sector employment in the UK and having a combined turnover of $£ 1.9$ trillion, accounting for $51 \%$ of all private sector turnover.

Another benefit of smaller businesses highlighted by organizational studies is their relatively small employee numbers, defined by DBEIS (2015) as between 1 and 249, making them more flexible and able to rapidly respond to changing business conditions as well as to meet the changing needs of employees and customer demands at short notice. Atkinson and Meager (1994) also substantiated the importance of company size in their study, suggesting that firm size is a great influencer of most employment decisions. This flexibility attribute can contribute to better profitability and growth in these small companies. For example, small businesses in the finance sector are likely to experience rapid growth where formalised systems such as computerised accounts and marketing plans are used to augment their employees' skills and expertise (Foreman-Peck et al., 2006)

Other interesting attributes of small businesses in organizational studies include: the peculiarity of family businesses; owner-managers' influence on control-systems within such businesses; as well as impact of age on SME organizational activities 
(Foreman-Peck et al., 2006; David, 2013). For instance, it is suggested that small businesses with older management are less likely to grow or their growth may be limited as they are often less risk-loving and are more likely to sacrifice future profits for certainty of the business's security.

The attributes discussed above, in combination with the researcher's own experience of working in SMEs (which originally inspired her research interest), make small businesses an interesting site for this Ol study.

\subsubsection{Benefits of Employee Training to Small and Medium-Sized Enterprises}

According to Scullion and Collings (2006), employee on the job training and development is a very important aspect of the human resource management unit of every organization, especially as it affects the strategic positioning of business organizations in the present-day competitive economy. Also, employee training is among the most widely known HRM practices in both research literature and organizational practices (Waterson et al., 1999; Wood et al., 2004).

Training programmes and activities have also been said to be a most effective mode of disseminating organizational values and goals simultaneously to employees (Shen \& Roger, 2006; Harzing, 2004 both cited in Jaiswal, Gupta \& Singh 2014). Exploring the benefit of training activities in organizations further, Helliriegel, Slocum and Woodman (2001) stated that increased organizational productivity through better job performance; more efficient use of human resources, continuously achieved targets, goals and objectives; prolonged reduced cost due to less labour turnover; reduced organizational errors, accidents and absenteeism rates; a more capable, and mobile workforce and; retention of existing staff members are all evidenced benefits of employee training of employees in organizations. This notion is also similar to Echard and Berge (2008 cited in Jaiswal et al. 2014) assertion that effective training techniques can produce significant business results especially in customer service; product development; new skill acquisition capability as well as the quality culture of a business, employee workforce, and ultimately the final product.

SMEs play a critical role in developing national economies by alleviating poverty, creating jobs and participating in the global economy to create economic growth and equity (Painter-Morland \& Dobie, 2009). Thus, to ensure continuity of their survival and sustainability, organizations have been challenged to learn faster and rapidly 
adapt to changes in their environment (Hattingh \& Smit, 2004), which means they need to be learning organizations. Indeed, their failure to survive would mean a collapse of most national economies globally.

Furthermore, Bressler (2012) stressed the importance of training for development of human capital assets/employees as a crucial factor for smaller businesses in maintaining sustainable competitive advantages, particularly in unstable economic climates. In addition to the above notion, both Shandratilek (1997) and Dessler 2005 (both cited in Jaiswal et al. 2014) emphasized that the inadequacy of expertise in an organization is a key constraint to having competitive advantage over others in the same industry, thus availability of high-quality employees with competent expertise can be facilitated through organising relevant training programmes and activities.

Additionally, small businesses often operate "on the ground", have closer links to their immediate communities and are often better positioned to effectively design tailored training strategies that address the needs of their relatively small numbers of employees.

Gratton et al. (1999) explored strategic positioning further as an alignment of set strategic objectives and people policies, proposing fit levels ranging from weak to strong in terms of gaining potential competitive advantage for the organization. Consequently, where a weak link is predominant, employees will not identify with organizational objectives or implicitly the business. To encourage employee identification, Albert, Ashforth and Dutton (2000) suggest that organizational identity and $\mathrm{OI}$ - that form the bedrock on which many observable organizational behaviours are built - need to be thoroughly understood.

Thus, this thesis explores how training, as a strategic HRM/D tool, influences the employee OI process within SMEs. Since, as an HRM/D tool, training has been linked to improvement of overall employee job satisfaction, as well as improved organizational performance (Sahinidis \& Bouris, 2008; Becker \& Huselid 1998), it can potentially be used as a strategic tool to influence the employee Ol process. This resonates with Gratton et al.'s (1999) view of the strategic fit of HRM/D practices such as training, as well as Alvesson and Wilmott's (2002) assertion on identity regulation work in organizations. 
However, despite recognition of the importance of training for any business, regardless of size and sector (Chaudron, 1996; Noe, 1999; Owens, 2006), much of the academic literature has specifically focused on multinational companies in suggesting that effective training as a strategic HRM tool improves organizational competitive advantage (Barney,1991). The rationale for this research focus on large businesses, as suggested by Valadez (2011), may be due to their relatively greater access to capital funds to fund such research projects, as well as the larger number of employees at their disposal to partake as study subjects.

Therefore, by virtue of their smaller numerical size and lower funding capacity, small businesses are at a disadvantage in terms of the options available to them to continuously develop their human asset base (Oriaku,2012; Fox, 2013). Also, according to the Department of Business Innovation and Skills (DBEIS), in 2017 an estimated total of 14.1 million people were employed by SMEs, accounting for $99.9 \%$ of all private sector businesses and $59.1 \%$ of private sector employment, with a combined turnover of $£ 1,500$ billion in the United Kingdom (UK).

The researcher also has experience of working in SMEs, which inspired her keen interest in situating the research within the SME context.

\subsection{Theoretical Models}

To further understand the potential influence of training as a regulatory tool on the dynamics of employee organizational identification processes in SMEs, it is pertinent to gain an insight into the conceptual and theoretical frameworks on which this study was based. Components within the conceptual research framework are explained in depth to elucidate their significance within the framework.

Thus, following on from the section 2.10.2 discussion on usage of mental model ideology (pp.61-67), to explain the exploratory concepts, this study employs sensemaking theories as the theoretical lenses through which the development of the conceptual research framework is designed. Sense-making is primarily concerned with the development of philosophical theorising in research studies (Savolainen, 2006), as well as metatheoretical sorting (Dervin, 1999). Its foremost concern is to 
make a set of assumptions and propositions of a theoretical nature, and to act as an encapsulation net that contains methods to make sense of or understand what people do in their daily experiences (Dervin, 1992).

\subsubsection{Understanding Sense-Making}

Gaining popularity in the 1970 s and 1980 s, sense-making was initially developed to study information needs, seeking and for communicative uses and thus defined as a communicative behaviour. However, with progress in studies Dervin (1983) noted that sense-making can actually be an observable behaviour which can have both cognitive and procedural elements (Internal and external representation respectively). Possession of these two components allows individual possessors to construct and design their own movement through the time-space context, which suggests that early studies on sense-making were skewed, primarily to cognitive and constructivist methods. Later studies from the 1990s, however, incorporated assumptions of communitarism in their sense-making ideologies (Dervin, 1994).

Every moment of sense-making is also said to involve some sort of energy-releasing components such as force, power, and constraint. Hence, these energies can come from individualised characteristics such as motivation, resistance or help received from others as well as other environmental conditions external to the individual (Savolainen, 2006). This creates a dichotomous casing, where humans seeking informational clarity are constrained by structural power forces (the organizational management in the context of this research) and individual principles which can sometimes challenge organizational norms and are thus seen as sites of power themselves. This dichotomous boundary comprises the employer situational-context OI markers and employee situational-gap OI markers respectively within the context this research study. And the function of sense-making is to effectively help navigate and position the understanding of the phenomenon under exploration in sections of best-fit within the identified dichotomous boundary.

Sense-making models are also based on an assumption that the understanding of an objective reality can be tempered by the observer or perceiver's sense-making process (Weick, 1993) and suggested that integration can only be achieved through a continuous and flexible negotiation between the self and the organization in context 
(Scott et al.,1998). Thus, both categorisations of power distribution in organizations have significant importance in understanding an organizational behaviour phenomenon.

Several sense-making theories have been postulated in literature and within different fields of academic study. However, this study adopts both Karl Weick's and Brenda Dervin's models to explore the research aim primarily rooted in employees' OI. The rationale for the choice of adopting both of these sense-making models is that each capture essentially different sense-making perspectives which when combined together give a much more holistic view regarding the process of sense-making and application within a business organization. Thus, the model facilitated the development and understanding of the respondents' stories and narratives to create meaningful assumptions.

While Weick's (1993) approach to sense-making focuses more on a situational awareness leading to normative organizational sense-making and tends to lay somewhat more emphasis on the significance of group work, Dervin's approach is more aligned with individual perceptions in relation to communication within organizations (Snowden 2008).

In its raw form, sense-making enables individuals to better grasp or understand the reality happening in their environment; in this context it is the organization itself which could be salient and influential in developing OI within that organization. Weick further stressed the importance of sense-making as the way we structure the unknown to be able to act on it, by meaningfully deducing plausible understanding from series of data collected, whether from actions, conversations or even retrospective thinking that makes us produce adaptive actions.

Weick's model is based on the notion that the construction of individual and organizational identities is an on-going sense-making process that can be impacted by specific organizational activities. Using the popular phrase "How can I know what I think until I see what I say?" the model identifies seven main components of the sensemaking process, namely: Identity Construction; Retrospection; Enactment; Socialisation; Continuation; Extracted Cues; and Plausibility. 
Weick's sense-making model as depicted in figure 1 below is however not devoid of limitations. Although it could help deepen the understanding of identity in workplaces, the model has not yet been fully embraced from the multiple-identity perspective, thus other scholars such as Park (2014) have explored this identity gap further; that is, whether there is a possibility that individual employees in organizations can have dual OI occurring simultaneously.

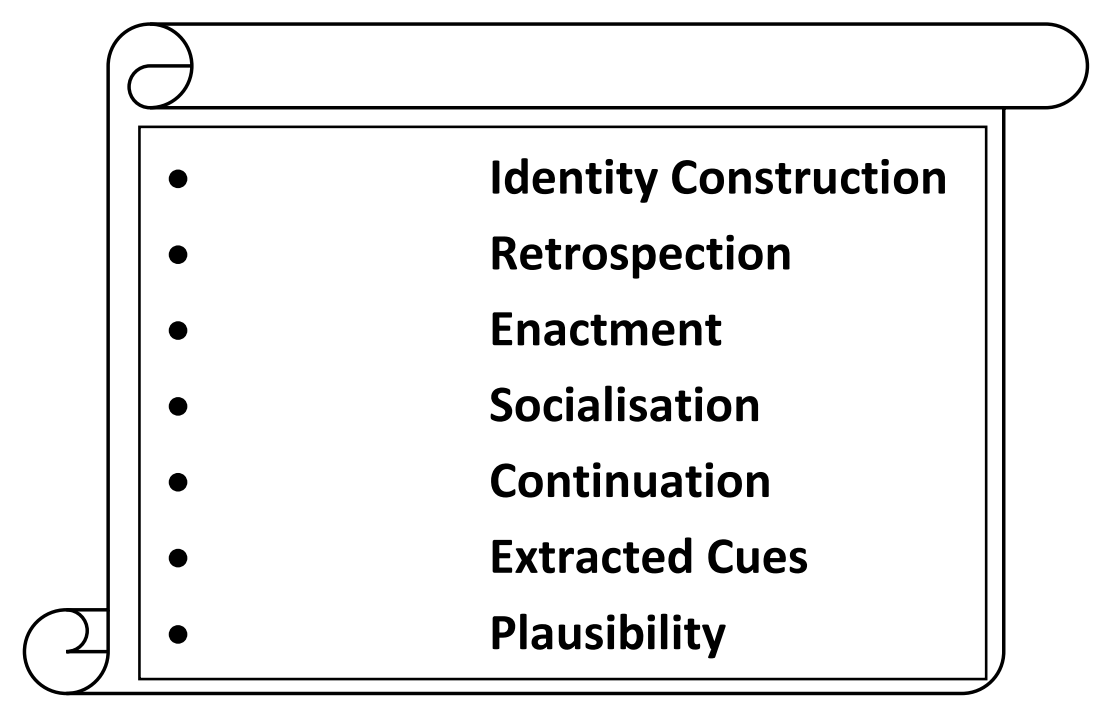

Figure 1 - Diagrammatic Representation of Weick's Sense-making Theory adapted from Weick (1993)

Dervin's approach, on the other hand, is more often depicted as seen in figure 2 below highlighting the significant stages of Situation, Gap and Outcome or Usage. Thus, combining these two sense-making models' assumptions, helped guide this research, especially in constructing the research conceptual framework to help situate and analyse employee OI themes evident in organizations and generated from extant OI literatures, in a bid to understand the concept from an SME context, with training interventions acting as a mediating factor. 


\section{Context :}

- Power strucures

- domain knowledge systems

- cultures and communities
Outcomes :

- help, hindrances

- functions, dysfunctions

- consequences, impacts, effects

- future horizons

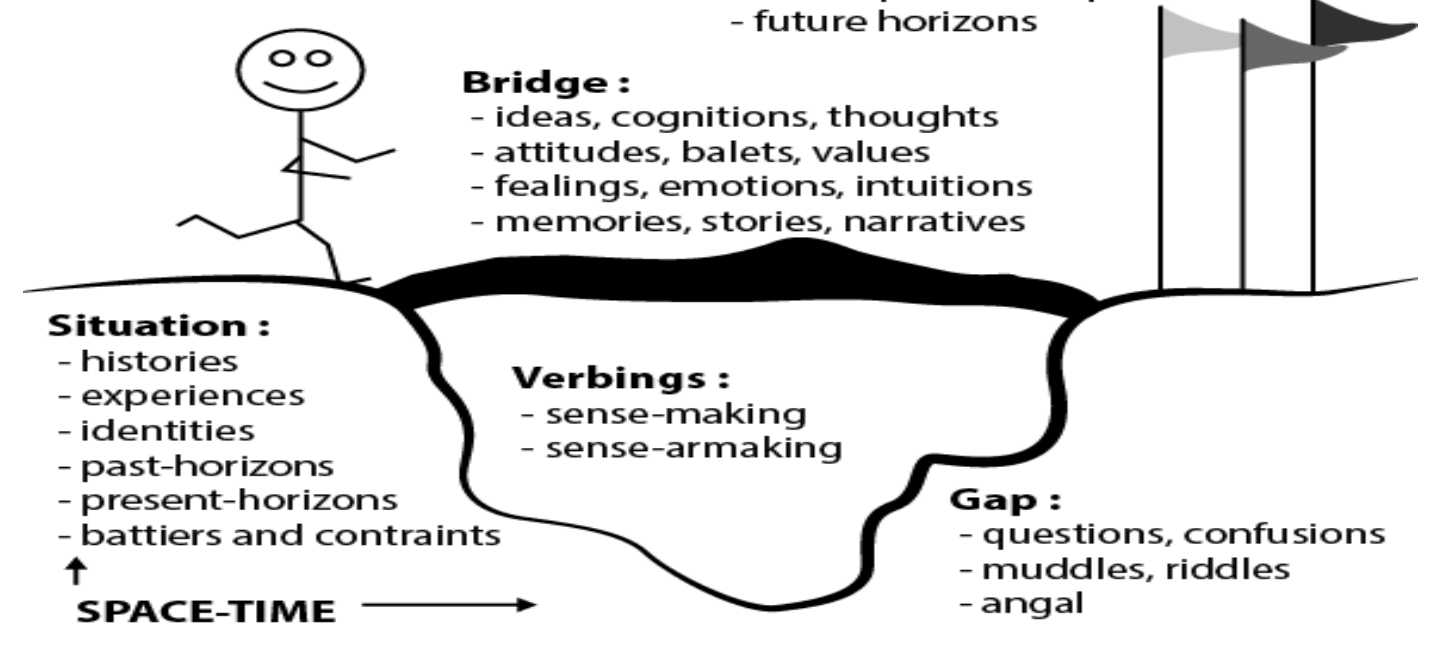

Figure 2 - Diagrammatic Representation of Dervin's Sense-making Theory adapted from Snowden (2008)

The model explores the rationale that humans generally seek information when they encounter an obstacle, or gap, of some kind that they see as a block in their life path, and to bridge that gap the individual seeks or revises information, methods, and new approaches that they find helpful.

Thus, these two above models helped guide the research particularly in analysing the themes generated into meaningful constructs in relation to OI. To understand the framework better, however, a brief comparative discussion on the Weick(1993) and Dervin(1999) models of sense-making is provided below.

Weick's approach to sense-making mirrored the significance of situational awareness and an element of group activity plausibly leading to normative organizational sensemaking. On the other hand, Dervin's approach focused on the alignment of individual perceptions in relation to communication within organizations on ideas within organizations. Analysing these models further, the researcher highlighted elements of each model that were found to have similar meanings, and these were reconstructed into converging concepts that formed the building blocks of the $\mathrm{OI}$ research framework used within this research. 
Dervin's model has six main concepts, namely: Context; Situation; Gaps; Bridges; Verbings; and Outcomes, which are all subject to Variability of Time and Space, while the Weick model has seven main rallying themes, namely: Identity Construction; Retrospection; Enactment Socialisation; Continuation; Extracted cues; and Plausibility, pointing to the logical rationale that sense making does not take place in a vacuum. It is necessarily aided by the presence of and/or implementation of these concepts combined together in various ways.

The first theme in Weick's model is Identity Construction in which sense making is affirmed to be a matter of understanding who we are in relation to the world around us and in the context of this research the set organization. In addition, Socialisation is our relationship journey with the world figuratively and is synonymously related to the "Situation" concept as defined by Dervin (1993) that emphasises the significance of our histories, experiences, identities, organizational dynamics such as power toggle and the varying time horizons as determinants within an individual's sense making process. Retrospection, according to Weick, is sense-making done in hindsight to make sense of actions that have already occurred, to inform and develop the individual's ability to react to similar future occurrences. Also, this idea is somewhat mirrored in Dervin's Situation and Context themes.

Gap Identification within Dervin's model is synonymous to the extracted cues theme of Weick's model. It is assumed that we can identify a Gap through cognitive perception generated from extracted cues from the situational context we find ourselves in. This Gap is then articulated and expressed through Verbing, another theme within Dervin's model. This theme expresses the "what I say" part of Weick's model. In doing so, we categorise and reinforce cues and their meaning and then add them to our repertoire of retrospective experience. Furthermore, Enactment, which Weick (1988) describes as representing the notion that when people act, they bring structures and events into existence and set them in action, is similar to the Bridge theme within Dervin's model. In some cases, they can even enact 'limitations' upon the system to avoid issues or experiences.

Meanwhile, Continuation means that sense making is a continuous flow, because the world, our interactions with the world, and our understandings of the world are 
constantly changing, and Plausibility enables action-in-context as we simply cannot know everything exhaustively so we have to move forward as best as we can, these being the pre-cursors to Dervin's "Outcome" theme.

Based on extant literature assumptions in combination with Weick and Dervin's sense making theories, an initial conceptual framework was developed and is discussed in detail within the conceptual framework sub-section 2.12 of this chapter.

\subsubsection{Measuring Organizational Identification}

Several OI measurement scales have been developed in the extant literature of OI. One of the most common is the Mael and Ashforth (1992) Ol six-item scale that tries to measure the extent to which employees feel 'belongingness to' or 'oneness with' the organization. Some of the questions are asked in forms such as "The successes of (organization name) are my successes" and "When I speak about (organization name), I usually refer to 'we' and not 'they".

A closely related six-item scale was developed by Meyer et al. (1993) and used by other researchers in related research for measuring affective tone of commitment (Carmeli 2005, Carmeli, Gilat, Weisberg 2006, Freund 2006) or in the Ol literature emotional component of identification (Bergami \& Baggozzi 2000). The scale items included "I really feel as if this organization's problems are my own". "This organization has a great deal of personal meaning for me, I do not feel like 'part of the family' at my organization". It is however argued that this scale is more suited for determining affective commitment rather than measuring OI holistically (Jöreskog, 1993).

Another measure was developed by Edwards and Peccei (2007) based on three separate but closely related factors of OI. These three factors include: categorization of self as an organizational member; integration of the organization's goals and values; and development of an emotional attachment, belongingness to and membership of the organization.

Other measures focusing on employee perception of and belongingness to the organization include the organizational identification questionnaire (OIQ) which is 
somewhat similar to the affective commitment scale and has been popularized in the literature (Boros, 2008).

Despite the popularity of these scales, however, some proponents of alternative scales believe that measures such as the Mael and Ashforth (1992) scale, despite being simple to use, do not capture broadly the various antecedents of employee OI in organizations. Mael and Ashforth's (1992) Ol measure is also said to be narrower and more distinct from the $\mathrm{Ol}$ questionnaire, as the latter has more overlap with the affective organizational commitment. In addition, Mael and Ashforth's (1992) OI measure may be more useful than either the Ol questionnaire when examining or predicting employee extra role behaviour and job involvement. However, the $\mathrm{OI}$ questionnaire is a better indicator of employee intention to leave the organization than either the affective commitment scale or Mael and Ashforth's OI measure (Riketta, 2005).

In this research, however, a seven-thematic adaptation of both the Mael and Ashforth (MA) (1992) Scale and the Podnar, Golob \& Jancic (2011) (PGJ) scale is employed. Depicted in figure 3 below and containing questions like "I feel that the company I work for is a part of me" and "I enjoy interacting with my co-workers" as a guide, the PGJ scale takes into consideration the duality of employee OI, i.e. 'group and individual' within the organization while embracing the perceptive component of the MA scale.

Another rationale for choice of the PGJ scale is its suitability of use in SMEs, as the proponents of the scale used SMEs (more specifically within the creative service industries who are heavily reliant on staff to strengthen their corporate brand, and in turn to reinforce internal identification and commitment) as their population sample (Podnar, Golob \& Jancic, 2011). 


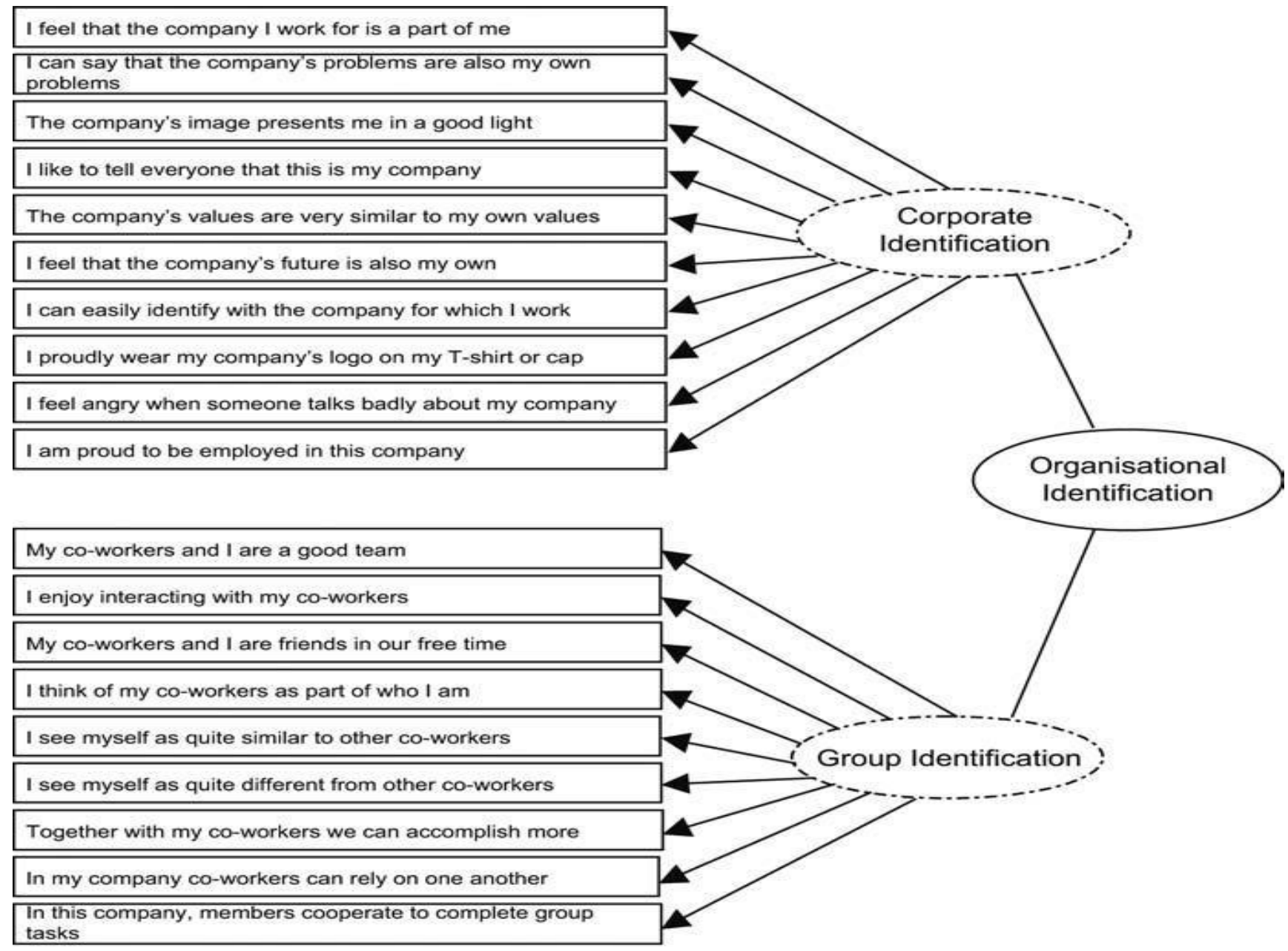

Note: Ovals represent latent variables, and boxes their indicators (items)

Figure 3 - Podnar, Golob \& Jancic, (2011) Ol Measuring Scale

A combination of both adapted scales and Weick and Dervin's sense-making theories formed the basis for the developed conceptual framework in my thesis.

I also employed the analytical Interpretative Phenomenological Approach (IPA) to interpret my collated dataset that captures the lived experiences of the research respondents, since this fits seamlessly with the structural development of the various OI scales that basically explores the perceptions of individuals regarding various factors in their working context(s) as depicted by questions such as 'I see myself as a part of my company'.

This format of questioning themes elicited individualised responses from the research respondents, which was crucial to the validity of the overall study. IPA is concerned with the exploration of the lived experiences of humans through enabling 
individuals to express their meaning of such experiences independently without preconceived categorisations (Smith, Flowers \& Larkin, 2009), and was thus considered most likely to foster genuineness of the research outcomes.

Interpretative Phenomenological Analysis (IPA) was first introduced to the UK in the 90s by Jonathan Smith in response to the continuously evolving field of qualitative research within the psychology study area (Smith, Flowers \& Larkin, 2009). Drawing from the Hermeneutic phenomenology approach (Smith \& Osborn, 2009, p. 97, cited in Hutagalung, 2016), this interpretative phenomenological analysis aims to measure how the participants interpret the personal and social world. Thus, IPA is arguably a suitable approach for exploring how individuals perceive certain situations (Hutagalung, 2016), which in the case of my research is employees' organizational identification. Further rationale for choice of IPA is discussed extensively in the following chapter three of my thesis (see section 3.4, pp. 95-99).

\subsection{The Conceptual Research Framework}

Literature studies indicate that the $\mathrm{Ol}$ process is relatively subjective, as a psychologically activity within an individual's mental framework (Albert, Ashforth \& Dutton, 2000). Thus, Weick (1993) and Dervin(1999) sense-making models are used in my research to understand individual employees' OI processes. Adopting both models gives a more holistic view of the sense-making process.

The external rectangular casing of the framework indicated in figure 4 represents the organizational boundary of the SMEs being contextually discussed, while the cylindrical shape at the bottom end in the casing emphasises the organizational identification process as an individualised employee sense-making process construct within a defined organizational setting.

The two main categories of factors shaping employees Ol process within an organization, namely Employer Situational-Context Markers and Employee Situational-Gap Markers, are depicted within rectangular boxes. The two arrowheads on the upper cyclical end of the cylinder within the framework depict the ongoing interactions between the various identified $\mathrm{Ol}$ markers from extant literature influencing the employee(s) Ol process within a small business organization. 


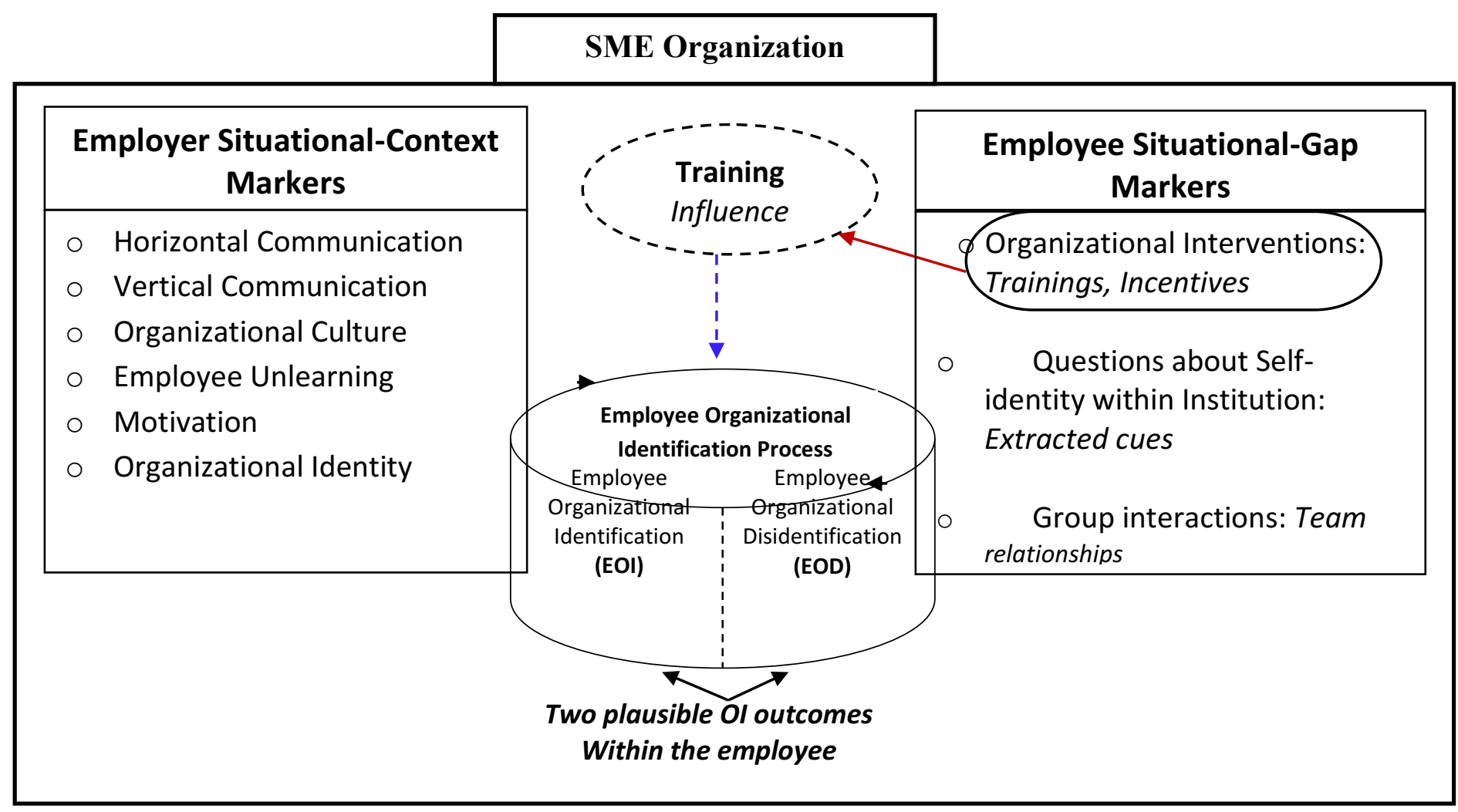

Figure 4 - Organizational Identification Conceptual Research Framework

These two categorisations of $\mathrm{OI}$ markers both contain various factors identified from relevant extant literature as earlier discussed in sections 2.9 and 2.10 (pp. 42-72). These factors acting as $\mathrm{Ol}$ markers are proponents for the development of either employee organizational identification or disidentification status within a small business. Also, an inherent assumption of the framework is that only these two possible outcomes relative to small businesses organizational context can occur, as an outcome of the ongoing interactions between the various $\mathrm{Ol}$ markers highlighted within the framework.

\subsubsection{Employer Situational-Context Markers}

The organizational identification markers within this category are essentially defined as a function of intra-organizational elements that are often beyond the control of the employees within an organization. Evidence from extant literature on Ol studies suggests that both horizontal and vertical communication, power structures defined by the management and leadership within the organization, organizational culture, employee unlearning or their previous experiences and organizational identity are all predictors of organizational identification in organizations (Section 2.9 pp. 42-59). 


\subsubsection{Employee Situational-Gap Markers}

However, OI markers within the employee situational-gap category are essentially factors employees have substantial control over. These are defined as personalised factors which affect organizational identification that are not subjected to elements of external control outside the individual. That is, employees can take proactive actions regarding these factors, as to whether and how they impact on or inspire their $\mathrm{Ol}$ process development. The three employee situational-gap factors identified in the conceptual research framework include: organizational interventions; organizational interactions; and questions about self-identity within the organization (Sections 2.9 \& 2.10, pp. 42-72).

\subsubsection{Employee Organizational Identification and Disidentification}

These terms refer to the two possible employee Ol outcomes proposed by the conceptual research framework which evolved from existing OI literature. Extant studies on OI generally assume development of EOI statuses by businesses in organizations often has positive outcomes or effects on the employees' attitudes and behaviour in the company. EOD is, however, seen as a less favourable organizational identification outcome in businesses, as its development among employees could affect the holistic organizational performance negatively. That is, it is seen as an extreme opposite of EOI.

\subsubsection{Research Questions and the Conceptual Research Framework}

Figure 5 below shows the partitioning or sectioning of the CRF relative to how each section addresses the three main research questions in my study. That is, it tries to establish the interconnectivity between the three main research questions addressed and the three main parts evident in the conceptual research framework (CRF). The functionality of this framework relative to the research is explained in more detail in the subsequent methodology chapter using the research design framework (section 3.6: pp.105-127). 


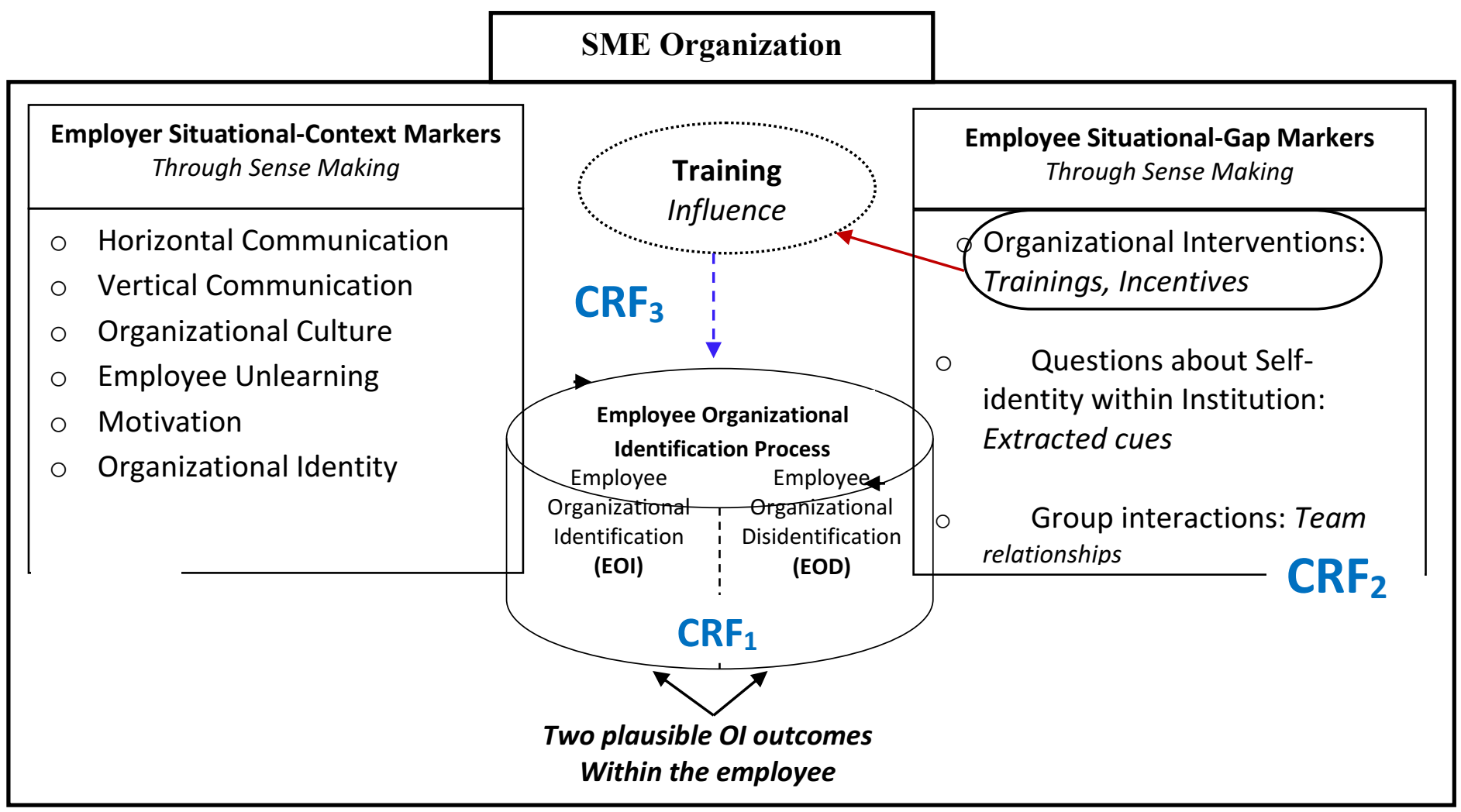

Figure 5 - Sub-sections within the Conceptual Research Framework

\subsection{Chapter Conclusion}

This Literature review chapter has explored various OI related extant literature and explored the nature of the relationships existing between the organizational identification-related factors and organizational identification construct itself. In addition, HRM/D and training studies have been reviewed and the varying significance of training in improving employees' behaviour in organizations as well as specifically in SMEs has been highlighted. The need for further SME studies has also been discussed.

From this review of literatures, a number of factors emerged as strong predictors of employee organizational identification or disidentification statuses in organizations and these factors then formed the basis for development of the organizational identification conceptual research framework. These factors, coupled with the understanding of specified sense-making theories acting as mental models, helped develop the proposed research framework. 
Further brief breakdown of how the conceptual research framework addresses the research questions was provided and this will be explored in more detail in the methodology chapter (Section 3.6. pp. 102-108), with a more definitive research design framework presented, helping to guide the structural design of the research process.

Furthermore, it is essential to note that the Ol markers presented in the conceptual research framework (Figure 4 and 5) are continuously evolving over time and the combinations that are active in the OI process of each employee over time may differ from each other, which explains the essence of the employer situational-context nomenclature used for the two OI markers categories. The framework recognises that every employee in a small business organization is a separate entity and they have their individual $\mathrm{Ol}$ processes shaped by the interactions of different permutations of these $\mathrm{Ol}$ markers over time. This also supports the ideology of sense-making employed within this research (Dervin, 1994).

An implication for this research of the continuous evolution of interaction of the identified $\mathrm{Ol}$ markers is that since the time during the research when the respondents' stories and narratives were analysed, their EOI or EOD statuses could have shifted in response to changes experienced through interaction with the various OI markers identified in the conceptual research framework.

In summary the primary aim of developing the conceptual research framework is to explore these $\mathrm{OI}$ markers and to primarily ascertain whether or not training interventions help equip SME employees in working through their respective OI processes to produce a definitive EOI or EOD status within the firm. 


\section{CHAPTER 3}

\section{Research Philosophy, Methodology and Design}

“The qualitative researcher's perspective is perhaps a paradoxical one...although a researcher's knowledge is always based on his or her positionality... it is to be acutely tuned-in to the experiences and meaning systems of others—to indwell..." (Mullings, 1999 cited in Dwyer \& Buckle, 2009 , p. 55) and “... we have an appreciation for the fluidity and multilayered complexity of human experience... at the same time aware of how one's own biases and preconceptions may be influencing what one is trying to understand...we have an appreciation for the fluidity and multilayered complexity of human experience..."(Maykut \& Morehouse, 1994 cited in Dwyer \& Buckle, 2009, p. 60)

\subsection{Introduction}

This chapter explores the research philosophy and methodology underpinning the design of this study. A sequential narrative of relevant ontological and epistemological positions will be discussed, with emphasis on the Interpretative Phenomenological Approach (IPA) stemming from the Heideggerian phenomenological perspective, which guided the data generation and analysis in this research.

\subsection{Research Philosophy}

The multidisciplinary nature of academic research often leads to complexity and diversity in the philosophical approaches employable in research. Despite this complexity however, it is often crucial for every researcher to justifiably pinpoint their own philosophical stance driving the direction of research undertaken (that is, choose a suitable 'best fit' approach to adopt). As this not only provides clarity to the scheme of the research by eliminating any ambiguousness, but also often serves as a pathway blueprint for similar or related future research studies. This is in alignment with Johnson and Clark's (2006) argument that 'the most important duty of a researcher is to be able to understand the philosophical/approach choice made and justify the use in the context of their research. 
Furthermore, these philosophical approaches have different nomenclatures and categorisations and Fisher (2007) emphasises that having knowledge of them can be harnessed to better inform the research design. Also, understanding in depth the assumptions underpinning relevant philosophical positions helps facilitate the adoption and/or adaptation of an appropriate methodological choice for specific researches (Easterby-Smith, Thorpe \& Jackson, 2012). Consequently, an awareness of the different philosophical approaches is often instrumental in developing and broadening a researcher's innovative mind by adapting other methods outside the researcher's personal experiences and immediate research study area to fit a specific intended research study (Pierret, cited in Thietart \& Wauchope, 2001, p. 3).

In the subsequent sub-section of this thesis chapter, I explore the philosophical significance of subjectivism, which served as the key guide for my methodological choices employed within this doctoral research, in comparison to the objectivism philosophical approach. The contextual benefits and inherent disadvantages of both philosophical positions will also be analysed. Further discussion is articulated on relevant ontological and epistemological positions, theoretical perspectives/paradigms employed in my study with examples provided in the context of the wider business management research studies, to explain these parameters' contextual usefulness and relevance to my study and especially in influencing my research design framework.

\subsubsection{Subjectivism versus Objectivism}

Crotty $(1998,2003)$ categorised Objectivist and Subjectivist philosophies as theoretical perspectives. Researchers with an objective ideology believe in the assumption that 'social entities exist in reality, which is independent of people's perceptions, and also recognise that the real objective world can be understood only through hypothesis testing and experiments' (Robson, 2002, p. 110). Objectivists believe in 'causality'; that is, it relates to factors that lead to observed effects (Remenyi, Williams, Money \& Swartz. 1998, p. 32). The objectivist research process involves formulation of a hypothesis, which is either verified or refuted by the observed effects based on the researcher's understanding of the specific 
phenomenon under study (Leavy, 2014). They then generalise results from ample sample sizes, as is the case in most physical sciences research (Tsui \& Ngo, 2015). Objectivists usually "identify causal explanations and examination of fundamental laws that explain regularities in human social behaviour" (Easterby-Smith et al., 1991, p. 23; Leavy, 2016).

Some of the critics of the objective philosophy, however, pose the questions: 'how many behavioural attributes (which are often inherent in the individual) can be determined by a researcher who is a separate entity in his/her own right and external to the individual under study?' and: 'how really predictable is human behaviour in general?'; that is, human behaviour is quite unstable, thus unpredictable (EasterbySmith et al., 2001; Leavy, 2014)

Subjectivism, as a different philosophical viewpoint, attempts to address these questions by assuming that 'people construct their own meaning about social entities in different ways, even in relation to the same phenomenon' (Remenyi et al., 2005). Subjectivists argue that researchers cannot distance themselves from what is being observed; the study's subject matter; and the methods of study. This is because the researcher usually has an inherent bias, as a result of their other personal attributes, such as their background, status, interests, beliefs, skills, values and resources (Hunt, 1993).

Thus, subjectivist based research outcomes are guided by interpreting observations the researcher witnessed, by focusing on the meaning of social phenomena rather than their measurement. A subjectivist aims to understand, then explain a problem with respect to a specific context. Subjectivists also believe that research problems are often related to meanings individuals attach to a phenomenon rather than a question of causality argued by objective researchers (Easterby-Smith et al., 1991; Hughes \& Sharrock 1997).

Both objectivism and subjectivism are often adopted in business management research. Objectivism researches often study structural processes and procedures within business organizations (Walliman, 2006, p. 16). These could be operational procedures or techniques within the firm in which there is a specified hierarchical 
structure involving processes that are independent of all other external factors related to it. Subjectivism, on the other hand, acknowledges the significant possibility of external factors influencing organizational operational processes or procedure (Crotty, 1998; Walliman, 2006).

A typical example could involve research into improving the productivity of a paint manufacturing company. A researcher with an objective view might not put into consideration the influences of some unpredictable aspects of employee behaviour, such as that interaction with their manager may have either a negative or positive effect on the overall productivity of the company, and focus on improving the physical procedures or production techniques. While a subjectivist will likely explore the possibility that personal biases could influence the behaviour or action of the individual employees and their perception about productivity topic in question. As no matter the structural improvements undertaken within the company, employee productivity might not necessarily improve, if the employees themselves are not convinced to either improve or see the usefulness in the newer production techniques being introduced.

One significant limitation of objectivism-based research is demonstrating the viability of the generalised assumptions made of reality, especially in social research where human actions can often significantly influence study outcomes directly or indirectly. So, these generalised assumptions may not necessarily hold true in all research cases. Avoiding this limitation posed by objectivism-based studies is a key rationale also for my choice of subjectivist study and essential to my research study. This is significantly important as my research focused on exploring human behaviours and feelings rather than structural processes. This exploration is coupled with the oftenunpredictable nature of human behaviour and because generalised assumptions may not necessarily hold true for all research respondents considered while exploring employee Ol. Hence, it was rationally imperative to explore each respondent's narrative firstly on its own individual merit subjectively, before scaffolding more definitive assumptions generated from the respondents' data set. This rationale also influenced the procedural structure for data analysis, detailed later in section 3.7(pp.122-124) of my thesis methodology chapter three. 
On the other hand, subjectivism has also been criticised based on its frequent inability to generalise and apply outcomes of research to other samples, as each research carried out is often a reflection of what is happening in the research context at that point in time only. Despite this notion, I still chose to employ a subjectivist view within my research. My research was able to generate in-depth data on the subject matter of $\mathrm{Ol}$, contributing to the general understanding of $\mathrm{Ol}$ among researchers, whereas most of the limited academic literature currently available on Ol has used an objectivist method of inquiry to define the study outcomes.

Also, it can be argued that objectivists might be more concerned about outcomes, albeit governed by human actions, while subjectivists are more likely to be focused on understanding the nature of the human understanding or interactions involved in the subject under research. Employing this subjectivist view enabled me to make interpretative sense of each research respondent's perception about Ol. Hence, my research draws on the strengths of the exploratory study, OI, as a psychological construct, the respondents' knowledge about what $\mathrm{OI}$ is, and their perception on how training and other organisational and individual factors like ethical leadership and perceived in-company worth, affects their individual OI process.

Thus, given their different advantages and shortcomings, neither of these two philosophical positions can be argued to be most appropriate for all research scenarios, and both objectivists and subjectivists are active researchers within the business management research area. Each researcher only defines, rationalises and justifies their own philosophical choice per research done (which I have done in the context of my research) and their rationale could be based on such as the paucity of literature on the research subject and research questions to be answered (Holden \& Lynch, 2005).

\subsection{Ontological Assumptions}

Central to the philosophical debate of the research process among researchers are the understandings of 'Ontology' and 'Epistemology' and what it is actually possible to discover by research (Fisher, 2007, p. 14). Ontology is concerned with the 'what' question, that is, what the actual nature of reality and existence is, while Corbetta 
(2013, p. 12) and Wisker (2008, p. 67) also define ontology as 'being in the world.' Ontology explores how reality is perceived by a researcher given that our understanding is framed by what our perceptions are about the subject matter. These perceptions however often differ from person to person.

According to Easterby-Smith et al. (2012, p. 9), ontology is generally categorised into four main types, based on the assumptions as to how the nature of reality (truths and facts) is perceived. These categories include realism; internal realism; relativism; and nominalism. The first two variants: realism and internal realism, are often employed by natural scientists and fall under the objectivism category and thus possess relatively similar binding assumptions. The assumption is that social entities exist as facts and these facts/truths existence are independent of other social entities associated with their existence (Saunders et al., 2012). Relativism and nominalism variants, on the other hand, are entrenched within the subjectivism approach to research which assumes that 'facts and truths' about reality are perceived meanings derived from social entities specific to the individual studying the specific social entity and thus truths and facts are perceptions of human reality and are 'man-made' (Crotty, 1998; Easterby-Smith et al., 2012).

As my research involved obtaining different employees' perceptions about Ol via interviews, interpretivism, a variant of the relativism ontological position, was adopted in my research to understand the influence of training activities on individual employees within SMEs as well as establish the appropriateness of the OI markers situated within the research conceptual framework. Since relativism proposes that facts depend on the viewpoint of the observer, rather than approaching it in a procedural or scientific form (Easterby-Smith et al., 2012), my rationale in using the interpretivist approach was based on belief that the eventual outcome of my study would be influenced by the varying perceptions of the research respondents as interpreted by the researcher, within the set organizational context. Furthermore, variants of the relativism paradigm, such as interpretivism, often also use small samples and generate in-depth insight of individuals' perceptions of reality, which is appropriately demonstrated in the structuring and data generation techniques used in my research. 


\subsection{Epistemological Stance}

The philosophical debate on epistemology explores the question of what and how 'acceptable knowledge' is acquired within specific fields of study. It answers the 'how' question which inquires about the acceptable ways and best practices employed in inquiring into the nature of the world (Saunders et al., 2012; Easterby-Smith et al., 2012). This also resonates with the Baker and Foy (2008) assertion that epistemology is understanding what does or what does not constitute warranted knowledge, which significantly informs the choice of methods or techniques employed in gathering data and information (facts and truths) in a research study. Consequently, the varying understanding derived from various epistemology assumptions has led to the nomenclature of diverse epistemological paradigms in social research.

Taking an understanding of this, my epistemological position was based on knowledge acquisition through the interpretative understanding of respondents' livedexperiences and perceptions of the research respondents on the subject matter of OI. Hence, the experiences and perceptions of the respondents are interpreted through a process of de-construction and re-construction to make meaningful deductions (Laverty, 2003), which is essentially the interpretivist stance I employed in this research. My choice is justified based on the recognition and importance of inferential and constructive thinking, that is, making meaning and sense out of the research study and the data collected as the research progresses.

Also central to the philosophical assumptions of interpretivism is subjectivism or the subjective inference, which is synonymous to the gnostic extreme of Fisher's (2007) epistemology framework adaptation. This 'Gnostic' notion assumes that the world is largely unknowable and that what we know is of our own construct or perception and is patchy. This is also so often referred to as Social Constructionism (Easterby-Smith et al., 2012) or constructivism (Pierett cited in Thietart \& Wauchope 2001).

As earlier iterated, this branch of research paradigm evolved as a result of the uncertainties and unpredictable nature of social entities, particularly those of human 
behaviour as opposed to other natural scientific phenomena whose behaviour can be scientifically predicted. According to Pierett (cited in Thietart \& Wauchope 2001, p. 14), however, some see interpretivism and constructivism as being different despite having similar ontological assumptions of relativism as discussed in section 3.2. To them, in social constructivism reality is essentially constructed, while interpretivism seeks to understand and interpret reality and is hence the apt choice for adoption within this thesis.

Interpretivism has however also been criticised on the premise that the inability to make generalisations from knowledge based on belief in the individuality, uniqueness and behavioural characteristics of human actors defeats the entire purpose of research itself (Corbetta 2013, p. 26). Also, the concentrated focus on individuality often shuns the importance of other social institutions, actors and interactions in play within the same research construct. Though not subjected to in-depth scrutiny based on the interpretivism research paradigm, these shunned actors could still have significant influence on the outcome of the specific research (Corbetta, 2013, p. 26).

Despite this disadvantage, the researcher chose to use this paradigm as it fits into the exploratory concept of her study which involved interpreting the perceptions of research respondents based on their experience, and thematically contextualising these perceptions in relation to the concept of $\mathrm{O}$ to form meaningful assumptions.

In line with my interpretivist views, I employed the interpretative phenomenological inquiry approach to explore the perception of the respondents on OI. As a research philosophy, phenomenology is said to be the study of experience (Husserl, cited in Langdridge, 2007 , p. 10). Initially it was understood to be the study of how things appear to people, that is, how people experience the world either singularly or collectively (Schutz 1967 cited in Boland, 1984; Fisher, 2007, p. 14); in other words, distinguishing between the objective and subjective ways of understanding knowledge and social constructs. However, in recent times it has been increasing defined to relate similarly with the concept of interpretivism (Crotty, 2003, p. 83). It is now seen as the study of people's subjective and daily experiences. 
Further research into understanding the nature of phenomenology as a research approach highlighted the Husserlian phenomenological approach to research, which necessitates the researcher to bracket own world view, knowledge and experience when describing the experience of the research participant (Langridge, 2007). While another school of thought, presented by Heidegger (Husserl's student), views phenomenology differently and advocates for an interpretative approach where the researcher uses own knowledge and experiences to interpret the experiences of the research participants. Heidegger further argues that 'whenever something is interpreted as something, the interpretation will be founded essentially upon forehaving, fore-sight, and fore-conception. An interpretation is never a presuppositionless apprehending of something presented to us' (Heidegger, 1978, pp.191-192).

Thus, as the researcher I based my phenomenological stances on the Heidegger version which translated as ensuring that my personal pre-conceptions, assumptions and even my prior experiences were employed as tools to form a basis for interpreting the data I collected; as affirmed by Laverty (2003), 'we are constructing the world from our own background and experiences'. Furthermore, while it is essential to always ensure priority is given to the respondents' perception and experiences, it is also very important to recognise the influence of the researcher's own experiences and pre-conceptions on qualitative phenomenological research inquiry, albeit with a clear acknowledgement that the interpreted data must be valid and trustworthy. Even though Gadamer (1977) viewed bracketing as 'impossible', a certain degree of bracketing was included in this research pursuit to guide against my own pre-conceptions or experiences overshadowing my understanding and interpretation of the interview respondents' perceptions.

The phenomenological approach employed as advocated by Heidegger was wellmatched to my research design and conceptual framework as it allowed room for sufficient subjectivity while interpreting respondents' data collated on the subject matter of $\mathrm{OI}$ being researched. In addition to the subjectivity aspect, Heidegger also introduced hermeneutics into the phenomenological research approach (Gadamer, 1977). 
Hermeneutics is a process of interpreting human actions, utterances, products and institutions that is often adopted in analysis of textual contents. It is the theory of interpretation' with its origins attributed to the era of interpretation of biblical texts in seventeenth century Germany (Easterby-Smith et al., 2012). One contended view in relation to hermeneutics is that it is often impossible to understand in totality the external conditions under which the writer of such texts was working at the point of writing, which would have had a great influence on the implied meaning of the text at that point in time (Ricoeur, 1981). Thus, it would be ideal for the reader and the author to have a discourse about the writings at each point in time, which would in reality often be impossible, especially in the case of historical research. Hence, there may be no out-rightly correct interpretation of the writing, as the reading and writing are always context dependent and, as a result of temporal distance, context setting may not be obtainable or verifiable.

These aforementioned disadvantages notwithstanding, the Heidegger hermeneutics phenomenological research approach still influenced the methodological design of this research in conjunction with interpretivism methodology, as they form the bedrock on which the Interpretative Phenomenological Analysis (IPA) was developed. Therefore, an interpretation of the textual articulations of the perceptions and lived experiences of the research respondents is an understanding of the understanding of the experiences, as told by the respondents. This is so because, in narrating their stories or experiences, the respondents were simultaneously getting an understanding of these experiences relative to the context of Ol. Hermeneutic phenomenology also allowed the interpreter who is also the researcher to 'put herself in the place of the other' and her own experiences inform the interpretation. Thus, within my study, I as the researcher was not a neutral observer or a participant and instead, my own experiences and pre-conceived assumptions were completely embedded in and valuable to the interpretative process, a practice often employed by other qualitative researchers (Langdridge, 2007; Smith et al.,2009; Willig, 2009).

\subsection{The Interpretative Phenomenological Analysis Approach}

The thesis methodology adopted is Interpretative Phenomenological Analysis (IPA). Developed from Husserl's Hermeneutic phenomenological strand, IPA, according to 
Smith, Flowers and Larkin (2009), is concerned with exploring the lived experiences of humans with the understanding that the meaning of such experiences can be expressed by the individuals in their own way without any pre-defined categories. IPA is also a strongly idiographic approach concerned with detailed analysis of the case either as an end in itself or before moving to similarly detailed analyses of other cases. The method is employed within the data generation, analysis and discussion phases of this research, as it embraces the significance of subjectivity. Herein, the researcher engages with the research by striving to derive understanding and make meaningful deductions from the lived experiences of the research respondents (Singh, 2016).

According to Smith (2004), one interesting characteristic of the IPA method is that of dynamism as it focuses on a first-hand exploration and interpretation of the experiences of the research respondents. In this regard, the respondent is viewed as the 'expert', shifting power from the researcher to the respondent as it is the latter who decides what they tell the researcher. IPA also explores how participants perceive and make sense of their experience, and understand the meanings of that experience (Smith, 2008). IPA assumes exploration of experience in its own terms and that there is a relationship between individuals' verbal accounts and their cognitions and emotions, which explains why it is phenomenological in nature (Smith et al., 2009; Smith \& Osborn, 2003).

Another advantage of IPA is that it engages the researcher as an active participant with the research respondents in interpreting the information volunteered by actively probing and seeking clarification of understanding, thus making the process highly interactive (Smith, 2004). Also, IPA can be used to facilitate reading between the lines to understand the experiences of the participants as well as the impact of these experiences on the participants. Other advantages include acquiring mastery of skills needed to implement the process appropriately, such as being able to demonstrate self-reflexivity, constructing interpretations of respondents' narratives of their experience, questioning how those interpretations were arrived at, and reading between the lines. To this end, I read a number of IPA method related journals, articles as well as other doctoral theses that have attempted to use the method to design their study. 
Other obstacles that could arise include how to manage the researcher's own preconceived ideas about OI, with the respondents' own narratives, as knowing how to balance the two goes a long way in verifying the validity of the research. Using IPA as a methodology however helped to overcome this challenge, as the very use of IPA helps to ensure that interpretations made by the researcher are fairly influenced by own prior understanding of the subject-matter in the research context, which in this case is organizational identification (Smith et al., 2009). Nevertheless, I carefully structured the analysis process by following a stepwise sequence of research design, as well taking time to seek advice from other researchers in my field that have at some point used IPA methodology in their research work, an effort that also proved very useful in getting referrals to extant literatures that helped further my understanding of the methodology.

Furthermore, the process was very time consuming, especially in the transcription and interpretative stages. I initially planned to solicit the help of a proficient data transcription service to speed up the data analysis stages. However, due to certain constraints, particularly financial, I eventually transcribed the interview recordings myself, which proved beneficial as I was able to better familiarise myself with the textual data set. Also, during the previously conducted pilot research study, I was able to develop my confidence and expertise in interviewing respondents. It should finally be noted that according to Smith (2004) the findings derived may not be generalisable to a much larger population.

Another justification of the use of IPA in this thesis is that it not only fuses almost seamlessly with the ideology of the sense-making theories underpinning the conceptual research framework in terms of Ol being a psychological concept, but it also recognises the essence of interpretation from the researcher's perspective. That is, IPA helps the exploration of an individual's perception on a phenomenon and rather than produce an objective recount of such experiences, the researcher subjectively delves deeper to understand these research respondents' experiences/stories (Smith et al., 1999) and then interprets them to make meaningful map threads, showing how each experience is related to the phenomenon under scrutiny. 
One of the novel features that this research adds to academic literature is the use of IPA to understand the Ol concept within the small businesses management field of research, as to the best of my knowledge no previous study has done this. Whereas in other fields of research such as marketing, leadership, health management and engineering there is an abundance of OI and IPA studies. Moreover, other related studies were conducted within an academic institutional context as opposed to the SME context that is the focus of this thesis.

Furthermore, though widely used in psychological studies, especially within the medical psychology and engineering research fields (Smith et al., 2009), IPA is also increasingly used as a contemporary methodology within business management. The method helps explore the lived experience of the research respondents and its influence on their Ol process. IPA also provides in-depth data from the research subjects, obtained in their natural environment (Bryman \& Bell, 2003). This means that the researcher interacts with primary actors, to gain better understanding of subject matter (Denzin \& Lincoln, 2003). Thus, the narratives of the research respondents are their own stories they have lived or are living through.

The review of relevant literature review on OI, complemented by my own working experience in SMEs, has led me develop a pre-conception that training programmes in organizations could often have a significant impact on the development of $\mathrm{Ol}$ among employees and that certain factors, known as markers within this thesis, can significantly contribute to the processes through which employees construct either deviant or normative OI.

The understanding of the IPA research framework highlights that the possibility of an in-depth interpretation depends on shared common grounds between the interpreter and the interpreted (Smith et al., 2009). Within the context of this study, the common grounds include the workplace structure (both the respondents and researcher work within SMEs) country of residence (the respondents and researcher currently live in the UK) and participation (both the respondents and researcher have at some point participated in various training programmes within their companies). 
In addition to interpretivism, phenomenology and hermeneutics, IPA is also underpinned by idiography which opines on the 'particular' (Smith et al., 2009). IPA works with small samples that have been purposely selected with recognised homogenous traits. Therefore, IPA aims to understand "how 'particular' experiential phenomena have been understood from the perspective of particular people, in a particular context" (Smith et al., 2009, p. 29). In relation to the current research the 'particular' may be identified as:

particular phenomena - Training influence on organizational identification(OI), particular people - employees,

particular context - SMEs in the UK.

From the review of relevant literatures related to $\mathrm{Ol}$ and at the time of writing up my thesis, to the best of my knowledge, while there have been some studies on $\mathrm{Ol}$, no bespoke studies were found in which training is explored as a mediator for the formation of $\mathrm{OI}$ statuses in small business organizations. Neither have any previous studies combined Weick and Dervin's sense-making theoretical perspectives to develop a conceptual framework in the exploration of $\mathrm{Ol}$ as an organizational behavioural concept. The current researcher considers that combining these methodological and theoretical models will help this study explore and understand the concept of $\mathrm{Ol}$ and showcase how significantly it can affect organizational business management practices.

Furthermore, though idiographic research often embraces the use of small samples, Tsoukas (1989) argued that such studies are able to make general claims about the world. My study will likewise argue that its claims are pertinent to the general situation of most SME employees located within the UK. The findings may then be subsequently used to provide bespoke information to businesses that are interested in improving their employees' OI levels to conform to the long-term strategies of the business. This will enable such SMEs to tailor their training activities most adequately to achieve the intended results.

In addition, the practicalities of investigating the research topic are informed by personal competence, experience, time, funding, availability and willingness of research respondents, especially given that the interview sessions are conducted with different employees in different organizations but all with the common denominator of working for SMEs within the nation's retail or education sector. My 
previous work experience in SMEs, as previously mentioned in chapter one of my thesis, also aptly fits with the IPA methodology I employed for the study and helps inform the interpretative and analysis stages of my doctoral thesis.

\subsection{The Pilot Study}

Some significant periods at the initial stages of my research were dedicated to exploring relevant organizational behaviour, organizational identification, small businesses and training related literatures, in addition to conducting an exploratory research sample pilot study, which helped in ascertaining the viability of researching the OI construct in relation to training within an SME context.

Following on from my decision to employ the IPA method and aided by my understanding of the Dervin and Weick sense-making models, based on exploring the perceptions and lived experiences of research respondents and an understanding that an objective reality can be varied by perceivers' sense-making processes (Smith et al., 2009; Weick, 1993), which suggested that integration can be achieved through constant and fluid negotiation between self and organization (Scott et al., 1998), a pilot study was conducted. The use of the sense-making models with the IPA methodological approach helped to thematically ascertain that the construction of individual employees' OI via their lived experiences is continuous and can be influenced by specific organizational factors or activities.

The pilot study explored the significance of training (often used as a regulatory tool) as a mediating factor in the development of Ol status by five (5) small business organization employees. The pilot study outcomes identified some interesting factors relating to the research respondents' perception of engaging in training activities implemented in their workplaces. The results from the pilot study also highlighted the possibility that varying levels of $\mathrm{Ol}$ statuses can exist, which resonates with the categorisations of $\mathrm{Ol}$ statuses proposed by various other scholars (Dukerich et al., 1998; Elsbach \& Bhattacharya, 2001; Kreiner \& Ashforth, 2004). This resulting rationale ultimately led to the modelling of a more in-depth exploration in the main study and hence the resulting preposition of $\mathrm{Ol}$ as a vector quantity with both magnitude and directional components, as presented in the concluding chapter five. 


\subsubsection{Modelling the Pilot Study}

Based on availability of willing research participants and proximity to these respondents, five initial interviews in total, each lasting between 35 and 50 minutes, were conducted for the pilot study. From this set of piloted study interviews, five key categorisations, developed from ten emergent themes, were modelled for comprehensive analysis of the outcomes of the pilot study.

The ten emergent themes included: Training definition; Motivation for Training; Motivation during training; Receptivity to Training; Notion of Forced Training; Personal Training Needs; Consensual participation; Post-training use; Individualised Training Costs and Training Environment. Further analysis of these themes resulted in five key categorisations of pilot study outcomes:

- How Training is defined;

- Typologies of Organizational Trainings;

- Training receptivity pre-, during- and post-training activities;

- Motivation for Training;

- Post-training use and cost.

\subsubsection{Pilot Study influence on Main Research Design}

Conducting a pilot study specifically helped to inform the conduct and processes I employed for my main research study design. In particular, the initial pilot helped to inform my decision to holistically explore other factors that could potentially influence employee OI process in SMEs other than training interventions, as themes emerging from the pilot very much suggested the significant importance of other inherent actors within the employee $\mathrm{Ol}$ process and hence $\mathrm{Ol}$ status formation. Thus, the main study incorporated and explored the impact of sizably greater number of $\mathrm{Ol}$ markers other than training interventions that was considered in the initial pilot and indeed birthing the nomenclature of 'OI Markers' introduced in this thesis.

The pilot study also proofed useful in deciding to use the semi-structured form of interviews employed in the main study. This proofed particularly helpful, as I was able to more succinctly thematically evaluate the data collected from the main research respondents and the process of finding common themes in the data 
analysis stage was more manageable. Table 1 below detail the demographic characteristics of the initial pilot respondents.

Table 1 - Demographic Distribution of the Pilot Study Respondents.

\begin{tabular}{|c|c|c|c|c|}
\hline $\begin{array}{c}\text { Respondent } \\
\text { Numbers }\end{array}$ & $\begin{array}{c}\text { Interview Timings } \\
\text { (Mins) }\end{array}$ & Gender & Actual Completion Date & Age \\
\hline \hline 1 & 70 & Male & $02 / 2015$ & 45 \\
\hline 2 & 45 & Male & $02 / 2015$ & 28 \\
\hline 3 & 68 & Male & $06 / 2015$ & 32 \\
\hline 4 & 58 & Female & $06 / 2015$ & 32 \\
\hline 5 & 54 & Female & $07 / 2015$ & 25 \\
\hline
\end{tabular}

\subsection{Main Research Design}

Having explored the philosophical background and methodological stance of the research as well as a description of the initial pilot study, this section focuses on explaining in-depth the methodical procedure adopted to collate and analyse the textual data set for the main research study.

Influenced by the relevant reviewed literature and outcomes of the pilot study, the main research structural design was developed to encompass the relevant components of the main research objectives and questions under study. The decision was also taken to expand the sectoral scope of the research, which was originally intended to be sited in SMEs in an educational setting which was as at the time of the pilot study the sector in which the researcher worked. Thus, additional SME sectors were examined during the actual textual data collation.

Research design, according to Creswell (2009), is essentially framed within the confines of three elements: philosophical underpinning, methodological stance and chosen methods of research. The research topic along with the researcher's personal experiences and training also inform the research design (Creswell, 2009). Thus, it suffices to say that each research design procedure applied in particular research should be equally rationalised within compatible philosophical and methodological ideologies. 
Situated within the subjectivism philosophical spectrum, IPA is employed within this research as a qualitative method of research. IPA research method connotes a dynamic process of interpretation wherein the researcher attempts to gain a reasonable perspective of the research participants' experiences (Smith \& Osborn, 2003). Also, due to its inherent features or characteristics, IPA is a particularly useful method to dissect complex inherent human processes such as the subject of $\mathrm{Ol}$ under investigation (Smith et al., 2009). Considering the reasons explained above, IPA was considered a suitable approach to explain the individualised perceptions of the research respondents in relation to how their Ol process is affected within their various organizations.

Following on from the chosen methodology, the research framework structure illustrated in figure 7 (section 3.6.1, p. 106 ) shows the interconnectivity between the main research objectives, the three research questions, the seven sub-research questions (SRQs) developed from the main research questions using guidance from the Mael and Ashforth and PCJ OI scale. This is followed by rigorous aggregation and then interpretation of these SRQs, which finally funnels into the three main sections of the conceptual framework indicative of the initial three research questions.

Choice of sample size was mostly purposive in nature and the eventual fifteen respondents used were indicative of the higher percentile range of respondents often used in IPA studies. This sample size rationale resonates with Smith and Osborn's (2007) notion that "there is no right answer to the question of the sample size. It partly depends on several factors: the degree of commitment to the case study level of analysis and reporting, the richness of the individual cases, and the constraints one is operating under".

Smith and Osborn (2007), went on to substantiate this by pointing to the fact that IPA studies are often done using small sample sizes, as individual analysis of interview transcripts take a rather elongated time and, most importantly, the research aim is to explore in depth the perceptions and meaning assigned regarding a phenomenon by a specified group rather than making premature generic claims. While contradicting the quantitative research rationale that research is of little use if generalisable assumptions cannot be made, this particular study chose to affirm the rationale of 
qualitative researchers that whereas the breadth of quantitative analysis is often superficially defined with almost no cognitive elements evident, in-depth study is important in understanding research phenomena and creating realistic and feasible solutions to issues arising.

Following on from the above rationale, as identified by Smith and Flowers (2007), IPA researchers often use a fairly homogenous sample and through purposive sampling, IPA researchers "...can find a more closely defined group for whom the research question will be significant. How the specificity of a sample is defined will depend on the study; in some cases, the topic under investigation may itself be rare and define the boundaries of the relevant sample. In other cases, where a less specific issue is under investigation, the sample may be drawn from a population with similar demographic/socio-economic status profiles" (Smith \& Osborn, 2007).

Thus, fifteen respondents from diverse industries of the UK economy were interviewed. The common denominators were that all worked within a small business organizational context, the site of research, and had undergone one form of training or the other within their current business organizations. Anonymised names were used during the analysis stage of the research in compliance with research ethical standards and a tabular illustration showing the demographic details of the interview participants is included in chapter four of the thesis.

Two main stages of analysis were carried out after initial transcription of the audiorecorded interviews. While the first stage provided unrefined detailing of various findings of the research related to the sub-research questions, the second stage of analysis produced the more refined, eventual thematic outcomes of the research in relation to the research subject of $\mathrm{Ol}$ and training. 


\subsubsection{Research Design Framework}

This section uses the aid of a schematic diagram to explain the thesis research design and how the data collection and analysis process conforms to answering each of the three research objectives using the conceptual framework proposed previously in chapter two.

A systematic deconstruction that is breaking down into small componential elements of the three main research questions (RQs) generated from the primary research objective $(R O)$ is employed to arrive at seven sub-research questions (SRQ). Figure 7 depicts the Research Design Framework (RDF).

These SRQs form the basis on which the semi-structured interview questions were formulated. Findings from the collective composition of SRQs is then critically analysed in-depth to directly validate components of the conceptual research framework labelled $\mathrm{CRF}_{\mathrm{x}}$, where the subscript number $\mathrm{X}_{1}$ represents sub-sections of the conceptual research framework (CRF) addressed as shown in figure 5 (see page 84).

As shown in the generated RDF representational equations below, different combinations of SRQs represents how each $R Q$ is addressed by the RDF:

$$
\begin{aligned}
& R Q 1=S R Q 1+2 \\
& R Q 2=S R Q 3+4 \\
& R Q 3=S R Q 5+6+7
\end{aligned}
$$

For example, to address $\mathrm{RQ1}$, responses of research respondents to interview question categories SRQ1 and SRQ2 were jointly critically analysed to arrive at a definitive research result specific to $R Q 1$. This procedure is repeated for other two RQs categories. Thus, for RQ2, respondents' responses to interview question categories SRQ3 and SRQ4 were critically analysed to address RQ2, while SRQ5, SRQ6 and SRQ7 were collectively critically analysed to arrive at a definitive result for RQ3. 


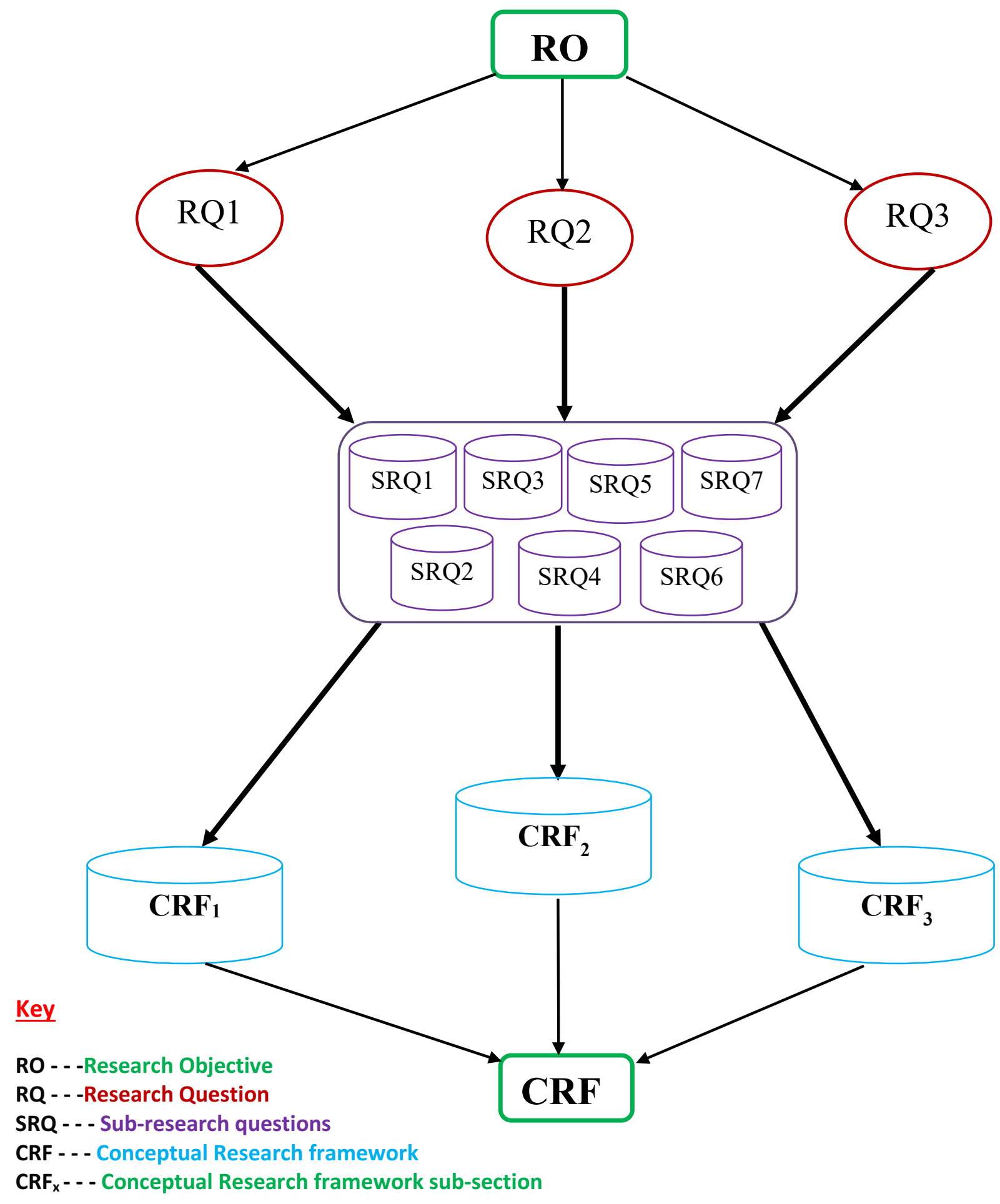

Figure 6 Research Design Framework 


\subsubsection{Research Objective and Questions}

Van Manen (2007) posits that a truly phenomenological research must ask what something is 'really' like in terms of the nature of the lived experience of the research subject(s). Thus, this research addresses the topical issue of Organizational identification: Exploring the use of training as an employee identification marker in SMES.

To further simplify this topic however, the research design process involved developing three main research questions from the key research objective of exploring the use of training interventions as an employee organizational identification maker within SME. This three research questions were then simplified to derive the seven SRQs.

\subsubsection{Main Research Questions}

1. What does employee organizational identification mean within SMEs?

2. What are the underlying markers of employee organizational identification in SMEs?

3. How do training interventions influence the employee identification process in SMEs?

\subsubsection{Sub- Research Questions}

1) What is the perceived understanding of OI within employees of SMEs?

2) How is Ol characterised by employees of SMEs?

3) What are the perceived markers of employees' OI in SMEs?

4) Is training perceived as an employee OI marker within SMEs?

5) If training is an Ol marker, what degree of influence does it wield in the employee OI process?

6) How is training's degree of influence as an Ol marker characterised by employees within SMEs?

7) Can the employee OI process be influenced by customising training interventions within the organization?

The questions set out above were designed to encompass the real nature of the respondents' perceptions or interpretations of their holistic experience, and 
exploration of the depth of these meanings or interpretations in itself requires a reflective analytic approach, with phenomenological method considered the most suitable (Van Manen, 2007), hence the choice of IPA.

\subsubsection{Sample Selection}

According to Smith, Flowers and Larkin (2009, p. 48), sampling must be theoretically consistent with the qualitative paradigm in general, and with the IPA's orientation in particular.

This is consistent with Silverman's (2006, p. 306) rationale that sampling in qualitative research is neither statistical nor personal: it should be theoretically grounded. Therefore, for this research, the theoretical grounding for the sampling is based on the 'purposive sampling' method where the sample comprises individuals who are best placed to answer the research questions (Saunders, Lewis \& Thornhill, 2012).

SMEs are the main focus of interest as they account for $99.3 \%$ of all private sector employment in the UK and a combined annual turnover of $£ 1.9$ trillion, which is $51 \%$ of all private sector turnover (DBEIS, 2017); hence they are an important site of research.

\subsubsection{Research Respondents}

Qualitative researchers often use non-probability samples in selecting the population for their study. In which case such non-probability samples are deliberately chosen to mirror or reflect particular features or characteristics the researcher(s') is interested in from groups within the target population. These samples are not intended to be statistically representative, but rather exhibit attributes of the relevant population within a defined selection frame. Hence, non-probability sampling is considered to be well suited to small-scale, in-depth studies.

One such example of non-probability sampling method suited for qualitative inquiries is purposive sampling, which is the approach employed in this research to define the 
sample of the research population used. Purposive sampling approach dictates that the selection of participants, settings or other sampling units is based on a set of clearly defined or purposive criteria (Mason, 2002; Patton, 2002). Thus, samples are chosen based on certain clearly defined attributes or characteristics they exhibit which enable an in-depth exploration and understanding of the central themes under study or scrutiny.

Such defined characteristics may be generic socio-demographic characteristics, or sometimes more specified experiences, behaviours, roles, attitudes are also described as judgement-based (Burgess,1984 and Honigmann,1982 both cited in Ritchie, Lewis \& Elam, 2003). It is also sometimes called a criterion-based method, with LeCompte \& Preissle (1993) maintaining that this is a more fit term because all sampling methods have an element of purposiveness, but purposive sampling is still the widely used term in most academic literature.

In purposive sampling members of a sample are chosen with a specific 'purpose' to represent a location or attribute relative to a key criterion or subject matter under discuss. This firstly helps in ensuring that all key components, features or aspects of relevance to the subject matter under discuss are covered.

In addition, this 'purposive' nature of the sampling method helps to ensure that, some diversity is included within each of the specified criteria to aid exploration of impact of such criterion or characteristic. As such for instance, age is often commonly used as a selection criterion. This is particularly crucial in ensuring that that all relevant age groups are included in the sample size, as well as to facilitate exploration of any inherent differences that may be present in the perspectives of respondents within the varied age groupings explored.

When using purposive sampling method, decisions about which criteria are used for selection are often made at the early stages of designing the research. As such the choice of criteria is informed by a ranges of factors such as: key aims of the study; extant knowledge or existing theories within the field of study; hypotheses that the researcher(s) may want to explore; gaps in knowledge about the study population; and nuance the study intends to contribute to the extant body of research, in the field 
of study under scrutiny. Also, if on conducting the fieldwork, additional or supplementary samples are indicated or needed, then these can also be selected.

There are a diverse range of approaches used in purposive sampling, which are designed to yield different sample composition types often employed at the discretion of the researcher(s) and dependent on the intended aims and objectives of the research as well as coverage. Some of these are described as follows:

- Homogeneous samples (Holloway \& Wheeler, 1996; Patton, 2002; Robson, 2002) chosen to give a detailed picture of a particular phenomenon - for example, individuals belonging to the same subculture or have the same characteristics. This facilitates detailed investigation of social processes in a specified context.

- Heterogeneous samples (Holloway \& Wheeler, 1996; Robson, 2002) or maximum variation sampling (Patton, 2002). Here, a deliberate strategy to include phenomena which vary widely from each other is employed. The method aims at identifying central themes which cut across a variety of cases or people.

- Extreme or deviant case sampling (Patton, 2002; Robson, 2002). Samples are chosen because they are uncommon or special and therefore potentially more interesting or enlightening. The logic here is that a phenomena can be learnt more in-depth by looking at the exceptions or exploring its extremes (for instance, ethno-methodologists sometimes use deviant sampling to explore implicit assumptions and norms).

- Intensity sampling (Patton, 2002) this uses a similar logic to extreme or deviant case sampling but the focus here is on samples which strongly represent the phenomena of interest rather than uncommon or unusual samples. This however often require the researcher to do more exploratory ground work to determine which samples best implements intensely the subject matter under investigation.

- Typical case sampling is another method which chooses samples that are considered 'normal' or 'average' to provide detailed profiling (Patton, 2002). 
Selecting these sample size required prior knowledge of the overall patterns of response from the population on the subject under scrutiny such as in intensity sampling above, so that what is 'average' or 'typical' is known. For instance, participants might be selected from their responses to an initial survey.

- A hybrid approach called stratified purposive sampling can also be used. This approach aims at selecting samples that exhibit differences or variations in perspectives or behaviour on the particular phenomenon under scrutiny but with each sample groups also fairly homogeneous, so that effective comparison within subgroup samples can be done (Patton, 2002).

- In critical case sampling samples are chosen based on how they emphatically demonstrate a phenomenon or position 'dramatically' or are fundamental or important in the delivery of a process or operation (Patton, 2002). The rationale is that these samples are or will be 'critical' or 'integral' to any intended understanding offered by the research. This sampling method is particularly used in evaluative research as it helps focus attention to particular features of a process (Patton, 2002) and hence improve the potential impact of the research.

Other similar selection methods such as snowballing; theory-based; purposeful random; opportunistic or emergent; confirming or disconfirming case; as well as convenience technique all conforms to the ideology of purposive sampling method which stipulates adherence to a set of defined criteria for selecting a research sample from the given known or unknown population.

Thus, although 'purposive' sampling selection literally involves making deliberate choices, this does not in any case suggest the presence of bias in the nature of the choices made. As the use and implementation of purposive sampling requires substantial objectivity to ensure it meets independent scrutiny research standards. Thus even if the researcher or research funders have hypotheses on the phenomenon under study they want to test or understand, the opportunities for these to be proved or disproved need to be the same. 
Thus, following on from the procedural dictates of the IPA methodological approach employed within this research study, the research respondents were purposely selected across different sectors of the UK economy. The researcher also endeavoured to select research respondents to represent three geographically distant regions across the breadth of the UK (London; West Midlands; and North East), as well as six distinct sectors (Health and Social Care; Food; Financial; Information Technology (IT); Retail; and Education), to encourage diverse opinions and richness in the narratives of these respondents.

Most of the sectors chosen are able to conveniently showcase a unique perspective on the phenomena under study, although the IT and Healthcare sectors are more widely covered in OI literature (Smith et al., 2009). Thus, the research objectives and questions were significantly relevant to the circumstances of each selected research participant, which enabled collation of a detailed and comprehensive set of textural data for analysis.

The main factors considered in the choice of research respondents from the abovenamed sectors of the UK economy included the following:

- Firstly, the roles of employees in production or service delivery within SMEs in the researched sectors cannot be overemphasised, as these workforce are the main powerhouses and drivers of operational activities within these sectors. That is, these employees' roles are often integral to the activities within small business organizations. For example, within the education industry of the UK both academic and non-academic employees are crucial to the delivery of the main product sold to customers within the industry, i.e. 'education is sold to students within and outside the confines of the classrooms'. This means that a higher education institute cannot deliver its set targets or objectives related to providing education without its employee workforce.

This applies similarly across the other sectors such as health, financial, retail and the food industries; i.e. in the retail sector where most of the goods sold are homogenous in nature, provision of good service to customers by employees on behalf of the organization improves significantly the chances of having repeat 
customers, which brings in improved sales for the organization (Ferdous \& Polonsky, 2014).

Also, as human resources, engaged employees often act as a major performance indicator for organizations within these sectors. Their respective organizational performances and hence successes are often reflective of value customers perceive from services received, rendered by their workforce, and thus proportional to the employee performance levels evident (Farrell \& Oczkowski, 2012).

Hence, exploring this study's aim around understanding employees' Ol process within these sectors is relevant to the OI topic under study. Workforce (employee) performance levels have been also linked to employee OI levels displayed ( $\mathrm{He} \&$ Brown 2013). This is especially the case for most SMEs, which due to their smaller numbers of employees and lower financial capability are often heavily reliant on manpower rather than machinery to carry out most operational activities (Lee \& McGuiggan, 2008; Valadez, 2011).

It can however be also argued that the importance of employees within these six sectors may vary. For instance, while in educational organizations the success of the company mainly rides on the education service delivered by the tutors, within the retail industry the products offered by the company can be sold to consumers based on its standalone brand image without the input of employee services, as in the case of online shopping.

- Secondly, in terms of contributing to the academic body of literature within the research field, while OI has been explored in various academic literatures within other economic sectors such as such as IT engineering and, predominantly, health sciences (Bacaksiz, Tuna \& Seren 2017; Carlin, End \& Mullins, 2010), there is a significant paucity of literature providing evidence of the Ol construct within SMEs in other sectors explored in this research, such as food and finance.

A few Ol literature studies have explored these sectors, such as Farrell and Oczkowski (2012) in their exploration of the relationship between $\mathrm{OI}$ and 
organizational citizenship behaviour within employees in the service industry and Kim et. al. (2010) who examined the impact of students' identification with a university on intentions to support the university, establishing a pattern of events between $\mathrm{Ol}$ and academic departments in HEI. Also, a link between $\mathrm{Ol}$ and knowledge management was established by Cardoso, Meireles, Moreira and Matos (2010) as well as by Tsui and Ngo (2015) who examined the effect of four organizational antecedents on $\mathrm{Ol}$ among faculty members of a Hong Kong business school within the Chinese context.

Nevertheless, none of these highlighted literature studies or any others, to the best of my knowledge, have explored the impact of training as a dependent variable on the notion of $\mathrm{Ol}$ and how this is construed within small businesses. Exploring these mentioned sectors relative to the OI process will help jump-start research to map the influence of training as a strategic HRD practice on the employee OI process, which is essential in ensuring positive strategic alignment of organizational objectives and people policies as well as facilitating strong competitive advantage (Gratton et al. 1999). In addition to this, this research will facilitate the availability of training influence on $\mathrm{Ol}$ related literature references on which future research work can be based in the furtherance of OI research studies.

- A third rationale for choice of these sectors is their economic benefits. In statistical terms, with SMEs accounting for at least $99 \%$ of the businesses in every main industry sector, the six sectors chosen accounts for a $34.3 \%$ share of total SMEs population in the UK as well as $41 \%$ of their employment figures collectively, thus making the dominant contribution of approximately $46.5 \%$ of UK SMEs' turnover (DBIS, 2016).

\subsubsection{Sample Size}

A fairly homogenous sample is often required when using the IPA method for research (Correia, Santos \& Passmore, 2016), which means that utilising purposive sampling is a significant and relevant method for defining the sample respondents. This homogeneity also enables further studies to be carried out within other 
homogenous groups and a gradual evolution into the ability to make generic claims about research outcomes (Smith et al., 2009). Thus, a sample size of one can be adequate in some contexts, although in general, most IPA studies have tended to use between five and fourteen participants/data sets (Correia, Santos \& Passmore, 2016; Hutagalung, 2016; Singh, 2016; Smith et al., 2009)

Thus, using the figures iterated above as a guide, a total of fifteen SME employees were interviewed, with each interview session lasting approximately an average of about 60-180 minutes. These organizations, averaging 10 to 55 employees, fall within the small business category of SMEs (DBIS, 2016). For all fifteen (15) respondents, as seen in table 2 (p.117), the names have been falsified in accordance with relevant research data collection technique confidentiality and disclosure dictates, as well as the relevant University of Gloucestershire code of research ethics.

Table 2 below (p.117) describes the demographic distribution of the research respondents; their length of employment in current small business organisation; their respective positions within the organisation that is their various job roles; each respondent's designated industrial sector at the time of conducting the interviews; as well as indicating job skill levels such as whether the respondents are highly skilled professionals or low-skilled employees within their various SMEs and sectors. The actual individual interview durations are also indicated in the set of information included within table 2.

In resonance with the rationale for choice of diverse sectors stated earlier in section 3.6.2.1(pp.112-114), participants were drawn from different sectors of the UK economy in order to characterise the Ol process according to a range of participants' perceptions and experiences, especially in relation to organizational training interventions. Table 2 illustrates these variety in terms of sectoral distribution (DBIS, 2016). 


\subsection{Sample Selection Criteria}

As the study is employing a purposive sampling method, the set of selection criteria needs to be clearly defined (Patton, 2002). Thus, in summary there were three main inclusion criteria for participation in this study which are listed below:

a) Interview respondents must be SME employees, as this is the primary study niche of the research;

b) Interview respondents must be resident in the UK which is the geographical context of the research; and

c) Interview respondents must be within the age range of late teens to $60 \mathrm{~s}$. This last criterion was mainly used to capture viable experiential data reflecting an average of at least one to two years' working experience, as the adult age in the UK is 18 years, with the exception of Scotland (16). Inclusion of this criterion makes the data on the respondents' experiences and perceptions more comprehensive and therefore enhances the research results' reliability.

The entire interview process as per pre-arranged meetings was completed within a four week duration. This gave the researcher ample time to reflectively work through each of the interview transcripts individually before conducting the collective analysis. Further procedural techniques employed during the interview process is discussed in more detail within the data collation procedure sections 3.6.4 and 3.7 (see pp.119 to 128).

All respondents were briefed about the research intent and gave full consent informally prior to the scheduled interview dates. This was further consolidated by means of a participant information sheet distributed on the interview dates as well as signed consent forms (samples of which are attached in the thesis appendices). A more detailed section on ethical considerations will explore the relevance of these documents later in this chapter (see section 3.8, see pp. 128 to 130). 
Table 2 - Demographic Distribution of Research Respondents

\begin{tabular}{|c|c|c|c|c|c|c|c|c|}
\hline S/No & Name & $\begin{array}{c}\text { Age } \\
\text { (years) }\end{array}$ & Gender & $\begin{array}{c}\text { Length in } \\
\text { current } \\
\text { SME } \\
\text { (years) }\end{array}$ & $\begin{array}{c}\text { Position within current } \\
\text { SME }\end{array}$ & $\begin{array}{l}\text { Job } \\
\text { Skill }\end{array}$ & Sector & $\begin{array}{c}\text { Interview } \\
\text { Timings } \\
\text { (Mins) }\end{array}$ \\
\hline 1 & Tanya & 41 & Female & 2 & Assistant Sales Manager & Low & Retail & 168 \\
\hline 2 & Tracy & 28 & Female & 3 & Accounts Clerk & High & Financial & 95 \\
\hline 3 & Wendy & 39 & Female & 2 & Administrator & Low & Education & 138 \\
\hline 4 & Rose & 41 & Female & 5 & Senior HCA & Low & Health and Social Care & 88 \\
\hline 5 & Gilbert & 40 & Male & 2 & Senior IT Project Analyst & High & IT & 180 \\
\hline 6 & Rosemary & 27 & Female & 2 & Learning Disability Assistant & Low & Health and Social Care & 65 \\
\hline 7 & Binta & 29 & Female & 1 & Sales Assistant & Low & Retail & 129 \\
\hline 8 & Ben & 29 & Male & 1 & Health Care Assistant & Low & Health and Social Care & 127 \\
\hline 9 & Debra & 39 & Female & 1 & GP & High & Health and Social Care & 89 \\
\hline 10 & Patricia & 37 & Female & 2 & GP & High & Health and Social Care & 65 \\
\hline 11 & Timothy & 19 & Male & 1 & Waiter & Low & Food & 198 \\
\hline 12 & Kyle & 19 & Male & 1 & Waiter & Low & Food & 126 \\
\hline 13 & Frederick & 60 & Male & 4 & Health Care Assistant & Low & Health and Social Care & 154 \\
\hline 14 & Mandy & 20 & Female & 1 & Support Worker & Low & Health and Social Care & 113 \\
\hline 15 & Rena & 34 & Female & 1 & Dietician & High & Health and Social Care & \\
\hline
\end{tabular}




\subsubsection{Data collection Instrument}

Interview is the main instrument used for data collection within this research. Interviews, essentially "a conversation with a purpose" (Smith et al. 2009, p.57), are used when the nature of the research requires collection of more subjective and indepth data to make meaningful interpretations from the opinions of research participants (Bryman \& Bell, 2003; Easterby-Smith et al., 2008; White, 2002).

Within the IPA approach, use of interactive interviewing also helps both the interviewer and interviewee to be active participants within the research process (Singh, 2016). Thus, the interviewer(s) co-constructs the meaning attached to the experiences narrated by the interviewee(s), mainly from the interview transcripts but also during the actual interview session when probing for more clarity and information from the respondents about the subject matter being researched (Singh, 2016). Meaning co-construction facilitates the interviewer's understanding of the subject matter under scrutiny by juxtaposing the understanding of the interview vis a vis the experience.

The face-to-face interviews conducted followed the semi-structured format, which combines the flexibility of the unstructured interview with the controllability of the structured interview to allow fluidity in discussion (Fisher, 2004; Flick, 2009; White, 2002; Hutagalung, 2016). The semi-structured interview, being a flexible data collection instrument, enables researcher and respondents to engage in dialogues wherein questions pre-emptively compiled are modified to reflect the direction of the respondents' narratives (Smith \& Osborn, 2009, p.105 cited in Hutagalung, 2016). The questions asked also tend to be open-ended and exploratory in nature (Smith et al., 2009).

Fisher et al. (2002), however, noted that the technique is unavoidably prone to individual bias given the subjectivity of the research. It is nevertheless justified for 
use within this thesis based on the authenticity, trustworthiness and revelational attributes of the study's IPA methodological stance that enhance understanding the experiences of the study respondents (Guba \& Lincoln, 1994). The semi-structured interviews also had the advantage of being sufficiently flexible to enable the participants to explore issues in depth and at their own pace.

\subsubsection{Process}

This sub-section discusses the timings, location and all other additional considerations relating to the research interview sessions conducted.

The timing of the data collection was dictated by the nature of the research questions; the feasible and realistic availability of the participants, especially in terms of the pre-empted time needed for the interviews; and the completion deadline for the research study.

Using the research objectives as well as the Mael and Ashforth (1992) Ol six-item scale comprising questions such as "The successes of (organization name) are my successes" and "When I speak about (organization name), I usually refer to 'we' and not 'they", and the Podnar, Golob and Jancic 2011 scale with questions including: "I feel that the company I work for is a part of me" and "I enjoy interacting with my coworkers" as a guide, the number of structured thematic research questions known as SRQs (see section 3.6, pp. 103 to 109) pre-compiled for the interview was limited to seven (7), apart from those in the general demographic information section. This also enabled better fluidity in the exploration of the OI phenomenon under study by probing the respondents and facilitating other organically generated questions during the interview process. 
Moreover, as the idea of IPA is to 'gather information for the research question by approaching it 'sideways' (Smith et al., 2009, p. 58), that is, indirectly, the interview questions needed to be broadly framed to facilitate a dialogue. Hence, the set interview questions were designed to be suitably and descriptively specific, but also open-ended and general enough to encourage the participants to offer their own perspectives on Ol (Smith et al, 2009), as well as to facilitate the interviewer's indepth understanding of these perspectives. Thus, even though IPA advocates that participants should be free of control, each participant was steered to respond within the broad framework of the main posed questions.

The interview questioning was overall focused on three key areas addressing the research objectives: Understanding of Ol; Identifying employee OI markers in the organization, including training; and exploring the influence of training on the employee OI framework. This thematic categorisation were based on seven (7) core $S R Q s$, which were then deconstructed to formulate further probing questions and prompts to facilitate a deeper understanding. A few of the sample questions are highlighted below, with the detailed list included in appendix I section of this thesis:

- When you hear people say "I love what I do" or "I love my job" what comes to mind?

- In one sentence, how would you characterise how you feel when at work? Could you feel better or worse than you feel now?

- Do you think you are suited to your current organization? Can you please elaborate on this?

- $\quad$ Do you think of your organization as an identity?

- Would you say your own identity matches that of the organization? How? and why? 
The interviews took place in a neutral environment, outside the respondents' workplace confines, to limit any probable associated stress or pressure that might have been caused if they had taken place within the interview respondents' organization itself. This enabled the participants to be more relaxed in voicing their individual narratives and stories in relation to the subject of Ol using open-ended questions (Smith \& Dunsworth, 2003).

The interviews, which lasted from a range of 60-180 minutes for each respondent, essentially took place within a period of four weeks. The interviews also took place after the respondents had given verbal consent prior to partaking in the research. A documental package of research context, instructions, consent forms and pre-set interview questions had been electronically sent out to each respondent after which on receiving consent, the interview sessions were scheduled with individual respondents. This was further reinforced with physical copies of the consent forms administered before the commencement of the questioning. Also, to further ensure that respondents consent was a continual process, participants were informed that they could choose to withdraw from the research at any time prior to the completion of the data analysis.

Finally, all the interview sessions were audio-recorded and then transcribed verbatim in adherence to the IPA procedural approach which is discussed in more depth in the subsequent sub-section 3.7 .

\subsection{Data Analysis Procedure}

The quality criteria for qualitative research, such as sensitivity to context, commitment and rigour, transparency, coherence and impact and importance (Yardley, 2000), were all crucial and kept pertinent in the researcher's mind throughout the data analysis stage. 
The interview transcripts were transcribed verbatim and then reflectively interpreted to understand the underlying markers of respondents' Ol process. This aligns with the proposition by Van Manen (2007) and Smith et al. (2009) that phenomenology, and more appropriately IPA, needs to follow an 'empathic' approach where the researcher tries to understand what it is like from the perspective of the respondents through probing questions asked during the interview sessions and thus the interview transcripts later generated which aimed at unearthing what lies beyond the obvious, thereby enacting a process of reflexivity.

'Lived experiences gather hermeneutic significance as we reflectively gather them by giving memory to them' (Van Manen, 2007, p. 7). That is, the aim of phenomenology is 'to transform lived experience into a textual expression of its essence, in such a way that the effect of the text is at once a reflexive re-living and a reflective appropriation of something meaningful: a notion by which a reader is powerfully animated in his or her own lived experience' (Van Manen, 2007, p. 36).

Smith, Flowers and Larkin (2009) advocate that IPA analysis needs to follow an 'empathic hermeneutic' approach whereby the researcher tries to understand what it is like from the point of view of the participant and using 'questioning hermeneutics' by asking in-depth questions from the interview transcripts aimed at uncovering what lies beyond the obvious. Both aspects of the inquiry are likely to lead to a richer analysis and to do greater justice to the totality of the person.

Thus, IPA provides guidelines that can be adapted by various researchers to their unique research aims (Smith \& Osborn, 2003), helping the researcher to analyse data either by following steps recommended by Smith et al. (2009) or by incorporating additional stages, or by using their own analysis framework.

Furthermore, by its nature, qualitative research dictates that there is a great deal of subjectivity both on the part of the participant and the researcher which, according to 
Parker (2005), enables a more personal phenomenological approach to investigation than is possible with quantitative research. Therefore, the analysis of data also significantly involves the practice of reflexivity by interpretation of the data by the researcher while bracketing own experiences.

Therefore, having read a series of IPA related texts (Biggerstaff \& Thompson, 2008; Chapman \& Smith, 2002; Correia, Santos \& Passmore, 2016; Singh, 2016), the following data analysis procedural steps was followed:

a) An initial reading of interview transcripts which enabled better understanding of narrated emergent themes:

The researcher read through each of the interview transcripts to get the general tone and perceived understanding of each interviewee's view on the Ol process in general. Responses to questions were then cross-referenced with the interviewer's notes and connotations scribbled down at various intervals during the interview recording. These included noticed physical gestures and expressions of the respondents while narrating. This phase of the analysis procedure aimed to clarify the research objective one (1) to explore the understanding of employee organizational identification within SMEs'. The understanding and interpretation derived from the in-depth read of each interviewee's transcript in relation to the others helped to generate sufficient insight in ascertaining the consensual view of the employee OI process among the research respondents.

b) Highlighting and definition of holistic emergent themes across transcript narratives. This helped give structure to aid the discussion of findings: 
Here, the researcher took time to identify and note down various conceptual points that defined the understanding of the OI process, employer situationalcontext and employee situational-gap OI markers within the context of each respondent's interview narrative. These were then compared to other interviewee's identified components and a comprehensive list of these Ol markers was generated from the 15 transcripts. This step helped address research objective (2): 'to ascertain the markers of employee organizational identification in SMEs'.

c) Cross referencing the emergent themes to elements of the proposed $\mathrm{OI}$ framework to validate the role and appropriateness of each element within the framework:

Within this phase, the researcher cross-referenced the emergent markers with those pre-empted in the initial conceptual research framework and in essence reconstructed the framework to mirror the resulting themes from the data analysis. This stage helped address research objective three (3): 'to explore how training interventions influence the employee organizational identification process'. This stage aided the use of the psychological practices of reflexivity and reflections by interconnecting the various elements from the emerged themes to the conceptual themes and vice versa, thus helping to elicit grounded themes and abstractions (Smith et al., 2009).

d) Resulting themes deconstructed to form workable sub-sections under which the research findings can then be discussed:

Once the previous step was completed, completion of the current step became much more realistically feasible. As the various themes had been 
established, much of the work within this step was primarily structural and articulatory in nature. Herein the structural formation of this thesis began to take form and develop into a concrete seamless narrative

e) The final step involved a cross-case analysis of the fifteen interviews to ascertain the likely outcome of the OI process for each respondent, i.e. either $E O I$ or $E O D$ is formed. The researcher explored further to ascertain whether or not each of the Ol formed had a scalar magnitude or value. That is, whether there was a degree as to how the two possible Ol outcomes were expressed by the individual respondents. Also, time-permitting the researcher hoped to explore if this degree varied according to the sector each respondent worked in. While this was the intention, due to time constraints, I was only able to briefly explore the potential differences between respondents with high and low skilled jobs regardless of the industry or sector they worked in.

\subsubsection{Quality and Methodological Rigour of Data Analysis}

The level of quality and rigour needed in research work has been debated for a while in the academic field (Denzin \& Lincoln, 2000; Lincoln \& Guba, 1985; Kohler, Landis \& Cortina, 2017). Thus, in recent years qualitative rigour has been sought by proposing various ways forward for the researcher including: "rationally articulating a knowledgeable, theoretically informed choice regarding their approach to rigour and selecting an approach that is philosophically and methodologically consistent with their inquiry" (Caelli, Ray \& Mill, 2003, p.15).

Hence, in this IPA research study, as suggested by Smith, Flowers and Larkin (2009), I attempted to adhere to Yardley's four principles by seeking advice, feedback and support from other IPA researchers with whom I had been opportune to have contact. Yardley's four principles include: sensitivity to context; commitment 
and rigour, transparency and coherence; impact; and importance to demonstrate the rigour and integrity of the data presentation (Yardley, cited in Smith et al., 2009).

Also, Smith et al. (2009) suggested that IPA studies should aim to exceed the just 'good enough' cliché by evidently demonstrating commitment and rigour in the process. This means adhering to Smith et al.'s (2009) recommendation on the need for meticulousness and transparency at the data acquisition and analysis stages

To ensure transparency and integrity of the research process, I therefore sent a draft of their narrative interpretation to each respondent to read over, to ensure there was no misunderstanding of their perspectives and any identified were corrected before finalising the results discussion section of the thesis write-up. This connotes with Caelli, Ray and Mill's (2003) assertion that providing feedback to each participant enables them to consider the researcher's understanding and interpretation of their experiences alongside re-visiting their own articulation of their experiences.

Hence, this process of sending a written extract to each respondent enhanced the integrity of the research data analysis process. This is a recommended IPA practice and a way of authenticating the intent of the statements made at the interview (Singh, 2016). This is considered as a member check or 'participant feedback which is regarded as the single most critical technique for establishing credibility' (Guba \& Lincoln, 1989, cited in Rolfe, 2006).

However, this approach creates the possible threat of 'triple hermeneutics' where the research outcomes then become the participant's understanding of the researcher's understanding of the participant's experiences (Singh, 2016). I still chose to do this, time-permitting, as it significantly helped clarify any inherent misunderstandings in my interpretation of participants' experiences on the subject of $\mathrm{Ol}$ and the influence training has on their OI process and hence on their OI statuses as employees of their respective SMEs. Also, it encouraged an extra degree of participant scrutiny since 
IPA as an approach assumes that the participant owns the expert right to own experiences. It also combines the advantages of validity and accuracy of analysis within the subjective domain of qualitative research (Singh, 2016).

To buttress the evidence of rigour in this research further, in terms of the process's 'reflexivity, construction of texts that are credible to the experience and understood by insiders and outsiders' (Laverty, 2003, p. 23), the IPA approach employed aligns with my ontological and epistemological positions of relativism and interpretivism respectively through recognising the existence of many realities and its assumption that the eventual outcome of the researcher's study would have been informed by the varying perceptions or views of the respondents within the set organizational context (Easterby-Smith et al., 2012).

In addition to the view expressed above, my viewpoint as a researcher is in agreement with Finlay's $(2009$, p.17) notion that any knowledge produced is 'contingent, proportional, emergent and subject to alternative interpretations', as well as with Rolfe (2006) who argues that 'rather than search for an overarching set of criteria by which to judge the validity of qualitative research, we should perhaps acknowledge that there is a multiplicity of (so-called) qualitative paradigms, each requiring very different approaches to validity. There is no qualitative paradigm at all, so that each research methodology (and perhaps each individual study) must be appraised on its own merits' (Rolfe, 2006, p. 310), which is summarily a crucial importance of interpretivism as a variant of the relativism stance represented in research.

This mirrors my ontological position of 'many realities', as the primary aim and premise of my research is to acknowledge the 'reality' narrated by each SME employee respondent as being true from a phenomenological perspective, and then 
to construct my understanding as a researcher by identifying common emergent themes that occur across the entire participants' experiences.

In conducting qualitative research there is now a requirement for reflexivity as an integral element (Snape \& Spencer, 2003). There is also a growing belief in accepting and accommodating the researcher's perspectives, attitudes, motives and emotions during the duration of the research (Crabtree \& Miller, 1999; Langdridge, 2007). This acceptance of the credibility of the psychological input of the researcher is diametrically opposed to the traditional approach of objective and detached research, hence one more reason why the application of IPA methodology is apt in the context of my research.

Thus, a fairly detailed reflective account has been incorporated in the result presentation and analysis of results section of this thesis to reiterate the involvement of the researcher throughout the research process. The next sub-section turns to detailed discussion of the ethical issues considered and appropriated during the entire length of this research.

\subsection{Ethical Considerations}

"...Ethics involves morality, integrity...Morality is about knowing what is right and wrong, and integrity is about acting on that knowledge...Because we are human beings engaged in understanding other human beings-social realities- ethics is of the most utmost importance so that our research is not harmful" (Leavy 2017, p. 24).

According to Stutchbury and Fox (2009, p.489), 'all research undertaken in situations which involve people interacting with each other will have an ethical dimension... and the ethical issues are often complex'. Therefore, discussing the ethical issues 
considered throughout the length of the research is pertinent to ensuring the validity of this research thesis itself.

Research ethics refers to the moral standards, principles and procedural details guiding research from the start through to its completion, publication of results and beyond (ESRC REF, 2015). Since the audience of the research work must be able to trust the validity and integrity of the entire research process, I have included a detailed step-wise account of how the data was collated and analysed and describe in some detail how the research evolved into what was finally submitted for approval. In addition, the research data gathering process was transparent to the voluntary participants and they were made aware of the purpose for which their verbal account of experiences will be used during and after the thesis submission. This process is iterated in more detail in the earlier sections of this chapter (see pp. 108 to 128).

This research was guided throughout by the University of Gloucestershire's Research Ethics Handbook and Researcher Development Framework (2010), in order to adhere to rules on important research issues such as anonymity, data protection, confidentiality and approved consents from respondents. 'The Researcher Development Framework (RDF) is a major new approach to researcher development, to enhance our capacity to build the UK workforce, develop worldclass researchers and build our research base. The RDF is a professional development framework for planning, promoting and supporting the personal, professional and career development of researchers in higher education. It articulates the knowledge, behaviours and attributes of successful researchers and encourages them to realise their potential' (RDF, 2010 p.1).

It is significantly important to mention that ethical issues could arise at any stage of a research project. The ethical issues and possible dilemmas of researching in 
organizations are discussed within the Research Governance and Organization domain of the Researcher Development Framework (2010). This domain is concerned with responding to the ethical and legal requirements imposed by the research area. It informs professional conduct involved in the planning and completion of the research, addressing such as confidentiality matters, organizational accessibility approval and treatment of vulnerable groups in relation to carrying out the research study.

The ethical issues of confidentiality and data protection are crucial and need to be acknowledged in any study (Mason, 2002). Thus, all data gathering procedures, including audio-recording and then transcribing the interviews, were carried out in strict adherence to the University of Gloucestershire Research Degree Ethics Handbook and Code of Conduct. These recordings will be subsequently destroyed once the thesis has been approved. Also, the transcribed manuscripts anonymised participants' details such as names, workplace details and job titles to protect the research respondents' identity.

Sample of questions posed to the respondents and consent forms are also included in Appendix I of this thesis (see pp. 259 to 266).

\subsection{Research Governance and Organization}

"Holding membership in a group does not denote complete sameness within that group. Likewise, not being a member of a group does not denote complete difference... Furthermore, one does not have to be a member of the group being studied to appreciate and adequately represent the experience of the participants...It seems paradoxical, then, that we would endorse binary alternatives that unduly narrow the range of understanding and experience". (Dwyer \& Buckle, 2009, pp. 59-60) 
The positioning of the researcher as an insider or outsider is an important element of qualitative studies and there is the need to be consciously aware of one's position as a researcher from the onset to ensure ethical credibility and transparency in the collation and interpretation of data gathered (Dwyer and Buckle, 2009; Court and Abbas; 2013; West et al., 2013; Savvides et al., 2014).

As a researcher within this study my role is mostly from an 'outsider' perspective with some limited elements of insider perspective, having previously worked in small businesses (Refer to section 1.5.1, pp. 12 to 14). All the research respondents interviewed work in small businesses (a key defined criterion adhering to the purposive sampling nature of this research) in different industries from that of the researcher. Thus, by using a combination of multi-industry research participants, I was able to minimise any apparent insider disadvantage or bias that could have otherwise affected the outcome of the research.

Greene (2014) defines insider research as that which is conducted within a social group, organization or culture of which the researcher is also a member' (Greene, 2014, p.1). Insider research, often also called action research (Barnett, 2014; Coghlan, Shani \& Roth, 2016), includes various commonalities, such as language, race, history, similar experiences, religion, between the participants and researcher (Court \& Abbas, 2013; West et al., 2013).

Though research is often undertaken in the researcher's own work organization, this could have both advantageous and unhelpful implications. Some of these advantages and disadvantages have been articulated in several studies (Dwyer \& Buckle, 2009; Wainwright \& Sambrook, 2010; Bhatta, 2013; Court \& Abbas, 2013; West et al., 2013; Wilkes \& Halcomb, 2013; Savvides et al., 2014; Greene, 2014).

A common theme across all these studies is the recognition that insider research has a legitimate place in qualitative research, as such research often results in rich data 
and empathic interpretation (Dwyer \& Buckle, 2009) due to the close involvement of the respondent and researcher. Other highlighted benefits include: acceptance, access to participants; openness; better trust-relationship; subjective knowledge of the subject of inquiry as well as the organization under study; and less power differential between the researcher and participants as both are members of the same organization (Dwyer \& Buckle, 2009; West et al., 2012; Hanson, 2013; Wilkes \& Halcomb, 2013; Singh 2016).

However, the insider researcher is often exposed to difficult questions about research objectivity, reflexivity, and authenticity of a research work because the researcher may be said to know too much or be too close to the project and possibly too similar to those being studied (Kanuha, 2000, cited in Dywer \& Buckle, 2009).

Other associated disadvantages may include their shared background resulting in participants assuming the researcher should understand implied meanings which have not been voiced. Additionally, there could be complacency in probing, superficial analysis and biased interpretation of data, while juggling the conflicting demands stemming from the dual identity of researcher may result in power imbalances because of the authority relationship of the researcher-respondent within the organizational structure (Wainwright and Sambrook, 2010; Savvides et al., 2014). Also, as noted in Brannick \& Coghlan, (2007), researchers might struggle with role conflict if they find themselves caught between 'loyalty tugs' and 'behavioral claims'.

A mostly outsider perspective reinforced with elements of an insider perspective based on previous work experience in similar SMEs places the researcher in a good and fair position, as she was able to circumvent the disadvantages of being too close to the research as an organization insider, but drew on her previous work experience in similar sized SMEs to inform the conduct of this research. Also these elements of 
an insider perspective enhanced the depth and breadth of her understanding of the research population.

Ease of access could pose another major challenge, as could gaining the required level of trust to enable the participants to share their experiences truthfully. However, as I was mainly an outsider researcher, the respondents felt quite at ease and unencumbered in providing more detailed and explicit accounts of their work experiences and stories.

I also ensured special care was taken to bracket my own assumptions when trying to interpret the interview transcripts to avoid bias and as a researcher I was able to develop a fairly good rapport with the respondents prior to the scheduled interview sessions so that they were able to relay their experiences in an informal and more genuine way due to the level of trust already existing between the researcherrespondents (Hanson, 2013; Savvides et al., 2014).

Despite these highlighted issues, as iterated by Dwyer and Buckle (2009) 'the core ingredient is not insider or outsider status but an ability to be open, authentic, honest, deeply interested in the experience of one's research participants...' I was committed to undertaking a credible research that mirrors good values such as integrity, validity and transparency, which to the best of my knowledge I have been able to do, even by acknowledging the possibility that I could be a source of error in the research process. I believe that I have thus been able to demonstrate the attributes listed by Dwyer and Buckle (2009) as necessary to provide an accurate and adequate analysis and discussion of my respondents' experiences, as well as a detailed reflection on the process while exhibiting disciplined bracketing skills. 


\subsection{Conclusion}

This chapter explored the various underlying philosophical and methodological stances underpinning this research study, as well as narrating in detail the step-wise sequence employed in the research design and data analysis.

The ideologies and rationale elucidated in this section are applied to guide the narrative in the subsequent chapter four of my thesis to ensure the validity and integrity of the entire research process. Each highlighted step is then followed meticulously and enacted to improve the reliability of the research results and outcomes in making more generalisable conclusions on the research subject of OI. 


\section{CHAPTER 4 \\ Research Findings, Analysis and Discussion}

“... Inquiry can be enhanced if different views-of-the-world are used to inform each other. In an exploratory field study...sense-making assumes ...action is a continuous, equivocal stream of experience that can only be understood (or made sense of) when it is viewed in retrospect". (Boland Jr. 1984, p. 868)

\subsection{Introduction}

The current chapter presents the primary research findings, including excerpts from the initial pilot study conducted, and in-depth analysis and discussion of the research findings using the research design framework (RDF) previously introduced in the methodology chapter of this thesis.

Based on the incorporated reported experiences of the research respondents regarding the impact of organizational identification markers, including training, on their organizational identification process within an SME context in the UK, this chapter critically details the presentation, analysis and discussion of these findings in six main sections. The first summarises the data analysis section for the initial pilot study; the second section gives a summary of demographic information related to the interview respondents. A third section gives a descriptive overview of the research respondents' stories.

In the fourth section, findings are presented based on each of the primary RQs addressed by this exploratory research. The discussion of findings are crossreferenced with relevant extant literature related to the OI construct under scrutiny. The key analysis section (section 4.4, pp. 150 to 194) is split into three sub-sections 
addressing the three essential sections within the conceptual research framework (CRF). The discussion of findings follows the pathway evident in the RDF presented, at the same time linking the CRF sub sections with the RQs primarily explored by this thesis. It follows a step-wise thematic perspective adapted from IPA methodology which helps to ensure procedural and contextual relevance to the organizational identification concept under discussion as well as to the context of interview respondents' stories presented within this study. This section 4.4( pp. 150194) also presents an in-depth analysis of results from the interview SRQs, pregenerated from the RQs, and discusses how each $S R Q$ contributes to addressing the relevant $R Q$ depicted by the CRF components.

The fifth and six sections briefly details outcomes of analysis on the impact of sectoral differences and job skill influence on employee Ol outcomes respectively (sections 4.5 \& 4.6, p. 194), while an additional final seventh section summarises key points highlighted in the entire chapter 4 of the thesis.

\subsection{Presentation and Analysis of Pilot Study Findings}

Section 3.5 .1 (p. 101) details the categorisation of the initial pilot data themes into five key categories namely:

- How Training is defined;

- Typologies of Organizational Trainings;

- Training receptivity pre-, during- and post-training activities;

- Motivation for Training;

- Post-training use and cost.

In category one, all five respondents mutually defined training as facilitating the learning process relevant for their professional development. It is seen as a 
technique for increasing knowledge to improve professional competence, as well as a set of learning programmes to meet current work environment demands in adherence to organizational policy. This notion about training, focused primarily on learning initiated by their respective companies (section 4.4.3.1, pp. 190 to 192), was also evident in the main study outcomes; however, an additional dimension to training termed 'facilitated training' as seen in the revised CRF figure 9 (p. 203) and explained in depth in sections 5.4 and 5.5 (pp. 204 to 213) was conspicuously absent from the narratives of the pilot study respondents.

In tandem with the results of the main study, initiated training activities acted as an employer situational-context marker for employees' OI status, wherein respondents affirmed it as a good predictor of how they identify with their organization, especially when the initiated training offered is in sync with the requirements of their professional competence development.

Nevertheless, they also highlighted that some initiated training activities offered within their companies were merely offered to comply with organizational policies and as such do not have the same desired identification effect on their perception of belongingness (OI statuses) with the organization.

A second categorisation of the pilot study results indicated employees' perceptions and understanding of the varying types of training activities implemented within their small business organizations. These typologies included forced or mandatory training programmes; training activities tailored for organizational compliance; seen in figure 7 (p. 142) as well as the training activities perceived as most useful which are those that help to enhance the employee's professional development and personal career aspirations.

The pilot study respondents' perceptions of organizational training activities were mostly rather distrustful as they were considered mainly reactive to governmental 
expectations or requirements rather than for employees' benefit. Thus, these perceptions often translate to them not benefitting substantially from such organised training activities, as they are not often receptive about what is being taught as a result of the prior preconceived opinions they have of the rationale for these activities.

In comparison to the pilot study, in the lived-experiences narrated by the main research respondents, most of the training typologies identified by the employees were in agreement. Examples such as in-house trainings tailored at ensuring organizational compliance to industry and governmental rules and regulations (attendance at such for example health and safety, data protection training seen in section 4.4.3.1, pp. 190 to 192 were also mandatory); also, e-trainings that could be accessed remotely by the trainees and thus can be completed on- or off-the-job as well as trainings organised by the company which simultaneously addressed some of the employee's continuous professional development(CPD) needs were highlighted.

A new training type, however, emphasised in the main research but not in the pilot study is the facilitated training identified in section 4.4.3.2 (pp. 192 to 194). As explained, facilitated training refers to training activities undertaken by the SME employees that are not organised by their companies, but they support them by providing good compensatory incentives to acknowledge their commitment to such training, which is often tailored to the individual employees' CPD.

In general, the main study respondents' underlying perceptions of training activities initiated by their organizations were mostly positive. For example, while some trainings organised by the company were mandatory and for compliance purposes, the respondents did not regard these as mere platitudes or patronising but rather felt 
the training activities were quite important in performing their everyday duties effectively and efficiently and in reducing occurrence of accidents.

However, a key problem identified by the main research respondents in contrast to the pilot respondents was the absence of sufficient training activities offered by their companies to cope with the demands of their role within the company in essence.

The third category highlighted the trainees' perception of training receptivity in the pre- during- and post-training stages. The first pre-training stage was determined by a combination of factors including the purpose of training, timing of training, mode of training activities and whether or not employee's participation was mandatory or forced as used verbatim in the interview narratives. Employees accredited their perception during-training mostly to how useful they perceived the training content was to their individualised Job roles, training delivery, timing of training (including time length) and their personalised and often developmental need for a specified training course. In the third post-training stage, the trainees' perception was also influenced by the usefulness of training activities to their individualised Job roles, ease of application on-the-job, employer's directives on use of training contents and their overall job satisfaction.

Comparing these perceptions with the main study narratives, while the main respondents did not emphasise the demarcation of the training stages in relaying their perceptions, cogent points like relevant timing of the training, importance of contents to individual job roles, overall job satisfaction and how well these activities meet their CPD needs were identified as facilitatory to development of EOI statuses rather than EOD (sections 4.4.1.3 to 4.4.1.5, pp. 154 to 163 ).

The fourth category explored factors driving employee's motivation for training. This was sub-divided into two sections, namely Personal and Professional, such as better work-life balance, better financial security for family, professional certification(s) and 
Organizational such as intra-company promotions, forced or Mandatory training activities. Employees expressed that their motivation for training is hugely circumvented when these aforementioned factors are not met. Interpreting these in comparison to the main study outcomes, it could be closely linked to the finding that when these factors are not present EOD would often be predominant within such small businesses; thus corroborating most of the literature references related to the importance of training in organizations(Roy\& Raymond 2008; Sahinidis \& Bouris 2008).

The last category focused on post-training use and cost. Pilot interviewees suggested that more often than not, training activities' outcomes do not justify the resources invested into such activities by the initiators and participants (that is the organizations and the individual employees respectively), mainly due to misalignment in the training design. That is, training activities are often generalised and not sufficiently tailored to the specific needs of relevant employees within small organizations when organised by the companies. Similarly, if employees decide to embark on a specific training activity on their own (initiated training), the cost of taking time off for such endeavours can often be expensive in different ways, such as financial cost and their job security, thus dissuading most employees from pursuing such training opportunities.

Nonetheless, in contrast to the above, main study respondents view their employers as being quite supportive of their exploring own-initiated training ventures that contribute to their overall professional capability and usefulness (section 4.4.3.2, pp. 192 to194). Employees are encouraged by giving them incentives such as time off in lieu as well as opportunities to influence their other colleagues positively with useful tools learnt in such trainings. 
In summary, outcomes from the pilot study suggested that employees would only tend to identify or have substantial belongingness $(\mathrm{OI})$ with organizations with which they share similar characteristics or if they admire the way in which organizational activities are handled in-company, thereby achieving the strategic fit as advocated by Gratton et al. (1999). Hence, the pilot study outcomes substantially advocate that small businesses can potentially influence EOI if they develop and implement organizational traits admired by their employees.

\subsubsection{Further Pilot Analysis: Employees' Perception versus Organisational Identification CRF}

The pilot study was designed to holistically explore the overall understanding of OI among employees within SMEs in order to identify relevant factors that possibly influence OI levels among employees in an SME context and whether training influences Ol dynamics within such organizations. This could seemingly be conceived as a regulatory tool businesses could employ in managing employees effectively.

Thus, furthering the analysis from the previous sub-section 4.1, figure 7 below depicts a summary of the pilot study showing the relationship structure of variables identified within the pilot study themes.

Deconstructing the various factors highlighted in figure 7 above reveals that employees who participate in training activities within organizations have somewhat malleable experiences or perceptions, which organizations can take advantage of to influence the OI levels exhibited by their employees. The next sub-sections further analyse the pilot outcomes by cross-referencing these with sense-making theories employed in my main research and the IPA approach using some wordings verbalised by respondents. 


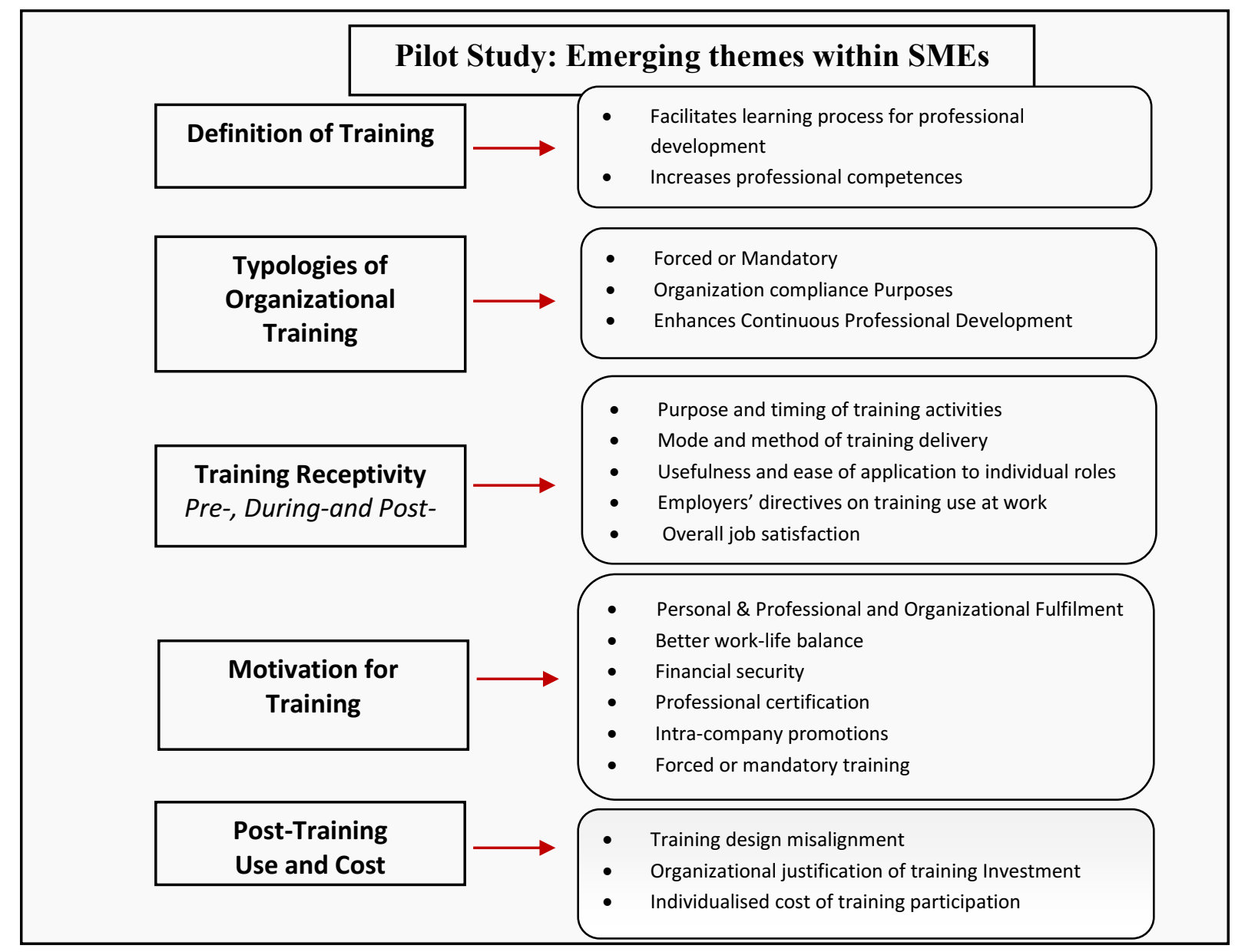

Figure 7: Pilot Study Emergent Themes 


\subsubsection{Force or Coercion versus Organizational Control}

One of the main typologies of training that constantly reoccurs within the sensemaking perceptions of the pilot employees regarding training activities is the notion of forced training (figure 7, p. 142). It emerges as a common notion that employers tend to organise training programmes, occasionally or intermittently, as a form of institutional compliance to be mandatorily attended by employees whether or not they want to and depending on the structure and stability of the organization's people management and development goals.

Thus, sharing their lived experiences, employees' sense-making of most training activities tends to be more along the lines of them being a "chore" to be done by the employee rather than being seen as a developmental opportunity. This thus correlates with the notion that training itself could be a sort of Identity regulator to influence employees individually within organizations.

\subsubsection{Power versus Self Efficacy}

Another factor highlighted by employees is that of fear of job insecurity. Respondents highlighted that if they decide to leave their jobs in preference to undergoing external training activities or when not happy with the training activities initiated by their current employers, they are faced with the possible occurrence of losing their jobs which often provide the source of livelihood for themselves and arguably their families.

In-depth scrutiny into this factor suggests that organizations, either intentionally or without necessarily realising, wield a substantial amount control or power over their employees' actions or inactions. Such may be the case when employees unwillingly consent to undertake organization-initiated training activities even when they do not initially have any significant personal interest or motivation to do so. This often unintended control then acts as a barrier to self-efficacy and mostly generates a 
sense of fear in the minds of the employees. This fear thus significantly contributes to the development of EOD within the sense-making process of the individual.

\subsubsection{Perception Variability over Time}

As was evident from the narratives of the pilot respondents, there were significant elements of time variability, particularly in relation to the perceptions of the trainees across the pre-, during- and post-training stages. Some of the employees' narrated lived experiences suggested that their perceptions can indeed change in support of the notion that employees' sense of belongingness (OI status) can change over time, either positively or negatively, as influenced by activities initiated by their organization. This corroborates the vector attribute proposed in the final chapter of this thesis asserting the notion that $\mathrm{Ol}$ has both directional and magnitudinal attributes.

\subsubsection{A Sacrificial Opportunity Cost and Perseverance}

Another factor evident in the pilot respondents' verbal narratives was the organizational sacrificial element identified. They viewed undergoing some of the training activities, especially those forced on them, as a sort of sacrificial opportunity cost for them. Rather than engaging in more beneficial activities in their opinion, such as developing their professional ambitions, they are forced to sacrifice this desire to please management's directives. Agreeing to participate in such organizational training activities, they emphasised, is often the only option for them, as if they show obstinacy, this could affect their jobs within their small business company. Thus, they often have to adhere to these stipulated conditions until they find alternative workplaces.

Relating this to the CRF of this thesis, it can be juxtaposed to the employer situational-context markers of ethical leadership, vertical communication and organizational culture elements of the framework. The main study employees 
narrated that their perceptions of employers have significant influence on how their sense of belongness (OI statuses) develops; wherein, when these are positively oriented it often results in EOI, otherwise a negative correlation would mean employees exhibiting EOD status.

\subsubsection{Receptive Attitude and Personal and Professional Fulfilment}

The final key element emphasised is the interplay between how receptive the employees are to undertaking organizational initiated trainings and how far these training activities go in helping to fulfil their personal and professional aspirations. These elements have root in the sacrificial feeling discussed in the preceding subsection. Employees in the pilot studies identified that often their mind set on the training they will undergo is pre-determined by whether or not it contributes to their personal fulfilment, thus determining the receptivity during the actual training. That is, if they feel the training will not contribute meaningfully to their professional career, they undergo the activity with a closed mind-set and vice-versa.

Comparing this to elements in the research CRF suggests a close alignment to the lived experiences narrated by the main study respondents in relation to employee OI situational-gap markers of personalised benefits including factors such as long-term career aspirations and sense of in-company worth.

\subsection{Respondents in the Main Research Study}

Table 2, as illustrated in section 3.6.2.2 (p.117) presented a summarised demographic overview of the research respondents interviewed. Their names were falsified to comply with confidentiality and disclosure dictates, in particular, as stipulated by the University of Gloucestershire code of research ethics.

The sectorial distribution of the interview respondents covers different sectors of the UK economy including Retail, Health, IT and Finance. Care was especially taken to 
make the sample distribution as diverse as possible in order to maximise the generalisability of the data collected, given the qualitative nature of the research study.

In terms of the gender distribution, the ratio of male to female respondents was 1 to 2 , with five male respondents and ten female respondents in total. This ratio highlights the important point that in this study women were more willing and open to being interview respondents than the men. A second inference from the data gathering process, though without sufficient statistical backing, is the deduction that certain sectorial occupations tended to have either a higher percentage of female workers while others had more male workers. For example, in the Health sector, the majority of the employees were females, ranging from GPs to support workers, whereas the IT sector was a more male dominated sector.

The ages of the respondents ranged from 19 years to 60 years. About $33.3 \%$ were in their 20 s, approximately $26.7 \%$ in their 30 s, $20 \%$ in their 40 s, and the remaining $20 \%$ were split between the under 20 s and over 59 s.

All the respondents have spent more than six months in their current respective SME organizations and table 3 shows the 'length in current SME' approximated to the nearest 'whole year' figure. This helps to substantiate the level of experience among the respondents since all of them have passed the often-mandatory probationary period of employment at their places of work and are fully fledged members of their respective organizations.

Also, a third of the sample (5 respondents in total) are employed in high-skilled roles, while the remaining two-thirds work in relatively low-skilled job roles. Among the five in highly skilled jobs, four are female and work within the financial and Health and 
Social care industries, while the only male works in the IT sector. The distribution of job skills level suggested some notable implications for the overall data analysis which are discussed further in section 4.6 (p. 194).

\subsection{Descriptive Overview of Respondents' Stories and Narratives}

The thematic analysis using the RDF starts with an initial summarisation of themes noticed from the transcribed interviewee narratives.

Figure 8 below shows a summary of the initial research findings, from which the data discussion and analysis emanates. Each external circle depicts the summarised highlights of findings for one of the SRQs, while the centralised circle describes the focal construct of this doctoral thesis.

Each of the SRQ themes was then further analysed by means of 25 semi-structured prompt questions that were posed to the interviewees during the individual interviews. These questions, Q1 to Q25, are detailed in the interview questionnaire template included in appendix I.

Each SRQ related data set is then cross-analysed across the fifteen research participant references to get an in-depth understanding and interpretation of the employee Ol focus of the SRQ questions and, where considered relevant, direct textural quotations from participants are included in the analysis that follows. Finally, each CRF component is analysed and interpreted in relation to the three main RQs. 


\subsubsection{Research Questions}

This study explored the concept of OI in SMEs by identifying its underlying determinants and specifically explored how training interventions as a strategic HRD influence individual employees OI process in SMEs. To address this line of inquiry, the main research objective proposed was to 'explore the use of training interventions as an employee organizational identification maker in SMEs'. From this main objective, the three following RQs were formulated:

RQ1: What does employee organizational identification mean within SMEs?

RQ2: What are the underlying markers of employee organizational identification in SMEs?

RQ3: How do training interventions influence the employee organizational identification process in SMEs?

\subsubsection{Sub-Research Questions}

To address the highlighted RQs listed above, the data gathering themes were further divided into seven sub-research questions (SRQs) and these are detailed below:

SRQ1: What is the perceived understanding of the OI within employees of SMEs?

SRQ2: How is OI characterised by employees of SMEs?

SRQ3: What are the perceived markers of employees' OI in SMEs?

SRQ4: Is training perceived as an employee OI marker within SMEs?

SRQ5: If Training is an OI marker, what degree of influence does it wield on employee Ol process?

SRQ6: How is training's degree of influence as an OI marker characterised by SME employees?

SRQ7: Can the employee OI process be influenced by customising training interventions within the organization? 


\section{Peception of EO}

-Routines

-Work environ

-Employee-Manager

Relationship(Leadership?

-Clarity of organisational purpose/End product Knowledge

- Open communication

- Leadership by example

-Employee(stakeholder) involvement in organisational policy fomulation and general processes

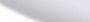

ס
Characterising Training's Influence on EOI process

-Positive: Creates assertive persons; often good for business; Physical working environ/Relationship; Better fit to

company needs

-Negative: Training means overqualified staff constantly leave 


\subsection{Data Analysis and Discussion}

Qualitative data analysis is primarily based on the researchers' interpretation of the data collected as opposed to the presentation of factual data in quantitative analysis. As asserted by Howitt (2010, p.104), "in qualitative research, it is not possible to make the rigid distinction between the results of the data analysis and the discussion of the data analysis that applies to quantitative research".

The data collection process has been previously discussed in detail in the previous methodology chapter of this thesis which primarily encompassed the development of the seven-thematic segmentation of the data collection process and the eventual findings analysis which was structured and designed to address the main research objectives and questions of this research. Following the IPA method, the interview textual data collected is thematically analysed and discussed concurrently within the relevant $R Q, S R Q s$ and $C R F_{x}$ addressed by the textual data analysed.

\subsubsection{Analysis and Discussion on RQ1 and $\mathrm{CRF}_{1}$ Outcome(s)}

The first research question (RQ1), set out in chapter 1 of this thesis and structurally depicted in the CRF and RDF shown in figures 5 and 6 respectively (pp. 84 \& 106) is restated below:

What does employee organizational identification mean within SMEs?

In addressing RQ1, a more in-depth layer of analysis is conducted based on the first overview of research findings as presented thematically in figure 8 in the previous sub-section. This stage of analysis identified five main components that characterise how SME employees perceive OI as a concept. These are represented by the below terms:

- Willingness to contribute more to the organization

- Identity within the firm

- Career aspiration alignment with company objectives

- Company investment in employee

- Tangible benefits from company 


\subsubsection{Willingness to Contribute More to the Organization}

A total of eleven participants from the interview sample inferred that $\mathrm{OI}$ to them meant their willingness to give more of their time, expertise and emotional support to their organizations without necessarily expecting anything in return. For example, Tanya, in response to Q2, said:

"I would say what influences my satisfaction for me is, are the people I work with are nice? If I'm happy to go to work in the morning or waking up in the morning and say oh...for me basically l'll say that kind of relationship basically makes you look forward, just makes you want to put more into it, to do more for the organization".

This resonates with Tompkins' (2005) and Boros' (2008) opinion that OI is an important research study field due to study results evidencing that identification with an organization often means commitment to the organization as well as having a sense of belonging which inherently conceives and creates willingness attitudes within such employees. That is, individual persons, who in this case are employees of SMEs, exhibit a certain belongingness personality within their immediate social environment (Stinglhamber et al.,2015; Ashforth \& Mael, 1989).

\subsubsection{Identity within the firm}

All the respondents interviewed shared this common perspective as a major determinant of their OI with their respective companies. From in-depth analysis of the interview responses, participants categorised this identity into the following two types:

- Self-Identity within the firm

- Group Identification within the firm

In explaining the role of self-identity within the firm, the consensus among the respondents is that this is defined by the level of importance or self-worth they feel they have attained in the organization. This may be in terms of their level of responsibility within the company and holistically the power they wield within their 
respective companies. Meanwhile, group identification within the firm was expressed as relating more to the closeness of the relationships formed with other members of the company at all levels, i.e. these could be relationships with colleagues, subordinates as well as their managers within the company. In one related instance, Tracy highlighted that:

"I am quite important within my organization, especially since another of my colleagues that has the qualifications I have left the firm late last year. Before she left we were the only ones that could officially assess and sign some official documentation for clients due to our professional qualifications and since she had been with the company longer than I had, she was mostly the go to person. However, since she's left everything now comes to me, as we are yet to recruit for her vacant position... The work is somewhat a bit tedious now, being the only one now basically doing the job of two, however I do enjoy it as I feel more confidence about myself and my importance and worth in the company seem to have leaped in bounds since then...obviously other colleagues need to consult me to approve such related documentation before giving it out to clients so they have no choice but to play nice I guess, and yes, I'm happy".

In the case of Kyle, he enjoys a healthy and fun relationship with his work colleagues as well as managers. He feels especially since he's one of the youngest in his firm, the managers sort of like him and are often very nice to him. Also, given the nature of the company's business, i.e. a restaurant, other work colleagues are often easy going and friendly, trading easy banter with each other. Another factor he noted is that because they work very long shifts together, more often than not they tend to make the atmosphere as lively and interesting as possible. His narration points to Kyle being increasing defined by his group relationships as he subconsciously refers to himself in a collective manner. Speaking below: 
"Oh, I always look forward to going into work, not just the work itself, as its quite physically demanding what we need to do, working in the front-end of the restaurant business, we work very long shifts. But my work colleagues are just fabulous, the vibes are just great! Even when my mood is not too cheerful, someone will say something that you can't help but laugh and comment on. My manager is also a nice fella, I sort of think he sees me as his somewhat younger bro I think... as I am one of the youngest staff at work, since I started work there, which is about a year ago now, l've never had any complaint about him, even when I call in late or sick off he never gives a fuss. And I sort of respect him for that".

These notions reflect some of the employee situational-gap markers embedded within the initial conceptual framework, namely questions about self- identity within institutions and group interactions, i.e. team relationships. This will subsequently be discussed in further detail in the next main sub-section addressing RQ2 and $\mathrm{CRF}_{2}$.

Cross-referencing with previous literature sources reaffirms the Stinglhamber et al. (2015) and Ashforth \& Mael (1989) opinions that OI is a derivative of the social identity perspective where individuals classify themselves and others into social categories exhibiting a defined personality within their immediate environment. That is, $\mathrm{Ol}$ is a function of self-identity and group interrelationships. Also, in terms of affective and evaluative components, Kyle seemed to be gradually developing some emotional feelings with his manager by asserting the thought that his manager sees him as a younger brother. Social identity theory affirms that this is feasibly possible, as these cognitive components could be expressed in the form of emotional attachment, feelings of pride and other positive emotions that are derived from organizational membership, i.e. Identification is a perceptual-cognitive concept which is a section of one of the four main tenets of the Ashforth and Mael (1989) notion of OI. This emotional feeling, as illustrated, is one of the gap bridges in Brenda Dervin's sense-making model. 
There is also an element of perceived power of the individual evident in the firm, as stated by Tracy. Power structure is a prominent component of Dervin's sense making theory from which elements of the conceptual framework were generated. Tracy appears to be hinting about certain power politics going on in her organization which can invariably be argued as part of the organizational culture of her firm. This could be argued as a tool for identity regulation within the firm in terms of communicating values and goals by creating a vacuum and then a perceived need for employees to identify more with the organization through sense-breaking and dream-building (Pratt, 2000). This, however, is not explored in further detail, as no other research respondents identified this as an Ol contributory factor per se.

\subsubsection{Career Aspiration Alignment with Company Objectives}

The third component characterising SME employees' understanding of OI is related to their perception on how their own career aspirations align with the company's objectives.

Ashforth and Mael (1989) as well as Levinson (1970) pointed out the fact that career goals form one of the drivers of commitment linked to $\mathrm{Ol}$ and this figured significantly in Ashforth and Mael's four basic assumptions in their 1989 literature on OI. Explaining this they said: '...Commitment might arise because that particular organization is a vehicle for one's own career goals. This leads to the fact that leaving that organization for another one, where these goals can better be fulfilled, is a possibility at all times...'

It is noteworthy to mention here that the interview respondents consider their own career aspirations of primary importance in comparison to their respective companies' organizational goals or objectives. On analysing the respondents' narratives, while employees suggesting huge disparity between their own career aspirations and company objectives demonstrated lower levels of Ol, those exhibiting close similarities between their own career ambition and their organization's 
objectives generally demonstrated better enthusiasm and EOI towards their employer.

Relating this to Gratton et al.'s (1999) explanation of strategic positioning as a function of fit levels varying from weak to strong, which also indicates where competitive organizational advantage can be gained, Gratton et al. (1999) explained that where a weak link is predominant, employees will implicitly not identify with the business and vice versa.

Thus, career aspiration is indicated as one of the indicators of fit levels within SMEs, i.e. the more aligned the individual employee's career aspiration is with the company goals and objective, the higher the OI level of the specified employee.

Explaining this, Rosemary highlighted that she views her current job within her firm as mainly for sustaining her and getting her bills paid and possibly developing more confidence in herself and saving up to go to University. She highlighted that it is mainly a stepping stone for her to transition according to her passion and career aspirations and she does not see herself spending her whole life working where she currently works or in the role she currently performs within the company. She commented:

"where I'm working now, it's only to pay the bills unfortunately... they are flexible in helping you achieve that, however l'm not looking to pursue my career within. It's just helping me to build my aspirations. So, saving enough money to do an online course or just helping me, steering to be able to pay all my bills, so that I can go to Uni".

This viewpoint seems also to radiate through in terms of Rosemary's level of satisfaction with her job, which indicated an average OI level. She emphasized this as follows: 
"I would say my level of satisfaction is 6 , If I could rate it to 10 yeah... It's because it doesn't stretch me... it's not challenging enough...Its not being. I'm not doing something new, it's the same thing every day. So that's why".

On the other hand, Debra, along with a seemingly higher Ol level, had a correspondingly higher work satisfaction level. She mentioned that her current profession has always been what she dreamed of as a child and practicing as a professional in that field now is just very fulfilling and motivating on its own and that makes her very happy at work and correspondingly identify with her company. Speaking about this Debra said:

"For me I would say my level of satisfaction is 8 mostly and firstly my ambition since I was a child was to be in this profession and it is a reality today which just makes me satisfied... It's an awesome feeling and one every day l'm grateful for because I know very well that not everyone has that privilege to do what they really want to do, especially in this country where things are very expensive and there's always bills and more bills to be paid, so some cannot even afford not to be working 2 to 3 jobs every day all week... Being able to practice is very motivating for me aside from other things...".

The level of career alignment to organizational objectives emerged as a main indicator of the OI levels exhibited by the interview respondents.

Further exploration of the perceptions interpreted from the respondent's narratives suggests a possible correlation between experiences and feelings on the respondents' OI statuses and their skill levels within the SMEs in which they were employed at the time of their respective interviews. While job skill was not explored in detail as a factor within the interview research questions, interpretation of the skilled 
workers' inferred Ol statuses does indicate this high-skilled category of employees had higher EOI status compared to their lower skilled counterparts. This is somewhat evident in the previous quote from the paragraph above from Debra. Tracy, who was working as a qualified account clerk, also somewhat echoed Debra's sentiments as to her satisfaction and belongingness with her company, thereby inferring a fairly high EOI status. However, Tracy did mention some more specific reasons why she feels this is so, especially referring to trust, respect for the owner-manager who acts as a parent-figure within the SME where she is employed, as discussed in section 4.4.2.1.2 relating to Ethical Management and Leadership Practices (pp. 168 to 175).

It would be worthwhile to explore this apparent relationship in future research work as an offshoot of my doctoral thesis.

\subsubsection{Company Investment in Employees}

Another way in which research respondents characterised their understanding of $\mathrm{Ol}$ was according to the degree to which their SME company invests or is ready to invest in their professional and personal development. This refers to the activities the company currently organises for employees to develop their skills such as leadership, job expertise, as well as provision of information on new industry developments. Often these activities are provided through various training related interventions, as well as other opportunities the company makes available to employees to facilitate their development. This stance does give an inkling of the overall research result in addressing the primary research aim to explore the use of training interventions as an employee organizational identification maker in SMEs'. However, this will be discussed in more detail within the subsequent sub-sections on $\mathrm{RQ3}$ and $\mathrm{CRF}_{3}$ (section 4.4.3, pp. 190 to 194).

Training as a construct has been defined as "... a planned effort by a company to facilitate employees' learning of job-related competencies" (Noe, 1999) and by Roy \& Raymond (2008, p. 49) as "a medium for developing human resources in business 
establishments, to motivate employees and improve their on-the-job performance". This translates that training is a key HRD tool that can be used by companies, including SMEs, to gain organizational competitive advantage as well as channel better employee performances across the organizations when the knowledge so gained is applied to their daily job routines (Appelbaum et al., 2000; Pfeffer,1994; Schuler \& MacMillan, 1984; Way, 2002).

All the respondents identified this factor in varying degrees within their narrative. Tanya, for example, explained that one of the main reasons she likes her company is because they invest in their employees to create a well-balanced staff force, who in turn willingly give their best to the organization in terms of performance.

Thus, as narrated by interview respondents, their employers provide relevant and appropriate channels for training activities to be used by employees for developmental purposes as well as to encourage better company performance. In instances where in-house organised trainings are not provided, funding is often provided for external courses or time off in-lieu is granted to the employee. The quotes below detail some respondents' narration of their organizational investment experiences and their contribution to their overall development.

\section{Rena:}

"... Due to the sensitivity of my profession as a dietician, I constantly undergo training which my company sponsors. They are mostly professional update and continuous professional development (CPD) related trainings...so yes, I'll say the company tries in that regard to be fair as they spend a lot of money. It may however also be attributed to government regulatory laws and guidelines which make it often mandatory to take some associated courses as a practitioner...". 
Wendy:

"My company only really train staff members when they are newly joined, during the induction periods, however, there is a mentorship scheme in place for those interested to sign up to for help with own in-company development growth path...."

Rose:

"We do invest in employees by training staff members occasionally, especially as the industry I work within (Health care sector) is heavily regulated, so diverse ever-changing governmental regulations are followed and this often warrants training sessions to be organised for staff members constantly. Also, when we have agency staff in, as a senior member of staff, I also train and show these agency workers across the home's facilities... That's about all the trainings we do".

Gilbert:

“...I work in the IT industry and as you can imagine, things are really very much fast paced. We constantly learn new things all the time. To cope with this, my company have an academy-like structure in place to offer short courses that will be beneficial to the business...l personally have, however, had to take some external courses which my organization's training academy did not offer, So, though the company did not pay for this training they willingly offered me holidays off work in lieu of time spent doing this, which of course I think is very brilliant and good investment which has good potentials in terms of return on investment".

Finally, Tanya:

“...l will chip in the training bit as well, I'll put it [training emphasized] as well, because that goes a long way. People might not look at it from... but looking at it from another perspective, if you are not trained to do what 
you're doing it could lead to frustration at work, it may lead to frustration at work, probably you're struggling with what you're doing, you don't have an idea of what to do or where to go and it's not every case you want to escalate. You're given your workload, you want to try and manage it as much as you can, rather than piling workload on somebody else...l kinda like got to where I am in the organization now through training. They actually invested in me, to bring me up to speed to what I know now... I said invest because, if I leave xx company today, I can actually manage a company myself, 'cause I know how the day to day running goes, I know practically how all sections of the company are run. They kinda like invested that in me, made me go through all the stages before the endproduct (that's me), so I have a knowledge of the whole thing entirely. So, they kinda like... I use the word invest because it's kinda like an investment, like when you put so much in someone, for that person to be able to stand alone... and do things.

For example, let me take, for instance, one of the old places where I used to work, we have different functions that we do to make up the whole department, they kinda invested in me to go through the whole like four units, I can do that because I've gone through all the units, I don't have to wait for one person to do one bit and then the other. I can actually process, do the whole process completely without escalating or without looking for someone else to help me.

So that's kind of like the investment l'm talking about, where you make the person total! That person can stand alone, that person can go through the day today with little or no assistance from colleagues or managers".

In concordance to the proportion of high-skilled employees among the respondents having higher Ol statuses as indicated in section 4.4.1.3 (pp. 154 
to 157) Debra, Tracy and Gilbert, a GP, account clerk and senior IT project analyst respectively, both lend some credibility to this assertion. However, so does Tanya who as an assistant store manager is not necessarily highly skilled but still exhibits a strong sense of belongingness and thus Ol status in her current SME organization, an outcome she mostly attributes to her immediate manager's caring nature towards her as well as satisfaction she derives from working with great colleagues in the workplace.

\subsubsection{Tangible Benefits Derived from the Company}

The fifth and final factor considered by the interview respondents as characterising their Ol status is evidence of tangible benefits received from the company. That is, the physical dividends they can obtain from their SME organization. About one-third of the research participants identified remuneration packages as top of their list in determining their identification with their respective organizations and this significantly determined the level of satisfaction on the job.

For example, Gilbert noted that on a scale of 1 to 10 , with 10 being the highest, his level of satisfaction is 7 which is largely as a result of his pay compared to industry standards. But he mentioned this is compensated for by the level of recognition and trust he seems to be given on the job despite having worked for the company for only about 2 years. Highlighting this he stated:

"In terms of satisfaction on my job, l'll say I have a high level of satisfaction, not in the monetary aspect of it because in terms of satisfaction some people might look at so many things, in terms of am I getting enough? Or is what I'm getting, does it compare to what my mates are getting? not from the same organization but in the same industry, so in this concept what I'll say is that my level of satisfaction is really high from the fact that I'm not looking at the monetary aspect but in terms of the responsibilities that l've been given as a person, that is, leading a team. Those are the kind of things that give me the satisfaction, you know, giving 
you responsibility is a big thing...So as I said, the responsibility actually drives you because if you don't actually have a responsibility, it means you're just there... without the responsibility you might not get the level of satisfaction you want, because you might just be a bench warmer in the office and that's not really what you want to do, but like I said, if you have responsibilities I believe without looking at the monetary aspect, although some people, like I said, will attach it to monetary aspect but I believe your level of responsibility should be what gives you the level of satisfaction you want on the job...."

Rating his satisfaction level within the organization and in explanation of what he means by the monetary aspect (See Gilbert quote above), Gilbert replied:

"... with 10 being the highest, level of satisfaction I will say $8 \ldots$ but in the monetary aspects if I was to look at the level of satisfaction l'll say maybe 7 ...yes...there is no commission on projects executed but in totality, when you look at the monetary aspect, we'll be talking about your salary, what are the other benefits attached to it. We do have some organizations, they don't have some schemes in place like life insurance, which they have in my company, so those are the kind of things I mean by monetary aspect, the bonus as well and the annual increment, so those are the kind of things that I mean by the monetary aspect I'm talking about...".

Comparing this to Ol literature, Riketta (2005) stresses the correlation between perceived $\mathrm{Ol}$ and Job satisfaction that summarily suggests a positive relationship between the two factors; as the job satisfaction levels increases, so also does the employee's Ol level, thus resonating with the findings from the majority of the research respondents and as emphasized by Gilbert's narrative. 
Conclusively, in response to the study RQ1, an in-depth interpretation of the research respondents' narrative within SRQ1 and SRQ2 suggests that all respondents have a positive employee organizational identification (EOI) as indicated in the $\mathrm{CRF}_{1}$ section of the conceptual research framework as opposed to employee organizational disidentification (EOD). Thus, while confirming the evidence of $\mathrm{Ol}$ as a construct, the research outcome suggests there were no ambivalent (neutral) Ol levels among the respondents as proposed by Kreiner and Ashforth (2004).

Ambivalent identification was defined earlier in chapter two of the thesis (Refer to section 2.5.1, pp. 30 to 33), as a dual state containing both identification and disidentification to an organization (Kreiner \& Ashforth, 2004). The absence of this finding in the current research may be arguably explained by the respondents' expression in their interviews of no out-rightly negative views of the company, which is an essential prerequisite for an employee to develop ambivalent identification within an organization ((Kreiner \& Ashforth, 2004; Elsbach \& Bhattacharya, 2001).

\subsubsection{Analysis and Discussion on $\mathrm{RQ2}$ and $\mathrm{CRF}_{2}$ Outcome(s)}

Following on from the discussion in the previous subsection, attention now turns to analyses and discussion of findings on $R Q 2$, relating this back to the $\mathrm{CRF}_{2}$ component of the CRF as depicted in figure 5 (section 2.12, p. 84)

The second research question (RQ2), set out in the introductory chapter of this research is restated below:

What are the underlying markers of employee organizational identification in SMEs?

To address RQ2, a further in-depth analysis of the initial interview findings produced eight (8) main component markers of employees' Ol status among interview respondents as listed below:

i. Vertical Communication and Clarity of Purpose 

ii. Ethical Management and Leadership Practices
iii. Company Image/ Identity and Culture
iv. Company Productivity
v. Work Relationships
vi. Personalised Benefits
vii. Perceived In-company Worth
viii. Training Interventions

These highlighted markers, presented in table 3 below, are all analysed and discussed as functions of the main OI Marker category, with the exception of training interventions which seems to play a dual role across both categories of OI markers. Each OI Marker highlighted either falls within the 'employer situational-context OI markers', or 'employee situational-gap OI markers'. An understanding of each term is presented in this section, highlighting its relevance to the employee Ol process. Based on the initial CRF, Table 3 below shows a comparison of the OI markers post interview analysis and initial markers included in the contextual research framework $\left(\mathrm{CRF}_{2}\right)$ components:

\section{Table 3 - Comparison of highlighted employee OI markers in SMEs, pre- and post- research interview sessions}

\begin{tabular}{|c|c|c|c|}
\hline \multicolumn{2}{|c|}{ Employer Situational-Context OI Markers } & \multicolumn{2}{|c|}{ Employee Situational-Gap OI Markers } \\
\hline Post-Interview & Pre-Interview & Post-Interview & Pre-Interview \\
\hline & - & $\begin{array}{ll}\text { Facilitated } & \text { training } \\
\text { Interventions } & \end{array}$ & $\begin{array}{l}\text { Organizational } \\
\text { Interventions }\end{array}$ \\
\hline $\begin{array}{l}\text { Vertical } \\
\text { Communication and } \\
\text { Clarity of Purpose }\end{array}$ & $\begin{array}{ll}\text { Horizontal } & \text { and } \\
\text { Vertical } & \\
\text { Communication } & \end{array}$ & Work relationships & Group interactions \\
\hline $\begin{array}{l}\text { Management and } \\
\text { Leadership Practices }\end{array}$ & Motivation & Personalised benefits & - \\
\hline $\begin{array}{l}\text { Company image and } \\
\text { culture }\end{array}$ & $\begin{array}{l}\text { Organizational } \\
\text { Identity and Culture }\end{array}$ & $\begin{array}{l}\text { Perceived In-company } \\
\text { worth. }\end{array}$ & $\begin{array}{l}\text { Questions about } \\
\text { self-Identity within } \\
\text { institution. }\end{array}$ \\
\hline Company productivity & - & - & - \\
\hline- & Employee Unlearning & - & - \\
\hline
\end{tabular}




\subsubsection{Organizational Identification: Employer Situational-Context Markers}

This section details SME employee Ol status markers that the employees are reactive too and have no control over within a small business. Drawing on Dervin and Weick's sense-making theories, both highlight the significance of a situated context in influencing an individual's thought process (Snowden, 2008; Weick, 1988). That is, there is a context external to the individual, often not under the control of the individual's (in this case employees) thought process (Dervin, 1998), which causes them to identify with their organization through the process of OI. Five main factors were identified within this category of OI markers: Initiated training interventions; Vertical communication and Clarity of organizational purpose; Ethical management and leadership practices; Company image/identity and culture; and Company productivity.

These OI markers are presented and discussed below with the exclusion of initiated training interventions, which addresses RQ3 and thus is discussed in section 4.4.3.1 (pp. 190 to 192)

\subsection{Vertical Communication and Clarity of Purpose}

Communication is one of the major elements highlighted by the research interview respondents. Not just any form of communication, however, with all the fifteen respondents expressing the notion that when their organizations' purposes are communicated to them in a transparent and clear manner, it helps them develop their trust in the company and motivates them to want to do more in achieving the company's goals, which essentially represents the Ol process. Respondents identified the importance of clarity, even in their daily on-the-job routines, within the organization to give them a better and more articulate sense of direction within the organization.

Some participants expressed the opinion that from their own personal experiences and their colleagues', it is sometimes obvious that management have not communicated their intended goals and targets appropriately to employees and this 
drastically affects not only their satisfaction levels on the job but also their morale and productivity as well as performance. Thus, in response, some respondents identified that this would be one of the things they would wish to change if they were part of management. Other respondents did however express the opinion that the clarity they get from their organizations' management makes their work quite enjoyable and easier to carry out. Expressing her opinion on this point, Binta stated:

"Looking at what the organization is all about... in as much as I know where the background is and the profit of whatever we're making is going, if the customer walks in... the way you present just to make sure the customer spends that extra bit goes a long way, because you know whoever at the end is going to benefit from it, so I help out in ensuring that extra bit comes into the organization. So, l'd say because of the way the work environment is and what the organization is all about, I mean not to say you'll always meet the nicest customers but you want to put that extra bit... going out of your comfort zone a little bit to top up the earnings of the company".

This notion was corroborated by Wendy who identified that

"before you start with them (her company), you have a tour of the company... to know what goes on at the back door... so whatever arm of the business you are, you already have an idea of what the whole business is about, so that gives you, that at the back of your mind, so wherever you're working from... it gives you that sort of thing like, you know where the end-product is. It makes you go into it and you're being trained..."

Speaking of one instance Tanya stated:

"If given the opportunity to change anything as management within my organization, I wouldn't really change anything... except possibly 
communicating better to some of the staff, especially our drivers. Several incident reports from customers often complain about them, especially the manner in which they handle their jobs... not good, not good at all, it does give the company a bit of a not so welcomed image and we need to constantly try to correct this impression in store. Unfortunately, we then might have lost a return customer who didn't bother to report any incident back to the retail shops.

I know the job the drivers do is hard and obviously physically demanding, but I feel the majority of their behaviour comes from not really knowing or better still not understanding the company's background and purpose well. I said understand because I know the company does an induction when you start off with them. But it's just the one time and I feel as if these drivers do not really let this information about the company sink in during their induction... What I might suggest the company does is to do some refresher courses for the drivers every so often and even though I know it will cost more money, being not such a big company overall, but I like to think that it would be more beneficial for the company in the long run, even to grow".

Relating this notion of communication and clarity of purpose back to the conceptual framework $\left(\mathrm{CRF}_{2}\right)$ section, horizontal and vertical communication markers were highlighted. However, vertical communication was the key and paramount form of communication implied in the interview respondents' narratives and stories. Explaining vertical communication Bartels et al. (2009), said it represents how 'different kinds of...communication, such as information about the organization's strategy or objectives and current developments, help employees to determine their position in an organization". 
Vertical communication helps define an organization and convey what it stands for (Dutton et al., 1994). It is often work-related, travels top-down (comprising mostly of information on company strategy) and bottom-up (information sent to management from the work floor level) within the organization's hierarchy (Downs \& Adrian, 2004; Goldhaber, 1993). This is evident in the narratives of the respondents. They affirm the need for vertical communication to be a constant practice within their companies, with those respondents that acknowledged that their companies already practice this highlighting associated benefits of improved $\mathrm{Ol}$ with their companies. This is expressed in various ways such as better motivation improved trust indices and, where relevant, better customer satisfaction, as opposed to when vertical communication measures are low or even almost non-existent within the company.

\subsection{Ethical Management and Leadership Practices}

Responses from the research interviewees in terms of their opinion on management and leadership practices in their company were in profound agreement. All the research respondents identified with this component of the research questions in general. They highlighted these management attributes as a significant marker and determinant of their exhibited OI statuses within their small business companies.

The majority of the respondents spoke significantly about the fairly good relationships they have with their managers (often these managers are also owners of the company), as small businesses are often characterised by flatter hierarchical structures. These cordial relationships have helped develop some associated feelings of respect, trust, as well and parent/older-sibling figure relational feelings which according to social identity theory are cognitive components known as emotional feelings within the sense-making frames of the relevant respondents.

Defining perceived respect in a CSR and OI related study, Farooq, Rupp and Farooq (2017, p. 958) said it is '...what provides employees with a sense of pride in their organization, as well as a feeling that they are valued'. They went on to highlight that 
employees who feel respected by their companies have a sense of esteem. This is in agreement with De Cremer and Tyler's (2005) assertion that respect mirrors employees' beliefs of being valued members of the organization.

Highlighting this further, Tracy and Rena both argued that despite their fewer years' experience of practicing in the profession, they do feel especially welcomed by their company's management and team leadership and made comfortable. This has helped them develop a sense of respect for the companies; moreover, it is relevant that they were even hired initially to take up positions that more experienced staff members had held before them.

Trust, on the other hand, is said to have the ability to influence employees' attitude(s) within an organization by fostering the belief that the organization is capable of and intends to implement its set purposes (Campbell \& Im, 2014); thus, it has the ability to reduce individuals' uncertainty in relationships and thereby to facilitate better social exchanges among employees and hence better performance (Chen \& Klimoski,2003; Schaubroeck, Peng \& Hannah, 2013). Campbell and Im (2014) argued that the more employees view their organization and its processes as trustworthy, the more likely it is that a bond of identification will form'.

More specifically, cognition-based trust, which refers to confidence in another's competence, reliability, and dependability in the domains that are relevant to one's work relationship with him or her, and affect-based trust, referring to an emotional attachment to another person that mirrors the confidence that each person is concerned about the other's personal welfare (McAllister, 1995; Schaubroeck, Peng \& Hannah, 2013), are the main players in relation to this study.

For example, Tanya, in explaining her reasons for enjoying her job role, had her manager to thank most for this. She said her manager goes all the way to ensure 
she's alright and also always attends to her physiological needs by bringing in lunch for her every day. Tanya said:

"I would say the level of satisfaction has to do with... the people I work with, my relationship with my shop manager... I'm happy with what I'm doing and the people are nice too. I do look forward to going to work... basically my relationship with my manager. I would take, for instance, when we go on dinner, I myself I hardly take anything in for my dinner but my manager makes it like a point of duty to always bring something in for me... she never misses it so I mean that kind of relationship makes you look forward even makes you want to put into it...".

This sentiment was also shared by Debra and Patricia, both working in a similar professional environment, who asserted that in comparison to a lot of stories they've heard from their colleagues from other companies within the same industry, they feel they have good managers who are their mentors. Buttressing this further, they shared the opinion that they are never too intimidated or deterred from asking questions or seeking pastoral advice when they feel the need. According to Debra:

“...Personally, I really do like and respect my mentor at work who you could say is my line-manager, he is always ever so helpful and encouraging. Especially as a professional, I just concluded my traineeship and have only been working as a full practitioner for a little over a year now in my current practice. And I say this with experience because the nature of our profession's training dictates you are moved around different practices over a period of three years every six months. So, I'm not flattering when I say my current mentor and manager is very good and accommodating. I mean l've had some awful experiences with other mentors over the last couple of years that I don't even like remembering...but I came through. So essentially my point is he makes 
my practice more enjoyable and with his experience as a practitioner, which is over 25 years by the way, compared to mine which is barely a year, he is a source book for most questions I have. I find it comfortable to seek his advice not only on client consultations but even on administrative issues".

Echoing this Patricia shared her own opinion:

“... I just honestly like my current place of work. From the activities and other things, the company does for the employees, I think they are one of the best practices in this region. The other day, we had a practice sponsored dinner party and this is not for Christmas, it was just randomly. These little things, I think, make us as employees happy and willing to commit themselves more to work. And I think our current managing partner who also happens to be my mentor in the practice is at the heart of it all. My interactions with him over the last two years are always memorable and I mean that in a good way. He does my yearly internal appraisals as well and always has good things to say about me and good advice I suppose as well, when I need it. In fact, 'cause in my profession you often have an external appraiser as well, my mentor's appraisal of me is always glowing and he wades in should the external appraiser raise any issues. I just think he genuinely cares for my professional development and welfare".

The last element identified in terms of management and leadership practices within the organization is parent/ older-sibling figure mentality, which, compared to the elements of trust and respect, was mentioned in their narratives by fewer respondents. However, the respondents that did do so were very passionate and focused on it being a crucial factor influencing the way they perceive leadership within their SME organization and thereby influencing their OI process. 
The overall consensus was that the more they see their managers, who are sometimes owners of the small business, as a parent or older-sibling figure, the more they develop a sense of identification with the organization. For example, Tracy recounted that actually she owes her boss, who is also the owner of her firm, everything she is today, in terms of the work opportunity given to her when no one else would give her a chance despite having the right qualifications. She stresses that as a norm, her boss acts almost like a parent to her and everyone else in her firm and he's always looking out for her and cheerleads her into achieving things she would never do in her own right even though she could accomplish them. In her words:

"...well one thing that really stands out for me is the proprietor of the firm, he's quite family orientated. So, you coming in is not just business, he's always wanting to connect with each and every member of his staff, both professionally and as well as wanting to find out about what they go through like individually outside of work. Because sometimes you might come into work, you know, looking sad, nothing to do with work, but obviously that will affect your job, you know, your level of productivity on that day. So, I think one of the things that I like about working in the firm that I'm in at the moment is the fact that he takes his time to notice,..., it's very very important for me that he sees. Sometimes when people are going through some difficult times in their own life, nothing to do with work, and obviously you coming in to work upset will genuinely affect your productivity for the day and maybe you need time off, it might help you and like regain a little bit of your strength, and come back working twice as hard as the way you were before, yeah it all depends...".

Tracy attributes this behaviour possibly to her boss's years of managerial and leadership experience, in which case she supposes, intermittently, he would have taken time off to reflect on his leadership acumen and developed skills 
that encourage people to stay longer with his small organization even if they eventually leave to follow bigger aspirations. She said:

“...Well I will probably say standing from the proprietor's point of view, it will probably be for the fact that he's been in the business for over 39 years and he would have noticed, you know, that people come and go, I think on the payroll system I think I'm number 32, so I'm guessing over the years he's noticed people have been and left and obviously he'll have taken time out to reflect on why these things are happening. So, this will help to better restructure the organization ...".

From Kyle's perspective, however, he sees his manager's impression on him as being that of an older-sibling figure, a brother. They seem to have a fun and healthy relationship and often during his narrative he inferentially assesses himself as being somewhat treated with favouritism. This he attributed to possibly his younger age compared to the rest of his colleagues within the company. On these points Kyle said:

"Work is pretty interesting for me and obviously an exciting experience, mostly because I enjoy a healthy relationship with my colleagues...especially my boss, I just think he's awesome in the way he manages the team. He doesn't necessarily micro-manage us, he sort of gives everyone their space to do their thing and only steps in when it's obvious there's a need. He can also be seen on the restaurant front house when we have busy bookings during peak periods. He just chips in often, even though I don't think it's his job to do that really. But anyway, he does that often. To me specifically I just see him as kind of an older bro, you know what I mean, he's always there for me both at work and even 
outside work. I just think he sometimes does treat me quite more favourably...".

Kyle also stressed the extent of familial relationship between himself and his manager even outside of the confines of the workspace, which further substantiates the idea of a parent/older sibling relationship existing between the two. Kyle mentioned this as follows:

“... For example, he's invited me for several family dinners and I do get along well with his wife and his two kids, who by the way are very awesome and fun as well. For me it's a very nice experience and I just see him as a sort of older bro which except for some uncles, I do not have an older sibling that I could have that sort of relationship with you know, to be honest. And I can tell you it's an amazing feeling and I'm never sad to be going into work any day since I started...To me, when I think deeply, I sort of feel it may be because of my age, I'm the youngest at work currently and maybe he just wants to protect me or maybe not, also maybe he sees his younger self in me?...l've never really asked him why and probably not going to, not to jinx it...".

In another instance during his interview, Kyle mentioned that his manager very much sees him as a younger brother and he has never had a cause to complain about him since starting employment about a year ago in his workplace. He even emphasised this is also the case when he's had to call in late or sick to work.

The parent/older-sibling figure, in the context of business organizations, except in organizational cultural dimension studies that explore the significance of parent-like (patriarchy) authority in small family businesses as well as child and family studies (Prince, 2006; Sherman, 2005; Teel et al., 2016), is essentially an uncharted concept. Based on the interview respondent's narrative and analysis within the 
context of small businesses, the parent/older-sibling figure concept represents feelings of admiration and respect for another often-older individual, based on how we perceive they relate with us. This means that how we perceive an older individual as being a parent/older-sibling figure is based on how they interact with us, which in turn helps us admire and develop a sense of respect for them.

All these aforementioned elements: respect; trust and parent/older-sibling figure, are, according to the respondents, progressively associated to be developed by the employees when managers show apparent care for them. Thus, in the context of this research, evidence from respondents points to the fact that care-giving managerial and leadership practices by managers comprise a respect, trust and parent/oldersibling figure making machine within SME organizations and ultimately these elements help motivate the employees to be more enthusiastic and productive at work.

\subsection{Company Image or Identity and Culture}

The third Ol employer situational-context marker emphasised by the research respondents is focused on highlighting how the perceived image(s) of the company as well as the in-company culture influence their individual OI processes.

Drawing on previous academic literature (sections 2.1; 2.2, pp. 20 to $24 \&$ section 2.9.2 pp. 45 to 48 ) and perceptions of the respondents, the company image is synonymous to the corporate or organizational identity. Meanwhile, according to Abimbola and Vallaster's 2007 study on brand, an SME's organizational identity and reputation are distinct attributes of the organization which are essentially related to 'what an organization is' in terms of...the behaviour of employees and corporate communication towards internal and external stakeholders of the company. This view is opposed to the classical notion of organizational identity being represented by such as company logo, design style and colour scheme. 
This corporate identity often also defines the organizational culture of the company, especially in SMEs, where the owners or managers often imprint their personality and characteristics on the company, and this is in turn gives the company its distinctiveness (Olins in Abimbola \& Vallaster, 2007; Rode \& Vallaster, 2005; Wickham, 2001).

Defining organizational or corporate culture, Hankinson and Hankinson (1999, p.136) stated that it is "... a company's overall philosophy, a set of values and beliefs that shape the way people think and behave", while similarly Deshpande' and Webster, in de Chernatony and Cottam (2008, p. 15), defined culture as "the pattern of shared values and beliefs that help individuals understand organizational functioning and thus provide them norms for behaviour in the organization". Both of these definitions substantiate Schein's (1984) Iceberg illustration of culture as artefacts, values and basic assumptions... all of which point to organizational culture as defining the identity of the company, its brand, without which there is almost a non-existent chance of employees "living the brand" (Schultz, 2003). Thus, in the context of this research context, both corporate identity and culture are interdependent on each other.

Expressing their opinions on how they perceive their company's image or identity and linking this to its culture, all the interviewees' described their company in mostly positive tones. The interviews progressed further by asking the respondents to characterise their company's organizational identity. Several collections of words were used to characterise their company's identity, including the following: Competent; Efficient; Compassionate; Supportive; Customer-focused; Staff-focused; Hierarchical Structural Uniformity; Effective Performance Management; Builds Staff knowledge-Gap; Friendly; Warm; Welcoming; Open to ideas; Good; Inclusive; Conducive Environment and Trustworthy. 
Speaking on this point, Rosemary stated:

"For me what intrigues me about the company is in relation to the name itself which is geared towards caring and essentially that's what I try to represent everywhere I go or am sent by the company. What I love about the company is that they are quick to respond when a client needs a service asap and they always have someone they can send over... they are competent and efficient, what they say they'll do, they'll do.

Competency in this respect is they are competent at their trainings...they are competent at their trainings, that is all I can...yeah that is the only thing I can say and also pay me, they never delay. Even though they are a small company, there's never an issue with getting your payslips or getting your payment or mixed hours that you've haven't been paid the right hours, they always have the right hours that you've worked".

Corroborating this positive viewpoint Ben noted that:

"The way people perceive it, l'll say people are compassionate and how can I say it, more private time with your relatives... I perceive them as compassionate as well, I remember the first time I joined them, whao, l've never seen anyone so loving, caring and friendly, I think that's basically why I'm still with them because l've been in other companies where staff are so nasty but these ones, they are just so, when you're down everyone is down with you, you know when you're going through any trying period, everyone goes through it with you, people are there to listen, people are there to, you know, give a helping hand. I've gone through difficult times in my life, where they have just been $100 \%$ wonderful, so I really hold them high, with high esteem, so they are really wonderful, wonderful people". 
Tanya also noted that:

"I mean, every employer, you know can't definitely be hundred percent, they will have places where, they still lack. But when it comes to employee management, I'll say they are brilliant. They handle their employees brilliantly, even volunteers, they organise get-togethers for them you know, make sure that we have the awards thing that we do as well, we have loads of activities we put together... just to make people come together. So there's that kinda like the family tree thing... when you're part of the organization, you're kind of like in the family, so it's like a small family just like the family thing, like you have a family somewhere. So, I'll say characteristic like they're fun to be with, ...very very supportive".

Gilbert also shared this opinion by saying:

"In terms of the company identity, l'll be looking at reputation in this, I'll say they've got, their image and identity is really the one that is, I can say is, mostly acceptable within the industry, in terms of what they do. And then in the area of IT, if you look at them, they have other competitors, but I think they are actually placed second among the competitors, in terms of the industry speciality, they are actually placed second, which means the company is doing what they are actually meant to be doing....it means they've not derailed from their focus, from their line of business".

Despite the really positive points expressed in characterising their organizations' identities, some interview respondents did also express some low points. They highlighted in this respect that their companies are not perfect and identified factors such as not being challenging enough, loss of trustworthiness from customers, as well as perceived inept handling of disciplinary cases, which they would change if they were in management. All these feelings and perceptions could be said to be quite indicative of the Ol statuses of the employees relative to their respective SME organizations. 
For example, Rosemary, continuing in her discussion about her company's corporate identity, stated that:

"... when it comes to efficiency, as well they might give an employee's name that might not necessarily be the person that shows up at the client's place. I noted that clients do not really like it when they send someone who they have not been told would be coming and I can understand from the company's point of view that they just give a name, so they can assure that they have someone being sent over. But for them, they'll like to know exactly who they're going to send over, I think they just do that so they can assure the client they've got someone coming but they're not sure, because they need to ring around to find out who's free to attend to the relevant client... And from my previous conversation with clients, they never get told that another person would be coming and that's happened all the time to two different clients we have been to that have complained about it. That's the only thing I can say they do not manage well...in a way this loses trust for them, yeah, the company loses trust in regards to the employees they send, are they true? Are they even trained? It puts a lot of question marks for them... yeah l've experienced this almost all the time, but again I guess it's down to the nature of the job and industry itself..".

Wendy also expressed some doubts about the company, stating that despite loving her job, her current job description is not quite challenging enough for her, as she does the same thing day in day out and after a couple of months the work has become too routinely monotonous for her.

Collectively, from the narratives of the respondents, it is inferentially apt to say that company identity was expressed in terms of certain positive and negative dichotomous attributes and, as a function of the small business owner or manager's 
own identity or other employees' behaviour, can help shape the practiced organizational culture and vice-versa. As respondents strongly emphasised that these factors play interlinked roles in developing their OI processes within their respective SME organizations.

\subsection{Company Productivity}

Within the employer situational-context OI markers category, 'company productivity' is another final element highlighted by the research respondents as influencing their Ol processes. Productivity itself has been explained in terms of tangibility of benefits or successes of a business organization; that is, elements of a company's achievements that are measurable.

The responses from the research participants corroborate the understanding that $\mathrm{OI}$ is associated with a number of positive organizational outcomes, such as work attitudes and increased job performance (Scott, Corman \& Cheney, 1998; Ciftcioglu, 2010), which have equally positive implications for productivity, efficiency, effectiveness and ultimately profit within the relevant organization.

About $80 \%$ of the respondents believed that seeing positive changes in relation to their organizations' productivity based on training activities or other organizational motivation activities goes a long way in helping them to identify with their organizations; also, vice-versa is the case if no change or negative changes are observed. Explaining this, Tanya cited the productivity dividend experienced as a result of necessary management induced changes made in her company as a source of identification for her. In her narrative, she said:

“... I think probably if there's too much change, 'cause you know people, the way people handle change is quite...eh.., if the change is positive, if I can see the reason why this change is being brought in, why they are trying to change this kind of system to another system, then I'm okay with it, but when you can't actually place your hand on why they are making the 
change, then I struggle with things like that...then probably you can see the effect of these changes made reflecting on your sales or you can see the reflection on the number of footfalls coming into your shop...number of people, customers coming into the shop... if you can see that, then you embrace the change...

But if you do your changes and then the footfalls decrease, your sales there's no improvement, rather than going up, it's going down, then that way you have to like take a step back and say where have we gone wrong, then we can fix it. The change, if you can see it in the sales, l'm $100 \%$ for it but if not...Yes, l'll say yes for me... At the end of the day, it's the productivity that everybody is looking at. If you're not hitting your target(s). I mean it's good, when you're not hitting your targets, you want to make changes where necessary to be able to hit your targets. But then if you're making changes and you're still not hitting the targets, what then is the use of the change that you've made. So, for me, yes, the productivity has to be the basis for you making those changes...".

Rena and Frederick were also in support of measuring their Ol process relative to company productivity. Rena asserted that her company's productivity is a function of her dedication and contribution as well as her colleagues' to ensuring the company meets its set targets and goals every so often. Commenting, Rena said:

"...productivity in my line of work is heavily dependent on customer satisfaction which is a function of how we the employees treat and relate with the clients. And from a personal perspective, I do believe I always put in my best performance for every client that walks through my office door, and each time I get a referral or positive feedbacks from a current client, I'm encouraged to put in more efforts. This is particularly true for me because I'm ever conscious that the last individual to occupy the post I 
now hold in my organization had over three decades of practitioner experience more than me with only a couple of years. Hence, I feel motivated when I get positive feedbacks about my productivity at work and am always happy to even contribute more in the company...".

In his own understanding, Frederick mentioned that his belongness to the company is encouraged when he gets positive feedback from both company clients and management themselves. He mentions that both his colleagues and line managers have given him deserved accolades on several occasions and this in itself motivates him to want to do more for the business within his job role.

Speaking on this point, Frederick indicated that:

"...I for one am really a workaholic and on many occasions have been commended by my colleagues and even managers on what a good job l'm doing at work and within my company. This then often has a ripple effect on me because I feel happy and this sense of pride just encourages me to contribute and do more to the company..."

To conclude this sub-section, company productivity, according to the respondents, not only acts as a marker for the employees Ol statuses, but also as a motivator that helps them develop resilient work attitudes within their organization.

\subsubsection{Organizational Identification: Employee Situational-Gap Markers}

The second category of OI markers highlighted within the conceptual research framework is the 'employee situational-gap markers' and these situational-gaps, according to Weick and Dervin's sense-making model, detail the on-going innate thoughts of an individual that they in time come to acknowledge need attending to for them to be able to make a rationale opinion about any given concept in practice (Weick, 2001; Dervin, 1998). Echoing this idea, the research respondents identified 
four main elements that help to fill their identified 'gap': facilitated training interventions; work relationships; personal benefits; and perceived in-company worth.

These OI markers are presented and discussed below with the exclusion of facilitated training interventions, which addresses $R Q 3$ and thus is discussed in section 4.4.3.

\subsection{Work Relationships}

Relationships are the fundamental basis of the interaction's humans have every moment of their lives, whether from person to person or group to individuals. This importance is also replicated within business organizations, with relationships generally defining the depth of associations in the workplace as well as the degree of interaction that goes on between parties engaged in the relationships (Shephard, 2013; Cartwright, 2013; McBain \& Parkinson, 2017).

SMEs, in particular, are quite unique entities when studying relationships in business organizations, because they often have smaller numbers of employees and because of the level of specification of job roles in such small businesses, the rate of productivity and performance are often defined by the degree of relationship existing between employees, whether person-to-person (i.e. employee-to-employee and employee-to-manager) or person-to-group. Relationships have also been proven to be important to the survival and overall living standards of humans, as we are generally known to be social beings by nature.

Thus, putting that into context, the research respondents' opinion about work-related relationships primarily focuses on such relationships being essential to their having a sense of belonging with their organizations. That is, they view work-relationships as an environment that's either healthy or not and that plays an important role in determining how they feel or their place within their respective SME organizations.

Expatiating on this further, three main subsisting relationship types were identified that foster better Ol development: manager-employee relationship; employee- 
employee relationship; and customer-employee relationship. Regarding the manager-employee relationship, interviewees described this from the perspective of good leadership, often deriving from their line managers or owners of the businesses themselves, and this element is particularly interwoven with the employer situationalcontext marker 'management and leadership practices' explored in an earlier subsection. Respondents viewed their level of satisfaction in the organization as synonymous to the satisfaction they derive from their working environment which is a function of the health of the relationship with their respective manager. Relating to this point, Tanya iterated that:

"I would say the level of satisfaction has to do with the working environment, the people I work with, my relationship with my shop manager, my colleagues as well... basically, it's the work environment. I'm happy with what l'm doing and the people are nice too. I do look forward to going to work... basically my relationship with my manager. I would take, for instance, when we go on dinner, I myself I hardly take anything in for my dinner but my manager makes it like a point of duty to always bring something in for me... she never misses it so I mean that kind of relationship makes you look forward even makes you want to put into it.... The work environment itself is brilliant. You know people relate to you with respect which for me, respect is reciprocal... it's really brilliant, the work environment is brilliant".

Also, Mandy mentioned that:

"... the industry I work in is very sensitive as you know and honestly one of the things that makes it worth the while for me is my manager, she's very down to earth and very caring and understanding... I mean having worked in a similar company before starting at this one about a year ago, I wasn't really enjoying work at all. Anytime any sensitive case or issue happens, which is often the case anyway, it just felt like my manager then or should I say the management in general just threw us to the wolves. I mean I 
quite understand the industry is very much regulated and often scrutinised due to the nature of the business but not once, not twice, she just acted like there was no loyalty to any one of us, even those that had been working there for over 5 years...and this is often very demoralising. The least you should expect from someone you've been working with for some time is to defend them to some extent at the least. But, this never happened. Which is why I had to quickly find an alternative and find myself where I am today which on my radar has a very more conducive environment for staff members, so staff stay longer here, compared to the previous company where there's hardly any quarter you won't see about 1 to 2 new starters".

These narratives substantiate the ethical leadership (EL) notion explored in the section 2.9.5 of the literature review chapter (pp. 55 to 56). Brown et al. (2005 p.120) cited that the dimensions of moral person and moral manager are the governing bedrock for its application, describing EL as attributes that demonstrate 'normatively appropriate conduct through personal actions and interpersonal relationships'... (Brown et al., 2005, p. 120). Thus, it could be concluded that most of the respondents, like Tanya, have experienced EL in their respective SME organizations and this positively contributes to their OI process development within the company.

Employee-employee relationship, on the other hand, as described by the interview respondents depicts a peer-to-peer or even subordinate-to-self relationship within their companies. Peers here meaning employees on the same job-level or status as the respondents and subordinates are the employees on a lower level or job-status compared to the interviewees. This relationship type they characterised as having a fun, friendly and joyous mannered ambience which is often full of energy and even helps lift their spirit or mood when emotionally down. Binta described this below:

"...coming to work every day for me is sort of fun really, my colleagues are just the most interesting bunch ever. Especially when the shop is quiet or 
a couple of us are on break, there's always one interesting gist [work gossip] or the other to laugh about. That's not to say we don't do our jobs as we should but we take time out as well to have fun both in and out of work. I'll even say that our rapport helps with our sales as well. So, take for example if a customer walks into the store and is looking to buy an item but wants some more convincing information about the item, I can easily go and ask my relevant colleague for them if I believe they are more knowledgeable in that department than I am. Sometimes they even come to attend to the customer instead and that in itself convinces the customer to purchase the item. We all mostly just get along. In fact, I'll say it's sort of like an unwritten prerequisite, especially with the shop being not massively big like some other companies in the business. I'll say that's one of our selling points as a company, this sort of a united front we present as workers in the shop".

On the other hand, having an unappreciative relationship with work colleagues can often be demoralising and makes it a struggle to work every day. Rosemary mentioned this point:

“That's a good question, because I don't feel like many of my colleagues feel like I'm a relevant person within the company. I suppose the way I see it, I'm just someone who's bothered to show up when needed and that could as well be anybody really... It hadn't really bothered me to be honest like I mentioned earlier, this is not the job I see myself doing in the near future but still asking me now, I suppose I'm not too happy about some of their disposition towards me but hey...bills got to be paid for now till I sort myself out and go to Uni I suppose...". 
The third work-related relationship some research respondents referred to is the customer-employee relationship. Fewer respondents highlighted this but the $20 \%$ that did were quite emphatic about it. Rosemary said:

"...yeah, I definitely feel like I am a relevant person with the company. Mainly because I know the individuals I'm working with...they usually need a lot of support and someone that would believe in them and I think that's what my role... that's where I come in, 'cause I do enjoy motivating and encouraging people so yeah... knowing that I could encourage that individual or help them to build even more independence than they normally would...".

This relationship type the researcher interprets as some sort of 'reverse customer service' whereby, like in conventional customer service scenarios, the employee serves and attends to the needs of the customer or client. The reverse customer service however paints a reverse picture whereby the employee derives some sort of benefit from serving the customer or client in a specific capacity. This action, as affirmed by the respondents, tends to be quite motivational for employees.

\subsection{Personalised Benefits}

The next employee situational-gap marker influencing employee Ol Status is the personal benefits employees derive from working in the SME organization. The respondents identified this as alignment of organizational goals with their individualised life-long aspirations, professional aspirations and self-aspirations.

An average of ten small business employees, which is approximately $67 \%$ of the total research sample, expressed strong convictions asserting the opinion that what they think or feel they can get from their respective organizations is an apt indicator of how involved they are or will be within those organizations and definitely plays a salient role in influencing their Ol statuses, within their organizations. 
Affirming this, Tracy said:

“...For me I believe one factor that could make me perform better or act better within my company, is to soak up the opportunities available to me within the company, to help develop my skills and expertise, so I can achieve my life-long dream of doing accountancy. Ever since I was a little girl, l've always dreamed of being an accountant. But despite it being a long and bumpy journey, each day brings me closer to achieving this dream.

Thus, when at work I do not complain often and tend to take on all the job duties given to me always! I just see it mostly as a learning curve for me to gather as much experience as I can possibly gather to improve my expertise in my chosen profession."

Also, Frederick mentioned that:

“...I mean I do like my current organization and all but essentially l'm often motivated by being able to work to earn and pay for my necessary expenses and bills, and I just presume the harder I work, the more compensation and company benefits I would normally get. This just encourages me to work harder or smarter and reap as much benefit as possible. I have to say however that I do really like my company, though customer focused, they are somewhat employee oriented and do cater for our welfare... All these things they offer make me like them better and make me feel like l'm part of a good company".

Rosemary also stated that:

“...Honestly, while I like my job, especially the clients I help out with, I'm mostly there to help me pay my bills and save up to study at Uni.(I'm 188 
taking some online courses now to facilitate that decision). So yes the company offer various training and developmental packages to help employees' development and on-the-job skills. But if given a choice, honestly, l'd rather not undergo those trainings, especially the routine ones".

The presented idea of personalised benefits as described by employees' narratives above, plays a significant role in influencing their Ol status within their respective small business organizations, which aligns to Gratton et al.'s (1999) idea on the need for a strategic fit between an organization's goals and objectives and the employees' own individual aspirations. A strategic fit then, in turn, facilitates pronounced competitive advantage for the firm.

\subsection{Perceived In-company Worth}

Another employee situational-gap OI Marker as identified by research participants in small businesses is based on their view of their in-company worth or importance. Respondents viewed their responsibilities in-company, when perceived as integral to the day to day operational activities of the small businesses, as a measure of their worth within the business and contributing to their self-acclaimed importance. They considered this perceived self-importance, or worth within the firm, to be a motivational factor in determining the Ol statuses within the company.

Tanya mentioned that she does identify with her company because "...they do try and make you into a total person..." For example, she stated that she could now conveniently manage a shop on her own, (even set one up) if she were put in charge. This she affirmed to be a function of opportunities and training activities provided by management of her small business organization. Similarly, Ben also identified with this assertion, exploring it from the viewpoint of reciprocity of respect displayed among employees of the company, stating that: 
“...Everyone in my company is respected no matter the job role you are doing, or the daily duties assigned to you...there's just this sense of satisfaction displayed and you experience a feeling of being of some worth to the business..."

Other research respondents also affirmed this notion, explaining that their incompany responsibilities and how seemingly important these responsibilities are to the products and services provided to company customers determine the extent to which they feel a sense of belonging with their small business organizations. Expanding on this they noted that the more important their respective roles and responsibilities within the business are, the more they develop a sense of incompany importance or worth, consequently facilitating the development of EOI statuses within the business.

\subsubsection{Analysis and Discussion on RQ3 and $\mathrm{CRF}_{3}$ Outcomes}

$\mathrm{RQ3}$, the third research question addressed by this thesis, is as written below:

- How do training interventions influence the employee identification process in SMEs?

Following the previous analysis and discussion sub-sections on employer situationalcontext OI markers and employee situational-gap OI markers earlier in this chapter, this section focuses on exploring the concepts of 'initiated and facilitated training interventions' and how understanding these concepts addresses the third research question.

\subsubsection{Initiated Training Interventions}

All the research respondents identified training as a key determinant of their $\mathrm{Ol}$ statuses in their respective small business. They defined initiated training interventions as training activities or programmes that their small businesses plan, organise and implement. These could take various forms such as e-learning courses, 
internal or external trainer facilitated in-class trainings, workshops and even off-thejob training programmes. The contents of these training activities could be routine in nature or developmental as described by the study respondents.

The types of trainings categorised as routine often comprise mandatory trainings stipulated by governmental establishments relative to the industry in which the SME organization operates. These are often organised and implemented within such companies in compliance to industrial and governmental rules, a perception that mirrors some of the inference generated from the initial pilot study conducted as detailed in section 4.1 (pp. 136 to 145) and more specifically sub-section 4.1.2 detailing the sub-heading on force or coercion versus organizational control parameters (p. 143). For instance, as a learning disability assistant, Rosemary mentioned her employers routinely enrol her on online update courses to be completed remotely which include data protection when handling client data, manual handling practices, as well as use of equipment. This is also echoed by Ben whose work in a health care home means regular mandatory training is scheduled for him to ensure he is well equipped to deal with service users' needs, is kept informed of general industry practices, and is protected from legal implications of such ignorance.

For example, Ben mentioned:

"... Last year, we all had to sit in a video training to understand people with sight disabilities and how they see things, it was quite an eye opener for me, as I have never felt so much empathy for my service users before attending the training. The video was interesting as it visually depicted how people with such associated conditions see what people with no disability take for granted on a daily basis. These included crossing the road, reading, eating, it was just like a tunnel vision and it really changed my mentality henceforth in terms of showing empathy..."

However, nine of the fifteen study respondents in the main study ascertained that in addition to providing such mandatory training, their SMEs organise, tailor and 
implement training programmes and courses to meet the developmental needs of their employees.

For example, arguing for the above, Gilbert highlighted the opportunity he was given to disseminate some of the knowledge he acquired from an external training which his SME organization facilitated by giving him time off in lieu despite not being financially responsible for the associated cost. He mentioned that this opportunity was mutually beneficial to both himself and his company, as he was able to put into practice the knowledge he'd gained, hence improving his professional expertise further, while his company benefited from his being able to transfer this knowledge to other employees.

In his words Gilbert noted that:

“... I do think to be honest, some of the trainings I have undergone on my own are equally beneficial to my company and myself. For instance, one of the trainings I did externally some months ago, the training academy in my company did not offer that particular course, but I wanted to do it, 'cause I knew it is quite important certification to have as a project analyst. So when I spoke to my line manager about it, he said the company couldn't sponsor me but he will give me time off to attend the training.... when I got back I did disseminate the knowledge I acquired to the rest of my team, 'cause as a senior analyst I remotely manage a team of five junior analysts and that was satisfying being that it made our work as a team more productive as well as I was able to put into practice the knowledge I have gained, hence the mutual benefit to both myself and my company..."

\subsubsection{Facilitated Training Interventions}

Research respondents' defined facilitated training interventions as training activities they independently undergo to boost their expertise and skills development. Hence, their SMEs are not active initiators or participants in this form of training 
interventions. Despite their SMEs not playing an active role in this type of training, the research respondents emphasised that their organizations provide good support systems that enable them to engage productively in such training activities. For example, Gilbert stated that:

“...There are some trainings I have to pay for on my own as part of my CPD: Continuous Professional Development requirements, the company does not pay for it but gives me time-off to take such trainings, as they are often off-the job and they do this without affecting my normal annual holiday entitlements. I think this is really a great thing... another thing they do is giving me the opportunity to experiment with my newly gained knowledge in my job with the firm, often with positive outcomes. This then encourages them to recommend everyone with similar roles as myself, in the company to undertake the training by part-sponsoring the total cost of the training..."

Echoing the above sentiments, Tracy also mentioned that:

“...at one point, I was shying away from taking one of the required professional exams in my profession...the next time my manager, who is also the company owner, inquired about this and I told him I'm yet to take the exams, he said he was going to help me pay for it to motivate me and I should go ahead to schedule the exam for a near-future but suitable date... I mean I was grateful but also amazed at the same time, cos the exam fee was well over $£ 500$ pounds...so l'm just indebted to him in a lot of ways..."

Thus, partaking in such initiated or facilitated training interventions encourages and motivates the research respondents to feel a better sense of belonging with their respective firms and hence more positively skewed and manageable OI statuses within their respective SMEs. 
These outcomes substantiate the impact training as an HRD construct has in influencing employee $\mathrm{OI}$ in small businesses and in general adding nuance to the wider strategic HRM field of research in ways of using HRM/D tools to improve employees' sense of belongingness with an organization. Employee Ol has been proven to improve organizational productivity, especially in the case of small business employees who are integral to the productivity of their organizations (Riketta, 2005).

\subsection{Sectoral Differences in Employee Ol Outcomes}

Outcomes of data analysis of the textual data collated from respondents did not show notable differences in the Ol statuses of employees employed across the six various employment sectors in the UK (see table 2, p. 117). As various employees within different sectors indicated similar employee $\mathrm{Ol}$ statuses within their respective current place(s) of work.

\subsection{Job Skill Influence on OI Outcomes}

In contrast to unnoticeable differences in the sectoral differences in relation to the $\mathrm{OI}$ status indicated by the research respondents, differences in Ol statuses based on different skill levels of the interviewed respondents were noticed. This was however not probed further during the interview process and it would make for a future area of research for my doctoral study. However, based on the methodology of IPA I employed in this study, bounded by my own work experience, it is probable that these differences (Highly-skilled respondents having significantly more EOI status that than respondents in low-skilled job-roles despite some being in the same industry. For example, Health and Social Care industry) could be related to the incompany self-worth the employees feel. This is one of the parameters of employee situational-gap markers in the CRF. A further rationale for this may be because the respondents with highly skilled roles are more likely to be in high-demand in their places of work due to their level of expertise and they are also likely to be paid significantly higher when compared to the low-skilled employees 


\subsection{Summary}

Chapter four presented the analysed textual data set, collected from the research participants. It then presented analysis relative to the primary research questions. This was followed by in-depth discussion of each of the OI Markers presented, which were identified by the research respondents as influencing small business employees' Ol statuses within their organizations. The chapter concluded by considering the dual functional role of training interventions as both an employer situational-context OI Marker and an employee situational-gap OI Marker. The final chapter will expand further on the textual data set presented within this chapter and conclude the thesis by providing relevant policy and industry recommendations for small businesses within the UK. 


\section{CHAPTER 5}

\section{CONCLUSION AND RECOMMENDATIONS}

“...Wherever you go, there you are..."

(Kabat-Zinn, 2004)

\subsection{Introduction}

At the inception of this research study, I set off to explore an organizational behavioural subject with a central significance in organizations from my experiential point of view. The several turns and curves met on the research journey however helped refine and shape the completed study into what is documented within this thesis.

The particular organization behavioural attribute as contextually researched in this study is situated within an equally important context of small businesses and their employees who are vital organizational actors in my opinion, a view supported by many organizational behavioural extant literatures, as they are often organizational actors integral to the successes often evident in SMEs. Thus, this research ultimately proposes the mediating role that organizational interventions, with primary focus on training activities, can play in improving the presence and maintenance of this organizational behavioural attribute among employees of small businesses in the UK.

My final chapter begins with a brief description of key highlights of the four earlier chapters. The chapter narrative details the purpose and objectives of the research; the methodology and theoretical approaches adopted; as well as the key novel findings of my research from interviews conducted with the respondents. Later subsections discuss the implications for business organizational practices, contributions to the academic body of knowledge, and limitations encountered in carrying out the 
research. Finally, the researcher offers pointers to further areas of future research, based on the subject and context of her study.

\subsection{Purpose of the Study}

The primary purpose at the onset of this research was to understand how HRD practices, with an emphasis on organizational training interventions, can influence the employee organizational identification process in an SME context. To accomplish this, the thesis set out three specified research questions that reflect the primary aim and the understanding that training interventions may not necessarily be the only factor influencing employee $\mathrm{Ol}$ processes in business organizations as reflected in extant literature (Boros, 2008).

Thus, to have a well synthesised thesis, a conceptual framework was developed to capture the essence of the three research questions and underpinned by the sensemaking theories of Karl Weick and Brenda Dervin. One rationale for combining these perspectives of sense-making was that it would enable capture of the cognitive experiential knowledge of individuals from Weick's (2002) perspective and the use of this experiential knowledge for bridging cognitive sense-making gaps (Dervin et al. 1993; 2003), within the setting of a dynamic employer situational-context, with this experiential knowledge and context often essential for organizational studies.

Sense-making involves "structuring the unknown..." by "placing stimuli into some kind of framework..." that helps "to comprehend, understand, explain, attribute, extrapolate, and predict" in such a context (Waterman, 1990, p. 41; Starbuck \& Milliken, 1988, p. 51). It also “...serves as a springboard into action" (Weick, Sutcliffe, \& Obstfeld, 2005, p. 409). That is, an outcome of sense-making is to facilitate future activities within a given context. This especially holds true for most SMEs as they are often by nature target-driven and continuously growing to proactively reflect the dynamics in the relevant industry (Valadez, 2011). 
Relating this to the research context, the dynamic employer situational-context is the small businesses, while the cognitive experiential knowledge is derived from analysis of the respondents' oral interview accounts regarding their related Ol experiences within their respective SME companies. That is, every interview respondent's story was retrospective, representing cognitive experiential knowledge shared with the researcher in relation to their workplace.

Another rationale for the use of the sense-making theories to underpin the Ol study is the need to articulate the unknown. That is, the interview process itself actively provided the respondents with the avenue to understand what organizational identification means contextually to themselves. The interview respondents were able to gain this understanding from the explanations and stories they themselves narrated during the interview process, since this process provided an avenue to reflect on their experiences relative to the OI questions they were asked. Therefore, in addition to the researcher playing the role of making sense of respondents' stories, the interview respondents were themselves engaged in a sense-making adventure during the interview sessions. According to Ancona (2011, p. 4) "...sense-making involves - and indeed requires_an articulation of the unknown, because sometimes trying to explain the unknown is the only way to know how much you understand it". Thus, it was quite interesting to see from the reactions and insight provided by the respondents how the interview process was enabling them to think and make sense of what they themselves do and how they react in relation to "belongingness with" their respective small business organizations.

\subsection{The Primary Research Findings}

The findings of the research relative to the respondents' stories told and the thematic analysis of these stories as detailed in the previous chapter are briefly articulated in this sub-section. The primary research outcomes relative to the main research aim, objective and questions are itemised as follows: 
i. SME employees characterised their understanding of $\mathrm{Ol}$ as a function of five main components: willingness to contribute more to the organization; Identity within the firm; Career aspiration alignment with company objectives; Company investment in the employee; and tangible benefits from the company. The first reflects the employee's desire to give more to his or her company with or without expecting any reward back; identity within the firm relates to the perception of each individual's own image and self-worth within the company; the third emphasises the need for alignment between the employee's career goals/ambition and the set organizational goals and purpose; the fourth elucidates the significance SME employees attach to their organization's investment in their total person as an employee of the organization; and the final component is a measure of the tangible rewards employees get back from their employers.

ii. From post interview analysis, five main employer situational-context Ol markers were evident in the stories of the interviewed employees; each defining an intra-organizational attribute representative of factors that influence the organizational identification process of the employees, and over which individual employees do not have direct control. Rather, small business employees experience these factors as a function of individualised integration or interaction with other actors within their defined small business organizations, such as managers, colleagues and subordinates. These factors include training interventions organised or initiated by their company; vertical communication and clarity of organizational purpose; ethical management and leadership practices present within the organizations; the company's perceived image which is a function of its organizational culture; and the evident employee productivity within the company.

iii. The third key finding of the research is the employee situational-gap OI markers, post interview analysis. This category of OI markers details factors 
that individual SME employees have cognitive control over, such as how they perceive their work interrelationships; how esteemed their sense of incompany responsibility is or how they view their self-worth within the firm; benefits such as career aspirations associated with their function within the small business organization; and training interventions organised or facilitated by the organization.

iv. Training interventions did influence the employee Ol status with the organizations. Viewing these training interventions as being a function of 'initiation' and 'facilitation', these SME employees view initiation as meaning training activities that small businesses routinely organise for their employees and which have a wide range of uses, often for compliance purposes as well as on-the-job capability development. This aspect of the training interventions is categorised in the revised $\mathrm{CRF}$ as an Ol employer situational-context marker, as most employees suggested that they are often routinely required to complete these training activities and do not necessarily have a choice as to their participation.

On the other hand, the second aspect of training interventions that influence employee Ol within their small businesses relates to training activities employees participate in of their own volition. Born out of the desire to develop their careers and professional prowess, employees suggested that they participate in some training activities which are not necessarily organised by their employers or that are externally attended. Explaining further, however, the interview respondents asserted that their companies do support them by facilitating their participation in such forms of training. Their small businesses actively foster an organizational culture that embraces employee selfprofessional development through their facilitatory actions or behaviours. For example, some research respondents identified that they sometimes engage in training activities external to their firm which are beneficial to their overall 
career, and even though the company does not always provide financial support for such trainings, they often time give time off in lieu for attending such programmes.

v. Parent/older-sibling figure (POF) is noted to be a strong employee OI status indicative factor within small businesses. This POF factor emerged to be a primary function of intra-organizational management and leadership practices within the employer situational-context OI markers category of the conceptual framework. The POF is expressed in trait-like manner, often by the managers, who are sometimes also the owners of the businesses, demonstrating seemingly protective and defensive behaviours towards their employees. Moreover, closer analysis suggested that POF is secondarily a cross-role OI marker, as it also has close resonance with the work-relationship factor within the employee situational-gap markers OI category. With reference to this cross-role status of POF notion, it simultaneously serves as both a motivational factor and a group interaction facilitator for employees within SMEs.

vi. Another finding evident from the research is the duality of roles small business owners play within their companies. About $85 \%$ of the research participants identified their managers as also the owners of the small businesses for which they work. While this is not necessarily unexpected, the newness of this finding is the role these owner-managers play in significantly influencing their employees' OI with the business through demonstration of the POF traits, as well as their employees' intent to stay with the firm for a prolonged period of time.

vii. In the context of these research participants' narratives, $\mathrm{OI}$ is expressed as a vector function with both magnitude and direction. While the magnitude aspect of this function is not explored in detail in the research, it poses an area for 
future research. However, the directional aspect of the Ol concept suggests small business employees exhibit neither a neutral nor an ambivalent $\mathrm{OI}$ status. Rather, at each point in time they have a measurable sum of $\mathrm{Ol}$, which can either be considerably lower or higher in comparison to others within the company. Hence the suggestion that $\mathrm{OI}$ involves a vector function between points $A$ and $B$, with point $A$ being the lowest $O$ l level within a small business and point $B$ the highest attainable OI level. This is depicted by EOD and EOI status respectively within the revised conceptual framework (see Figure 9, p.203).

viii. Another crucial key finding of this study is the sectorial and skill level differential evident in the stories and narratives of the research respondents. As discussed in section 4.5 and 4.6 (p. 194)

\subsection{Revised Conceptual Research Framework}

An illustration of the revised conceptual research framework included in figure 9 below shows the two key categories of employees OI markers within a given small business organizational context based on interpretation of interview data collected from research respondents.

The diagram consists of components including the employer situational-context markers comprising: initiated training interventions, vertical communication and clarity of organizational purpose; ethical management and leadership practices; company image/identity; and culture. Meanwhile the company productivity and employee situational-gap markers comprises facilitated training interventions; work relationships; personalised benefits; and perceived in-company worth. All these components emerged as influences on small business employees' OI process and indicators of their OI levels. 


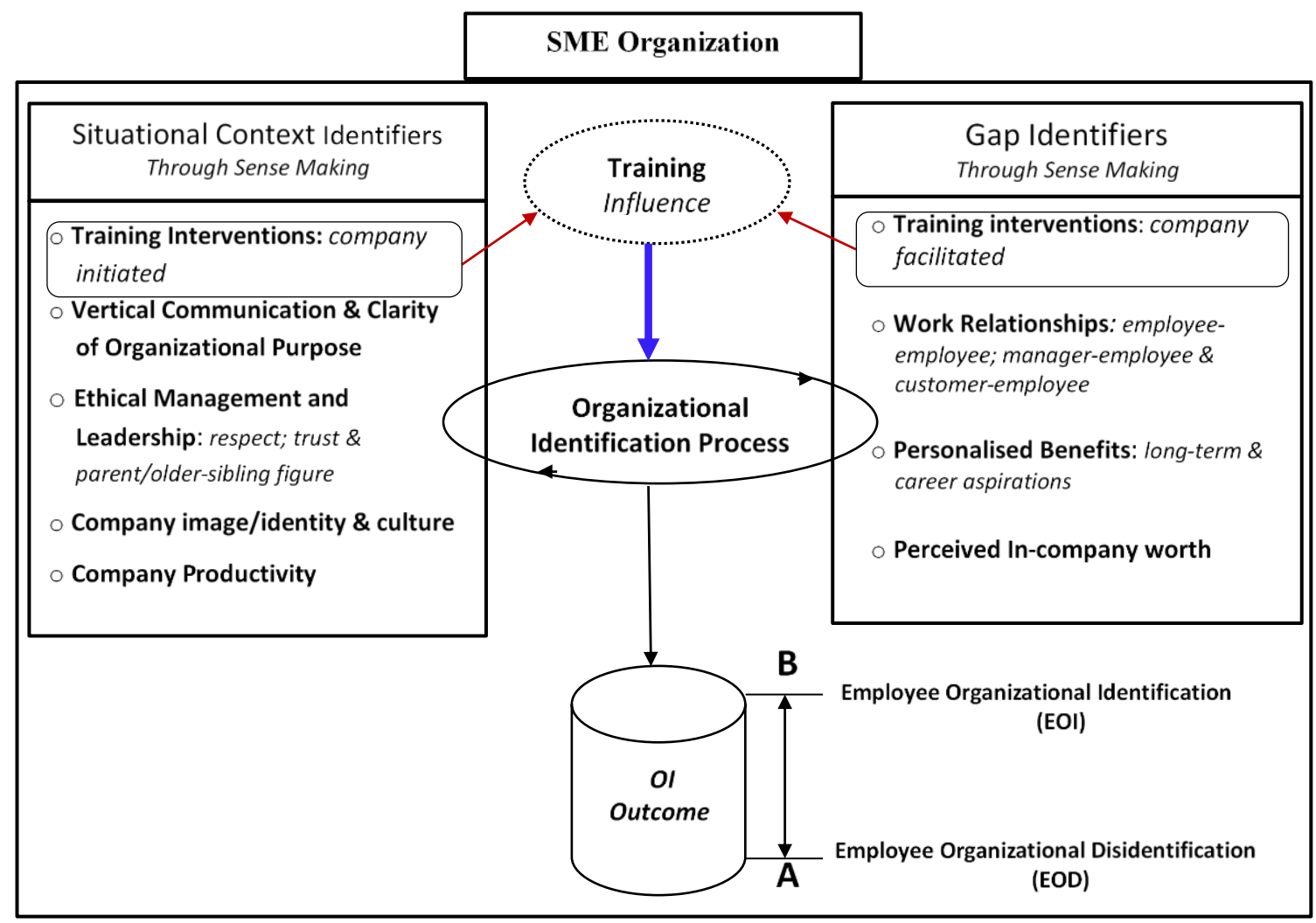

Figure 9: Revised Conceptual Organizational Identification Research Framework

From a comparative view the revised CRF differs from the initial CRF in the deemphasised relevance of some factors such as horizontal communication, employee unlearning and motivation, which led to these factors being excluded during the post-interview analysis of respondents' stories. Motivation could however be said to have been replaced by the elements of ethical management and leadership practices in small businesses which are often characterised by feelings and actions of respect and trust as well as experiencing a parent/older-sibling figure relationship, often between owner-managers and small business employees.

Also, there seemed to be a dual categorisation in terms of the role of training intervention as an OI marker in influencing SMEs employees' OI process. Rather than being exclusively within the employee situational-gap OI Marker category as shown in the initial CRF, the revised CRF shows that training intervention now also 
falls within the employer situational-context OI Marker category due to the companyinitiated aspect of training interventions.

\subsection{Organizational Identification Employer Situational-context Markers}

Employer situational-context markers, as illustrated in the revised CRF and relative to this research context, represent intra-organizational factors or attributes exhibited by actors within small businesses and experienced by employees within these businesses. A defining factor in relation to this organizational identification marker category is that the said employees do not have control over how these factors are displayed within the company and review of their narratives indicated that their perspective on this is only from an experiential point of view. That is, employees lack essential control to dictate how these factors occur in the company, and their only interaction with these factors is based on their experience of them and possibly how they react to these experiences.

That is, the perception of each of these factors is from a recipient-actor (employee) perspective rather than a giver-actor (owner-manager) perspective. These factors are shaped, to a large extent, outside them as individual actors. These factors are 'developed' by others, who, whilst cognisant of staff in the company, make decisions that are to a larger or lesser degree shaped by the decision maker's perspectives (and hence without considering staff views). Thus, effecting changes in these factors is beyond the control of employees and lies within the jurisdictive control of the owner-managers of the small businesses. The contextual explanation for each OI marker is briefly explained further in the next sub-sections.

\subsubsection{OI Marker: Initiated Training Interventions}

Inference from the stories and narratives of the research respondents suggested that training interventions initiated or proposed by management in small businesses are a predictor of employee Ol status within small businesses. The research respondents indicated that their companies do organise some training activities to improve their 
skills on the job. These could either be implemented in the company premises or external to the firm. Such training programmes consist of mandatory or compliance related training courses within the organization. These training interventions are also deployed on a regular routine basis and are termed initiated-training interventions in the context of this research. Together with the second category of training termed facilitated training interventions discussed later in section 5.5.1 discussion on employee situational-gap OI markers (pp. 210 to 211 ) such interventions have been arguably responsible for creating or investing in creating the total employee, according to the interview respondents, as small businesses tend to be quite supportive of their employees. This could also be argued to be a strategic HRM action of the organization in a bid to maintain good employee retention figures as well as a developmental tool to exert forms of concertive (collective) control over employees and their development

The outcome of my research agrees with Kim's (2010) assertion that training activities that are strategically handled could act as an employee motivational tool that contributes to improved organizational performances as well as improved productivity and job satisfaction (Sahinidis \& Bouris, 2008). This is because some employees may see these training interventions as potential opportunities for developing their career and expertise, which ultimately has a beneficial influence on the overall organizational employee retention figures (Kim, 2010).

\subsubsection{OI Marker: Vertical Communication and Clarity of Organizational Purpose}

Depth of vertical communication, which is synonymous to the flow of fact and uncoerced information from those occupying the owner-manager position(s) to employees within SMEs, is crucial to the employees' OI process, as employees see this as a symbol of trustworthiness (i.e. transparency) within the company. This is in resonance to the Bartels et al. (2010) argument that dimensions of vertical communication are an important predictor of organizational identification. 
According to Bartels et al. (2010), an employee's identification with an organization does not depend primarily on the quantity of contact with immediate colleagues within a work group but more on the quality and frequency of communication from and with top management. Thus, vertical communication serves as a foundational element in the development of $\mathrm{OI}$ in many cases, but $\mathrm{Ol}$ can develop without it. However, vertical communication when active in small businesses affords a more effective (or swifter) basis for favourable EOI statuses within the company.

The research participants were also of the opinion that this transparency is often linked to how these owner-manager(s) clarify the organizational purpose of the company to the employees from the start of employment. This resonates with Cheney's (1983) assertion about the need for communication of organizational goals to employees, without which employees might not be able to form any bond with the company. A benefit to management of engaging in this transparency activity is that it helps employees to identify more swiftly their 'fit' within the company. That is, they are able to traverse their employee OI process more quickly and easily and ultimately find their Ol locus within the context of their firm. One benefit of this echoed by the respondents is that it often results in improved overall company productivity.

\subsubsection{OI Marker: Ethical Management and Leadership}

The notion of ethical management and leadership practices as an Ol Marker is viewed from the point of being a strong motivational tool influencing an employee's organizational identification process within SMEs. That is, the managers and leaders of small businesses were also the owners in the cases of about $70 \%$ of the total interview respondents, actors commonly known as 'owner managers' (David, 2013), and play a major role in the OI process of employees. Employees see the concept of management and leadership as encompassing feelings of 'respect', 'trust' and 'parent/older-sibling figure' interrelationship. 
Respect from their company's management and leadership was viewed as a feeling of being valued or being recognised by owner-managers or management in general. Trust was seen in terms of reliability in the actions undertaken or to be undertaken by this party, while presence of a parent/older-sibling figure could generate a sense of admiration for this, often owner-manager, management figure. All these facets work together to create employees who identify more with their respective SME companies.

Research respondents expressed that having feelings of being respected and trusted by their organizational leadership contributed significantly to the way they construe belongingness with the company. Respect they defined as being a function of admiration for their personality and the work they do within the business, whereas trust was seen as a function of being able to take ownership of their respective roles within the business. That is, their ability to carry out their daily operational responsibilities with little or no supervision from an owner-manager figure. These two elements may be seen as notions of reciprocity: 'I trust them' and 'they trust me' ideas expressed by an employee to the owner-manager or management and viceversa. This was instrumental in formation of a strong interrelation bond between these actors within the small business.

The parent/older-sibling figure perspective they explained as a show of emotion or care towards employees by the owner-managers. It is a somewhat protective instinct that relevant employees feel their owner-managers may display or exhibit towards them, which generates a strong feeling of belongingness towards their companies. This is especially pertinent since these actors of parent/older-sibling figures are the company owners and essentially represent the highest leadership or managerial levels within the businesses. This perspective as expressed by some of the research respondents was also affirmed and demonstrated by their perception about their organizations in general. 
For example, when asked to describe the identity of their organizations they used words such as Compassionate; Supportive; Staff-focused; Hierarchical Structural Friendly; Warm; Welcoming; Open to ideas; Good; Inclusive; Conducive Environment; and Trustworthy, all of which suggest caring, emotional and affective feelings on the part of these employees for their respective organizations. Part of the care respondents' iterated owner-managers demonstrated included taking their physical and emotional wellbeing seriously as well as their professional advancement. A particular respondent emphasized her gratitude to her ownermanager boss for sponsoring some of her professional exams she had to sit, to help develop her professional acumen further.

\subsubsection{OI Marker: Company Image/ldentity and Culture}

The perceived company image is often representational of the EOI or EOD exhibited by SME employees. Analysis and interpretation of interview data suggest that when asked to characterise the identities of the company during the interview process, SME employees viewed their company's identity or image and culture in a certain light and characterised them with different word qualifiers such as: Competent; Efficient; Compassionate; Supportive; Customer-focused; Staff-focused; Hierarchical Structural Uniformity; Effective Performance Management; Builds Staff knowledgeGap; Friendly; Warm; Welcoming; Open to ideas; Good; Inclusive; Conducive Environment; and Trustworthy. All these aforementioned factors reflect their perceptions of the external image of the company.

The organizational culture of a small business is another important factor that influences the Ol process of its employees. Explaining this, research participants linked the organizational or corporate culture with the company image and identity. Employees perceive the identity of the company to be a reflection and function of the culture within the firm. While the majority of the fifteen respondents assessed the culture of their respective organizations by using positive comments in most areas, a proportion also identified areas where their companies were lagging behind. 
Previous organizational cultural studies detailed such cultures to be observable organizational behaviours that members' exhibit, as they share common ideological perceptions with members of such organizations. For example, one of the research respondents described her organization as welcoming and stated that management aims to create a total person who is capable and equipped for a work role in the organization by providing an enabling environment. This she perceived through her colleagues and managers providing the supportive environment she needs, which could be linked to management adopting an ethical management and leadership approach.

This perspective is in alignment with the Bergami and Bagozzi (2000) finding that 'perceived organizational prestige has positive effects on commitment by organizational identification mediating effect'. That is, factors such as attractiveness, distinctiveness, prestige, and construed external image have been classically construed to be major predictors of employee Ol statuses (Dukerich et al.,2002; Dutton, et.al., 1994). This is based on the finding that employees are more likely to identify with organizations that have an attractively perceived external organizational image since this often acts to boost employees' own self-image (Podnar, Golob \& Jancic, 2011; Farrell \& Oczkowski, 2012).

Podnar, Golob and Jancic (2011) argued that this perceived external prestige is a component of the organizational and corporate identity employees feel their organizations possess and this identity is often a reflection of the organizational culture of these companies. That is, organizational identity and employees' perception of their status influence EOI; hence, when an organization is perceived to be more attractive, employees are more likely to develop stronger identification status. Thus, company identity and culture are other key OI Markers highlighted in the stories of the research participants as influencing employees' OI status in small business organizations. 


\subsubsection{OI Marker: Company Productivity}

Company productivity is another factor that research participants identified as influencing their Ol statuses within their respective firms. Expatiating on this, respondents saw the overall reported productivity of the company as an indicator of their own productivity and successes. They emphasised that the productivity they observe or are made aware of ultimately makes them feel either more satisfied or dissatisfied with the company, thereby impacting on their perceived Ol status within these companies. This could also be related to the vertical communication Ol Marker, since if they are not made aware of the company's overall productivity levels they may not be motivated to identify with the organization. Thus, reflecting on the companies' productivity indices acts as a positive reinforcement of EOI statuses within such companies.

\subsection{Organizational Identification Employee Situational-gap Markers}

The other findings from the OI markers category, aside from the training interventions discussed earlier on in this chapter, interpreted as the research respondents' psychological interpretation of own identity relative to their respective firm, are detailed as follows:

\subsubsection{OI Marker: Facilitated Training Interventions}

The facilitated aspect of training interventions is a component of the employee situational-gap OI Marker category in the CRF and this section addressed in part the $R Q_{3}$. The primary explanation for this type of training from the narratives of the research respondents is that small businesses can sometimes be supportive of their career development by facilitating training opportunities external to the firm. In the respondents' view, this means they are committed to developing them as a total person to the extent that even when the organization does not organise nor run the respective training activities itself, it often still voluntarily supports such individual initiatives. Employees are often compensated for such training by being given benefits such as time-off in lieu for time spent on external training, as well as 
increased responsibility on completion of such programmes to transfer and implement what has been learnt to the job role. Hence, their sense of Ol within the company is increased. For further clarity, respondents asserted this notion to training programmes that are beneficial to their overall career aspirations and thus have strong probability of improving their individual productivity within the firm despite not being mandatory trainings programmes. These was particularly true for interview participants with high-skilled professional roles within their respective SMEs.

\subsubsection{Ol Marker: Work Relationships}

Maintaining healthy work relationships is a key contributor to having EOI in small businesses. Research respondents reflected on the values they place on their varying work relationships (which could range from employee-employee, manageremployee, to customer-employee) and identified such interrelationships as influential for their Ol process within the company. A highly-valued work relationship often translated to better EOI, while work relationships on a lower value scale that are pertinent to employees' day to day job can be indicative of EOD status within the company. Conversely, lower-valued work-relationships related to employeeemployee and manager-employee interrelationships that are not essential to respondents' day to day routines do not necessarily have the same effect on their OI processes. A good customer/client-employee work relationship also contributes somewhat to improving employees' self-confidence and thus their Ol levels within the firm.

For instance, employees are likely to be more satisfied with their job roles when they have good interrelationships with colleagues or managers with whom they work in close proximity on a routine basis, compared to those with work colleagues they interact with less frequently. This is viewed from a behavioural and emotional point of view by the research participants. That is, the nature of interrelationships between employees and other employees or owner-managers/ management of their business organization is a crucial factor in determining their Ol statuses within the business. 


\subsubsection{Ol Marker: Personalised benefits}

Personalised benefits employees derived from their small business organizations also play a significant part in influencing their EOI or EOD status within their respective firms. Employees in small businesses often exhibited such personalised benefits as a function of the strategic fit between the organization's goals and objectives and the employee's own individual career aspirations. This outcome emphasises the Gratton et al. (1999) assertion that discerned level of strategic fit facilitates competitive advantage. This process creates individualistic behaviour within an organization which comprises individual aspirational elements, with respondents articulating this in terms of their life-long aspirations, dreams and their professional or career aspirations.

This attainment of personalised benefits within the firm is somewhat linked to the notion of organizational sacrifice surfacing from the analytic outcomes of the initial pilot study conducted during this research process. Though this conceptual notion or sacrifice was not strongly prominent within the actual research interview narratives, it might be worthwhile as a site of future research to explore the possibility of employees exhibiting an active trade-off or opportunity cost in terms of sacrificing their personalised benefits for developing their EOI or EOD statuses within the SMEs.

That is, personalised benefits, though a precursor of Ol status in small business organizations, are not often seen as an indicative factor on the Ol marker spectrum, suggesting that it becomes latent or its significance dormant as employee $\mathrm{OI}$ statuses develop within the small businesses studied. In contrast, in the pilot study conducted at an earlier stage of this research, organizational sacrifice seemed to take centre-stage in relation to the $\mathrm{OB}$ construct under discussion. This apparent difference in both studies observed may be associated with the differences in sectoral distribution of respondents from both the pilot and main study as while 
respondents were from only SME for the pilot study, the main study comprised of a more diversified sectoral distribution of respondents.

\subsubsection{OI Marker: Perceived In-company Worth}

Another contributory factor to the employee's OI process is their 'sense of incompany worth'. The research participants expressed this as a function of their incompany responsibilities and how seemingly important these responsibilities are to the products and services provided to company customers. They affirmed that the more salient they felt their roles, responsibilities and/or duties within the small business to be to the company's daily activities or set routine targets undertaken, the more their sense of in-company importance or worth developed. Respondents noted that an improvement in their perceived self-worth within the company would lead to a corresponding improvement or increase in their EOI statuses within the company.

Respondents felt that when their sense of worth within the small business is significantly visible and significantly important to other actors' job duties and roles, such as those of their colleagues, managers and subordinates, their perceived incompany worth also increases. This ultimately is expected to increase their identification statuses and the job duties they mentioned are often specialised job responsibilities that sometimes no one else can carry out within the business. This means that they consider themselves to be highly valuable to the business holistically.

\subsection{Key Research Contributions to Academic Knowledge}

Primarily, this research study has contributed to the academic body of knowledge in a cross-disciplinary manner, through integration of the Organizational Behaviour (OB) and HRM (specifically HRD) study areas. While much extant literature has focused on the productivity aspects in small businesses (Valadez, 2011) and its importance to their survival, this research has focused on identifying organizational behavioural issues pertinent to the performance of employees in such businesses 
who themselves are integral to the operationalisation of activities of these small businesses.

This research inductively helped develop an understanding of $\mathrm{Ol}$ from small businesses employee perspectives within the business management study area, which to the best of my knowledge has not been done previously. In this task the study also combined the IPA methodological approach and sense-making theories of Weick and Dervin, neither of which have previously been used in the business management field of study, to achieve its key aim of clarifying the influence of training activities on employee belongingness exhibited with the organization, i.e. employee OI.

In summary, the revised CRF (figure 9, p. 203) suggests that intra-organizational affective factors known as Ol employer situational-context markers exert an influence on the employee Ol process within SMEs and these factors are often outside the control or power of employees, while affective cognitive factors known as OI employee situational-gap markers and within the control of employee also influence the OI process of employees in organizations.

For example, some research participants argued that while they cannot control what their organizations vertically communicate to them (that is communication from the management level within the organizations to lower level employees), they do tend to have a better sense of belonging with the organization when the company is clear about its goals, plans and expectations, especially regarding the company's business focus and direction per time. This viewpoint is in resonance with the Bartels et al. (2012) rationale that the vertical communication climate influences organizational identification.

This lends nuance to $\mathrm{OB}$ literature as to ascertaining how employees in business organizations may behave when exposed to the identified organizational elements 
such as vertical communication and ethical leadership as previously shown in figure 4 (p. 82) and depicted in figure 9 of this research conclusion and recommendations chapter (p. 203).

An example of an Ol employee situational-gap marker would be personalised benefits such as employees' career aspirations. Interviewed employees highlighted that their personal and professional aspirations affect how they see themselves within the organization, especially in relation to what they think they are able to get from the small business in pursuit of achieving this aspiration.

Another key difference between the initial CRF and the revised CRF is in the conceptualisation of how organizational identification as an organizational behavioural attribute is developed or exhibited by small business employees. That is, how $\mathrm{OI}$ is measured as an employee attribute. Post interview analysis of research participants' stories suggested that each employee always has a measurable level of Ol at any point in time as a company employee. This understanding is further substantiated through their narrated stories suggesting that SME employees do not exhibit neutral OI status; instead, they only exhibit OI statuses that vary within the vectoral range of EOI to EOD depicted in the revised CRF (Figure 9, p. 203).

Thus, while my research outcomes affirm the existence of employee organizational disidentification status, they dis-affirm the existence of neutral or ambivalent employee OI status within a small business organization. Therefore, this marks progression from the most recent ideological perspectives on the conceptualisation of OI suggesting the existence of three main Ol status categories: disidentification, ambivalent and neutral Ol statuses (Dukerich et al., 1998; Elsbach \& Bhattacharya, 2001; Kreiner \& Ashforth, 2004). Outcomes from my research suggest Ol occurs as a vectoral construct with no absolute null levels. That is, SME employees exhibit OI status with a measurable value or quantity and do not develop neutral or detached identification or a dual identification status represented as the ambivalent 
identification status explained in extant literature (Kreiner \& Ashforth, 2004: refer to section 2.5.1, pp. 30 to 33 ).

The key argument from my research outcomes relative to previous OI studies thus relates to the way organizational status is exhibited or displayed by employees in small businesses.

Previous scholars identified cases where a person neither connects his or her identity with nor separates it from the organization, or, at the extreme, does not even have an opinion or knowledge about the organization, as neutral identification (Dukerich, Kramer \& Parks 1998; Kreiner \& Ashforth, 2004). A state of neutral identification, which I believe to mean a detached identification status, is also said to be a state of "non-existent or broken identifications" (Pratt, 1998). In essence, if it is a non-existent identification status, then it cannot be said to be relative to Ol itself as an OB construct, since, arguably, Ol status is a function of observable organization identification signs or behaviours evident in an individual employee within the firm. My research results suggest that once individuals become employees of a small business, they automatically begin to exhibit varying levels of $\mathrm{Ol}$ which are mainly determined by the factors called OI markers within the context of my research. For example, responses from the research participants suggested that they either disidentify with their company or have a varying level of identification with their company, but never an indifferent attitude. That is, they either feel a sense of belonging with their organizations that could vary in depth or do not feel any sense of belonging, which is a disidentification OI status.

Ambivalent identification, on the other hand, as defined in extant OI literature refers to the existence of a dualised state of employee identification and disidentification statuses in organizations (Kreiner \& Ashforth, 2004). This is said to occur when an employee can identify with certain dimensions or traits of their organization's identity, or has simultaneous identification and disidentification with the same traits (Kreiner \& Ashforth, 2004). Cases attributed to this include incompatibility of the organization's 
identity with that of the individual and where the image of the organization is negative (Kreiner \& Ashforth, 2004; Elsbach \& Bhattacharya, 2001).

Evidence from my research outcomes suggests that this idea of a dual status is not viable. Although the stories of some of the research participants highlight certain areas of the organizational culture with which they were not entirely happy, they viewed OI has a holistic construct. That is, they do not feel or see the need to single out various attributes of their company culture to define their sense of belonging with their company, but rather their Ol status reflects their generalised feelings of belonging with or attractiveness of their companies. For example, they do not perceive their companies in both a negative and positive light simultaneously but rather depict their company's identity with certain words that reflect their overall perception of the company.

This assertion moves the instability argument of ambivalent identification (Festinger 1957 \& Heider, 1958, both cited in Boros, 2008) further, suggesting it to be an unstable and unsafe state of mind. It certainly adds unnecessary layers of complexity to the primary notion of identification, especially as identification is often seen as a function of mapping an organization's identity over an individual's own identity. Thus, suggesting an ambivalent identification would mean suggesting an employee would also have a dual personality within the context of the organization. On this basis, my research outcome fits more rationally with the notion that 'ambivalent identification' as proposed in extant literature is more likely a disidentification OI status. This status essentially is characterised as employees defining themselves through attributes opposing those used in defining their small business organizations. This status is thus reflected in the revised CRF presented in figure 9 as EOD status: point $A$ represents the most extreme point in the Ol status range that employees within the firm can occupy.

The notion of disidentification was developed from Dutton, Dukerich, and Harquail (1994) who defined it as the degree to which a person defines him or herself as not 
having the same attributes that define the organization. Disidentification is said to occur when individuals do not identify with the organization or have "a self-perception based on a cognitive separation between one's identity and one's perception of the identity of an organization, and a negative relational categorisation of oneself and the organization" (Elsbach \& Bhattacharya, 2001). This means that a disidentified employee feels a dissonance with the organization's identity. Employees with EOD status often feel distanced from their companies and they are often said to maintain "a sense of self-distinctiveness through perceptions and feelings of disconnection" (Elsbach \& Bhattacharya, 2001). That is, they identify self by forming a sort of cognitive dissociation from the small business organization, which results in the employees seeing their own identity as superior to and fundamentally different from that of their organizations.

Furthermore, the outcomes of my research affirms some of the antecedents of disidentification and conversely identification identified by Elsbach and Bhattacharya (2001), including the following: the perception that one's personal values conflict with the values of the organization - relates to the personal benefits element of the $\mathrm{OI}$ employee situational-gap marker category; the perception that an organization's reputation might affect own social identity - relates to the company image/identity and culture element of the OI employer situational-context marker category; and perception of the organization that is based on a lack of personal experience with the organization or its members - relates to work relationships, ethical management and leadership, vertical communication and clarity of organizational purpose, and training intervention components of the revised CRF( figure 9, p.203)

Consequences of organizational disidentification, according to research respondents, include feelings of job dissatisfaction, low self-esteem, as well as increased intention to leave the company in pursuit of one's own career development and aspirations. Also, the respondents' narratives aroused significant debate related to varying trust and respect levels, especially within employee-employer relationships. Owner- 
managers are seen as a major determinant of employee OI status within SMEs based on the nature of relationship they cultivate with their employees.

Furthermore, although the results of this OI study primarily indicate that OI status of employees within small business, when measured at a set point in time, is a vector function with a magnitude (quantity) as well as a directional element, the measurable value of the status can fluctuate. These differences in degree have however not been researched in depth due to limitations encountered during the research journey (these are discussed in more depth later on in section 5.8, pp 224 to 226). Thus, I recommend this area of $\mathrm{Ol}$ study as a worthwhile site for future study.

As indicated earlier on in this chapter, OI markers within the context of small businesses are categorised into two main divisions, either as employer situationalcontext markers or employee situational-gap markers. Whereas employer situationalcontext markers are indicators of intra-organizational drivers of $\mathrm{Ol}$, which are often beyond the control of employees, employee situational-gap markers are OI markers that are representational of an SME employee's psychological interpretation of their own identity relative to the firm, which is primarily within their control. Interpretation of both categories is nevertheless situated within an employee's sense-making framework, further suggesting that there are elements of employer-employee powerplay variability present within SMEs. That is, the OI process is influenced by differing interactions between the elements of the two OI markers categories and the resulting employee Ol status reflected at a set point in time is therefore a function of the prominent $\mathrm{OI}$ Marker category at that time. For example, the employer's power is seemingly more dominant in determining an employee's Ol status when positively perceived employer situational-context $\mathrm{OI}$ markers are prominently operational within the organization, while the reverse is the case when the employee situational-gap OI markers are perceived to be more prominent.

The construction of the two Ol Marker categories as indicated in the CRF is supported by understanding of sense-making theories in extant literatures. These 
theories argue that sense-making can actually be an observable behaviour which can have both cognitive and procedural or affective elements which are expressed as internal and external characteristics respectively (Dervin, 1983). Possession of these two components allows individual possessors (researcher and research participants, in the context of this research) to construct and design their movement through the time-space context. This suggests that sense-making is a process, often involving energy-releasing components such as force, power, and constraint. That is, sense-making of an unknown construct is a function of both individualised attributes such as internal motivation, resistance and possible help received from others as well as of external environmental sources or conditions external to the individual (Savolainen, 2006).

This process creates a dichotomous boundary, where humans seeking informational clarity are constrained by structural power forces (the organizational management in the context of this research) and individual principles which can sometimes challenge organizational norms and are thus seen as sites of power themselves. This dichotomous boundary is formed between the employer situational-context OI markers and employee situational-gap OI markers within the context of my study. Hence, employing the function of the sense-making theory helped to effectively situate and objectively understand the OI construct in this exploratory research.

In addition to the above, sense-making models are also based on an assumption that the understanding of an objective reality can be tempered by the observer or perceiver's sense-making process (Weick, 1993) and suggest that integration can only be achieved through a continuous and flexible negotiation between the self and the organization in context (Scott et al.,1998). Thus, both categorisations of power sites in organizations have significant importance in understanding organization identification as an $\mathrm{OB}$ phenomenon. 
Therefore, the simultaneous existence and operation of both categories of $\mathrm{Ol}$ markers within a firm relative to an employee's sense-making framework may cause the loci of the employee's OI to continuously fluctuate, resulting in variations in the $\mathrm{OI}$ level at different times. However, at any set time, an employee's OI status has a measurable value within the defined OI vector range. These defined interactions are presented in the subsequent sub-section within the revised CRF (figure 9, p.203).

Sectoral differences among the employees interviewed can perhaps offer further insight into employees' OI statuses. My textual data analysis indicated that respondents working in the Health, Financial and Food industries generally affirmed higher OI statuses, while respondents within the Retail and Social Care sectors indicated the lowest feelings of belongingness with their current SME workplace. Possible explanations for this finding could not be explored in detail within the remit of my research but this would appear to be a good site for further explanatory OI research.

Additionally, research respondents that were more highly skilled seemed to demonstrate higher EOI statuses in comparison to respondents that worked in lowskilled roles within their companies (section 4.6, p. 194). Thus, this factor could potentially provide indication to the management of small businesses as to the OI statuses they could expect from employees in different roles within their firms.

\subsection{Research Policy-Practice Implications for Small Business Organizations}

The underlying importance of research work in general is the ability to inform tangible organizational practices and this research is no exception. The research findings supported the argument of the researcher that training interventions can influence employees' OI processes. In addition, other employee OI markers within SMEs were also highlighted, all of which have policy-practice implications for small business organizational practices from the researcher's perspective. Thus, these policypractice implications noted as recommendations are detailed in this subsection. 
The researcher recommends that:

- Small businesses should incessantly explore the significant role of training interventions in strengthening the behavioural 'belongingness' of their employees by initiating need-tailored training activities in-company.

- Such need-tailored training activities are best computed and initiated via consultation with the potential trainees themselves, i.e. SME employees.

- Facilitating external training activities can also improve the level of commitment small businesses can get from their employees. This does not necessarily involve giving monetary rewards or contributions, especially as funding is often argued to be in short supply in SMEs (Brown \& Lee, 2014); instead, it can be a strategy used to foster a company culture through aiding the overall development of the individual. Giving individuals' time off work, in lieu of time spent on off the job trainings initiated and undertaken by the employee, as well as providing viable avenues for the practice of relevant newly acquired skills within the company are some ways SME employers can incorporate this culture into their businesses. These practices are likely to gradually craft a better sense of belonging, increasing the organization's influence within employees' sense-making mind frame and thus improving their Ol statuses within the organization.

- In order to develop strong Ol among their employee's small business organizations need to be explicit about their business aims and purpose at the point of employing staff. It is nevertheless acknowledged that SMEs tend to be continuously engaged in a process of growth, until maturity or death is reached, which often means that their business aims and purpose are constantly changing as well. One way of overcoming this obstacle is through active encouragement of vertical communication which is a twoway, up and down flow of information among the management and 
employees of organizations. Emphasising the importance of this company culture during new staff members' initial induction into the company will likely help foster a healthy and thriving business.

- Another recommendation is for small businesses to incorporate ethical management and leadership practices. Again, due to their often significantly reduced access to funds in comparison with large companies, this can often be difficult for small businesses in practice. Moreover, SMEs are often profit driven to the exclusion of all else. However, small business employees are likely to be more productive when they feel respected, can trust their employers to provide elements of stability and reliability, and when they experience a sense of admiration from their leadership. These aforementioned longings of small business employees can be tied to provision of an emotional wall of support by their managers during their duration within the organization.

- Small business owners or managers should be meticulous about allocation of roles and duties to their employees. Owner-managers in their managerial responsibility need to ensure employees skills set are matched to the duties they perform on the job which should help boost their jobsatisfaction and thus productivity. This could ultimately lead to development of an increased in company self-worth and hence increased employee Ol status. Thus such small businesses stand the chance of gaining highly proactive and productive staff members which is on the long run is beneficial for the company with returns such as low employee turnover rates and increased employee loyalty.

- Lastly, healthy working relationships are crucial to a healthy business organization. Proactively designing, facilitating and implementing 
workplace policies that foster good working relationships among staff members and customers, across all hierarchical levels in the company, should be a priority for small business owner-managers. For productivity and innovation to be predominant within the company, there needs to be a conducive business environment, as facilitated and represented by its people.

\subsection{Research Limitations}

This sub-section summarises the limitations that emerged in the course of this study. These include issues related to the sampling choice, time limitations, and generalisability of research findings.

\subsubsection{Development of Sampling Frame}

The population frame for this research is employees within small businesses (Miles and Huberman, 1994) in the UK. Restricting the sampling to this said population essentially introduces bias, because in this study the Ol subject under scrutiny, as construed from extant literature, is viewed as a psychological construct underpinned by sense-making theoretical models and other SME actors could have been selected as alternative research subjects, i.e. the owner-managers.

However, given the scope of the research, time limitations as well as the perceived integral importance of employees to SME businesses as the major drivers of productivity (Oriaku, 2012), the researcher made a choice to limit the choice of respondents to the purposely selected employees with homogenous characteristics such as previous participation in training activities, underpinned by the adapted IPA method procedural norms (Smith \& Flowers, 1998).

The researcher also endeavoured to select research respondents from across geographically distant regions of the UK (Londo,; West Midlands, North East), as well as different sectors (Health and Social Care; Food; Financial; IT; Retail and Education) as far as possible to encourage expression of diverse opinions and 
richness in the respondents' narratives. Thus, the research objectives and questions were significantly relevant to the circumstances of each selected research participant, which enabled collation of detailed and comprehensive textural data for $y$ analysis.

In retrospect, however, the researcher would suggest further testing of the conceptual model across more viable sample frames across the UK as well as inclusion of owner-managers' perspectives in future research on the Ol subject. This will be explained further in the subsequent section on further research areas.

\subsubsection{Time Limitations}

The interviews conducted as the source of data during this research represented the reflective narratives of the respondents relative to the OI construct under discussion. Given more time, it would have been fruitful in terms of possibly producing richer and more in-depth information if a longitudinal study had been conducted wherein after a set time a second round of interviews was scheduled with the initial research participants to ascertain whether or not their viewpoints expressed in their initial stories had changed or not. This would have helped substantiate and possibly influenced the dynamics of the OI markers identified from this research study.

Although time constraints did not permit the researcher to do this, nonetheless, the research outcome still contributes to the ensuing discussion of $\mathrm{Ol}$ within the small business organizational context. Furthermore, strong consideration is being given to furthering this line of research post-doctorate.

\subsubsection{Generalisability of Research Findings}

Major criticisms of qualitative interpretative studies relate to the small sample size and inability to replicate professed study outcomes in larger samples and population (Mays \& Pope, 1995). This research sample cannot avoid this criticism, since the sample size of fifteen interview respondents, on the surface, seemingly represents a minute proportion of the total research population, i.e. SME employees in the UK. 
However, having followed the rationale for validity of interpretative studies, this research contributes some novel findings to the existing body of literature on the $\mathrm{OI}$ construct within the organizational behavioural study area. Analysis of the narratives of the study respondents provided an in-depth pool of understanding as to the influence of intra-organizational and individualised elements on employees' OI status in small businesses.

The study was able to scaffold, thematically and interpretatively, the markers of OI within the sense-making framework of the research participants using a conceptual framework initially developed from notions embedded in OI extant literature. Locating and redefining these $\mathrm{OI}$ markers post-thematic analysis was significantly pertinent to how OI development should be construed in the future by organizational practitioners. Thus, if put into practice this final framework could significantly influence how people management strategies are implemented within SMEs in the UK.

\subsubsection{EOD Identification Status in SMEs}

A notable finding by my study was the limited development of an absolute EOD status as inferred from the respondents, as while some showed elements of dissatisfaction with their current working arrangements, the general consensus and trend in the research respondents' textual data was possession of Ol statuses more skewed towards the EOI end of the OI vector categorisation rather than EOD. As a researcher I took careful steps to avoid introduction of bias into my research; however, I must accept that there is a possibility of this attribute being only specific to the characteristics of the research sample used in my study. Hence more analytical outcomes in relation to EOD statuses and hence belongingness may have been generated from my study if the study sample had been larger. 


\subsection{Literature Publication Emanating from the Research Thesis}

The researcher is currently working on an article for publication in HRM/D based journal publications based on the developed CRF in my study as well as submitting working papers at the UFHRD (a conference I regularly attend) 2019 conference focused on articulating the impact of the parent/older-sibling factor in cultivating EOI or EOD statuses in small businesses.

\subsection{Future Areas of Organizational Identification Research}

Considering the scope of this research, which is exploratory in nature within the small business organizational context and relative to the OI notion, it opens up new viable areas of future OI research, especially since this construct has not previously been adequately explored within the business management area of study.

In addition to the above contribution, it combined the procedural dictates of the IPA research methodology with Wieck and Dervin's sense-making models to define its perspective and focus, which has not been done before within a business management research context.

Thus to conclude this thesis, a number of areas for further research are highlighted in the subsequent final sub-sections of this thesis.

\subsubsection{Exploration of the Owner-manager OI Perspectives on SMEs}

This research explored the notion of Ol from the narratives of SME employees, putting strong emphasis on the interpreted meanings of their stories. It may be argued that their perspective is prone to bias or skewed and offers an imbalanced view relative to the Ol markers put forward. Thus, it is feasible to suggest that engaging owner-managers in the discussion might give a more balanced and seemingly fairer picture of how the OI process is construed and developed within the context of small businesses. This is important as owner-managers are also very active actors within this organizational context. 
Thus, the researcher proposes that further research be done in relation to this. It could consist of a study comparing heterogeneous samples of employees and owner-managers or could be a comparative study across these two primary actors within the small business context.

\subsubsection{Introducing a longitudinal time study perspective to the OI research context within SMEs}

Another suggestion for possible future research would be to conduct a longitudinal study, where the instrument for data collection could be applied at more than one interval across the defined time-length of the research, e.g. through recurring scheduled interview sessions. Since the primary data collected from the respondents is their recounted stories, it would be of interest to explore whether the interpretative meanings of these recounted stories are subject to change across specified time intervals.

Introducing a longitudinal perspective into the research context may or may not result in a shift in the outcomes of the research. This means that over time, the viewpoints of the respondents as to what they construe Ol to be, what influences their Ol status and how training impacts on their OI status may be subject to change. This may also ultimately have an impact on the overall research outcome as well as the applicability of the revised conceptual framework in this context.

\subsubsection{Exploration of the expression of Organizational Sacrifice relative to OI research in an SME context}

A third area of possible future research relates to exploration of some of the themes that emerged in the pilot study that were not so apparent in the main research interview analysis, including factors such as the notion of organizational sacrifice versus perseverance and forced/mandatory training.

These factors were not highlighted from the narratives of the main research respondents and it would be interesting to explore why these factors were not 
present and what could have been responsible for their transition from being active participants in the pilot study outcomes to being absent from the main research outcomes. While the answer to this is uncertain, since this was not explored in detail within the context of this research, there might be some differences across differing sectors that may influence the outcome of $\mathrm{Ol}$ status in business management studies. The pilot study focused primarily on respondents from only one sector of the UK economy, so this mono-sectoral approach may have been responsible for the themes generated from the pilot study results. Thus, future research could conduct a comparative study to ascertain if cross-sectoral dynamics may be responsible for this significant impact on the outcomes generated from the main research study.

\subsubsection{Exploration of the Magnitude attribute of OI construct as a vector function in SMEs}

A final probable site of future study proposed by the researcher is explorative study to determine how the magnitude attribute of the Ol construct expressed as a vector function by this research participants can be measured. That would entail developing a deterministic scale to ascertain how the identified Ol markers influence the magnitude of their Ol status per time from point $A$ to $B$ as illustrated in the revised research conceptual framework, within the respective SME context.

It would be interesting to categorise or grade the impact of each factor by gaining an understanding of which factors have more pronounced or more subtle influences on the employees' OI status within the businesses. That is, which OI Marker can be said to have the most impact in determining the different levels of Ol statuses of employees within small businesses. For example, the study may seek to expand on determining which $\mathrm{OI}$ markers influence higher $\mathrm{EOI}$ statuses closer to point $\mathrm{B}$ or those lower down and closer to point $A$ and therefore synonymous to EOD statuses in small business organizations as shown in the revised CRF. 


\section{BIBLIOGRAPHY}

Abimbola, T., \& Vallaster, C. (2007). Brand, Organizational Identity and Reputation in

SMEs: An Overview Qualitative Market Research: An International Journal 10(4), 341-348

Abrams, D., \& Hogg, M. (1999). Social Identity and Social Cognition. Oxford: Blackwell.

Abrams, D., Ando, K., \& Hinkle, S. (1998). 'Psychological Attachment to the Group: Cross-Cultural Differences in Organizational Identification and Subjective Norms as Predictors of Workers' Turnover Intentions,' Personality and Social Psychology Bulletin, 24, 1027-1039.

Adekunle, A. (2011). Impact of Online-Employee Training on Operational Risk Management (Master's Thesis) University of East London

Albert, S. \& Whetten, D.A. (1985). "Organizational Identity", in Cummings,L.L. \& Staw, B.M.(Eds), Research in Organizational Behavior, , JAI Press, Greenwich, CT, 7, 263-295

Albert, S., Ashforth, B.E.\& Dutton, J.E. (2000). 'Organizational Identity and Identification: Charting new waters and building new bridges'. Academy of Management Review, 25(1), 13-17

Alvesson, M. \& Wilmott, H. (2002). Identity Regulation as Organizational Control: Producing the Appropriate Individual'. Institute of Economic Research Working Paper Series

Ancona, D. (2011). Sensemaking: Framing and Acting in the Unknown. In S. Snook, N. Nohria \& R. Khurana (Eds) The Handbook of Teaching Leadership: Knowing, Doing, and Being (3-20) LA: Sage 
Appelbaum, E., Bailey, T., Berg, P., \& Kalleberg. A.L. (2000). Manufacturing advantage: Why high performance work systems pay off. Ithaca, NY: Cornell University Press.

Aronson, J., Blanton, H., \& Cooper, J. (1995). From dissonance to disidentification: Selectivity in the self-affirmation process. Journal of Personality and Social Psychology, 68, 986-996.

Ashforth, B.E., \& Mael, F. (1989). 'Social Identity Theory and the Organization,' Academy of Management Review, 14, 20-39.

Aust, P. (2004). Communicated values as indicators of organizational identity: A method for organizational assessment and its application in a case study. Communication Studies, 55(4), 515-534.

Bacaksiz, F. E., Tuna, R., \& Seren, A. K. H. (2017). 'The Relationships between Organizational Identification, Job Performance, and Job Crafting: A Study Among Nurses' International Journal of Caring Sciences, 10(1), 251-259

Barney, J. (1991). Firm Resources and Sustained Competitive Advantage, Journal of Management 17(1), 99-120

Bartels, J., Peters, O., de Jong, M., Pruyn, A., \& van der Molen, M., (2010). "Horizontal and vertical communication as determinants of professional and organizational identification", Personnel Review, 39(2), 210-226

Bartels, J., Pruyn, A., \& de Jong, M. (2009). 'Employee Identification Before and After an Internal Merger: A Longitudinal Analysis,' Journal of Occupational and Organizational Psychology, 82, 113-128

Becker, B.E, \& Huselid, M.A. (1998). 'High performance work systems and firm performance: a synthesis of research and managerial implications'. Research in Personnel and Human Resource Management, 16, 53-101 
Becker, B.E, \& Huselid, M.A. (2006). 'Strategic human resource management: Where do we go from here?'. Journal of Management, 32(6), 898-925.

Becker, T.E., Billings, R.S., Eveleth, D.M., \& Gilbert, N.L. (1996). Eoci and bases of employee commitment: Implications for job performance. Academy of Management Journal, 39, 464-482

Bergami, M., \& Bagozzi, R. P. (2000). Self-categorization, affective commitment and group self-esteem as distinct aspects of social identity in the organization. British Journal of Social Psychology, 39, 555-577.

Biggerstaff, D., \& Thompson, A.R. (2008). 'Interpretative Phenomenological Analysis (IPA): A Qualitative Methodology of Choice in Healthcare Research'. Qualitative Research in Psychology, 5(3), 173-183

Blau, P. (1964). Exchange and power in social life. New York: Wiley

Boland, R. J. Jr. (1984). Sense-making of Accounting Data as a Technique of Organizational Diagnosis. Management Science, 30(7), 868-882

Bomber, E. M., \& lyer, V.M. (2002). Big 5 Auditors' Professional and Organizational Identification: Consistency or Conflict. A Journal of Practice \& Theory, 21(2), 21-38.

Boros, S. (2008) Organizational Identification: Theoretical and Empirical Analyses of Competing Conceptualizations, Cognition, Brain \& Behaviour, 12(1), 1-27

Boroş, S., \& Curseu, P. (2005). Psychological contracts in work relations. Some data regarding the psychometric properties of the PSYCONES questionnaire on a Romanian sample. Revista de Psihologie Organizationala, 5, 123-145.

Bowen, D., \& Ostroff, C. (2004). Understanding HRM-firm performance linkages: The role of the "strength" of the HRM system. Academy of Management Review, 29, 203-221. 
Boydell, T. (1971). A Guide to the Identification of Training Needs, London: BACIE.

Bradley, D. B., \& Cowdery, C. (2004). Small Business: Causes of Bankruptcy assessed 11-12-2017

at

http://www.unife.it/economia//m.economia/insegnamenti/governance-and-accountingof-smes/materiale-didattico/lecture-1/reading-1 bradley-and-cowdery

Brammer, S., Millington, A., \& Rayton, B. (2007). 'The Contribution of Corporate Social Responsibility to Organizational Commitment,' The International Journal of Human Resource Management, 18, 1701-1719.

Brannick, T., \& Coghlan, D. (2007). In defense of Being "Native": The case for Insider Academic Research. Organizational Research Methods, 10(1). 59-74

Bressler, M. S. (2012). How Small Businesses Master the Art of Competition through Superior Competitive Advantage. Journal of Management \&Marketing Research 11, $1-12$

Brief, A. P. (1998). 'Attitudes in and Around Organizations'. Thousand Oaks, CA: Sage.

Brown, A.D. (1995), Organisational Culture, Pitman Publishing, London.

Brown, A. D. (2006). A Narrative Approach to Collective Identities. Journal of Management Studies, 43(4), 731-753

Brown, R., \& Lee, N. (2014) Funding Issues Confronting High Growth SMEs in the UK. Research Committee ICAS, Edinburgh, UK.

Brown, M. E., Trevino, L. K., \& Harrison, D. A. (2005). Ethical leadership: A social learning perspective for construct development and testing. Organizational Behavior and Human Decision Processes, 97(2), 117-134. 
Bryman, A., \& Bell, E. (2003). Business Research Methods. 2nd edn. Oxford: Oxford University Press.

Bullis, C., \& Bach, B. (1989). Socialization turning points: An examination of change in organizational identification. Western Journal of Speech Communication, 53, 273293.

Carlin, L., End, C. M., \& Mullins, M. E. (2010). 'Validating Kreiner and Ashworth's Organizational Identification Measure in an Engineering Context'. Journal of Applied HRM 12(1), 75-83

Carmeli, A., \& Freund, A. (2002). The Relationship between Work and Workplace Attitudes and Perceived External Prestige. Corporate Reputation Review, 5(1), 51 68.

Carmeli, A., Gilat, G., \& Weisberg, J. (2006). Perceived External Prestige, Organizational Identification and Affective Commitment: A Stakeholder Approach. Corporate Reputation Review, 9(1), 92-104.

Carmeli, A., Gilat, G., \& Waldman, D. A. (2007). The role of perceived organizational performance in organizational identification, adjustment and job performance. Journal of Management Studies, 44, 972-992.

Campbell, J.W., \& Im, T. (2014); Identification and Trust in Public Organizations: A Communicative Approach. Public Management Review 17(8) 1-20

Chapman, E., \& Smith, J.A. (2002). 'Interpretative Phenomenological Analysis and the New Genetics'. Journal of Health Psychology 7(2) , 125-130

Charted Institute of Personnel Development (2019). "Introduction" in Strategic Human Resource Management accessed on 31/01/2019 at https://www.cipd.co.uk/knowledge/strategy/hr/strategic-hrm-factsheet\#6738 
Chaudron, D. (1996). 'Training Effectiveness-Don't Overload the Horse' , HR Focus, January, 10-11.

Chen, G., \& Klimoski, R. J. (2003). The Impact of Expectations on Newcomer Performance in Teams as Mediated by Work Characteristics, Social Exchanges, and Empowerment. Academy of Management Journal, 46(5), 591-607.

Cheney, G., \& Christensen, L. T. (2001). Organizational identity: Linkages between internal and external communication. In F. M. Jablin \& L. L. Putnam (Eds.), New handbook of organizational communication, (231-270), Thousand Oaks, CA: Sage.

Cheney, G., \& Tompkins, P.K. (1987). Coming to Terms with Organizational Identification and Commitment. Central States Speech Journal, 38, 1-15

Cheney, G. (1983). On the various and changing meanings of organizational membership: A field study of organizational identification. Communication Monographs, 50, 342-362.

Chowdhary, N., \& Saraswat, B. P. (2004). Service leadership study. Journal of Services Research, 3(2), 105-123.

Ciftcioglu, A. (2010). Exploring the Role of Perceived External Prestige in Employee's Emotional Appeal: Evidence from a Textile Firm, Business and Economics Research Journal, 1(4), 85-96

Collings, D. G., \& Wood, G. (2009). 'Human Resource Management: A critical approach' in 'Human Resource Management: A critical approach' Eds Collings, D. G. and Wood, G. London: Routledge

Correia, M. C., Rebelo dos Santos, N., \& Passmore, J. (2016). Understanding the Coach-Coachee-Client Relationship: A Conceptual Framework for Executive Coaching. International Coaching Psychology 11(1), 6-23

Cresswell, J. W. (2009). Research Design: Qualitative, Quantitative and Mixed Approaches $3^{\text {rd }}$ Edn. Thousand Oaks CA: Sage 
Crotty, M. (1998). The foundations of social research: Meaning and perspective in the research process. Thousand Oaks, CA: Sage

Crotty, M. (2003). The foundations of social research: Meaning and perspective in the research process. $3^{\text {rd }} \mathrm{Edn}$. Thousand Oaks, CA: Sage

David, H. (2013). The Reluctant Employer: An Exploration of the First Employment Decisions and Early Employment Experiences of Small Business Owner-Managers. Doctoral thesis: University of Gloucestershire.

de Chernatony, L., \& Cottam, S. (2008). Interactions between Organisational Cultures and Corporate Brands. Journal of Product \& Brand Management, 17(1), 1324

De Cremer, D., \&Tyler, T.R., (2005) Managing Group Behavior: The Interplay between Procedural Justice, Sense of Self, and Cooperation. Advances in Experimental Social Psychology 37,151-218

Delbridge, R., \& Edwards, T. (2013). Inhabiting Institutions: Critical Realist Refinements to Understanding Institutional Complexity and Change. Organization Studies 34(7), 927-947

Delery, J.E., \& Doty, D.H. (1996). 'Modes of theorizing strategic human resource management: Tests for universalistic, contingency and configurational performance predictions'. Academy of Management Journal, 39(4), 802-835

Denzin, N., \& Lincoln, Y. (eds.) (2003). Collecting and Interpreting Qualitative Materials.2nd ed. Thousand Oaks: Sage.

Denzin, N. (1994) 'The Art and Politics of Interpretation', in N. K. Denzin and Y. S. Lincoln (eds) Handbook of Qualitative Research, (500-515). Thousand Oaks, CA: Sage. 
Department for Business Innovation and Skills (2016). Business Population Estimates for UK and regions UK government 2016 viewed at www.gov.uk

Department for Business, Energy, and Industrial Strategy (2017). Business Population Estimates for UK and regions UK government viewed at www.gov.uk

Dervin, B. (1983). An overview of sense-making research: Concepts, methods and results to date. Paper presented at the International Communication Association Annual Meeting, Dallas, TX.

Dervin, B. (1992). From the mind's eye of the 'user': The sense-making qualitativequantiatative methodology. In J.D. Glazer \& R.R. Powell (Eds.), Qualitative research in information management (61-84). Englewood: CO: Libraries Unlimited.

Dervin, B. (1993). Verbing communication: mandate for disciplinary invention. Journal of Communication, 43(3), 45-54.

Dervin, B. (1994). Information democracy: An examination of underlying assumptions. Journal of the American Society for Information Science, 45(6), 369385.

Dervin, B. (1998). Sense-making theory and practice: An overview of user interests in knowledge seeking and use. Journal of Knowledge Management, 2(2), 36-46.

Dervin, B. (1999). Chaos, order, and sense-making: A proposed theory for information design. In R. Jacobson (Ed.), Information design (35-57). Cambridge, MA: MIT Press

Dervin, B. (2003). Sense-making's journey from metatheory to methodology to method: An example using information seeking and use as research focus. In Dervin, B., \& Foreman-Wernet, L. \& Lauterbach, E., (Eds.), Sense-Making Methodology Reader. Selected writings of Brenda Dervin (133-163). Cresskill, NJ: Hampton Press. 
Doosje, B., Ellemers, N., \& Spears, R., (1995). Perceived intragroup variability as a function of group status and identification. Journal of Experimental Social Psychology, 31, 410-436

Downs, C. W., \& Adrian, A. D. (2004). Assessing organizational communication: Strategic communication audits. New York: Guilford Press

Dukerich, J. M., Golden, B. R., \& Shortell, S. M. (2002). Beauty is in the eye of the beholder: The impact of organizational identification, identity, and image on the cooperative behaviors of physicians. Administrative Science Quarterly, 47, 507-533.

Dukerich, J. M., Kramer, R. M., \& Parks, J. M. (1998). The dark side of organizational identification. In D. A. Whetten, \& P. C. Godfrey (Eds.), Identity in organizations: Building theory through conversations (245-256). Thousand Oaks, CA: Sage

Dutton, J.E., \& Dukerich, J.M. (1991). 'Keeping an Eye on the Mirror: Image and Identity in Organizational Adaptation,' Academy of Management Journal, 34, 517554.

Dutton, J.E., Dukerich, J.M., \& Harquail, C.V. (1994). 'Organizational Images and Member Identification,' Administrative Science Quarterly, 39, 239-263.

Dwyer, S.C., \& Buckle, J.L. (2009). The Space Between: On Being an InsiderOutsider in Qualitative Research. International Journal of Qualitative Methods, 8(1), 54-63

Easterby-Smith, M., Thorpe, R., \& Jackson, P.R. (2008). Management Research. Los Angeles, London: Sage Publications.

Easterby-Smith, M., Thorpe, R., \& Lowe, A. (2012). Management Research: An Introduction, $4^{\text {nd }} \mathrm{Ed}$, London: Sage Publications

Economic and Social Research Council (2015). Framework for Research Ethics. London: ESRC 
Edwards, M.R., \& Peccei, R. (2007). "Organizational identification: development and testing of a conceptually grounded measure", European Journal of Work and Organizational Psychology, 16(1), 25-57

Eggins, R. A., O'Brien, A. T., Reynolds, K. J., Haslam, S. A., \& Crocker, A. S. (2008). Refocusing the focus group: AlRing as a basis for effective workplace planning. British Journal of Management, 19, 277-292.

Ellemers, N., \& van Rijswijk, W. (1997). Identity needs versus social opportunities: The use of group-level and individual- level identity management strategies. Social Psychology Quarterly, 60, 52-65.

Ellemers, N., de Gilder, D., \& Haslam, S. A. (2004). Motivating individuals and groups at work: A social identity perspective on leadership and group performance. Academy of Management Review, 29, 459-478.

Ellemers, N., Kortekaas, P., \& Ouwerkerk, J. W. (1999). Self-categorisation, commitment to the group and group self-esteem as related but distinct aspects of social identity. European Journal of Social Psychology, 29, 371-389

Ellemers, N., Spears, R., \& Doosje, B. (2002). Self and social identity. Annual Review of Psychology, 53, 161- 186.

Elsbach, K. D., \& Bhattacharya, C. B. (2001). Defining who you are by what you're not: Organizational disidentification and the National Rifle Association. Organization Science, 12, 393-413.

Epitropaki, O., \& Martin, R. (2005). The moderating role of individual differences in the relation between transformational/transactional leadership perceptions and organizational identification. The Leadership Quarterly, 16, 569-589

European Union (2003). European Union Commission Recommendation of 6 May 2003 on the definition of micro, small and medium-sized enterprises (notified under 
document number $C$ (2003) 1422) (2003/361/EC) In The new SME definition User guide and model declaration Enterprise and Industry Publications

Farooq, O., Rupp, D., \& Farooq, M. (2017). Multiple Pathways through which Internal and External Corporate Social Responsibility influence Organizational Identification and Multifoci Outcomes: The Moderating Role of Cultural and Social Orientations. . Academy of Management Journal 60(3), 954-985

Farrell, M.A., \& Oczkowski, E. (2012). Organizational Identification and Leader Member Exchange Influences on Customer Orientation and Organizational Citizenship Behaviours. Journal of Strategic Marketing 20(4), 365-377

Finlay, L. (2009). Debating Phenomenological Research Methods. Phenomenology and Practice, 3(1), 6-25

Fiol, C. M., Pratt, M. G., \& O'Connor, E. J. (2009). Managing intractable identity conflicts. Academy of Management Review, 34, 32-55.

Fisher, C. (2004) Researching and Writing a Dissertation for Business Students. London: Pearson Education

Fombrun, C. (1996) Reputation: Realizing Value from the Corporate Image, Harvard Business School Press, Boston. MA.

Fombrun, C., \& Shanley, M., (1990). What's in a Name? Reputation Building and Corporate Strategy. Academy of Management Journal, 33(2), 233-258

Fox, H. L. (2013). Strategic Human Resource Development in Small Businesses in the United States, Academy of Entrepreneurship Journal, 19(1), 77-118.

Gadamer, H.G. (1977). Philosophical Hermeneutics. Berkeley, Los Angeles and London: University of California Press. 
Gioia, D. A., \& Thomas, J.B. (1996). Institutional Identity, Image and Issue Interpretation: Sensemaking during Strategic Change in Academia. Administrative Science Quarterly, 41, 370-403.

Gioia, D. A., Schultz, M., \& Corley, K (2000). Organizational Identity, Image and Adaptive Instability. Academy of Management Review 25, 63-82

Goldhaber, G.M. (1993). Organizational communication. $6^{\text {th }}$ edn. New York: McGrawHill.

Gratton, L., Hope-Hailey, V., Stiles, P., \& Truss, C. (1999). Linking individual performance to business strategy: The people process model'. Human Resource Management, 38(1), 17-31

Greene, M. J. (2014). On the Inside Looking IN: Methodological Insights and Challenges in Conducting Qualitative Insider Research. The Qualitative Report, 19(15), 1-13

Guba, E. G., \& Lincoln, Y. S. (1994). Competing paradigms in qualitative research in N.K. Denzin and Y.S. Lincoln (Eds) Handbook of qualitative research (105-117) Thousand Oaks, CA: Sage

Guest, G., MacQueen, K. M., \& Namey, E. E. (2012). Applied thematic analysis. Thousand Oaks, CA: Sage.

Hackman, J. R., \& Oldham, G. R. (1980). Work Redesign, Reading, MA: AddisonWesley

Hankinson, P., \& Hankinson, G. (1999). Managing Successful Brands: An Empirical Study which compares the Corporate Cultures of Companies Managing the World's Top 100 Brands with those Managing Outsider Brands. Journal of Marketing Management, 15, 135-55. 
Hansen, S.D., Dunford, B.B., Boss, A.D., Boss, R.W., \& Angermeier, I. (2011) 'Corporate Social Responsibility and the Benefits of Employee Trust: A CrossDiscipline Perspective'. Journal of Business Ethics,102(1), 29-45

Hanson, J. (2013). Educational developers as researcher: the contribution of insider research to enhancing understanding of role, identity and practice. Innovations in Education and Teaching International, 50(4), 388-399.

Harris, F., \& de Chernatony, L. (2001). Corporate branding and corporate brand performance European Journal of Marketimng, 35 (3/4), 441-456

Haslam, S. A. (2001). Psychology in organizations: The social identity approach. London: Sage.

Haslam, S. A., Postmes, T., \& Ellemers, N. (2003). More than a Metaphor: Organizational Identity Makes Organizational Life Possible British Journal of Management, 14, 357- 369

Hatch, M. J., \& Schultz, M. (1997). "Relations between organizational culture, identity and image", European Journal of Marketing, 31, 356-365.

He, H., \& Baruch, Y. (2010). Organizational identity and legitimacy under major environmental changes: Tale of two UK building societies. British Journal of Management 21 (1), 44-62.

He, H., \& Brown, A. D. (2013). Organizational identity and organizational identification: A review of the literature and suggestions for future research. Group and Organization Management, 38 (1), 3-35.

Heidegger, M. (1978). Being and Time. Oxford: Basil Blackwell.

Hekman D. R., Bigley G. A., Steensma H. K., \& Hereford J. F. (2009). Combined effects of organizational and professional identification on the reciprocity dynamic for professional employees. Academy of Management Journal 52(3), 506-526. 
Hellriegel, D., Slocum, Jr .J. W., \& Woodman, R.W (2001). Organizational Behavior: 9th Ed. Ohio: South-Western College Publishing

Hogg, M.A., \& Abrams, D. (1988). Social Identifications: A Social Psychology of Intergroup Relations and Group Processes, London: Routledge

Holloway, I., \& Wheeler, S. (1996). Qualitative Research for Nurses. Oxford: Blackwell Scientific

Humphreys, M., \& Brown, A.D. (2002). Narratives of Organizational Identity and Identification: A Case Study of Hegemony and Resistance. Organization Studies, 23, 421-447.

Humphreys, M., \& Brown, A.D. (2008). An Analysis of Corporate Social Responsibility at Credit Line: A Narrative Approach. Journal of Business Ethics, 80(3), 403-418.

Humphrey, A. (2012). Transformational Leadership and Organizational Citizenship Behaviors: The Role of Organizational Identification. The Psychologist-Manager Journal, 15, 247-268

Huselid, M.A. (1995). The Impact of Human Resource Management Practices on turnover, Productivity and John. A. W. and James W. Cartada (2002) Training and Performance Year Book: McGraw-Hill Companies, Inc.

Hutagalung, I. (2016). Selective Exposure and Consumer Behavior: Interpretative Phenomenological Analysis in Consumer Behaviour of $Z$ Generation Adolescent on the Ad Information of Smartphone Selection, International Journal of Organizational Innovation, 9(2), 97-104

Iqbal, S. M. J., Nabi, A. A., Muneer, S., \& Anuar, M. A. (2013). Impact of Training on Expectation of Employee and Employer: A comparative study, Information Management and Business Review 5(12), 601-607 
Jacobs, R.L., \& Jones, M.J. (1995). Structured On-the-Job Training:Unleashing Employee Expertise in the Workplace". Performance Improvement Quarterly, 9(2), 97-100.

Jaiswal, P., Gupta, M., \& Singh, V.P. (2014). Employee Attitude towards Training and Development Practices in Manufacturing Sector: A Case Study. Proceedings of 4th National Conference on Human Resource Management, Review of HRM, 3, 228-235 Johnson, P., \& Clarke, M. (2006). 'Mapping the terrain: an overview of business and management research methodologies' in Johnson, P. and Clarke, M. (eds.) Business and Management Research Methodologies. London: Sage

Jones, J. P., Nast, H., \& Roberts, S. (1997). Thresholds in Feminist Geography: Difference, Methodology, Representation. New York: Rowman and Littlefield.

Kabat-Zinn, J. (2004). Wherever you go, There you are: Mindfulness Meditation for Everyday Life. London: Piatkus

Kim T., Chang K., \& Jae-Ko J. (2010). Determinants of organizational identification and supportive intentions. Journal of Marketing Management, 26(5-6), 413-427

Kim, J. (2010). Strategic Human resource practices: Introducing alternatives for organization performance improvement in the public sector. Academy of Management Executive, 7(2), 38-49

Kohler, T., Landis, R. S., \& Cortina, J. M. (2017). Establishing Methodological Rigor in Quantitative Management Learning and Education Research: The Role of Design, Statistical Methods, and Reporting Standards. Academy of Management Learning \& Education 16(2), 173-192

Kozlowski, S. W. J., \& Salas, E. (1997). A multilevel organizational systems approach for the implementation and transfer of training. In J. Kevin Ford (Ed.), Improving training effectiveness in work organizations, 247-287. 
Kreiner, G. E., \& Ashforth, B. E. (2004). Evidence toward an expanded model of organizational identification. Journal of Organizational Behaviour, 25, 1-27.

Kuchinke, K. P. (1995). Managing learning for performance. Human Resource Development Quarterly, 6(3), 307-316.

Langdridge, D. (2007). Phenomenological Psychology: Theory, Research and Method. Harlow, England, London, New York: Pearson Education Limited.

Larson, G., \& Tompkins, P. (2005). Ambivalence and resistance: A study of management in a concertive control system. Communication Monographs, 72(1), 121.

Laverty, S.M. (2003). Hermeneutic Phenomenology and Phenomenology: A Comparison of historical and Methodological Considerations. International Journal of Qualitative Methods, 2(3), 21-35

Leavy, P. (ed) (2014). The Oxford handbook of qualitative research. New York, NY, US: Oxford University Press.

Leavy, P. (2016). Essentials of Transdisciplinary Research: Using Problem-Centered Methodologies. New York: Routledge.

Leavy, P. (2017). Research Design: Quantitative, Qualitative, Mixed Methods, Artbased and Community-Based Participatory Research Approaches New York: Guilford Press

LeCompte, M.D., \& Preissle, J., with Tesch, R. (1993). Ethnography and qualitative design in educational research 2nd edn. San Diego, CA: Academic Press

Lee, G. \& McGuiggan, R. (2008). Understanding Small- and Medium-Sized Firms' Financial Skill Needs. Journal of International Finance \& Economics, 8(3), 93-103. 
Lincoln, Y.S. \& Guba, E.G. (1985) Naturalistic Inquiry. Thousand Oaks, California, London, New Delhi: Sage

Liu, W., Zhu, R., \& Yang, Y. (2010). I warn you because I like you: Voice behavior, employee identifications, and transformational leadership. Leadership Quarterly, 21, 189-202

Mael, E.A., \& Ashforth, B.E. (1995). Loyal from day one: Biodata, organizational identification, and turnover among newcomers. Personnel Psychology, 48, 309-333

Mael, F., \& Ashforth, B.E. (1992). 'Alumni and Their Alma Mater: A Partial Test of the Reformulated Model of Organizational Identification,' Journal of Organizational Behavior, 13(3), 103-123.

Mason, J. (2002). Qualitative Researching .2nd edn. London: SageMays, N., \& Pope, C. (1995) Rigour and Qualitative Research. British Medical Journal, 311 (6997), 109112.

McBain, R. \& Parkinson, A. (2017). Placing Relationships in the Foreground: The Role of Workplace Friendships in Engagement. In: Zerbe, W. J., Hartel, C. E. J., Ashkanasy, N.M., \& Petitta, L. (eds.) Emotions and Identity. Research on Emotion in Organizations, 13, (199-221) Bingley: Emerald Publishing Limited

McAllister, D.J. (1995). Affect and Cognition Based Trust as Foundations for Interpersonal Cooperation in Organizations. Academy of Management Journal, 38(1), 24-59

Meyer, J. P., Allen, N. J., \& Smith, C. A. (1993). Commitment to Organizations and Occupations: Extension and Test of a Three-Component Conceptualization. Journal of Applied Psychology, 78(4), 538-551.

Meyer, J., \& Allen, N. (1991). A three-component conceptualisation of organizational commitment. Human Resource \& Management Review, 1, 61-89. 
Meyer, J.P., \& Allen, N.J. (1997). Commitment in the workplace: Theory, research and application. Thousand Oaks, CA: Sage.

Meyerson, D., \& Martin, J. (1987). Cultural Change: An integration of three different views. Journal of Management Studies 24(6), 624-647

Mezirow, J. (1997). Transformative learning: Theory to practice. In P. Cranton (Ed.). Transformative learning in action New Directions in Adult and Continuing Education, 74, 5-12. San Francisco: Jossey-Bass

Mignonac, K., Herrbach, O., \& Guerrero, S. (2006). The Interactive Effects of Perceived External Prestige and Need for Organizational Identification on Turnover Intentions. Journal of Vocational Behavior, 69, 477-493.

Miles, M., \& Huberman, A. (1994). Qualitative Data Analysis. London: Sage

Noe, R. A. (1999). Employee training and development Boston, MA: Irwin/McGrawHill.

O'Reilly, C. A., Chatman, J., \& Caldwell, (1991). Organizational identity. Journal of Management Inquiry, 15, 119-234.

Oriaku, N. (2012). Current Challenges Facing Small Businesses: Case of Brazil and United States, International Journal of Finance and Policy Analysis, 4(2), 16-25

Owens, P.L. (2006). 'One More Reason Not to Cut Your Training Budget: The Relationship between Training and Organizational Outcomes', Public Personnel Management, 35(2), 163-172.

Patton, M.Q. (2002) Qualitative Research and Evaluation methods. $3^{\text {rd }}$ edn Thousand Oaks, CA: Sage

Peters, K., Haslam, S. A., Ryan, M. K., \& Fonseca, M. (2012). Working With Subgroupo Identities to Build Organizational Identification and Support for 
Organizational Strategy: A Test of the ASPIRe Model. Group \& Organization Management, 38(1), 128-144.

Peterson, D.K. (2004). The Relationship between Perceptions of Corporate Citizenship and Organizational Commitment. Business and Society; Sep 2004; 43(3), 296-319

Pfeffer, J. (1994). Competitive advantage through people: Unleashing the Power of the Work Force. Boston: Harvard University Press

Podnar, K., Golob, U., \& Jancic, Z. (2011). Identification with an organization as a dual construct. European Journal of Marketing 45(9/10), 1399-1415.

Podsakoff, P.M., MacKenzie, S.B., Paine, J.B., \& Bachrach, D.G. (2000). Organizational Citizenship Behaviors: A Critical Review of the Theoretical and Empirical Literature and Suggestions for Future Research. Journal of Management, 26, 513-563.

Porter, W. L., Steers, R. M., Mowday, R. T., \& Boulian, P. V. (1974). Organizational commitment, job satisfaction, and turnover among psychiatric technicians. Journal of Applied Psychology, 59, 603-609.

Pratt, M. G. (1998). To be or not to be? Central questions in organizational identification. In D. A. Whetten \& P. C. Godfrey (Eds.), Identity in organizations: Building theory through conversations, 171-208. Thousand Oaks, CA: Sage.

Pratt, M. G. (2000). The good, the bad, and the ambivalent: Managing identification among Amway Distributors. Administrative Science Quarterly 45(3): 456-493

Preuss, L., Haunschild, A., \& Matten, D. (2009). The rise of CSR: Implications for HRM and Employee Representation. The International Journal of Human Resource Management, 20(4), 953-973 
Ravasi, D., \& Phillips, N. (2011). Strategies of Alignment: Organizational Identity Management and Strategic Change at Bang \& Olufsen. Strategic Organization 9(2), 101-135

Rawlings, E., \& Cowell, N. (2015). Educational psychologists' experience of taking part in group supervision: A phenomenological study. Educational \& Child Psychology. 32(3), 51-64

Research Development Framework (2010) University of Gloucestershire

Riantoputra, C. D. (2010). Know Thyself: Examining Factors That Influence the Activation of Organizational Identity Concepts in Top Managers' Minds. Group \& Organization Management 35(1), 8-38

Ricoeur, P. (1981). Paul Ricoeur hermeneutics and the human sciences. (Thompson, J. B., Ed.). New York: Cambridge University Press

Riketta, M. (2005). 'Organizational Identification: A Meta-Analysis,' Journal of Vocational Behavior, 66, 358-384.

Riketta, M., \& van Dick, R. (2005). Foci of attachment in organizations: A metaanalytic comparison of the strength and correlates of workgroup versus organizational identification and commitment. Journal of Vocational Behavior, 67, 490-510.

Ritchie J., \& Spencer L. (1994). Qualitative data analysis for applied policy research. In Bryman A, \& Burgess R, editors. Analysing qualitative data. London: Routledge; 173-194.

Ritchie J., Lewis, J., \& Elam, G. (2003). in Maruster, L. \& Gijsenberg, M.J. (2013) eds. Qualitative Research Methods. New Delhi: Sage; 79-110.

Robson, C. (2002). Real World Research, $2^{\text {nd }}$ Ed, Oxford: Blackwell 
Rode, V., \& Vallaster, C. (2005). "Corporate branding for start-ups: the crucial role of entrepreneurs", Corporate Reputation Review, 8(2), 121-135.

Rodrigo, R. P., \& Arenas, D. (2008). Do employees care about corporate social responsibility? A typology of employees according to their attitudes. Journal of Business Ethics, 83(2), 265-283.

Rolfe, G. (2006). Validity, trustworthiness and rigour: quality and the idea of qualitative research. Methodological Issues in Nursing Research. 304-310

Rousseau, D. M. (1998). Why workers still identify with organizations. Journal of Organizational Behavior, 19, 217-233.

Roy, A., \& Raymond, L. (2008). 'Meeting the training needs of SMEs: Is e-Learning a Solution?', The Electronic Journal of E-learning, 6 (2), 89-98.

Rupp, D.E., Ganapathi, J., Aguilera, R.V., \& Williams, C.A. (2006), 'Employee Reactions to Corporate Social Responsibility: An Organizational Justice Framework,' Journal of Organizational Behavior, 27, 537-543.

Sahinidis, A.G., \& Bouris, J. (2008). 'Employee perceived training effectiveness relationship to employee attitudes', Journal of European Industrial Training, 32 (1), 63-76.

Santhanam, N., Kamalanabhan, T.J., \& Dyaram, L. (2014). Examining the Moderating Effects of Organizational Identification between Human Resource Practices and Employee Turnover intention in Indian Hospitality Industry. BizStrategy: $4^{\text {th }}$ Annual International Conference on Business Strategy and Organizational Behaviour , 76-84

Saunders, M., Lewis, P., \& Thornhill, T. (2012). Research Methods for Business Students. London, New York: Prentice Hall. 
Savolainen, R. (2006). Information Use as Gap-Bridging: The Viewpoint of SenseMaking Methodology, Journal of The American Society for Information Science and Technology, 57(8), 1116-1125

Savvides, N., Al-Youssef, J., Colin, M., \& Garrido, C. (2014). Journeys into Inner/Outer Space: reflections on the methodological challenges of negotiating insider/outsider status in international educational research. Research in Comparative and International Education, 9(4), 412-425

Schaubroeck, J. M., Peng, A. C., \& Hannah, S. T. (2013). Developing trust with peers and leaders: Impacts on organizational identification and performance during entry. Academy of Management Journal, 56(4), 1148-1168

Schein, E. H. (1984). Coming to a New Awareness of Organizational Culture. Sloan Management Review. 25(2), 3-16.

Schuler, R.S., \& MacMillan, I.C. (1984). Gaining Competitive Advantage through Human Resource Management Practices. Human Resource Management, 23(3), 241-255.

Scott, C.R., Corman, S.R., \& Cheney, G. (1998). Development of a Structurational Model of Identification in the Organization. Communication Theory, 8, 298-336

Scott, S.G., \& Lane, V.R. (2000). 'A Stakeholder Approach to Organizational Identity,' Academy of Management Review, 25, 43-62.

Senge, P. M. (1990). The Fifth Discipline-The Art and Practice of the Learning Organization. London: Random House.

Siegel, P. H., \& Sisaye, S. (1997). An Analysis of the Difference between Organization Identification and Professional Commitment: A Study of Certified Public Accountants. Leadership \& Organization Development Journal, 18(3), 149-165 
Sillince, J. A. A., \& Brown, A. D. (2009). Multiple Organizational Identities and Legitimacy: the Rhetoric of Police Websites. Human Relations, 62(12), 1829-1856.

Silverman, D. (2006). Interpreting Qualitative Data. London: Sage Publications.

Singh, N.A. (2016). A phenomenological exploration of the holistic experiences of Bangladeshi MBA students at a UK University. Doctoral thesis: University of West London.

Smidts, A., Pruyn, A.H., \& van Riel, C.B.M. (2001). The impact of employee communication and perceived external prestige on organizational identification. Academy of Management Journal, 49(5), 1051-1062.

Smith, J.A, Jarman, M., \& Osbourne, M. (1999). Doing Interpretative Phenomenology Analysis. In Murray, M \& Chamberlain K. (Eds.), Qualitative Health Psychology: Theories and Methods. London: Sage.

Smith, J.A. (2004). Reflecting on the Development of Interpretative Phenomenological Analysis and its Contribution to Qualitative Research in Psychology. Qualitative Research in Psychology 1, 39-54.

Smith, J., \& Dunsworth, F. (2003). Qualitative methodology. In Valsiner, J. \& Connolly, K. (Eds.), Handbook of Developmental Psychology. London: Sage.

Smith, J.A., \& Osborn, M. (2003), "Interpretative phenomenological analysis", in Smith, J.A. (Ed.), Qualitative Psychology: A Practical Guide to Research Methods, Sage, London, 51-80.

Smith, J. A., Flowers, P., \& Osborn, M. (1997). Interpretative Phenomenological Analysis and the Psychology of Health and Illness. In Yardley L., (Ed.), Material Discourses of Health and Illness (68-91). Florence: Routledge. 
Smith, J.A. (2007) Hermeneutics, Human Sciences and Health: Linking Theory and Practice. International Journal of Qualitative Studies on Health and Well-being, 2, 311

Smith, J.A. (2008). Qualitative Psychology: A Practical Guide to Research Methods 2nd edn. London: sage.

Smith, J.A., Flowers, P., \& Larkin, M. (2009). Interpretative Phenomenological Analysis: Theory, Method and Research. London: Sage Publications.

Starbuck, W. H., \& Milliken, F. J. (1988). Executives' perceptual filters: What they notice and how they make sense. In D. C. Hambrick (Ed.). The executive effect: Concepts and methods for studying top managers (pp. 35-65). Greenwich, CT: JAI Press

Steele, C. M. (1988). The Psychology of Self-Affirmation: Sustaining the Integrity of the Self. In L. Berkowitz (Ed.). Advances in Experimental Social Psychology, 21, 261302. New York: Academic Press

Stutchbury, K., \& Fox, A. (2009). Ethics in Educational Research: Introducing a Methodological Tool for Effective Ethical Analysis. Cambridge Journal of Education, 39(4), 489-504

Tajfel, H. (1978). 'Social Categorization, Social Identity, and Social Comparison,' in Differentiation Between Social Groups: Studies in the Social Psychology of Intergroup Relations, ed. H. Tajfel, London: Academic Press, 61-76.

Tajfel, H. (1982). Social Psychology of Intergroup Relations. Annual Review of Psychology, 33, 1-39.

Teel, K.S., Verdeli, H., Wickramaratne, P., Warner, V., Vousoura, E., Haroz, E.E., \& Talati, A. (2016). 'Impact of a Father Figure's Presence in the Household on Children's Psychiatric Diagnoses and Functioning in Families at High Risk for Depression'. Journal of Child and Family Studies. 25(2), 588-597 
Thietart, R.A., \& Wauchope, S. (2001) Doing Management Research, London: Sage

Tompkins, P.K., \& Cheney, G. (1983). Account Analysis of Organizations: Decision Making and Identification. In L.L. Putnam \& M.E. Pacanowsky (Eds.), Communication and Organizations: An Interpretive Approach (123-146). Beverly Hills, CA: Sage.

Top, S (2013). In the Strategic Sense, Evaluating Transactional and Relational Expectations as Reflection of Emotional Contract. Procedia - Social and Behavioral Sciences, 99, 230-239

Torbrand, P., \& Ellam-Dyson, V. (2015). The Experience of Cognitive Behavioural Group Coaching with College Students: An IPA Study Exploring its Effectiveness. International Coaching Psychology Review, 10(1), 76-93.

Tsoukas, H. (1989). The Validity of Idiographic Explanations. Academy of Management Review, 14(4), 551-561

Tsui, P. Y., \& Ngo, H. Y. (2015). A Study of Organizational Identification of Faculty Members in Hong Kong Business Schools. Journal of Education for Business, 90, 427-434

Turner, J.C, Hogg, M.A., Oakes, P.J., Reicher, S.D., \& Se Wetherell, M.S. (1987). Rediscovering the Social Group: A Self-categorization Theory. Oxford: Blackwell,

Tyler, T. R., \& Blader, S. (2000). Co-operation in Groups: Procedural Justice, Social Identity and Behavioral Engagement. Philadelphia: Psychology Press.

Valadez, R. M. (2011). The Value Proposition of Small Businesses: Economic Engines for Job Creation. Journal of Management \& Marketing Research, 9,1-11.

Valdiserri, G. A., \& Wilson, J. L. (2010). The Study of Leadership in Small Business Organizations: Impact on Profitability and Organizational Success', The Entrepreneurial Executive, 15, 47-71. 
van Dick, R. (2001). Identification in Organizational Contexts: Linking Theory and Research from Social and Organizational psychology. International Journal of Management Reviews, 3(4), 265-283

van Dick, R., Christ, O., Stellmacher, J., Wagner, U., Ahlswede, O., Grubba, C., Hauptmeier, M., Hohfeld, C., Molzen, K., \& Tissington, P.A. (2004), 'Should I Stay or Should I Go? Explaining Turnover Intentions With Organizational Identification and Job Satisfaction,' British Journal of Management, 15, 351-360.

van Dick, R., Wagner, U., Stellmacher, J., \& Christ, O. (2004). The Utility of a Broader Conceptualization of Organizational Identification: Which Aspects Really Matter? Journal of Occupational and Organizational Psychology, 77. 1-20.

van Dick, R., Wagner, U., Stellmacher, J., \& Christ, O. (2005). Category Salience and Organizational Identification. Journal of Occupational and Organizational Psychology, 78, 273-285.

van Knippenberg, D., \& Sleebos, E. (2006). Organizational Identification Versus Organizational Commitment: Self Definition, Social Exchange, and Job Attitudes. Journal of Organizational Behavior. 27, 571-584.

van Knippenberg, D., \& van Schie, E.C.M. (2000). 'Foci and Correlates of Organizational Identification,' Journal of Occupational and Organizational Psychology, 73, 137-147.

van Manen, M. (2007). Researching Lived Experience: Human Science for an Action Sensitive Pedagogy. Ontario: The Althouse Press.

Wainwright, D., \& Sambrook, S. (2010). The Ethics of Data Collection: Unintended Consequences? Journal of Health Organization and Management, 24(3), 277-287

Walliman, N. (2006). Social research methods. London: SAGE publications. 
Walumbwa, F. O., \& Hartnell, C. A. (2011). Understanding Transformational Leadership-Employee Performance Links: The Role of Relational Identification and Self-Efficacy. Journal of Occupational and Organizational Psychology, 84(1), 153172.

Walumbwa, F. O., Avolio, B. J., \& Zhu, W. (2008). How Transformational Leadership Weaves its Influence on Individual Job Performance: The Role of Identification and Efficacy Beliefs. Personnel Psychology, 61(4), 793-825.

Walumbwa, F. O., Mayer, D. M., Wang, P., Wang, H., Workman, K., \& Christensen, A. L. (2011). Linking Ethical Leadership to Employee Performance: The Roles of Leader-Member Exchange, Self-Efficacy, and Organizational Identification. Organizational Behavior and Human Decision Processes, 115(2), 204-213.

Waterman, R. H., Jr. (1990). Adhocracy: The Power to Change. Memphis, TN: Whittle Direct Books.

Waterson, P., Clegg, C.W., Bolden, R., Pepper, K., Warr, P.B., \& Wall, T.D. (1999). The Use and Effectiveness of Modern Manufacturing Practices: A Survey of UK Industry. International Journal of Production Research, 37, 2271-2292.

Way, S.A. (2002). High Performance Work Systems and Intermediate Indicators of Firm Performance within the US Small Business Sector. Journal of Management, 28, 765-785.

Weick, K. E. (1988). "Enacted Sensemaking in Crisis Situations," Journal of Management Studies, 25, 305-317.

Weick, K. E. (1993). The Collapse of Sensemaking in Srganizations: The Mann Gulch disaster. Administrative Science Quarterly, 38, 628-65

Weick, K. E. (1995). Sensemaking in Organizations. Sage Publications, Thousand Oaks, CA, USA, 1995. 
Weick, K. E. (2001). Making Sense of the Organization. Oxford: Blackwell

Weick, K. E. (2002). Puzzles in Organizational Learning: An Exercise in Disciplined Imagination. British Journal of Management, 13, 7-15.

Weick, K. E., Sutcliffe, K. M., \& Obstfeld, D. (2005). Organizing and the Process of Sensemaking. Organization Science, 16, 409-421.

Wheeler, A. R., Richey, R.G., Tokkman, M., \& Sablynski, C. J., (2006). Retaining Employees for Service Competency: The Role of Corporate Brand Identity. Journal of Brand Management, 14 (1), 96-113

Whetten, D. A. (2006). "Albert and Whetten Revisited: Strengthening the Concept of Organizational Identity". Journal of Management Inquiry. 15 (3), 219-234

Whetten, D.A., \& Mackey, A. (2002). A Social Actor Conception of Organizational Identity and its Implications for the Study of Organizational Reputation. Business \& Society, 41, 393-414.

Wickham, P.A. (2001). Strategic Entrepreneurship: A Decision-Making Approach to New Venture Creation and Management. $2^{\text {nd }}$ Edn, London: Pearson Education.

Wieseke, J., Ahearne, M., Lam, S.K., \& van Dick, R. (2009). "The Role of Leaders in Internal Marketing", Journal of Marketing, 73(2), 23-45

Wieseke, J., Ullrich, J., Christ, O., \& Van Dick, R., (2007). Organizational Identification as a Determinant of Customer Orientation in Service Organizations. Marketing. Letters. 18(4), 265-278.

Wilkes, L., \& Halcomb, E. (2013). The Challenges of Being an Insider in Storytelling Research. Nurse Researcher, 21(1), 8-13

Willig, C. (2001). Introducing Qualitative Research in Psychology: Adventures in Theory and Method. Buckingham, Philadelphia: Open University Press 
Wisker, G. (2008). The Postgraduate Research Handbook. Basingstoke, New York: Palgrave Macmillan.

Wood, S.J., Stride, C.B., Wall, T.D., \& Clegg, C.W. (2004). Revisiting The Use And Effectiveness of Modern Management Practices. Human Factors and Ergonomics in Manufacturing, 14, 415-432.

Xenikou, A. (2014). The Cognitive and Affective Components of Organizational Identification: The Role of Perceived Support Values and Charismatic Leadership. Applied Psychology: An International Review, 63(4), 567-588

Yamnill, S., \& McLean, G. N. (2001). Theories Supporting Transfer of Training. Human Resource Development Quarterly, 12(2), 195-208

Yardley, L. (2000). Dilemmas in Qualitative Health Research. Psychology \& Health, 15(2), 215-228. 
ORGANIZATIONAL IDENTIFICATION:

EXPLORING THE USE OF TRAINING AS AN EMPLOYEE IDENTIFICATION MARKER IN SMES 


\section{Participant Information Sheet}

Dear Respondent,

My name is Adetola Adekunle. I am currently a doctoral student at the business school, University of Gloucestershire and working on the thesis to 'Explore the use of training as an employee identification marker within SMEs'.

My thesis hopes to interpret the experiences of 15 SME employees to which I hope you agree to be one regarding their employee organizational identification process and the influence training undertaken within the organization context may have on this process.

I have selected you because you are officially an adult working within a small business organizational setting within the UK economy.

\section{What will I have to do if I take part?}

If you agree to take part, I will ask you to answer some questions. There aren't any right or wrong answers - I just want to hear about your opinions and for you to share your experiences with me. The discussion should take about $1 \mathrm{hr}$ at the most. Please note that some of the questions will relate to your personal opinions and experiences.

\section{Do I have to take part?}

No, taking part is voluntary. If you do not want to take part or choose to stop while the discussion is in progress, you can choose to do this at will and no reason is required. Should you also wish your data not eventually included in the study, you have 14 calendar days from the day of the interview session to let me know about this.

3. If I agree to take part what happens to what I say?

All the information audio-recorded, relayed or given will remain confidential and used for the purposes of this study only. The data will be collected, used, stored and discarded in strict adherence to the University of Gloucestershire research ethics handbook and code of conduct which also significantly mirrors the research governance and organization stipulation in the UK Researcher Development Framework (2010). Your information will also be completely anonymised within my thesis and thus, protect your identity.

\section{What do I do now?}

Think about the information on this sheet, and ask me if you are not sure about anything. If you agree to take part, please sign the consent form. The consent form will not be used to identify you. It will be filed separately from all other information. If, after the discussion, you want any more information about the study please contact:

Dr Philippa Ward
OR

\author{
Dr Robin Bown
}




\title{
Participant Consent Form
}

\section{Research Title}

Organizational identification:

Exploring the use of training as an employee identification marker in SMEs

\author{
Name and contact details of researcher \\ Adetola Adekunle \\ xxxxxxx@gmail.com
}

Please initial box

where applicable

1. I confirm that I have read and understand the information sheet for the above study and have had the opportunity to ask questions.

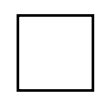

2. I understand that my participation is voluntary and that I am free to withdraw at any time, without giving reason.

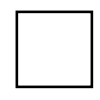

3. I agree to take part in the above study.

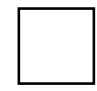

4. I agree to the interview/discussion being audio recorded

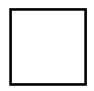

5. I agree to the interview/discussion being video recorded

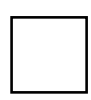

6. I agree to the use of anonymised quotes in publications

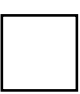

Name of Participant

Date

Signature

Name of Researcher

Date

Signature 


\section{Approved Interview Question Template}

\section{Instruction: Interviewee}

This interview is categorised into 7 main sections (excluding the biodata section), each structured to address the research objectives for the study.

\section{Demographics:}

I. Age (in years):

II. Gender:

III. Name of SME(Optional):

IV. length of stay in SME (in years):

V. Position within the organization:

Theme 1: -What is the perceived understanding of the Ol within employees of SMEs?

1) Can you briefly talk me through what you do at work in a typical day?

2) How would you describe your level of satisfaction with your job

3) What influences this satisfaction?

4) Why do you think that is the case? 
Theme 2: How is Ol characterised by employees of SMEs?

5) Could you explain, in the context of a recent experience at work, whether or not you feel you are relevant within the organization?

6) When you hear people say "I love what I do" or "I love my job" what comes to mind? Why do you think that is?

7) To what extent do you think you are suited to your current organization?

8) Can you elaborate on this please?

Theme 3: What are the perceived markers of employees OI in SMEs?

9) How do you perceive the company's identity or image? And Why?

10) If asked to describe your company, how would you characterise it? 
11) Could you list and elaborate on some of the things that you feel best describe your company?

12) Which of the items you have just talked me through do you feel most aptly describes the company? And Why?

13) Would you say your own identity matches that of the organization?

a. How?

b. And why?

14) Could you describe some of the factors you think help or hinder you to identify with your company's identity?

Theme 4: Is training perceived as an employee OI marker within SMEs?

15) Could you highlight examples of organizational-initiated activities that have been significantly helpful in performing your day-to-day job roles at work?

16) Would you say training activities within the company is one of such significant activities And why? 
Theme 5: If Training is an OI marker, what degree of influence does it wield in the employee Ol process?

17) Can you list the forms or types of training activities offered within your company?

18) How are these training activities most often deployed at work?

19) Could you describe any occasion when training at work has affected your perception of the company or your feelings of belonging to the company?

Theme 6: How is training's degree of influence as an Ol marker characterised by employees within SMEs?

20) Do you think the way you feel about the company's training activities affects your performance in any way?

21) If you were part of management team, would you change some of the training programmes deployed to employees of the company?
a. How?
b. And Why? 
Theme 7: Can employee Ol process be influenced by customising training interventions within the organization?

22) How often do you undergo training at work?

23) Have you undergone any form of training since employed by your company?

24) Thinking about the various in-company training programmes you have undergone, how would you describe their efficacy?

25) Would you consider some training formats or methods more suitable and relevant than others? And why? 